Escuela Técnica Superior de Ingenieros de Telecomunicación

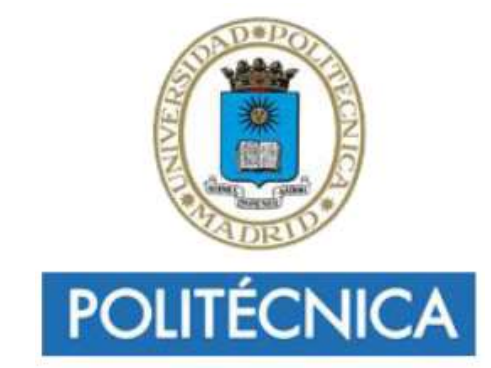

TESIS DOCTORAL

\title{
CONTRIBUCIÓN A LA EJECUCIÓN DE SERVICIOS DISTRIBUIDOS TOLERANTES \\ AL RETARDO, SOBRE REDES DE SENSORES Y ACTUADORES
}

\author{
Autor \\ Edwin Juvenal Cedeño Herrera \\ MSc, Tecnologías de la Información \\ Directores \\ Tomás Robles Valladares \\ Ramón Pablo Alcarria Garrido \\ Departamento de Ingeniería de Sistemas Telemáticos (DIT-UPM), \\ Universidad Politécnica de Madrid (UPM) \\ Departamento de Ingeniería Topográfica y Cartografía (UPM), \\ Universidad Politécnica de Madrid (UPM) \\ Mayo 2017
}





\section{(6.) \\ POLITÉCNICA}

Título de la Tesis: CONTRIBUCIÓN A LA EJECUCIÓN DE SERVICIOS DISTRIBUIDOS TOLERANTES AL RETARDO, SOBRE REDES DE SENSORES Y ACTUADORES

Autor: $\quad$ Edwin Juvenal Cedeño Herrera

Directores: Tomás Enrique Robles Valladares,

Ramón Pablo Alcarria Garrido

Departamento de Ingeniería de Sistemas Telemáticos

Departamento de Ingeniería Topográfica y Cartografía

Tribunal nombrado por el Excmo. y Magfco. Sr. Rector de la Universidad Politécnica de Madrid, el día de Mayo de 2017

Presidente:

Vocal:

Vocal:

Vocal:

Secretario:

Suplente:

Suplente:

Realizado el acto de defensa y lectura de Tesis el día de Mayo de 2017 en la E.T.S. de Ingenieros de Telecomunicación de Madrid.

\section{Calificación:}


A mi amada esposa Gloris Mitzel Batista de Cedeño, a mis adoradas hijas Gloris Denisse Cedeño Batista y Gloris Estefanía Cedeño Batista. Gracias por ser la luz de mi vida. 


\section{Agradecimientos}

Tras un largo camino lleno de alegrías, decepciones, sorpresas y esfuerzo, presento este proyecto de tesis. Aunque la finalización del doctorado ha sido la principal prioridad es estos cinco años, no siempre ha sido posible dedicarle el tiempo suficiente. Aun así estoy muy satisfecho con el trabajo realizado y quiero expresar mi sincera gratitud a las personas sin las cuales la finalización de este trabajo no habría sido posible.

En primera instancia, quiero agradecer a Tomás Robles Valladares, por ser siempre una luz en los tiempos grises de esta larga travesía. Ha sido más que un director de tesis, ha sido un amigo en las buenas y en las malas, siempre con buenos consejos y palabras llenas de ánimo. Con su sabiduría y experiencia me ayudó a conseguir uno de mis más grandes objetivos, el doctorado.

De un modo especial, también quiero agradecer a Ramón Pablo Alcarria Garrido, por ser más que un amigo, un hermano. Ha constituido un soporte permanente, en el plano personal y académico. Siempre con consejos llenos de ánimos, que han permitido seguir luchando para alcanzar la meta. Me ha acompañado muy de cerca en todas las fases de esta tesis, transmitiéndome todo su conocimiento y experiencia. Estaré siempre agradecido por toda su dedicación y esmero.

Agradecer también a los miembros de mi grupo de investigación GISAI, del Departamento de Ingeniería de Sistemas Telemáticos, por compartir inquietudes, risas, ideas y trabajar juntos en la misma dirección. Un especial agradecimiento a Borja, Augusto, Sergio, Héctor, Diego Martín, Álvaro y a Diego Sánchez de Rivera, sin pasar por alto al personal de la ETSIT, por todas las gestiones que me ha ayudado a realizar, siempre con paciencia y amabilidad.

Finalmente dedico esta tesis a mi familia. A mis padres Rosa Edilma Herrera de Martínez y Gil Juvenal Cedeño Terreros, por su soporte incondicional, ayuda y sacrificio durante toda mi vida, especialmente la de estudiante, y a mis hermanos y hermanas, por su consejo y buena compañía. 


\section{Palabras Claves}

Sensores, actuadores, redes tolerantes a fallos, retardos de comunicación, delegación de ejecución de servicios, distribución de servicios, ciclo de vida de servicio, comunicación de máquina a máquina, redes inalámbricas de sensores y actuadores. 


\section{Resumen}

En este trabajo de tesis se proponen soluciones de movilidad para la ejecución de servicios sobre redes WSAN que se encuentran aisladas. Para ello se emplean técnicas que permiten un intercambio de información de forma discontinuada, a través de las características de itinerancia de las Delay Torelant Networks (DTN).

El trabajo de tesis está motivado por la necesidad de disponer de modelos específicos de servicios que contemplen los requerimientos identificados en el dominio de las WSAN desplegadas en zonas aisladas. El desarrollo de una plataforma integrada, que permita ofrecer un framework de gestión de servicios basados en las capacidades proporcionadas por las WSAN, mediante una interfaz basada en tecnologías cloud, representa un aporte significativo para la investigación, ciencia, industria, agricultura y la comunidad de usuarios de WSAN en general.

Para resolver las limitaciones de la ejecución de servicios en ambientes de redes de sensores distribuidas se propone una arquitectura de referencia para la ejecución distribuida de servicios y un modelo de servicio tolerante, definido especialmente para que sea resistente ante retardos.

Para conseguir una distribución y ejecución eficiente de servicios se propone y se evalúa un modelo de ejecución de servicios aportando contribuciones en los procesos de resolución dinámica de componentes, descubrimiento de dispositivos, matchmaking, delegación y ejecución eficiente. 


\section{Abstract}

This thesis proposes mobility solutions for the execution of services on isolated WSAN networks. To do this, techniques are used that allow an information exchange in a discontinuous way, through the roaming characteristics of Delay Torelant Networks (DTN).

This work is motivated by the need to have specific models of services that contemplate the requirements identified in the domain of WSAN deployed in isolated areas. The development of an integrated platform to provide a service management framework based on the capabilities provided by WSAN through a cloud-based interface represents a significant contribution to research, science, industry, agriculture and the user community of WSAN in general.

To solve the limitations of service execution in distributed sensor network environments, a reference architecture for the distributed execution of services is proposed and a tolerant service model, specially defined to be resistant to delays.

In order to achieve an efficient distribution and execution of services, a service execution model is proposed and evaluated, contributing to the processes of dynamic component resolution, device discovery, matchmaking, delegation and efficient execution. 


\section{Contenido}

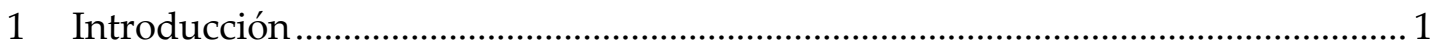

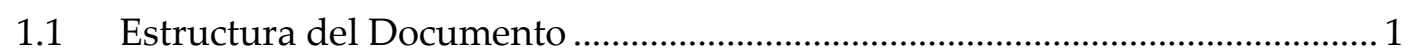

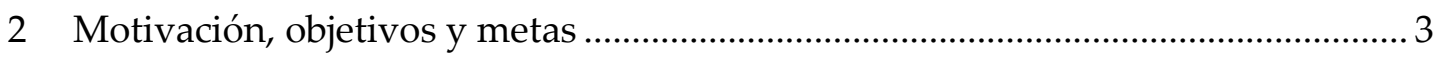

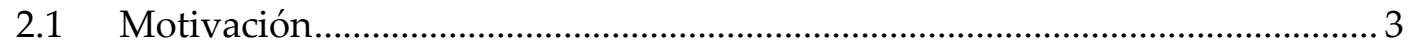

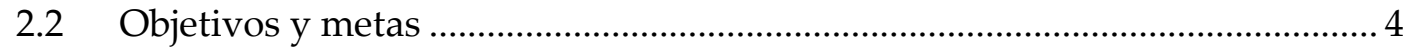

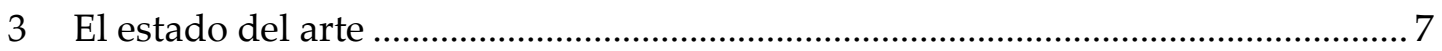

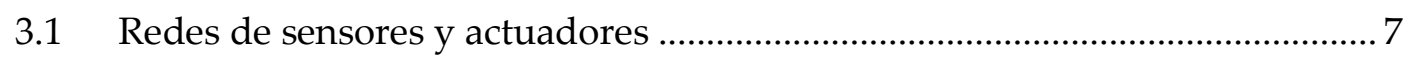

3.2 DTN: Hacia una solución para entornos con comunicaciones limitadas ..... 19

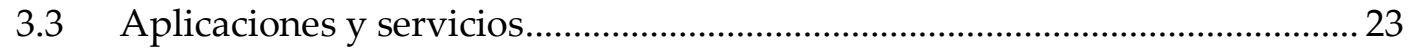

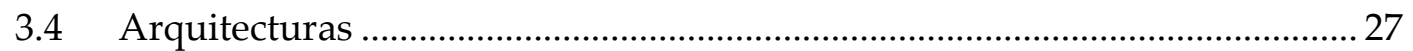

4 Planteamiento de la arquitectura general del sistema .......................................... 33

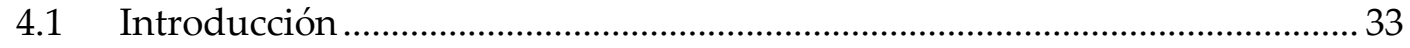

4.2 Descripción de la arquitectura propuesta............................................................ 34

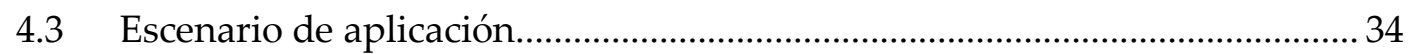

4.4 Requisitos para una arquitectura de servicios sobre redes de sensores ....... 35

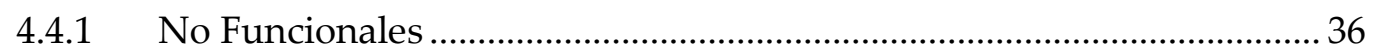

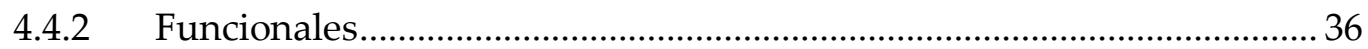

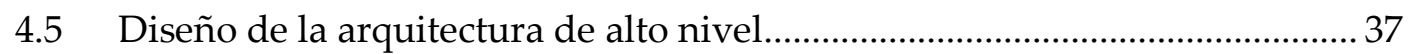

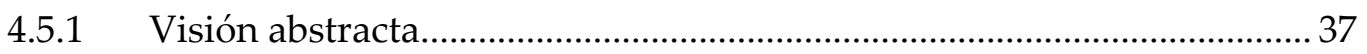

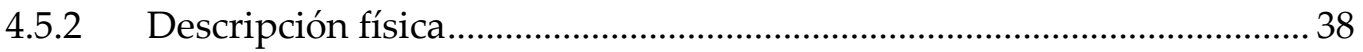

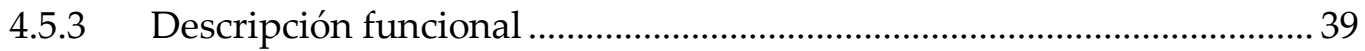

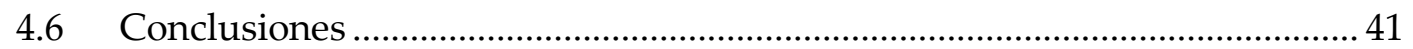

5 Modelo de servicio y gestión del ciclo de vida .................................................... 43

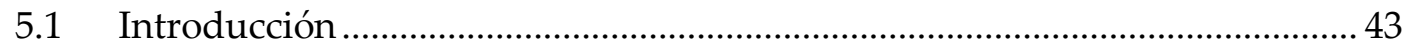

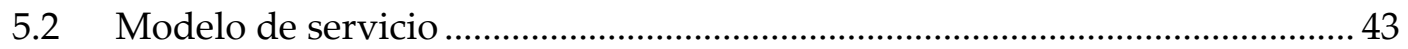

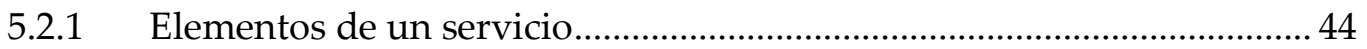

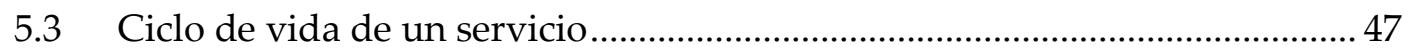

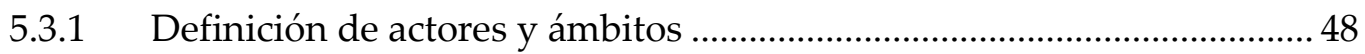

5.3.2 Ciclo de vida de servicios tolerantes ....................................................... 48

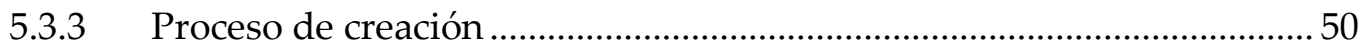

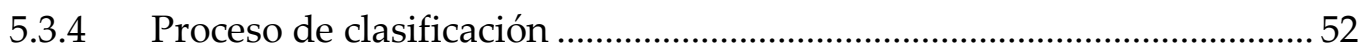




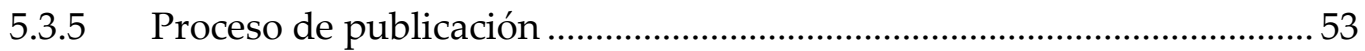

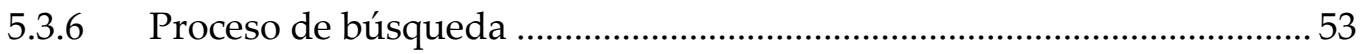

5.3.7 Proceso de adaptación ............................................................................... 54

5.3.8 Proceso de ejecución ...................................................................................... 56

5.3.9 Proceso de verificación ............................................................................... 57

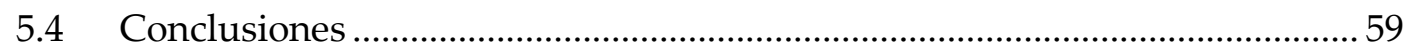

6 Provisión dinámica para la ejecución de servicios ................................................... 61

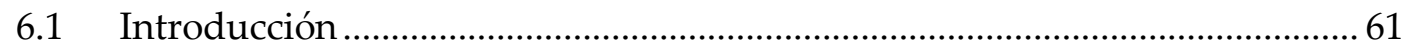

6.2 Modelo de Ejecución de Servicios ....................................................................... 61

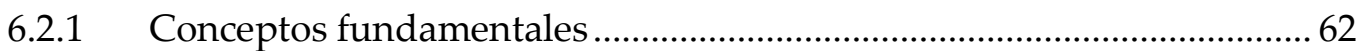

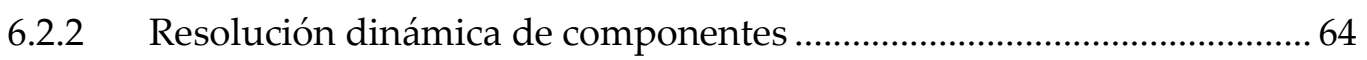

6.2.3 Reconocimiento oportunista de recursos .................................................... 66

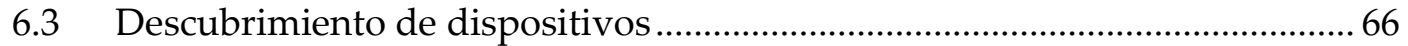

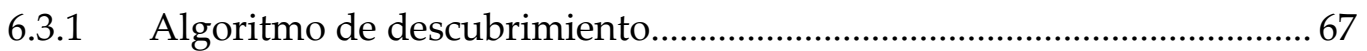

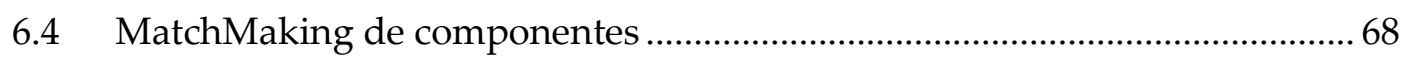

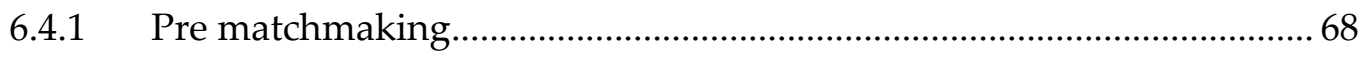

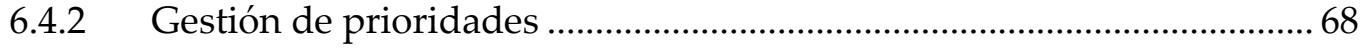

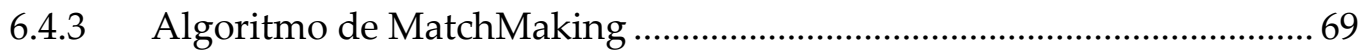

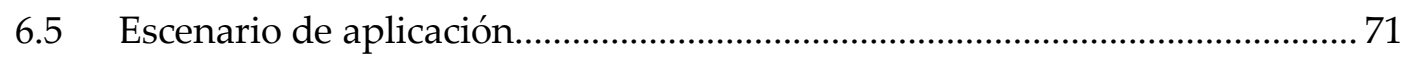

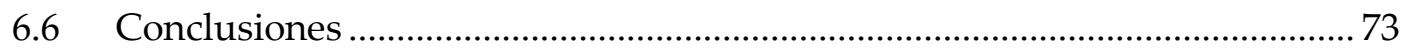

$7 \quad$ Estrategia de delegación de servicios distribuidos................................................... 75

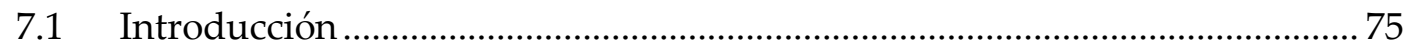

7.2 Estructura general del proceso de delegación.................................................. 75

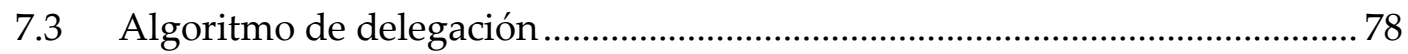

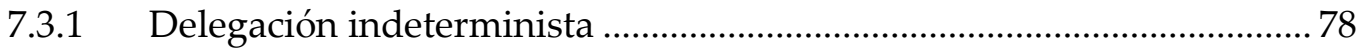

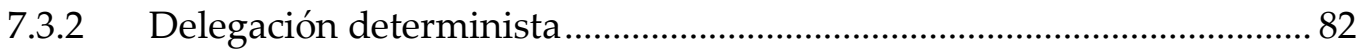

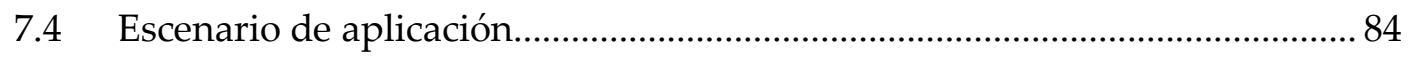

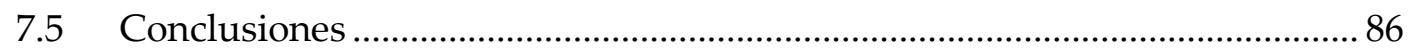

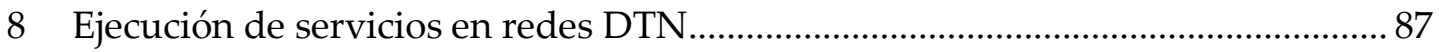

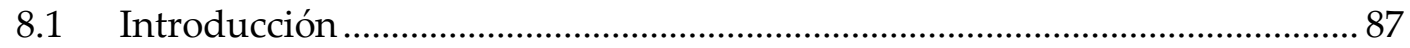

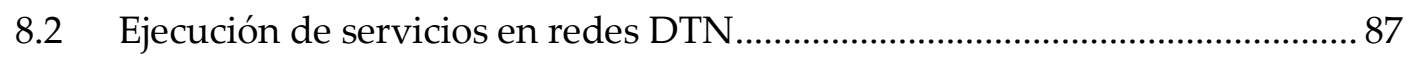

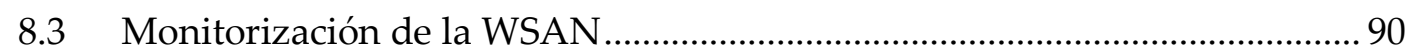

8.4 Ejecución de servicios a través de gestión individual de cluster head ..........91 
8.5 Ejecución de servicios a través de gestión de grupos.....................................97

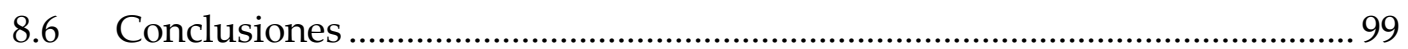

9 Validación de escenarios, simulación y resultados ................................................ 101

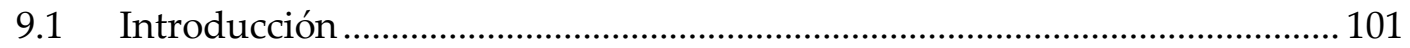

9.2 Definición de escenarios de aplicación............................................................... 102

9.2.1 Escenarios y Casos de Uso ........................................................................ 102

9.2.2 Identificación de requisitos .................................................................. 112

9.3 Diseño y desarrollo de la plataforma de simulación ....................................... 113

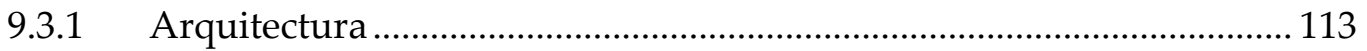

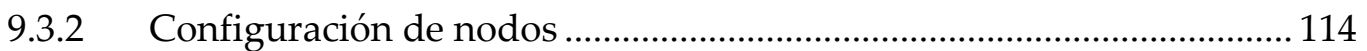

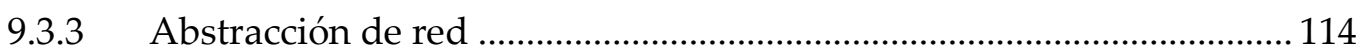

9.3.4 Despliegue de la herramienta de simulación ........................................... 115

9.4 Experimentos y evaluación ........................................................................... 117

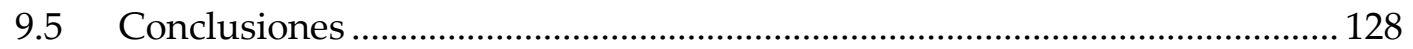

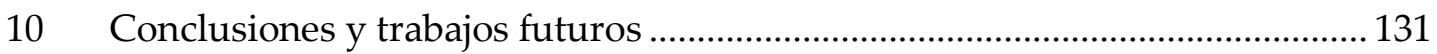

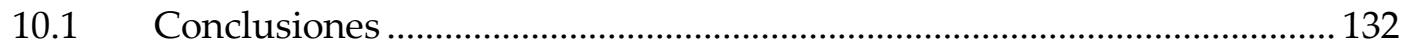

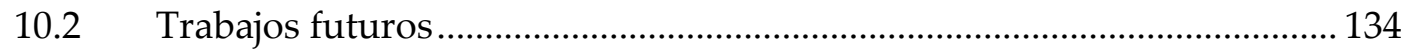

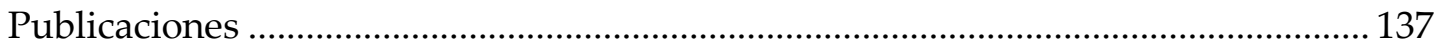

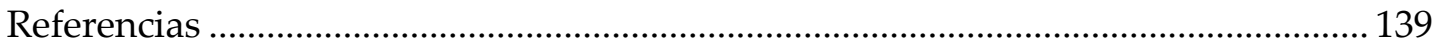




\section{Lista de figuras}

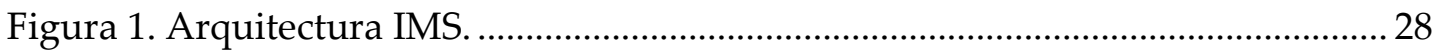

Figura 2. Arquitectura de alto nivel para M2M............................................................ 29

Figura 3. Arquitectura Machine Type Communication. .............................................. 30

Figura 4. Escenario de motivación.................................................................................... 35

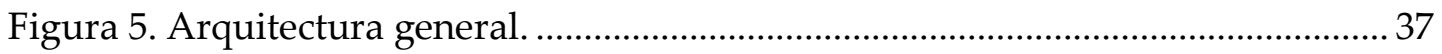

Figura 6. Plano físico de la arquitectura..................................................................... 38

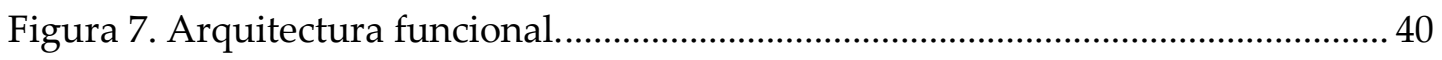

Figura 8. Modelo UML de servicios tolerantes. ................................................................ 45

Figura 9. Procesos del ciclo de vida de un servicio tolerante. ........................................... 49

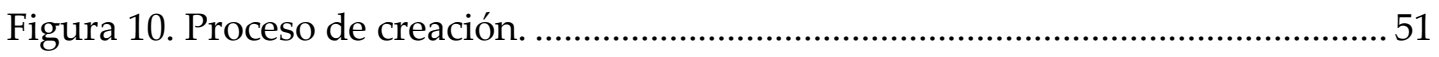

Figura 11. Proceso de clasificación.............................................................................. 52

Figura 12. Proceso de publicación. ................................................................................. 53

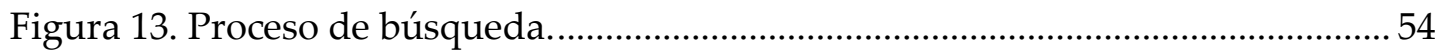

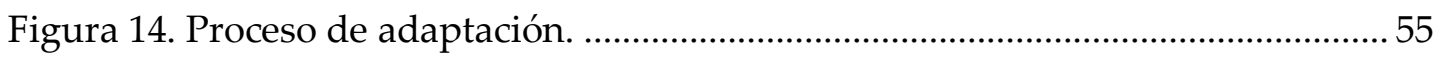

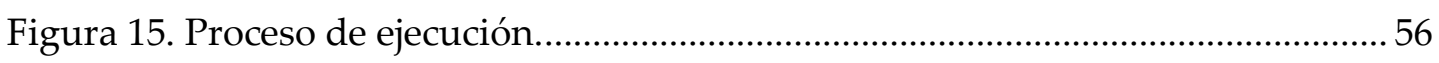

Figura 16. Proceso de verificación. ……….................................................................... 58

Figura 17. Visión global del modelo................................................................................. 64

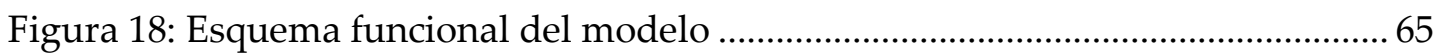

Figura 19: Resolución de componentes y descubrimiento de dispositivos ................. 72

Figura 20: Esquema general del algoritmo de delegación.............................................. 76

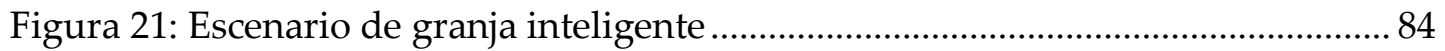

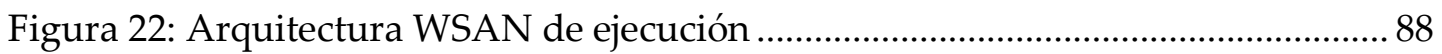

Figura 23: Gestión de servicios en redes DTN ……….................................................. 89

Figura 24: Arquitectura funcional del escenario de aplicación ...................................... 90

Figura 25: Clasificación de servicios dependiendo de los requisitos geográficos...... 92

Figura 26: Diagramas de secuencia de ejecución para el caso A .................................. 93

Figura 27: Diagramas de secuencia de ejecución para el caso B ................................... 93

Figura 28: Diagramas de secuencia de ejecución para el caso C ................................. 94

Figura 29: Diagramas de secuencia de ejecución para el caso D ................................. 94

Figura 30: Diagramas de secuencia de ejecución para el caso E.................................... 95 
Figura 31. Representación gráfica del tiempo de ejecución en gestión de cluster head individual

Figura 32: Diagrama de secuencia de una ejecución de servicios a través de gestión de grupos. 98

Figura 33. Representación gráfica del tiempo de ejecución en gestión de cluster head grupal 99

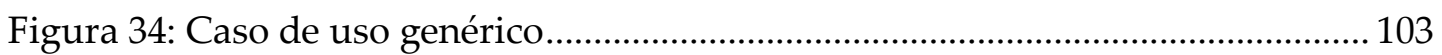

Figura 35: Caso de uso - Generación de capacidades.................................................. 104

Figura 36: Caso de uso - Resolución de componentes ...................................................... 106

Figura 37: Delegación caso básico............................................................................. 108

Figura 38: Caso de uso - Delegación de servicio........................................................... 109

Figura 39: Caso de uso: Ejecución de servicios de control ......................................... 110

Figura 40: Arquitectura de herramienta de simulación............................................... 114

Figura 41: Interfaz gráfica de herramienta de simulación............................................ 115

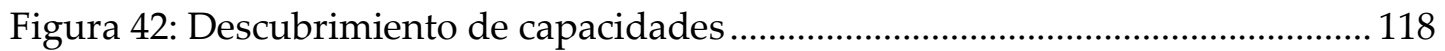

Figura 43: Tiempo de procesamiento de capacidades .................................................. 119

Figura 44: Escalabilidad del algoritmo de descubrimiento ......................................... 120

Figura 45: Evaluación del MatchMaking ………............................................................ 121

Figura 46: Probabilidad de ejecución según cantidad de contactos............................ 122

Figura 47: Probabilidad de fallo en la ejecución según tiempo entre contactos....... 123

Figura 48: Probabilidad de ejecución exitosa .............................................................. 124

Figura 49: La distribución estadística de los resultados de la ejecucíon .................... 125

Figura 50: Tiempo de ejecución resultante del primer experimento ........................... 126

Figura 51: Tiempo de ejecución resultante del segundo experimento ....................... 127

Figura 52: Comparativa de porcentaje de ejecuciones satisfactorias .......................... 128 


\section{Lista de tablas y algoritmos}

\section{Tablas}

Tabla 1: Diferencias entre protocolos de alto nivel: MQTT, MQTT-SN y CoAP ....... 16

Tabla 2: Descripción de los elementos de SPEM............................................................. 47

\section{Algoritmos}

Algoritmo 1: Descubrimiento de dispositivos 67

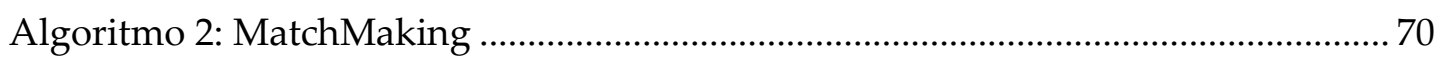

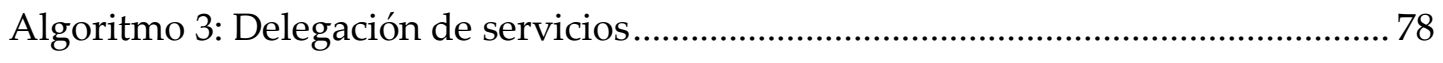

Algoritmo 4: Delegar indeterminista............................................................................ 79

Algoritmo 5: Round trip time service........................................................................... 80

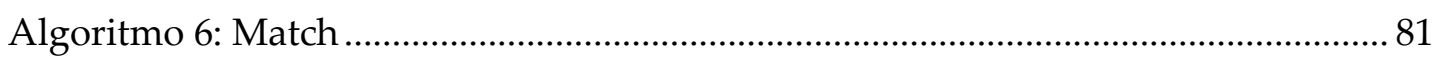

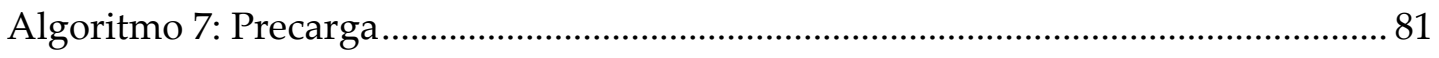

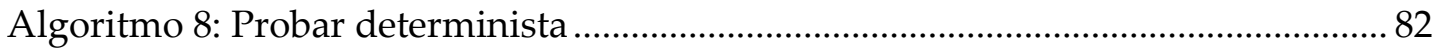

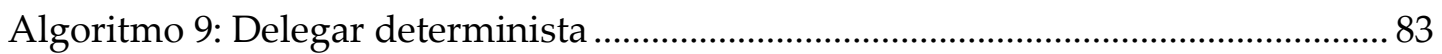




\section{Acrónimos}

Acrónimos en italic corresponden a conceptos definidos en este trabajo de tesis.

\begin{tabular}{|c|c|}
\hline 3GPP & 3rd Generation Partnership Project \\
\hline API & Application programming interface \\
\hline$B C$ & Broker de Comunicaciones \\
\hline$B S$ & Broker de Servicios \\
\hline $\mathrm{BP}$ & Bundle Protocol \\
\hline BPEL & Business Process Execution Language \\
\hline BPMN & Business Process Model and Notation \\
\hline CoAP & Constrained Application Protocol \\
\hline $\mathrm{CN}$ & Core Network \\
\hline DT-WSN & Delay Tolerant Wireless Sensor Networks \\
\hline DTN & Delay Torelant Networks \\
\hline DHT & Distributed Hash Table \\
\hline XML & Extensible Markup Language \\
\hline FC & Factor de Confianza del Servicio \\
\hline FSM & Finite-State Machine \\
\hline GPRS & General Packet Radio Service \\
\hline GSM & Global System for Mobile Communications \\
\hline GUI & Graphical user interface \\
\hline HTTP & Hypertext Transfer Protocol \\
\hline ISM & Industrial, Scientific and Medical \\
\hline $\mathrm{ICN}$ & Information Centric Network \\
\hline IT & Information technology \\
\hline ICNRG & Information-Centric Networking Research Group \\
\hline IETF & Internet Engineering Task Force \\
\hline IoT & Internet of Things \\
\hline IPv6 & Internet Protocol V6 \\
\hline IMS & IP Multimedia Subsystem \\
\hline 6LowPAN & IPv6 over Low power Wireless Personal Area Networks \\
\hline LTP & Licklider Transmission Protocol \\
\hline LXC & Linux Containers \\
\hline LTE & Long Term Evolution \\
\hline M2M & Machine to machine \\
\hline MTC & Machine Type Communication \\
\hline MM & MatchMaking \\
\hline MAC & Media Access Control \\
\hline MQTT & Message Queue Telemetry Transport \\
\hline MQTT & Message Queue Telemetry Transport \\
\hline MF & Mobile Ferries \\
\hline MULE & Mobile Ubiquitous LAN Extensions \\
\hline MQTT-SN & MQTT For Sensor Networks \\
\hline MIMO & Multiple Input Multiple Output \\
\hline NS-3 & Network simulator version 3 \\
\hline NGNs & Next Generation Networks \\
\hline OMG & Object Management Group \\
\hline
\end{tabular}




\begin{tabular}{|c|c|}
\hline OOP & Object Oriented Programming \\
\hline OMA & Open Mobile Alliance \\
\hline OSA & Open Standard Alliance \\
\hline OSI & Open System Interconnection \\
\hline OASIS & $\begin{array}{l}\text { Organization for the Advancement of Structured Information } \\
\text { Standards }\end{array}$ \\
\hline $\mathrm{P} 2 \mathrm{P}$ & Peer-to-peer \\
\hline PDA & Personal Digital Assistant \\
\hline PSR & Proveedor de Servicios de Red \\
\hline$P A$ & Proxy de Acceso \\
\hline $\mathrm{Pub} / \mathrm{Sub}$ & Publish-subscribe \\
\hline QoS & Quality of service \\
\hline QoS & Quality of Service \\
\hline RF & Radio Frequency \\
\hline$R M D$ & Repositorio de Mulas Deterministas \\
\hline RMI & Repositorio de Mulas Indeterministas \\
\hline RTTS & Round Trip Time Service \\
\hline SDL & Service Description Language \\
\hline SOA & Service oriented architecture \\
\hline SoaML & Service oriented architecture Modeling Language \\
\hline SPF & Service Provisioning Framewaork \\
\hline SDS & Servidor de Dispositivos Suscriptores \\
\hline SIP & Session Initiation Protocol \\
\hline SOAP & Simple Object Access Protocol \\
\hline SID & Sistema de Información de Dispositivos \\
\hline SPEM & Software Process Engineering Meta-Model \\
\hline SPL & Software Product Line \\
\hline SCADA & Supervisory Control And Data Acquisition \\
\hline TISPAN & $\begin{array}{l}\text { Telecommunications and Internet Converged Services and Protocols } \\
\text { for Advanced Networking }\end{array}$ \\
\hline TINA & Telecommunications Information Networking Architecture \\
\hline TMN & Telecommunications Management Network \\
\hline TCP & Transmission Control Protocol \\
\hline UML & Unified Modeling Language \\
\hline UMTS & Universal Mobile Telecommunications System \\
\hline UUID & Universally unique identifier \\
\hline UAVs & Unmanned Aerial Vehicles \\
\hline UDP & User Datagram Protocol \\
\hline VoIP & Voice Over IP \\
\hline WSDL & Web Service Definition Language \\
\hline Wi-Fi & Wireless Fidelity (ver WLAN) \\
\hline WLAN & Wireless Local Area Network \\
\hline WSAN & Wireless Sensor and Actuator Network \\
\hline WSN & Wireless Sensor Networks \\
\hline WWW & World Wide Web \\
\hline WiMAX & Worldwide Interoperability for Microwave Access \\
\hline
\end{tabular}




\section{Introducción}

Las redes de sensores inalámbricas o Wireless Sensor Networks (WSN) han supuesto un avance significativo en los entornos de comunicaciones inalámbricos y su convergencia con el fenómeno denominado Internet de las Cosas. Entre los elementos y paradigmas estudiados en el campo de los WSN están la propuesta de mecanimsos de comunicación para interconectar zonas aisladas. En este caso de estudio, las redes pertenecientes a una localización geográfica se denominan Wireless Sensor Area Networks (WSAN).

Diversos estudios han puesto de manifiesto la dificultad de lograr conectividad en este tipo de redes. Más aún, considerando que la red de comunicaciones es una infraestructura de soporte a la gestión del ciclo de vida de servicios en movilidad, las necesidades y los requerimientos de este tipo de redes deben observarse desde un nivel de abstracción aún mayor y se deben proponer otros mecanismos para la ejecución de servicios ubicuos.

En este trabajo de tesis se proponen soluciones de movilidad para la ejecución de servicios sobre redes WSAN que se encuentran aisladas. Par ello se emplean técnicas que permiten un intercambio de información de forma discontinuada, a través e las características de itinerancia de las Delay Torelant Networks (DTN).

El trabajo de tesis está motivado por la necesidad de disponer de modelos específicos de servicios que contemplen los requerimientos identificados en el dominio de las WSAN desplegadas en zonas aisladas. El desarrollo una plataforma integrada, que permita ofrecer un framework de gestión de servicios basados en las capacidades proporcionadas por las WSAN, mediante una interfaz basada en tecnologías cloud, representa un aporte significativo para la investigación, ciencia, industria, agricultura y la comunidad de usuarios de WSAN en general.

\subsection{Estructura del Documento}

Esta disertación está estructurada de la siguiente forma: 
Sección 2, Motivación, objetivos y metas, describe la motivación de esta tesis, las metas a lograr y los objetivos particulares para lograr las metas.

Sección 3, Estado del Arte, analiza los trabajos relacionados con respecto a las contribuciones propuestas y cómo estas contribuciones cumplen con los objetivos establecidos en la Sección 2.

Sección 4, .Planteamiento de la arquitectura, propone una nueva arquitectura para el soporte de servicios tolerantes al retardo, sobre redes de sensores y actuadores. Presenta una descripción completa de la arquitectura y los escenarios de aplicación. Contiene una identificación de los requisitos para una arquitectura de servicios sobre WSAN aisladas. El diseño de la arquitectura de alto nivel, también es presentado en esta sección.

Sección 5, .Modelo de servicio y gestión del ciclo de vida, propone un modelo de servicio tolerante al retardo, y los procesos o etapas de su ciclo vida. Describe los distintos itinerarios que puede serguir el servicio desde su creación hasta su ejecución.

Sección 6, Provisión dinámica para la ejecución de servicios, presenta los mecanismos que permiten la contínua ejecución de los servicios tolerantes al retardo. Se definen los algoritmos necesarios para el descubrimiento de dispositivos y el maching de componentes del servicio, así como también un escenario de aplicación.

Sección 7, Estrategia de delegación de servicio, describe la característica de delegación, esta es fundamental en la arquitectura propuesta en la sección 4. Propone un algoritmo de delegación y se describe en detalle el escenario de aplicación.

Sección 8, Ejecución de servicios en redes DTN, se propone una estratégia de ejecución de servicios sobre redes de sensores y actuadores, basada la gestión individual y grupal de servicios a bajo nivel.

Sección 9, Validación de escenarios, simulación y resultados, se definen los escenarios de aplicación y sus casos de uso. Se identifican las características necesarias en la plataforma de validación. Se presenta el diseño y desarrollo de una plataforma de simulación según los requisitos. Finalmente, se presentan los resultados de los experimientos y su análisis.

Finalmente, Sección 10, Conclusiones y Trabajos Futuros, resumimos los logros de las contribuciones de este trabajo de tesis y describimos las actuales líneas de investigación que prosiguen el trabajo de esta disertación. 


\section{Motivación, objetivos y metas}

En esta sección, describo la motivación de la tesis y las metas a lograr. Estas metas son detalladas en objetivos particulares, los cuales identifico en esta sección, y que son cubiertos por las contribuciones desarrolladas en este trabajo de tesis.

\subsection{Motivación}

Las redes inalámbricas de sensores (WSN) han proliferado en muchos campos, incluyendo ambientes hostiles y zonas aisladas en donde se cuentan con infraestructuras de comunicaciones muy limitadas o inexistentes. Las redes de sensores juegan un papel importante en la recolección de información, y permite resolver muchos problemas, como es el caso de desastres naturales, nucleares y medioambientales entre otros. En el ámbito de las redes inalámbricas de sensores con comunicaciones limitadas, la recuperación, procesamiento y posterior transformación en servicios disponibles para la comunidad científica, sigue sin tener solución, por lo que ésta se considera una necesidad que no ha sido resuelta de forma adecuada, es por esta razón, que es necesario proponer una alternativa eficiente para la creación, despliegue y ejecución de servicios en ambientes de redes de sensores distribuidas.

Las WSN inicialmente fueron diseñadas y creadas con el objetivo de recolección de datos, sin embargo, estas han evolucionado incorporando actuadores, por lo que hoy día las conocemos como redes inalámbricas de sensores y actuadores (WSAN). Por otro lado, el surgimiento y estandarización de nuevos protocolos y tecnologías específicas, han permitido el desarrollo de nuevas aplicaciones que requieren comunicaciones bidireccionales asíncronas con las redes inalámbricas de sensores/actuadores. Sin embargo, existen factores que limitan las funcionalidades que estas pueden ofrecer, estos factores están relacionados con los limitados recursos con que cuentan estos dispositivos sensores/actuadores (baja tasa de comunicación de datos, buffer, procesador, autonomía energética y otros), por lo tanto, es preciso el desarrollo de una plataforma que permita el despliegue de servicios sobre redes inalámbricas de sensores/actuadores, que contemple las limitaciones de los dispositivos participantes en la ejecución de los servicios distribuidos. 
Es fundamental proponer una arquitectura integral que permita superar las limitaciones de comunicación entre las redes de sensores/actuadores desplegadas en zonas remotas, y la cloud, considerando el desafío de la itinerancia en que se llevan a cabo las comunicaciones en este escenario. En este trabajo de tesis se han estudiado varias alternativas propuestas para resolver el problema de las discontinuas y limitadas comunicaciones con redes de sensores/actuadores en zonas aisladas, las cuales son presentadas en la sección 3.2. Sin embargo, la mayoría están enfocadas a resolver el problema desde el punto de vista de las capas bajas del modelo OSI. Adicionalmente, las alternativas propuestas no contemplan el problema de la ejecución de servicios distribuidos en este contexto. En este orden de ideas, existen nuevas tecnologías emergentes, como las "Delay Tolerant Nerwork (DTN)", que pueden ser adaptadas en estos entornos con el objetivo de resolver los problemas de comunicación inherentes, y ofrecer soporte para la gestión de servicios distribuidos.

Teniendo en cuenta lo anteriormente planteado, es indispensable el diseño de un modelo específico de servicios, que contemple los requerimientos identificados en el dominio de las WSAN desplegadas en zonas aisladas. La definición de dichos servicios debe incluir estados especiales del servicio o el ciclo de vida, debido a los limitados recursos con que cuentan los dispositivos y la discontinua participación (desconexión y reconexión con enfoque oportunista) de estos dispositivos en la ejecución de los servicios distribuidos. La colaboración entre múltiples WSAN en la ejecución de servicios distribuidos, permitirá la resolución del servicio más cerca al origen de los datos, proporcionando mayor eficiencia en la ejecución, comparado con el enfoque de ejecución de servicios centralizados. El desarrollo una plataforma integrada, que permita ofrecer un framework de gestión de servicios basados en las capacidades proporcionadas por las WSAN, mediante una interfaz basada en tecnologías cloud, representa un aporte significativo para la investigación, ciencia, industria, agricultura y la comunidad de usuarios de WSAN en general.

\subsection{Objetivos y metas}

El objetivo principal de esta tesis es el diseño de una solución para la ejecución de servicios distribuidos sobre redes de sensores y actuadores en zonas remotas, utilizando las características de los protocolos de las DTN, de tal forma que se pueda resolver las limitaciones de acceso y comunicación con las distintas entidades, en entornos con infraestructura de comunicaciones limitadas.

Objetivo \#1: Diseño de una de arquitectura de referencia y funcional para la ejecución distribuida de servicios sobre redes inalámbricas de sensores y actuadores con infraestructura de comunicación limitada, desplegadas en zonas aisladas o desatendidas.

Objetivo \#2: Identificar los requisitos funcionales y no funcionales que deben satisfcaer las arquitecturas propuestas en el Objetivo \#1, a fin de permitir una 
ejecuación eficaz de servicios tolerantes a retardo sobre redes de sentrores y actuadores aisladas

Objetivo \#3: Crear un modelo de servicios en el contexto de la arquitectura definida en el Objetivo \#1, que considere los requerimientos de las comunicaciones limitadas entre las redes inalámbricas de sensores/actuadores y una herramienta de soporte para el desarrollo de servicios basada en cloud.

Objetivo \#4: Proponer un modelo de gestión de servicios distribuidos tolerantes a retardo que permita la creación, distribución y ejecuación eficiente de los mismos empleando infraestructuras de WSAN aisladas que incluyan nodos móviles con capacidades de diseminación de mensajes y acceso a la cloud.

Objetivo \#5: Definir el ciclo de vida de los servicios del Objetivo \#3, considerando la ejecución en entornos distribuidos, la movilidad del servicio, tolerancia a largos retardos y las fallas en la infraestructura de comunicación.

Objetivo \#6: Proponer un framework de ejecución de servicios distribuidos tolerantes al retardo a alto nivel, que permita la identificar aquellos serviciso elegibles para ser ejecutados sobre una determinada infraestructura.

Objetivo \#7: Desarrollar una estrategia flexible y eficiente para la delegación y distribución servicios basados en el framework definido en el Objetivo\#6, que considere los requerimientos de las redes inalámbricas de sensores/actuadores desplegadas en ambientes hostiles o remotos.

Objetivo \#8: Formular una estrategia de ejecución de servicios a bajo nivel sobre nodos en redes de sensores y actuadores con comunicaciones limitadas.

Objetivo \#9: Desarrollo de una plataforma de simulación monolítica, liviana, configurable, flexible, altamente escalable y confiable, que proporcione soporte para simulaciones de ambientes distribuidos con comunicaciones limitadas, inalámbricas, disruptivas, y que permita la experimentación de las aplicaciones, prototipos y pruebas de conceptos, que validan las contribuciones en los distintos escenarios. 


\section{El estado del arte}

Esta sección se analiza los trabajos relacionados a las contribuciones propuestas, mientras enlazamos las más importantes características de estas tecnologías, proyectos, modelos o algoritmos, con los objetivos propuestos en la Sección 2.

\subsection{Redes de sensores y actuadores}

En años recientes se ha notado un creciente interés en el diseño y desarrollo de Wireless Sensor and Actuator Network (WSAN). Los avances en tecnología micro electrónica, comunicaciones inalámbricas y los precios, han permitido el despliegue de WSAN de bajo costo, y a gran escala en muchos ámbitos (salud, domótica, militar, gestión de desechos tóxicos y otros). Las WSAN permiten el monitoreo y control del ambiente físico en localizaciones remotas con mayor precisión. Las múltiples aplicaciones de las tecnologías de WSAN ha atraído la atención de investigadores en la academia y la industria (Ting-hui, Sang, \& Yi-min, 2010).

Las WSAN son una nueva clase de redes heterogéneas, y comprenden un grupo de nodos sensores y actuadores que se conectan de manera inalámbrica. Son consideradas una extensión de las Wireless Sensor Network (WSNs), con la característica de que permiten a sus nodos actuadores, tomar decisiones autónomas y ejecutar acciones en función de las lecturas de los nodos sensores (Ruiz-Ibarra \& Villasenor-Gonzalez, 2008). Estas nociones permiten describir de forma concreta las interacciones entre el sistema y su entorno (Abdelhaq \& Houria, 2008). La nueva arquitectura de las WSAN, permite ejecutar no solo operaciones de lectura, sino también operaciones de escritura, lo que plantea nuevos desafíos que deben abordarse (Ramanuja, Zhenyun, \& Raghupathy, 2006). La coexistencia de nodos sensores y actuadores requiere satisfacer nuevos requerimientos, para esto es necesario la implementación de múltiples niveles de coordinación, los cuales están definidos como: Sensor-Sensor, Sensor-Actuador y Actuador-Actuador (Akyildiz \& Kasimoglu, 2004).

Debido a las características particulares de WSAN existen aún muchos temas por resolver. Las fuentes de energía de cada sensor son limitadas, por lo tanto no soportan 
comunicaciones a grandes distancias, el reemplazo de baterías no siempre es factible en este tipo de redes, sobre todo si están desplegadas en zonas remotas o desatendidas (Asim, Mokhtar, H., \& Merabti, M., 2008).

\section{Tecnologías de redes de sensores y actuadores (WSAN)}

Las WSAN están caracterizadas por una alta heterogeneidad, debido a que han sido desarrolladas por múltiples fabricantes, que han ofrecido soluciones propietarias y no propietarias, sin considerar un estándar como denominador común. Este amplio rango de tecnologías ha provocado un retardo en el despliegue de nuevos desarrollos, y la integración de las redes de sensores existentes. Sin embargo, la tendencia actual es alejarse de las soluciones propietarias y cerradas, para enfocarse en la integración de nuevos y emergentes estándares basados en soluciones IP (Mainetti, Patrono, \& Vilei, 2011).

Una revisión de las principales tecnologías utilizadas por las WSAN, incluye las soluciones que no están basadas en el Protocolo de Internet, el nuevo estándar de 6LoWPAN, el middleware y las tecnologías de alto nivel.

\section{Tecnologías No IP}

ZigBee es una tecnología de redes inalámbricas desarrollada por la ZigBee Alliance para aplicaciones de corto rango y baja tasa de transferencia de datos (ZigBee Alliance, 2010b). El stack del protocolo ZigBee está compuesto de cuatro capas principales: la capa física (PHY), control de acceso al medio (MAC), la capa de red (NWK), y la de aplicación (APL). La capa PHY y MAC de ZigBee está definida por el estándar IEEE 802.15.4, mientras que es resto del stack está definido por la especificación de ZigBee.

La versión inicial del IEEE 802.15.4, en el cual está basado ZigBee, opera en la banda de los $868 \mathrm{MHz}$ (Europa), $915 \mathrm{MHz}$ (Norte América) y $2.4 \mathrm{GHz}$ (en todo el mundo). Las tazas de datos son $20 \mathrm{~kb} / \mathrm{s}, 40 \mathrm{~kb} / \mathrm{s}$, y $250 \mathrm{~kb} / \mathrm{s}$ respectivamente.

La capa de red de ZigBee soporta específicamente direccionamiento y ruteo para topologías de árbol y malla. El desarrollo de aplicaciones Zigbee se basa en perfiles. Los más importantes perfiles de aplicaciones ZigBee son el ZigBee Home Automation Public Application Profile (ZigBee Alliance, 2010b) y el ZigBee Smart Energy Profile (ZigBee Alliance, 2008). Las principales áreas de aplicación para el perfil de Home Automation son la iluminación, climatización y seguridad. En cambio el perfil de Smart Energy está orientado hacia la respuesta ante la demanda de energía y las aplicaiones de gestión de carga para redes eléctricas. La nueva especificación de ZigBee es RF4CE (ZigBee Alliance, 2010a), que tiene un stack de red simplificado sólo para topologías de estrella, ofreciendo una solución simple para el control remoto de electrónica de consumo.

Z-Wave es una arquitectura de protocolo inalámbrico desarrollado por Zensys y promovida por la Z-Wave Alliance, para la automatización en entornos residenciales y comerciales pequeños. 
El propósito principal de Z-Wave es permitir la transmisión fiable de mensajes cortos desde una unidad de control a uno o más nodos de la red (Zensys A/S, 2007). ZWave está organizado de acuerdo a una arquitectura compuesta por cinco capas principales: PHY, MAC, transferencia, enrutamiento, y de aplicación. La radio Z-Wave opera principalmente en el $900 \mathrm{MHz}(868 \mathrm{MHz}$ en Europa y $908 \mathrm{MHz}$ en los Estados Unidos) y 2,4 GHz. Z-Wave permite taza de transmisión de datos de $9.6 \mathrm{~kb} / \mathrm{s}, 40 \mathrm{~kb} / \mathrm{s} \mathrm{y}$ $200 \mathrm{~kb} / \mathrm{s}$.

Z-Wave define dos tipos de dispositivos: los controladores y los esclavos. Los controladores sondean o envían comandos a los esclavos, los cuales responden a los controladores o ejecutan comandos. La capa de enrutamiento de Z-Wave, realiza el enrutamiento basado en un enfoque de enrutamiento de origen.

INSTEON Alliance promueve una solución llamada INSTEON, desarrollada por SmartLabs y orientada hacia la Home Automation. Una característica distintiva de INSTEON es que define una topología de malla compuesta de enlaces de RF y líneas de alimentación. Los dispositivos pueden ser de RF únicamente o línea de alimentación exclusivamente o soportar ambos tipos de comunicación, ofreciendo un sistema de comunicación redundante, asegurando la transmisión de los datos ante eventuales de fallos. Esta solución opera en una frecuencia central de $904 \mathrm{MHz}$, con una taza de transferencia de $38.4 \mathrm{~Kb} / \mathrm{s}$ (Darbee, 2013).

En INSTEON los dispositivos se comportan como pares, lo que significa que cualquiera de ellos puede desempeñar el papel de emisor, receptor, o repetidor. La comunicación entre los dispositivos que no están dentro del mismo rango se asegura por medio de un enfoque de salto múltiple, que se basa en un esquema de sincronización de ranura de tiempo.

Por su parte WAVENIS, es un stack de protocolo inalámbrico de bajo poder, desarrollado por Coronis System, para aplicaciones de control y vigilancia en algunos entornos. Actualmente es promovido y gestionado por Wavenis Open Standard Alliance (Wavenis-OSA). Este define las funcionalidades de las capas física, enlace y de red. Los servicios de Wavenis, pueden ser accesibles desde las capas superiores, a través de una Application Programming Interface (API).

Wavenis opera principalmente en los $433 \mathrm{MHz}, 868 \mathrm{MHz}$ y $915 \mathrm{MHz}$, que son bandas ISM (Industrial, Scientific and Medical) en Asia, Europa y los Estados Unidos. Algunos productos operan en la banda de los $2.4 \mathrm{GHz}$. Las tazas mínimas y máximas ofrecidas por Wavenis son $4.8 \mathrm{~Kb} / \mathrm{s}$ y $100 \mathrm{~Kb} / \mathrm{s}$, respectivamente, con $19.2 \mathrm{~Kb} / \mathrm{s}$ como valor típico.

\section{Tecnologías basadas en IP}

Los esquemas de direccionamiento en las WSAN no son estándares, cada fabricante utiliza esquemas diferentes, esta diversidad de esquemas es uno de los factores importantes que inciden en la integración entre las WSAN de diferentes marcas, y 
también en la conexión de estas redes a la red Internet, de cara a brindar servicios globales a la comunidad de usuarios.

Las WSAN basadas en sistemas cerrados o propietarios, se comportan como islas de conectividad limitada con el mundo exterior, por lo tanto, es necesaria la implementación de complejos proxies con conocimientos específicos a nivel de capa de aplicación, para exportar los datos de las WSAN hacia otros equipos conectados a Internet. Sin embargo, la tendencia es utilizar IP, para asegurar una conexión nativa entre WSAN y la Internet (Vasseur \& Dunkels, 2010). De esta forma, los smart objects (sensores y actuadores con interfaces de red) pueden estar interconectados basándose en estándares abiertos, en el cual cada dispositivo tienes su propia dirección IP.

Debido al aumento exponencial en el número de dispositivos conectados para el 2020, según los pronósticos: 25 billones-Gartner (“Gartner Symposium/ITxpo," 2014) y 50 billones-Ericsson (“Connected Devices - Ericsson," 2012), el IPv4 no puede ser utilizado por su limitado espacio de direccionamiento. Una mejor opción es utilizar IPv6 debido a su amplio espacio de direccionamiento, con 128 bits para direcciones, además, IPv6 permite autoconfiguración de red y operación sin estado.

La utilización de Six Low Power Wireless Personal Area Networks (6LoWPAN) es ideal para aplicaciones en las que dispositivos embebidos necesitan comunicarse con los servicios basados en Internet, utilizando estándares abiertos, capaces de escalar a través de grandes infraestructuras de red, y con gestión de la movilidad.

Dado el limitado tamaño del paquete y otras restricciones de LoWPAN, una capa de adaptación para compresión de cabeceras, fragmentación y la auto-configuración de direcciones, es necesaria para utilizar IPv6 (Shelby \& Bormann, 2009). El 6LoWPAN IETF Working Group, ya ha definido el formato para la adaptación entre IPv6 y el IEEE 802.15.4.

La arquitectura de 6LoWPAN está desarrollada encima de LoWPAN, que esta conectadas a otras rede IP a través de routers edge. El router edge juega un papel muy importante, ya que enruta el tráfico de entrada y salida de la LoWPAN, controla la compresión de 6LoWPAN, el Neighbor Discovery para el LoWPAN, y si el LoWPAN está conectado a redes $\mathrm{IPv} 4$, el router edge puede controlar la interconectividad IPv4 también. Cada nodo LoWPAN se identifica por una dirección IPv6 única, y es capaz de enviar y recibir paquetes IPv6. La adaptación entre las direcciones IPv6 completas y el formato de LoWPAN es realizado por los routers edge. Esta transformación es transparente, eficiente y sin estado en ambas direcciones. Además, 6LoWPAN no requiere una infraestructura para operar, ya que también puede funcionar en modo adhoc LoWPAN.

\section{Middleware}

Los principales factores que condicionan el desarrollo y despliegue de las WSAN, están relacionados con las restricciones de los dispositivos que las conforman, las tecnologías de comunicación y la heterogeneidad de los entornos en donde operan. 
Por esta razón, el middleware para WSAN debe proporcionar abstracciones, sobre el nivel de complejidad de los mecanismos subyacentes, formando una imagen homogénea y concisa de la red, y de esta forma cambiar el enfoque del programador, de las capas bajas, hacia los temas de alto nivel.

Actualmente en el despliegue de WSAN, es necesario enfocar y conocer el incremento en la demanda de diversas aplicaciones cada vez más exigentes, que se caracterizan por requisitos altamente dinámicos. Esta necesidad está evidenciada por la amplia gama de plataformas actuales, caracterizadas por diferente disponibilidad de recursos, capacidades y el soporte a sensores y actuadores. A continuación, se presenta una revisión de las principales plataformas de middleware que han sido propuestas.

OASIS (Kushwaha, Amundson, Koutsoukos, Neema, \& Sztipanovits, 2007) es un middleware orientado a servicio para WSN, basado en una abstracción de programación centrada en el objeto. Los servicios expresan cada funcionalidad disponible en la WSN, proporcionando interfaces bien especificadas, que definen la interconexión entre unos y otros, y conducen el proceso de desarrollo de aplicaciones, mientras que ellos son implementados en los nodos de la red formando un despliegue heterogéneo. Toda la red está programada con una única aplicación, siendo definida como un objeto con estados específicos (Máquina de estados finitos - FSM) y acciones de red correspondientes, como está indicado por la interconexión de los bloques de servicio. La ejecución se inicia en un nodo basado en los criterios del usuario, teniendo previamente descubiertos por el middleware, los servicios requeridos. Mientras que su transición de estado y la migración a otros nodos, se realizan de acuerdo a un mantenimiento y a un protocolo de migración respectivamente, basada en la evaluación, definida por las condiciones de uso.

RUNES (Costa, Coulson, et al., 2007) es un middleware basado en componentes para WSN, diseñado e implementado específicamente para permitir reconfiguración dinámica de una aplicación o parte de un sistema, en tiempo de ejecución, sin necesidad de interrupción de la operación normal del sistema, y disminuir el consumo de energía y congestión de la red, que imponen métodos tradicionales que emplean mecanismos de configuración a través del reemplazo binario. La definición de los componentes funcionales del sistema WSN, se organiza junto con sus interconexiones, a través de interfaces y recipientes, basados en un modelo de componentes estándar OpenCom (Coulson et al., 2008), que impulsa todo el proceso de composición de software y determina la granularidad de reconfiguración.

REMOWARE (Amir Taherkordi, Loiret, Rouvoy, \& Eliassen, 2013) es un middleware modular para WSN, que provee una abstracción de programación basada en componentes, para el despliegue dinámico de aplicaciones, apoyándose y extendiendo REMORA (Amirhosein Taherkordi et al., 2010), modelo de programación de alto nivel, para el soporte de reconfiguración de componentes funcionales que constituyen una aplicación. REMORA adopta un paradigma de programación basado en componente, y soporta la conexión de componentes orientada a eventos, a través de 
la provisión de una API adecuada, considerando las limitaciones de recursos y la naturaleza orientada a eventos de las WSN. REMOWARE proporciona un API que permite la vinculación dinámica de los componentes, por lo tanto, los componentes reconfigurables que utilizan esta API, puede ser reemplazados, añadidos o eliminados del sistema, por el mismo tipo de componente en tiempo de ejecución, es decir, sólo se actualiza el código del componente, mientras que las interfaces y estado siguen siendo los mismos, lo que permite las actualizaciones de software, posteriores al despliegue de los nodos.

LooCI (Hughes et al., 2012) es un middleware diseñado para soporte de redes de sistemas embebidos en general, proporciona un modelo de fácil uso, independiente de la plataforma, con programación basada en componentes, que soporta la reconfiguración en tiempo de ejecución de aplicaciones distribuidas desplegados en una red específica. Esto se logra a través de un modelo basado en eventos, que permite el desacoplamiento de los componentes que comprenden una aplicación, mediante los eventos de publicación/suscripción definidos por interfaces requeridas $\mathrm{y}$ proporcionadas entre sí, a través de un bus de eventos compartido, con tipos de eventos identificados de forma única y global. Su arquitectura permite una gestión separada en tiempo de ejecución de los componentes de software, de su interconexión. Es decir, los componentes individuales se pueden desplegar en los nodos, de forma segura en tiempo de ejecución, y posteriormente pueden ser preservados en función de su estado, según lo indicado por los comandos de eventos recibidos por medio del bus, concernientes al control, la introspección y al enlace de los componentes.

FamiWare (Gámez \& Fuentes, 2011) es una familia de middleware configurable basada en eventos, con arquitectura flexible frente a las necesidades de las aplicaciones de Inteligencia Ambiental (AmI). Trabaja con diferentes dispositivos que van desde relativamente potentes (por ejemplo, los teléfonos inteligentes), hasta los de recursos limitados (por ejemplo, WSN motas). Permite la personalización en tiempo de diseño, y el despliegue de una manera automática del middleware en los nodos de la red, empleando un proceso basado en modelos, descrito por Software Product Line (SPL) (Pohl, Böckle, \& Linden, 2005). Alta flexibilidad de composición se logra con la adopción del paradigma de publicación/suscripción basado en eventos, utilizado para realizar la conexión entre los elementos del middleware y las aplicaciones, al tiempo que se apoya además la reconfiguración de auto-adaptativa de una implementación de middleware, con la provisión de servicios de monitoreo y de contexto, que inician el proceso de reconfiguración bajo la detección de un contexto de datos específico.

TinyLIME (Curino et al., 2005) y TeenyLIME (Costa, Mottola, Murphy, \& Picco, 2007), ambos son middleware con enfoque de datos compartidos, los cuales están basados en una abstracción de espacio de tuplas (Gelernter, 1985), según la cual los datos son representados por estructuras elementales llamadas tuplas, formando un espacio de memoria compartida denominada espacio de tuplas. TinyLIME adopta un esquema operativo para el desarrollo de aplicaciones, donde los dispositivos en las WSN son accesados por los nodos tipo móvil sink (sumideros móviles), en el rango de 
distancia de un salto, formando espacios de tuplas con los datos recogidos por el nodo sink, y luego estos son compartidos con otros dispositivos móviles (por ejemplo, PDA). Al contrario del papel confinado de los nodos en las WSN como productores de datos simples, TinyLIME y TeenyLIME, en esencia, reemplazan el paradigma de comunicación simple de intercambiar mensajes entre los nodos, con el paradigma que proporciona una abstracción de espacio de tuplas, manteniendo la lógica de aplicación y la implementación de espacio tupla y el control a nivel de nodo.

Agilla (Fok, Roman, \& Lu, 2009) es un middleware modular para WSN, basado en una abstracción de programación de máquina virtual y el paradigma de programación de agentes móviles, para apoyar el desarrollo de aplicaciones, proporcionando flexibilidad para realizar modificaciones de acuerdo a los diferentes requerimientos de usuario en el tiempo, y permitir la adaptación de las aplicaciones en los dinámicos entornos de las WSN. Las aplicaciones están compuestas por agentes móviles que se mueven o clonan de sí mismos a través de los nodos con el apoyo de la información de localización proporcionada por la lista de vecinos que mantienen todos los nodos con el objetivo de ejecutar tareas solicitadas por la aplicación, y coordinar con los demás el uso del modelo de memoria de espacio de tuplas, que permite compartir y reaccionar de forma asíncrona a los datos que aparecen formados en los espacios de tuplas compartidos de los nodos. La migración de los agentes que contienen su código y su estado, conduce a la ejecución concurrente de múltiples agentes en un solo nodo, permitiendo de esta manera el soporte de múltiples aplicaciones simultáneamente, que pueden ser desplegadas en la red por diferentes usuarios. En cuanto a la ejecución de los agentes, que están escritas en un lenguaje tipo assembler, el modelo de ejecución está basado en el stack, de acuerdo con el cual los agentes tienen sus propio stack y registros, que constituyen su estado, y que utilizan un conjunto de instrucciones, siendo la mayoría representada por bytes individuales, lo que resulta en la reducción de lo transferido cada vez, por un tamaño más pequeño de código de agente y en consecuencia una rápida reprogramación de la aplicación y adaptación dinámica.

Servilla (Fok, Roman, \& Lu, 2012) es un middleware modular orientado a servicios, que toma ventaja de la heterogeneidad de las WSN, permitiendo la ejecución de scripts en las capas superiores de la red. Basándose en Agilla, una aplicación se compone de tareas (en semejanza a los agentes móviles de Agilla), escrita de una manera más fácil por medio de un script con un lenguaje al estilo C, el cual es compilado en código de bytes con el objetivo de ser ejecutado en una máquina virtual, mientras que la coordinación entre ellos se basa en un modelo de espacios de tuplas, que se extiende por la incorporación de Service Provisioning Framewaork (SPF), lo que permite el despliegue de aplicaciones más complejas y flexibles. Servilla desacopla del nivel de aplicación, la lógica de aquellas partes que se refieren a capacidades software y hardware específicos de los dispositivos subyacentes en las WSN, a fin de lograr la independencia de plataforma. Al mismo tiempo estas capacidades, pueden ser utilizadas por las tareas de la aplicación, proporcionado a nivel local o remoto, detección y ejecución de servicios gestionados por el SPF. Teniendo en cuenta la 
arquitectura modular de Servilla, esta permite el despliegue de nodos, que debido a las limitaciones de recursos pueden implementar partes del middleware, lo que provoca tres tipos de roles en los nodos: el soporte a cualquiera de todos los potenciales de middleware, o todos excepto para provisión de ejecución del servicio, o sólo proporcionar publicación y ejecución de servicios.

TinySOA (Avilés-López \& García-Macías, 2009) utiliza una arquitectura orientada a servicios que facilita el acceso a las redes inalámbricas de sensores, proporcionando una API sencilla y orientada a servicios, para el desarrollo de aplicaciones. Integra las WSNs con las redes IP a través de gateways, en los cuales reside el proveedor de servicios, permitiendo que el proceso de desarrollo de aplicaciones sea extiendo hasta el uso de servicios web. En cuanto a los servicios de TinyOS, estos son considerados como unidades computacionales con identificadores únicos, que expresan alguna capacidad funcional de la infraestructura de red subyacente. Estas capacidades pueden ser invocadas enviando consultas orientadas a servicios, hacia cualquiera de los gateway de la WSN, o a los nodos individuales de la red, definiendo los datos apropiados y la información de control de eventos. La provisión de servicios es implementada por la infraestructura de middleware de la WSN, basada en el modelo de comunicación de publicación/suscripción, entre el nodo y el gateway.

Considerando los requerimientos y la similar naturaleza de funcionamiento de las WSAN, y después de la amplia revisión sobre el diseño y desarrollos de middleware para WSAN, se pueden identificar tres tendencias importantes que pueden contribuir a futuros esfuerzos de diseño e implementación de middleware, dichas tendencias son: paradigma de agentes móviles, orientado a servicios e ingeniería model-driven. Hay que enfatizar que a pesar del amplio rango de plataformas, no existe una solución global en este tema. En este contexto, el diseño de middleware adecuado comprende un campo de investigación importante, con el objetivo de satisfacer tales desafíos multifacéticos y dinámicos.

\section{Tecnologías de alto nivel}

En cuanto a las tecnologías de alto nivel, la aparición de IoT y conceptos subyacentes (Smart Objects), indican que el futuro de las comunicaciones estarán dominadas por las redes all-IP. La utilización de redes basadas en IP trae consigo un conjunto de beneficios, entre los cuales debemos destacar la posibilidad de utilizar Web Services. En este sentido, el CoRE Working Group de la IETF, ha propuesto un nuevo protocolo para servicios web en redes de Smart Objects. Este protocolo es denominado Constrained Application Protocol (CoAP) (Shelby, Hartke, \& Bormann, 2012), y ha sido desarrollado para ejecutarse en ambientes especiales, que incluyen dispositivos con recursos limitados (alta taza de pérdida de paquetes, limitada autonomía energética y capacidades de hardware, entre otros).

CoAP es un protocolo optimizado para redes con recursos limitados, típicas del IoT o en aplicaciones de Machine-to-Machine (M2M). CoAP está constituido por un subconjunto de funcionalidades del HyperText Transport Protocol (HTTP), las cuales 
han sido rediseñadas tomando en consideración el bajo poder de procesamiento y las restricciones de consumo energético, de los dispositivos tales como sensores y actuadores. Este protocolo está organizado en dos subcapas (Request/Response y Transacción), y está construido encima del User Datagram Protocol (UDP). Utiliza una pequeña cabecera binaria de longitud fija (4 bytes), seguida de unas opciones binarias muy compactas, también soporta algunas codificaciones estándar de la carga como Extensible Markup Language (XML) (Colitti, Steenhaut, De Caro, Buta, \& Dobrota, 2011).

Aunque CoAP es un trabajo en progreso, existen varias implementaciones de código abierto que ya están disponibles, incluyendo dos sistemas operativos para WSAN, los bien conocidos Contiki (Kovatsch, Duquennoy, \& Dunkels, 2011) y Tiny Os (Paczesny, Tajmajer, Domaszewicz, \& Pruszkowski, 2012). El rendimiento de CoAP es óptimo en entornos con infraestructuras all-IP. Cuando es necesaria coexistencia de soluciones basadas en IP, con tecnologías de sistemas heredados, una alternativa viable podría ser el uso de middleware de alto nivel, capaz de mejorar la flexibilidad, interoperabilidad y apoyar a los distintos tipos de aplicaciones.

Aunque existen varios protocolos propuestos para la comunicación con las WSAN, que incluyen Constrained Application Protocol (CoAP) y Message Queue Telemetry Transport (MQTT), este último aparece como una alternativa válida para resolver algunos problemas, proporcionando una solución eficiente y reduciendo la sobrecarga en la conexión de los dispositivos con recursos limitados.

MQTT ("MQTT," 2014) fue creado por IBM y Eurotech, como un protocolo de conectividad extremadamente ligero para publicación/suscripción, y transporte de mensajería basado en TCP/IP. La publicación/suscripción se gestiona basándose en la noción de tópicos. MQTT permite a los dispositivos abrir una conexión y mantenerla abierta consumiendo muy poca energía, además de entregar los mensajes con una sobrecarga mínima, características requeridas en la comunicación de dispositivos de recursos energéticos limitados. MQTT ha sido recientemente estandarizado por OASIS (“MQTT Version 3.1.1 (OASIS Standard)," 2014).

La fiabilidad de los mensajes MQTT está definida por tres niveles de calidad de servicios (QoS). QoS nivel 0, significa que un mensaje se entrega como máximo una vez y no se requiere acuse de recibido. Nivel 1 de QoS, significa que cada mensaje se entrega al menos una vez y no requiere acuse de confirmación de la recepción del mensaje. El QoS de nivel 2, un mecanismo de saludo de cuatro vías es requerido para la entrega de un mensaje exactamente una vez.

IEEE 802.15.4 proporciona las funciones de capa física y de capa MAC, sobre las que MQTT puede proveer las funcionalidades de capa de transporte. MQTT se prefiere sobre todo, debido a su bajo consumo de energía, bajos requerimientos de ancho de banda, alta escalabilidad y baja sobrecarga. Debido a estas ventajas MQTT ha sido ampliamente implementado para el control industrial (SCADA), la señalización ferroviaria (Delta-rail), la vigilancia del transporte en tiempo real (Red Funnel), 
automatizaciones, la atención sanitaria y aplicaciones en las redes sociales (Facebook) (Wood \& Robson, 2012). Sin embargo, MQTT requiere una conexión TCP/IP, que provee unas capacidades de conexión sin pérdidas de ordenamiento, que es demasiado compleja para un muy simple y pequeño dispositivo sensor de bajo costo. Además, no es factible utilizar cables en el escenario de una red de baja potencia. En atención a este tema y a otros problemas relacionados con las redes inalámbricas de sensores y actuadores, se ha desarrollado MQTT-SN. MQTT-SN puede ser considerada una versión de MQTT adaptada a los ambientes de comunicaciones inalámbricas. A diferencia de MQTT, en los sistemas MQTT-SN, los nodos finales se conectan al gateway utilizando el protocolo MQTT-SN sobre medios inalámbricos y el Gateway es conectado al servidor/broker utilizando el protocolo MQTT sobre una red cableada. Además, MQTT-SN usa conexión UDP principalmente porque se adapta mejor a los requerimientos de transmisión de los mensajes simples de MQTT-SN. De igual forma UDP es más rápido, simple, eficiente y mucho más ligero que TCP sobre enlaces inalámbricos, y con el compromiso de fiabilidad adaptado por las aplicaciones.

Las principales diferencias entre CoAP. MQTT y MQTT-SN están resumidas en la Tabla 1.

Tabla 1: Diferencias entre protocolos de alto nivel: MQTT, MQTT-SN y CoAP

\begin{tabular}{|c|c|c|c|}
\hline & CoAP & MQTT & MQTT-SN \\
\hline $\begin{array}{c}\text { Capa de } \\
\text { Aplicación }\end{array}$ & $\begin{array}{l}\text { Una capa simple con } \\
\text { dos subcapas } \\
\text { conceptuales (Mensajes } \\
\text { y Solicitud/Respuesta) }\end{array}$ & Capa completa & Capa simple básica \\
\hline $\begin{array}{c}\text { Capa de } \\
\text { Transporte }\end{array}$ & UDP & TCP & UDP \\
\hline Confiabilidad & 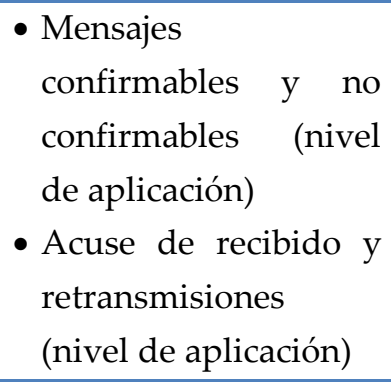 & $\begin{array}{l}\text { - Tres niveles de } \\
\text { calidad de servicio } 3 \\
\text { QoS (nivel de } \\
\text { aplicación) } \\
\text { - Acuse de recibido y } \\
\text { retransmisiones } \\
\text { (nivel de transporte) }\end{array}$ & $\begin{array}{l}\text { - Tres niveles de calidad } \\
\text { de servicio } 3 \text { QoS } \\
\text { (nivel de aplicación) }\end{array}$ \\
\hline $\begin{array}{l}\text { Paradigma de } \\
\text { Comunicación }\end{array}$ & $\begin{array}{l}\text { - Solicitud/Respuesta } \\
\text { nativa } \\
\text { - Publicación/Suscripci } \\
\text { ón adaptada }\end{array}$ & $\begin{array}{l}\text { - Publicación/Suscripci } \\
\text { ón nativa }\end{array}$ & $\begin{array}{l}\text { - Publicación/Suscripció } \\
\text { n nativa }\end{array}$ \\
\hline
\end{tabular}




\section{Integración con Internet}

La inminente integración de las WSAN a la web, impulsada por el paradigma del IoT, trae consigo nuevos desafíos. Las WSAN han sido diseñadas ad-hoc atendiendo a los entornos y aplicaciones, como sistemas monolíticos. No hay reglas, ni estándares que se hayan considerado en el diseño de arquitecturas para las WSAN, por lo tanto, éstas solo responden a los objetivos específicos de aplicaciones concretas, y a las capacidades de la red subyacente.

La evolución de las nuevas tecnologías y los requerimientos de las aplicaciones modernas (como por ejemplo: el IoT), requieren que las WSAN propietarias y los nuevos despliegues, ofrezcan conectividad con Internet. En este punto, los gateway o proxys pasan a jugar un rol importante, permitiendo la interacción entre host en Internet y los nodos en las redes de sensores y actuadores. IPv6 sobre Low-power Personal Area Networks (6LoWPANs) habilita la compatibilidad con IPv6 en redes inalámbricas de sensores, proporcionando a los nodos la conectividad con Internet. Sin embargo, se necesitan nuevos gateway a fin de que sea posible la conectividad de extremo a extremo entre nodos sensores y host en Internet.

Arquitecturas de Gateway para WSAN han sido propuestas en la literatura. Existen implementaciones (Dun-fan, Liang-liang, \& Wang, 2009; Qiu, Heo, \& Choi, 2009; Steenkamp, Kaplan, \& Wilkinson, 2009) orientadas a configuración remota y recuperación de información de las WSAN, que utilizan interfaces basada en tecnologías web, para la presentación de resultados a los usuarios. Sin embargo, su desventaja es que utilizan protocolos propietarios para conectarse al nodo sensor, por lo tanto, el gateway actúa como proxy, impidiendo la interacción directa entre los clientes y los nodos sensores. El uso de protocolos estándar en nodos de sensores, tales como IPv6, permite la comunicación end-to-end con ellos, y reduce la complejidad del gateway.

Existen otros trabajos relacionados que desarrollan gateways para el intercambio de información entre los nodos sensores basados en 6LowPAN, desplegados en distintos escenarios. En (Jiang, Dawson-Haggerty, Dutta, \& Culler, 2009) se propone un sistema de gestión de energía eléctrica, basado el desarrollo de una red inalámbrica en malla, en donde cada nodo está dotado de un stack completo de IPv6/6LowPAN, e incluye un router edge, quien proporciona conectividad con otras redes Ip. En la capa de aplicación se reciben y almacenan las lecturas en una base de datos, y se utiliza un servidor web para visualización.

Una arquitectura de WSN con gestión de movilidad para entornos hospitalarios es propuesta en (Jara, Zamora, \& Skarmeta, 2009), la arquitectura consiste en Gateway locales, dotados con 6LowPAN y con otras tecnologías (Ethernet, Cellular, ZigBee or Bluetooth) que le permiten conectarse con otras redes. Estos Gateway locales son sink móviles, tienen el objetivo de recolectar la información de los dispositivos adyacentes (sensores en pacientes) y comunicarse con el sistema de información del hospital, quien actúa como gateway global. 
Un sistema de soporte para gestión de desastres es presentado en (Mayer \& Fritsche, 2006). La arquitectura consiste en una red de sensores habilitados con Ipv6 conectados a una estación de centralizada, mediante diferentes tecnologías WAN. La arquitectura incluye un gateway con capacidades de comunicación con las WSAN vía Ipv6/6LowPAN, y por otro lado capacidades de comunicación con redes WAN con diferentes interfaces, WLAN, GPRS, SatLink (Enlace Satelital), basadas en Ipv6. Este trabajo también presenta un análisis de los beneficios de las WSAN basadas en IP y las no IP. Adicionalmente, analiza el tema de gestión de la movilidad en los enlaces WAN.

El desarrollo de gateway especializados, es una alternativa válida para lograr la integración de las WSAN con las redes globales Ip. Después de la revisión de la literatura, es necesario analizar los esfuerzos necesarios para el desarrollo de gateway, versus la implementación de arquitecturas Ip end-to-end y los beneficios subyacentes. Con el inminente advenimiento de los nuevos estándares, y los requerimientos de las cada vez más sofisticadas aplicaciones sobre las WSAN, es de esperarse que las WSAN sean en poco tiempo redes Ipv6.

\section{Problemática de recolección, almacenamiento y procesado de datos}

La recolección de datos en las redes inalámbricas de sensores y actuadores, desplegadas en zonas con comunicaciones limitadas sigue siendo un desafío bajo investigación (Wang \& Liu, 2011). Los nodos sensores y actuadores cuentan con recursos limitados (buffer de almacenamiento, fuente de alimentación, rango de comunicación), por lo tanto, en WSAN aisladas es necesaria la recolección periódica de los datos, para evitar pérdidas.

Por otro lado, el volumen de datos, las fuentes de datos heterogéneas y los limitados recursos de procesamiento de las WSAN, trae como consecuencia que los datos no puedan ser almacenados, analizados, gestionados y convenientemente aplicados, para proveer servicios a los sistemas de usuario final. Adicionalmente, el incremento en la demanda de acceso a sus datos, por parte de una creciente comunidad de usuarios de Internet (Ting-hui et al., 2010), sugiere una arquitectura homogénea, interoperable, extensible y accesible por todos los interesados.

\section{Conclusiones y relación con los objetivos expuestos en la Sección 2}

La exhaustiva revisión a las principales tecnologías utilizadas en la WSAN, ha permitido detectar que es necesario el desarrollo de soluciones abiertas y estandarizadas, que permitan la integración de tecnologías IP y no IP. También se requiere de soluciones middleware de alto nivel, para mejorar la flexibilidad, interoperabilidad y brindar soporte a los distintos tipos de aplicaciones emergentes.

Después del estudio anterior, se identifican tres enfoques principales en donde se agrupan los middleware planteados, estos son: paradigma de agentes móviles, orientado a servicios e ingeniería model-driven. Hay que enfatizar que a pesar del amplio rango de plataformas, no existe una solución global en este tema. Es 
importante la provisión de un middleware con el objetivo de satisfacer los desafíos multifacéticos y dinámicos. El diseño del middleware debe contemplar la gestión de la ejecución de servicios móviles distribuidos, la comunicación e integración de dispositivos de distintas tecnologías, y tenga en cuenta factores como los entornos remotos, las comunicaciones limitadas, el volumen de datos, las fuentes de datos heterogéneas y los limitados recursos de procesamiento de los dispositivos (Objetivo $\# 3)$.

Las nuevas tecnologías emergentes de alto nivel se enfocan básicamente a brindar soporte a las capa superiores, lo que sugiere la posibilidad de integración de las WSAN con infraestructuras globales, como Internet. Los protocolos MQTT, MQTT-SN y $\mathrm{CoAP}$, han sido analizados como los principales exponentes de las tecnologías de capas superiores. La caracterización y diferenciación de ellos nos permite determinar su enfoque, confiabilidad, protocolo de transporte y las prestaciones de la capa de aplicación, para determinar cuál es el más adecuado en cada segmento de la arquitectura.

\subsection{DTN: Hacia una solución para entornos con comunicaciones limitadas}

En el escenario de las WSAN desplegadas en sitios remotos, los nodos cluster head recolectan los datos de sus nodos sensores subyacentes, pero no tienen conexión permanente con la estación base que almacena los datos. En estos casos las WSAN se vuelven incapaces de proporcionar servicios requeridos por los usuarios o pierde incluso totalmente su capacidad de proporcionar cualquier servicio en absoluto. La desconexión prolongada de los sensores o grupo de sensores, y considerando los perfiles de hardware con que cuentan estos dispositivos (buffer de almacenamiento, memoria, batería, capacidad de procesamiento y comunicación) pueden provocar la pérdida los datos adquiridos, convirtiendo la WSAN en una red no funcional o escasa, aunque sus sensores o actuadores permanezcan en funcionamiento. Una posible forma de superar tales desafíos es proporcionar una comunicación alternativa, a través de nodos móviles que permitan apoyar con un enfoque oportunista, la conexión entre las WSAN aisladas y una infraestructura de red con conectividad permanente de extremo a extremo.

\section{Soluciones Ad-Hoc}

Existen varios trabajos propuestos, uno de ellos son los denominados sink móviles, es decir, tener uno o varios sink que se muevan de forma periódica o eventualmente entre las redes de sensores aisladas coleccionando los datos. En los enfoques que utilizan este concepto, los nodos sensores esperan hasta que un móvil sink aparezca en su rango de comunicación para entregar datos (Marinho, de Freitas, Lustosa da Costa, de Almeida, \& de Sousa, 2013). En (Pignaton de Freitas et al., 2010) proponen un conjunto de sinks móviles tipo Unmanned Aerial Vehicles (UAVs), de tal forma que cubran toda el área de despliegue de las WSAN, esto permite que los nodos siempre 
encuentren un nodo sink para entregar sus datos. En (Mikhaylov \& Tervonen, 2013) proponen la utilización de mobile ferries (MF) que es un elemento móvil que transfiere los datos entre las WSAN aisladas y los nodos tipo sink, a través de su trayectoria de movimiento. En otras palabras los MF aprovechan su movilidad y capacidad de almacenamiento temporal, para recolectar y transportar los datos hasta encontrar un sumidero en su rango de comunicación.

Por otro lado, se han planteado trabajos basados en la aplicación de estrategias multiple input multiple output (MIMO) en las islas de nodos de sensores, con el objetivo de extender su rango de comunicación. Con esta extensión, estos grupos de nodos son capaces de llegar a los sink móviles (UAVs) más lejanos, por lo tanto es posible reducir el número de sink móviles para cubrir una zona, así como mitigar las limitaciones en su trayectoria de movimiento (Marinho et al., 2013).

\section{Alternativas relacionadas con DTN}

Otro enfoque es el que está vinculado con la recolección de datos mediante redes tolerantes a fallos y retardos (Delay Tolerant Nerwork - DTN). Las DTN admiten desconexiones y largos retardos de comunicación, características requeridas por naturaleza en los entornos de WSAN remotas o aisladas. Las DTN's permiten la transferencia de datos donde los protocolos de red convencionales no pueden, porque la conectividad continua de extremo a extremo no está siempre disponible. Si bien el Bundle Protocol (BP), se ha establecido como el protocolo estándar DTN, en muchas áreas de aplicación como redes inalámbricas de sensores y actuadores, a menudo utilizan protocolos propietarios con un subconjunto de las características de BP. El BP ofrece muchas capacidades requeridas en el contexto de las WSAN remotas. Una de las más importantes es que utiliza estrategias de persistencia de almacenamiento, para mantener el estado de las comunicaciones (Finite-State Machine FSM), permitiendo reanudar una transferencia bidireccional de datos, en el estado anterior en donde se interrumpió. Esta funcionalidad permite ofrecer soporte eficiente a sistemas de comunicación basados en eventos. Un ejemplo de este enfoque, son los sistemas diseñados bajo el paradigma de publicación/suscripción, debido a su adaptación natural a entornos de comunicaciones asíncronas, muy comunes en el contexto de las WSAN remotas (Sheltami, Al-Roubaiey, Mahmoud, \& Shakshuki, 2015).

Existen una multitud de escenarios de aplicación para las Delay Tolerant Wireless Sensor Networks (DT-WSN), en adelante se resumen algunos casos representativos de la literatura y sus mayores contribuciones.

ZebraNet (Juang et al., 2002) tiene como objetivo rastrear la vida silvestre en Kenia, colocando nodos equipados con GPS en los animales. Los nodos intercambian datos de forma inalámbrica, permitiéndoles a los investigadores recuperar la mayor cantidad de piezas de información como sea posible, mediante la trazabilidad de sólo un subconjunto de los animales. El retardo de los datos es irrelevante, pero una alta tasa de entregas de muestras es importante. 
Seal-2-Seal (Lindgren, Mascolo, Lonergan, \& McConnell, 2008) es un protocolo tolerante a retardos. Enfocado principalmente en registrar los contactos entre los animales en su hábitat natural. El protocolo utiliza un mecanismo de resumen eficiente de datos, para reducir la cantidad de información que necesita ser transmitida, reduciendo así el consumo de energía. Se ha implementado para Contiki, y evaluado en simulaciones.

SeNDT (McDonald, Geraghty, Humphreys, Farrell, \& Cahill, 2007) es para monitorear la calidad del agua y la emisión de ruido, utilizando nodos sensores y estrategias de store-and-forward para recolectar los datos. SeNDT utiliza una implementación de Licklider Transmission Protocol (LTP) por encima del stack de IP y está implementado para sistemas operativos Linux.

LUSTRE (Selavo et al., 2007) tiene como finalidad el monitoreo de parámetros ambientales para ser analizados por los ecologistas. Este utiliza un enfoque de múltiples capas con dos elementos separados. En la red de sensores, LUSTRE utiliza un sistema de log basado en la escucha que los nodos realizan en la transmisión de radio, y guardan los datos en el almacenamiento local persistente. Además, LUSTRE utiliza un enfoque DTN, para la conectividad en el back-end, sobre los enlaces intermitentes. Esto permite al back-end consultar los nodos sobre los datos que se perdieron durante el tiempo de inactividad del enlace.

Vineyard Computing (Burrell, Brooke, \& Beckwith, 2004) presenta una WSN, en el que los sensores en un viñedo periódicamente muestrean la temperatura, y mulas de datos (es decir, los trabajadores, los perros, etc.) llevan nodos para recoger los datos.

Un sistema basado en DTN para la recolección de datos de sensores en una granja es propuesto en (Ochiai, Ishizuka, Kawakami, \& Esaki, 2010), básicamente el sistema recolecta los datos de los sensores desplegados en sitios remotos sin utilizar la red de los operadores de servicios de telecomunicaciones. Este considera que los sensores en el campo pueden estar separados por grandes distancias, y que su densidad puede estar en función del área de la superficie, por lo tanto la utilización de dispositivos provistos con radiocomunicación satelital son prohibitivos por temas de costo de los equipos y del propio del servicio de comunicación. El enfoque DTN, sumado al aprovechamiento de la movilidad de los tractores durante el trabajo diario, permiten la recolección de los datos especialmente en zonas aisladas o remotas, asumiendo que los tractores y sensores cluster head están equipados con un dispositivo de radio de corto rango y capacidades DTN. El dispositivo recolector a bordo del tractor u otra entidad, al entrar en contacto con los cluster head hace uso de comunicación ad-hoc para recuperar los datos que posteriormente entrega al data server, al llegar a la posición de origen.

La conectividad continua de extremo a extremo no siempre está presente en los ambientes de aplicación de las WSAN, pero se pueden utilizar las técnicas de almacenamiento y envío (store-and-forward) para hacer frente a la conectividad intermitente. Un ejemplo es el trabajo propuesto por (Pottner, Busching, von Zengen, 
\& Wolf, 2012), donde se propone el Data Elevator, este utiliza una implementación propia de BP para las plataforma de WSN en conjunción con BP para Pc, con el objetivo de monitorear la temperatura exterior. El Data Elevator utiliza el enfoque de storecarry-and-forward, en el cual un ascensor realiza la función de mula de datos (MULE Mobile Ubiquitous LAN Extensions), para transportar los datos desde el techo hasta el laboratorio ubicado en oro edificio cercano. Este enfoque aplica el BP estándar en WSANs y evita la sobrecarga de las capas 3 y 4.

\section{Conclusiones y relación con los objetivos expuestos en la Sección 2}

En el apartado anterior se han planteado un conjunto de investigaciones orientadas principalmente en resolver el problema de la recolección de datos en entornos de WSAN remotas, que tienen como denominador común la ausencia de enlaces de comunicación permanentes de extremo a extremo.

El problema se aborda desde dos perspectivas, la primera está enfocada hacia soluciones ad-hoc. Estos trabajos consideran entidades móviles o conjuntos de ellas, que recolectan los datos de las WSAN pervasivas en escenarios concretos, haciendo énfasis en la movilidad y trayectoria de los sumideros o en nodos móviles que replican los datos recolectados hacia sumideros distantes. Sin embargo, estas investigaciones asumen que en los contactos las transferencias de datos se completan, es decir, que los datos siempre se transmiten en su totalidad. No se contemplan mecanismos de reanudación de transferencias en el estado en que fueron interrumpidas, por lo tanto, cuando esto ocurre se debe volver a iniciar la transferencia desde el inicio. Otras soluciones ad-hoc están basadas en aspectos de hardware para mejorar el rango de cobertura de los nodos, sin considerar las restricciones propias de los dispositivos con recursos limitados. Este enfoque no considera soluciones estándares, lo que dificulta la generalización de las soluciones, que permitan la integración de WSAN heterogéneas.

La otra perspectiva analizada, consiste en la utilización las redes tolerantes a retardos (DT-WSN). Después de la revisión de los trabajos orientados con este enfoque, se llega a la conclusión que hay una gran multitud de escenarios de aplicación de las DT-WSN, y que además, existe la necesidad de comunicaciones tolerantes a retardos. En este ámbito se han desarrollado varios trabajos que se basan ya sea en protocolos no estandarizados, que son especialmente diseñados o se implementan utilizando nodos mucho más potentes que los presentes en las WSAN. En general, los protocolos estándar tienen la desventaja de que no están optimizados para un caso de uso específico $\mathrm{y}$, por lo tanto, tienen una sobrecarga mayor en comparación con protocolos especializados. Sin embargo, el uso de protocolos estándar reduce significativamente la curva inicial de aprendizaje para configurar una red, ya que las implementaciones están fácilmente disponibles. Eso también permite la integración de redes de sensores utilizando protocolos estándar en redes de back-end existentes, ya que existen pilas de protocolos para esos sistemas. Además, los protocolos estándar por lo general ya han resuelto muchos de los problemas. 
Existen investigaciones que demuestran que las DTN, son una alternativa factible en el contexto de las WSAN. Como se señaló anteriormente el Bundle Protocol (BP) se ha establecido como el protocolo estándar DTN, y una de sus funcionalidades más importantes es la utilización de mecanismos de persistencia para almacenar el estado de las comunicaciones, lo que posibilita reanudar una transmisión de datos, en el estado donde se suspendió.

Una posible forma de superar tales desafíos es proporcionar una alternativa de comunicación estándar, basada en nodos móviles con capacidades DTN, que permitan ofrecer soporte a la comunicación entre las WSAN aisladas y una infraestructura de red con servicio continuo de comunicación (Objetivo \#2).

Relacionado con la gestión de servicios tolerantes a retardos. La revisión bibliográfica nos permite contribuir con la: Definición de un framework de gestión de servicios basado en cloud, que integre las tecnologías DTN para proveer mecanismos de soporte a servicios tolerantes a disrupciones y retardos, desplegados en redes inalámbricas de sensores y actuadores con comunicaciones limitadas (Objetivo \#6).

\subsection{Aplicaciones y servicios}

Las WSAN tienen un amplio conjunto de escenarios de uso, sin embargo, las tecnologías emergentes como el IoT han provocado un crecimiento exponencial en su ámbito de utilidad o aplicación. Las WSAN son un componente fundamental en el paradigma del Internet de las Cosas (Internet of Things - IoT), ya que estas permiten mayor ubicuidad de la tecnología, obteniendo información detallada del entorno (Tinghui et al., 2010). La visión del Internet del Futuro considera al Internet of Things (IoT) como una parte fundamental e integradora. El IoT puede ser descrita como la interconexión de smart objects (Ejemplo: smart-phones, Internet TVs, sensores y actuadores) a la World Wide Web (WWW) donde los dispositivos tienen enlaces inteligentes, permitiendo nuevas formas de comunicación entre las cosas y las personas, y entre ellos mismos (Piyare et al., 2013). Por anteriormente planteado, es necesaria una plataforma que proporcione soporte para el desarrollo de servicios o aplicaciones, que considere los requerimientos específicos de los nuevos escenarios en donde las WSAN representan recursos básicos para su desarrollo.

Hoy día uno de los framework costo-efectivo y versátil más utilizados, que proporcionan datos de en tiempo real a los usuarios, en cualquier momento y lugar, son los web services en la cloud. La cloud consiste en hardware, redes, servicios, almacenamiento, e interfaces que permiten la entrega de computación como servicio (Perumal, Rajasekaran M., \& Ramalingam, 2012). Los servicios web son la forma más abierta e interoperable de brindar servicios remotos, comunicación entre aplicaciones y entre los propios servicios (Piyare et al., 2013). La computación en cloud, reduce el acoplamiento entre las aplicaciones y los recursos, permitiendo una eficiente gestión de los recursos, además mejora la ubicuidad de acceso a la información y la pervasividad de los servicios y aplicaciones. Las tecnologías cloud, pueden eficientemente 
almacenar, procesar, analizar y entregar, aplicaciones y servicios basados en las capacidades de las WSAN. La conexión de las islas de WSAN con redes externas, mediante servicios web, también ha sido sugerida por investigadores (Villaverde, Pesch, De Paz Alberola, Fedor, \& Boubekeur, 2012).

En estos nuevos escenarios donde las WSAN juegan un papel fundamental, nuevas aplicaciones serán necesarias, y su diseño debe considerar requerimientos particulares de varios factores que condicionan su desarrollo y despliegue. Por un lado están los requerimientos de las WSAN, es decir, las aplicaciones deben considerar los dispositivos (sensores/actuadores) con recursos limitados que conforman las WSAN. En el siguiente orden están los desafíos asociados con las comunicaciones limitadas, entre las islas de WSAN y las redes externas con enlaces permanentes de extremo a extremo. Este desafío es de particular importancia para las aplicaciones y servicios, ya deben tomar en cuenta que no siempre encontrarán una comunicación continua entre los nodos que participan en la ejecución de los servicios, y adicionalmente deben soportar largos periodos de espera. De igual forma, se deben superar los requerimientos de comunicación impuestos por las arquitecturas, los cuales se tratan con detalle en la Sección 3.4.

El modelo SOA (Service Oriente Architecture) es la solución aceptada comúnmente para el diseño y desarrollo de servicios (GISWG, 2004). De esta forma, el diseño, desarrollo e implementación de servicios es posible gracias a las tecnologías Web. Sin embargo, la interoperabilidad de servicios en SOA no está limitada únicamente a servicios Web (Franco, Bas, \& Esteban, 2009) (Demirkan et al., 2008). En este tipo de arquitectura, las fuentes de información, y la lógica de ejecución pueden convertirse en servicios modulares o atómicos, para el control y la gestión (Cheng, Law, Bjornsson, Jones, \& Sriram, 2010).

Para soportar y desarrollar servicios basados en SOA se deben describir con un alto nivel de abstracción (Zhang, Ying, Cao, \& Jia, 2006). Por tanto, el modelado de servicios juega un rol importante en aplicaciones basadas en SOA (Tohidi, 2011). Basado en la especificación WSDL, el estándar UDDI (Universal Description Discovery \& Integration) (OASIS, 2004), permite establecer un directorio de servicios definiendo para cada uno de ellos un conjunto de meta-información. Este directorio de servicios soporta la descripción y el descubrimiento de otros proveedores de servicios Web, los servicios Web disponibles, y también las interfaces que deben ser utilizadas para consumir la funcionalidad de esos servicios.

Utilizando UML (Unified Modeling Language) y la especificación basada en UML para la descripción de servicios Web: SoaML (Service Oriented QArhcitecure MOdeling Language) (Elvesæter, Berre, \& Sadovykh, 2011), se presenta un meta-modelo para la especificación y el diseño de servicios en una arquitectura orientada a servicios. Soporta de una manera estándar establecer estrategias colaborativas de utilización y compartición de servicios, necesarias para la definición de mecanismos de gestión de servicios más complejos como la delegación y la resolución. 
Otro lenguaje utilizado con frecuencia en la literatura es SPEM (Software Process Engineering Meta-model), que es una especificación del OMG para la definición de procesos de desarrollo software así como sus componentes. Su mayor objetivo es cubrir un gran rango de métodos de desarrollo y procesos de distintos estilos, niveles de formalismo y modelos de gestión de ciclo de vida, entre otros. SPEM ha sido utilizado en numerosos trabajos para el modelado formal del ciclo de vida de servicios (Rebollo, Mellado, \& Fernandez-Medina, 2015), así como en otros destacados trabajos de tesis (Alcarria Garrido, 2013).

Para una ejecución continua de servicios en entorno disruptivos se debe presentar especial atención a los trabajos relacionados con la ejecución continua de servicios en entornos móviles (Ramon Alcarria, Robles, Morales, López-de-Ipiña, \& Aguilera, 2012). La ejecución continua de servicios o el service roaming (Gartmann, Holtkamp, Weissenberg, \& Li, 2005) (Chin \& Kontogiannis, 2004) permite el consumo de servicios en entornos en movilidad, de forma que los cambios de contexto se monitoricen y permitan reconfigurar el servicio para el contexto de ejecución actual. Las ontologías y el roaming de servicios se utilizan a menudo conjuntamente (Weißenberg, Gartmann, \& Voisard, 2006). Sin embargo, estos trabajos requieren de la utilización de un middleware de comunicaciones que soporte estos cambios de contexto.

Existen muchos middleware de comunicaciones, orientados mensajes, a llamadas a procedimientos remotos y también orientados a servicios (Ibrahim, 2009). Mientras que las plataformas middleware tradicionales típicamente emplean comunicaciones síncronas entre clientes y servidores, las nuevas soluciones estudiadas producen comunicaciones asíncronas y entre pares (P2P), llevan a arquitecturas de comunicaciones más débilmente acopladas y más adecuadas para los entornos de ejecución móviles (Morais \& Elias, 2010).

En este trabajo de tesis se define un proceso de resolución de componentes. Otros trabajos han abordado anteriormente este proceso, como en (Aguilera, Almeida, Orduña, López-de-Ipiña, \& de las Heras, 2010), basado en la ejecución continua de servicios en movilidad. Este proceso también se ha tratado desde el punto de vista de los sistemas autónomos (Kephart \& Chess, 2003), con aplicación también a servicios móviles en (Strassner, Meer, Foghlu, Leon, \& Donnelly, 2009).

Los trabajos relacionados con la delegación de servicios tratan los casos de la fragmentación de servicios, la provisión de modelos de coste para la decisión de la mejor estrategia de delegación, y la ejecución distribuida.

Relacionado con la fragmentación de servicios existen muchos trabajos que modelan un servicio como un proceso de negocio (business process) o como flujo de trabajo o workflow. De esta forma, se establecen técnicas para su descomposición en fragmentos más simples (Ramón Alcarria, Martín, Robles, \& Sánchez-Picot, 2016). Vanhatalo et al. (Vanhatalo, Völzer, \& Leymann, 2007) presenta una técnica para la descomposición de fragmentos que una entrada y una salida, llamados SESE (Single Entry Single Exit). Otros trabajos (Ramón Alcarria, Martín, Robles, \& Manso, 2017) proponen la 
identificación de componentes triconectados (Tarjan \& Valdes, 1980) para la descomposición de servicios. Esto permite la posibilidad de transformar un servicio expresado en un lenguaje de modelado como BPMN (Business Process Modeling Notation) en uno descrito mediante lenguaje ejecutable como BPEL (Business Process Execution Language). El uso de componentes triconectados se describe en profundidad en (Vanhatalo, Völzer, \& Koehler, 2008) y (Polyvyanyy, Vanhatalo, \& Völzer, 2011).

Relacionado con la propuesta de modelos de coste, en el trabajo de Nanda et al. (Nanda, Chandra, \& Sarkar, 2004) propone un modelo de coste para la distribución de fragmentos. Aunque estas técnicas solo aplican a estructurad simples de secuencia y división paralela. Los algoritmos presentados en este trabajo de tesis con más genéricos y se encuentran a un nivel de abstracción mayor.

La mayoría de tareas realizadas en el proceso de ejecución han sido discutidas previamente en otros trabajos. Existen muchas contribuciones relacionadas con delegación de tareas (Horváth \& Telek, 2015), descomposición de workflows (Jaradat, Dearle, \& Barker, 2014), matchmaking de servicios (Kritikos \& Plexousakis, 2014). Estas propuestas, sin embargo, están basadas en soluciones monolíticas implementadas generalmente en una plataforma virtualizada e implementada en un controlador (Bordel Sanchez, Alcarria Garrido, Sánchez-de-Rivera, \& Sánchez-Picot, 2016). Este componente recibe las invocaciones de servicio a través de una interfaz uniforme y resuelve la ejecución utilizando hardware virtual. Esta solución, sin embargo, no resuelva los problemas de un escenario real, donde dispositivos hardware heterogéneos y con interfaces propietarias están interconectados a través de enlaces nopermanentes y con altos retardos. En este contexto, una solución como la propuesta en este trabajo de tesis debería poder recolectar información sobre los dispositivos hardware y decidir en qué elementos particular se debe ejecutar cada acción (enviando la orden correspondiente, utilizando el mensaje o la interfaz adecuada).

\section{Conclusiones y relación con los objetivos expuestos en la Sección 2}

En esta sección se han estudiado distintas visiones a nivel de servicio y aplicación para las WSAN como la visión de Internet del Futuro, Internet de las Cosas, Cloud Computing, y el modelo SOA.

Para la correcta provisión de estos modelos, se considera fundamental describir a través de un leguaje de descripción de servicios o SDL el concepto de servicio utilizado en este trabajo y el conjunto de fases y procesos que atraviesa, es decir, su ciclo de vida.

Relacionado con el modelo de servicios este estudio nos permite contribuir al Objetivo \#4: Crear un modelo de servicios en el contexto de la arquitectura definida en el Objetivo \#1, que considere los requerimientos de las comunicaciones limitadas entre las redes inalámbricas de sensores/actuadores y una herramienta de soporte para el desarrollo de servicios basada en cloud.

Relacionado con el ciclo de vida de los servicios. Los estudios realizados nos permiten desarrollar el Objetivo \#5: Definir el ciclo de vida de los servicios 
considerando la ejecución en entornos distribuidos, la movilidad del servicio, tolerancia a largos retardos y las fallas en la infraestructura de comunicación.

En esta sección se describen también los trabajos sobre delegación de servicios, especialmente los que tratan los casos de la fragmentación de servicios, la provisión de modelos de coste para la decisión de la mejor estrategia de delegación, y la ejecución distribuida.

Estos trabajos ayudarán al desarrollo de una estrategia flexible y eficiente para la delegación y distribución servicios, considerando los requerimientos de las redes inalámbricas de sensores/actuadores desplegadas en ambientes hostiles o remotos (Objetivo \#7). Las contribuciones relacionadas con este objetivo se describen en las Secciones 6 y 7.

En la Sección 8 se propone una tecnología para gestionar la ejecución de servicios a bajo nivel a través de un proceso de recolección de características de los dispositivos, y una selección dinámica de los dispositivos aptos para ejecutar las invocaciones. Estas contribuciones vienen a resolver el Objetivo \#8 (definir una estrategia de ejecución de servicios sobre nodos en redes de sensores y actuadores con comunicaciones limitadas).

\subsection{Arquitecturas}

En esta sección se resumen y analizan las principales arquitecturas de servicios sobre redes de telecomunicación. Posteriormente, se realiza una revisión de la literatura relacionada con las arquitecturas de integración de las WSAN a Internet, mediante servicios basados en tecnologías cloud.

Existen varias propuestas de arquitecturas de servicios sobre redes de telecomunicación, que responden a la necesidad de diseño, creación, desarrollo, despliegue, ejecución y gestión eficiente de nuevos servicios basados en la cloud, según la demanda del mercado.

Las investigaciones iniciaron como parte de la evolución de las Intelligent Network (IN) para la implementación de valor añadido a los servicios, integrando las IN con los nuevos estándares de gestión de las telecomunicaciones, como el Telecommunications Management Network (TMN). A esta investigación se unió la iniciativa de Telecommunications Information Networking Architecture (TINA), para la gestión y control de servicios multimedia integrados, y también temas de implementación de middleware subyacente para entornos de procesamiento distribuido. Esto fue seguido por la promoción de Parlay y 3GPP Open Service Access (OSA) Application Programming Interfaces (APIs).

Con la ampliación del rol de Internet y la noción emergente de voz sobre IP (VoIP), fue inventado el Session Initiation Protocol (SIP), y nuevos servicios de comunicación multimedia más radicales implementaron conceptos inspirados por las Information 
Technologies (IT) y la programación web, como los SIP Servlets. Con la creciente importancia de las Next Generation Networks (NGNs) y la planificada evolución de la redes fijas y móviles, hacia una sola Core Network $(\mathrm{CN})$ basada en Internet Protocol (IP), denominada por la 3GPP como IP Multimedia Subsystem (IMS), que emerge como una combinación práctica de protocolos SIP y VoIP, para brindar servicios IP Multimedia a usuarios móviles basados en UMTS (Blum, Müller, Schreiner, \& Magedanz, 2013).

Los IMS están basados en los estándares de entidades tales como the 3rd generation partnership as Project (3GPP) CN, ETSI Telecommunications and Internet Converged Services and Protocols for Advanced Networking (TISPAN) CN, 3GPP2, etc. Como se muestra en la Figura 1, un acceso idependiente es la principal característica de la CN, la cual hace posible su adaptación para la implementación de Machine to Machine (M2M) communication, ya que existe una variedad de dispositivos con diferentes características que pueden ser conectados a la $\mathrm{CN}$. Adicionalmente, IMS tiene un conjunto de aplicaciones bien definidas por la Open Mobile Alliance (OMA).

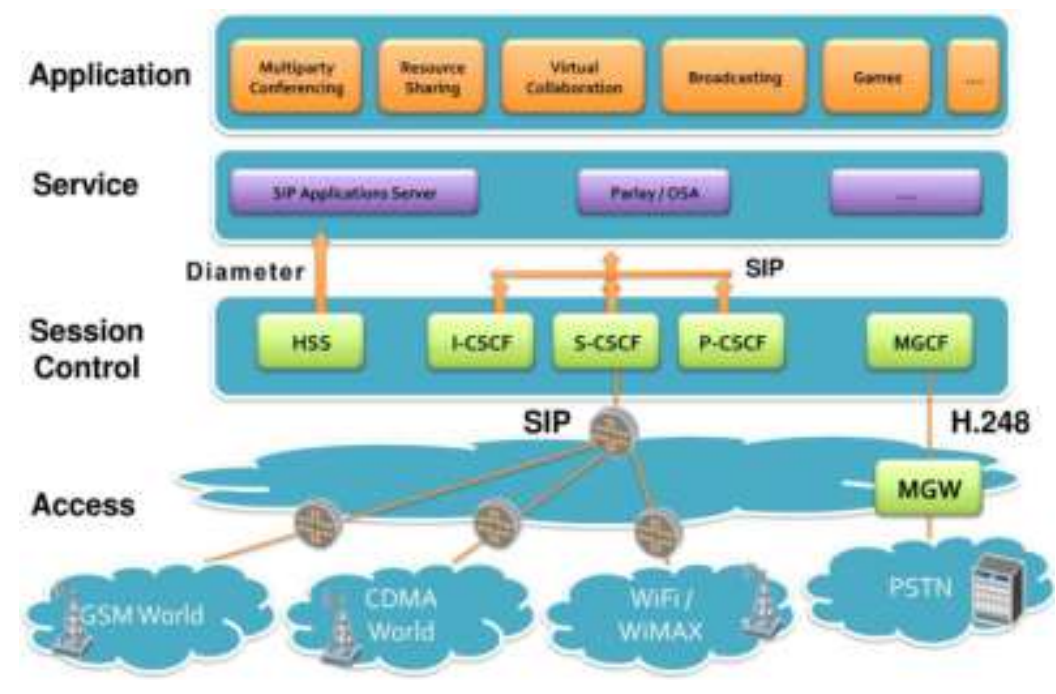

Figura 1. Arquitectura IMS.

La ETSI define la arquitectura específica para M2M Communication en (European Telecommunications Standards Institute, 2013, p. 1). La arquitectura simple de M2M se muestra en la Figura 2. La arquitectura funcional está diseñada para hacer uso de una red IP subyacente, que incluyen los servicios de redes IP provistos por 3GPP, TISPAN y sistemas compatibles 3GPP2. 


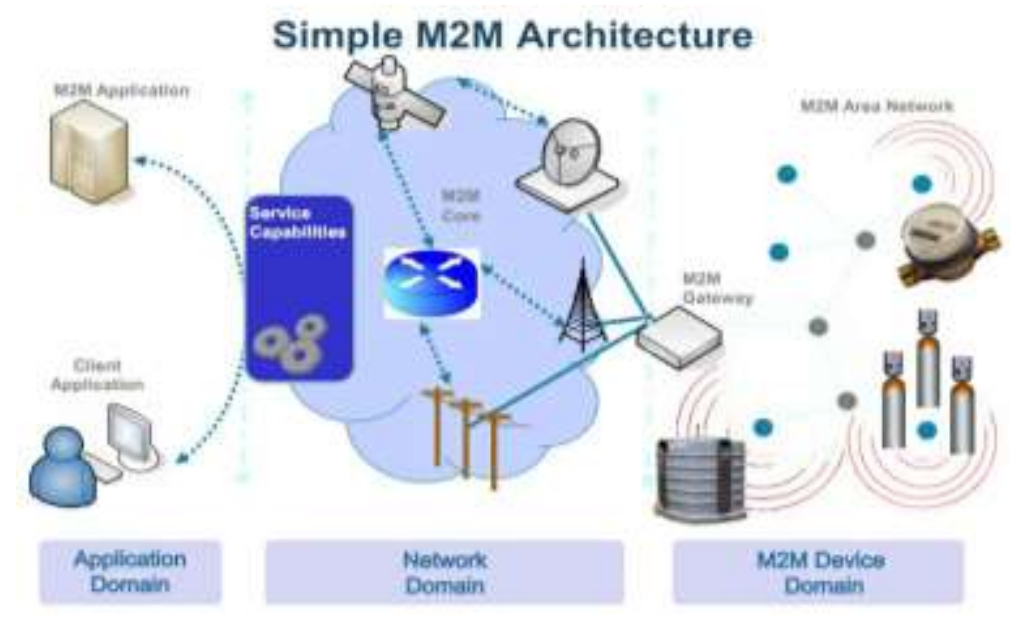

Figura 2. Arquitectura de alto nivel para M2M.

En (Villareal Padilla \& Jung Ho Kim, 2013), proponen una arquitectura de integración de IMS Core Network con Machine-to-Machine para proveer un framework de convergencia de aplicaciones, basadas en redes de dispositivos (sensors) que se conectan a la red de M2M de forma directa o a través de un gateway que proporciona servicios y capacidades de M2M communication. Este framework permite el intercambio de información entre los sensors y la red pública, además, provee mecanismos de comunicación entre ellos mismos. Esta arquitectura utiliza los conceptos y funcionalidades de los sistemas basado en IMS, adicionalmente brinda servicios y capacidades de M2M Communication. Esto hace posible la construcción de un framework convergente de aplicaciones.

EL 3GPP en (3GPP TR 23.888, 2012), propone una arquitectura llamada Machine Type Communication (MTC) para comunicación de datos (puede verse en la Figura 3), en la cual se incluye una o más entidades que no necesariamente necesitan interacción humana, como se muestra en la Figura 3. Las aplicaciones end-to-end, entre los UE (MTC Devices) utilizado para la MTC y la Aplicación MTC, utiliza los servicios provistos por el sistema 3GPP, y opcionalmente servicios provistos por un MTC Server. El sistema 3GPP provee servicios de transporte y comunicación (incluyendo servicios de portadora 3GPP, IMS y SMS). Las aplicaciones MTC pueden hacer uso de un MTC Server, para servicios de valor añadido, provisto por terceras partes (Service Provider).

Las arquitecturas de M2M y MTC, tienen elementos comunes, en términos de sus componentes y ambas son compatibles con IMS cores. 


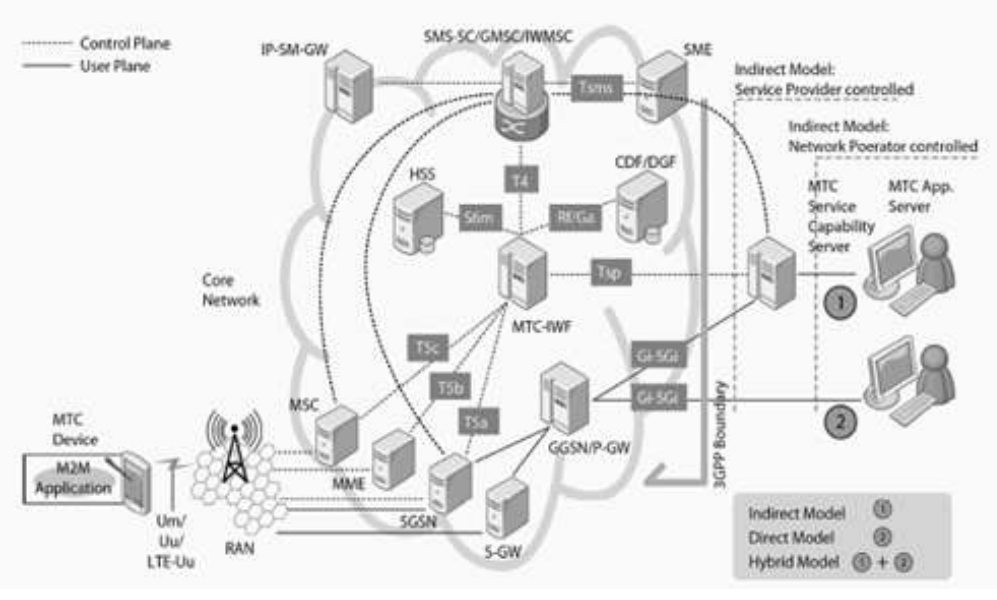

Figura 3. Arquitectura Machine Type Communication.

Por otro lado, la integración de las WSAN a la World Wide Web ha conllevado a la aparición de nuevas oportunidades de investigación. Existen diferentes trabajos relacionados con el desarrollo de arquitecturas para la integración de WSAN heterogéneas con otras redes basadas en All-IP, con el objetivo de acceder, recolectar y compartir datos. La integración de las redes de sensores a la cloud, ha sido investigada en (Kosanović \& Stojčev, 2011), (Kouche, 2012), (Li \& Yu, 2011) y (Mitton, Papavassiliou, Puliafito, \& Trivedi, 2012), los autores han propuesto a IP para conectar WSN a Internet, sin embargo, estos trabajos están enfocados principalmente en la factibilidad de servicios web basados en Simple Object Access Protocol (SOAP), en términos de energía y sobrecarga en el ancho de banda, adicionalmente, no presentan implementaciones reales.

En (Ting-hui et al., 2010), se propone una arquitectura de tres capas (sensores, par pasarela and super-par), basada en sistemas de integración P2P. En esta arquitectura las WSAN tienen como función recolectar información y el procesado simple de datos.

Una de las arquitecturas pioneras en la integración de las WSAN a la cloud fue SenseWeb (Kansal, Nath, Liu, \& Zhao, 2007), esta permitía compartir los datos de los sensores a través de la cloud. Sin embargo, su principal desventaja es que todas las decisiones son tomadas por un único punto central llamado coordinador, por lo tanto, si el coordinador falla, la red entera falla.

Esfuerzos similares están dirigidos al desarrollo de middleware para permitir el mapeo de dispositivos con recursos limitados a sistemas IT o Internet. Algunos de ellos se centran en el desarrollo de gateways que mapean las WSAN propietarias a sistemas IT, asumiendo que los gateways no son dispositivos con recursos limitados (Moeller \& Sleman, 2008), (Priyantha, Kansal, Goraczko, \& Zhao, 2008), sin embargo, estos sistemas no se consideran robustos, porque su principal desventaja es que tienen un simple punto de falla. Otro enfoque es el desarrollo de middleware para los propios dispositivos con recursos limitados (sensores y actuadores). En (Al-Yasiri \& Sunley, 2007) proponen utilizar SOAP para agregación de datos en cada sensor, así 
como también en (Wolff, Michaelis, Schmutzler, \& Wietfeld, 2007), se desarrolla un middleware que permite el despliegue de servicios en dispositivos con recursos limitados. Este enfoque tiene las limitaciones de los recursos de hardware de los dispositivos. La sobrecarga de software, no les permite brindar todas las funcionalidades, adicionalmente, asume dispositivos siempre conectados.

\section{Conclusiones y relación con los objetivos expuestos en la Sección 2}

En esta sección se han resumido y analizado las principales arquitecturas de servicios sobre redes de telecomunicación. La revisión de la literatura relacionada con las arquitecturas de integración de las WSAN en Internet nos permite concluir que los trabajos revisados asumen que las WSAN tienen conectividad permanente con otras redes, que permiten el acceso remoto a las mismas.

Sin embargo, estas soluciones no consideran entornos en donde las WSAN tienen comunicaciones limitadas, o con largos retardos. Las arquitecturas se caracterizan por capas de decisión principalmente alejadas de las WSAN. Analizan WSAN desde la perspectiva de la recolección de datos principalmente. Nuestra propuesta, permite ofrecer capacidades a las WSAN para enviar y recibir mensajes, además, incorpora facilidades de toma de decisiones en las entidades que están en contacto directo con los dispositivos finales. Adicionalmente, ofrece gestión de servicios tolerantes a entorno disruptivos y con largos retardos, basada en tecnologías DTN.

Este análisis nos permite contribuir al Objetivo \#1: Diseño de una de arquitectura de referencia para ejecución distribuida de servicios en redes inalámbricas de sensores y actuadores con infraestructura de comunicación limitada, desplegadas como sistemas monolíticos en zonas aisladas o desatendidas.

Finalmente, también se contribuye al Objetivo \#9: Desarrollo de una plataforma de simulación monolítica, liviana, configurable, flexible, altamente escalable y confiable, que proporcione soporte para simulaciones de ambientes distribuidos con comunicaciones limitadas, inalámbricas, disruptivas, y que permita la experimentación de las aplicaciones, prototipos y pruebas de conceptos, que validan las contribuciones en los distintos escenarios. 
3. EL ESTADO DEL ARTE 


\section{Planteamiento de la arquitectura general del sistema}

\subsection{Introducción}

El objetivo de este capítulo es el diseño del diseño de una de arquitectura de referencia y funcional para la ejecución distribuida de servicios sobre redes inalámbricas de sensores y actuadores con infraestructura de comunicación limitada, desplegadas en zonas aisladas o desatendidas (Objetivo \#1). Para erllo, se identifican los requisitos funcionales y no funcionales de la arquitectura, se proporciona un diseño de alto nivel de la (definiendo componentes, interfaces y protocolos), y finalmente se mapean los requisitos funcionales y las capa de la arquitectura (Objetivo \#2).

Esta arquitectura está conformada por tres macro segmentos principales: el primero lo constituyen las redes de sensores, cuyos nodos que disponen de recursos limitados, y se utilizan en escala reducida para realizar algunas funciones como recolección, procesamiento y transmisión de información. Luego encontramos el segmento intermedio de comunicación que es itinerante (en decir, que no tiene presencia continua), y que tiene dos funciones principales, la primera es el transporte de información entre el primer segmento y el tercero. La segunda función es proporcionar, mediante sus componentes, soporte para servicios móviles tomando el rol de host anfitriones temporales de los mismos. Finalmente hay un segmento de soporte a servicios y aplicaciones en la cloud, cuya función primordial es ofrecer facilidades para el desarrollo de servicios basados en las funcionalidades de las redes inalámbricas de sensores y actuadores distribuidas, y alojadas en zonas remotas.

En la Sección 4.2, yo analizo las características de las redes de sensores para identificar los requerimientos específicos, haciendo referencia especial en las comunicaciones externas. Posteriormente en la Sección 4.3, yo propongo el diseño de un modelo de alto nivel, que representa la arquitectura general del sistema bajo estudio. Adicionalmente en la Sección 4.4, se describen las capas, componentes, interfaces y protocolos del modelo propuesto. 
Basado en la arquitectura general definida en este capítulo, en el Capítulo 5, yo defino una interfaz de programación de aplicaciones, para el desarrollo de servicios, utilizando comunicación M2M y orientada a las redes inalámbricas de sensores y actuadores distribuidas, y con infraestructura de comunicación limitada.

\subsection{Descripción de la arquitectura propuesta}

En este apartado, presento una arquitectura extensible, escalable, integradora y flexible para WSAN distribuidas. El objetivo de esta arquitectura es entregar servicios y aplicaciones que puedan estar accesibles y compartidas con la comunidad de usuarios de Internet, mediante un framework de desarrollo de servicios basados en las capacidades de las WSAN distribuidas que no cuentan con facilidades de comunicaciones continuas de extremo a extremo. Los datos de las WSAN distribuidas, alojadas en sitios remotos y con condiciones de comunicaciones limitadas, pueden ser procesados, almacenados y analizados en la cloud, o de forma local en los componentes de la arquitectura con capacidades de desplazamiento, y que poseen facilidades para el contacto temporal y oportunista con los dispositivos de tipo cluster head, de esta forma se proporciona soporte para servicios móviles en el contexto de las WSAN distribuidas. La comunicación del framework de servicios en cloud, con las redes inalámbricas de sensores y actuadores, es itinerante, por lo tanto la arquitectura proporciona soporte para servicios persistentes ante los eventos de largos periodos de tiempo en espera, sin pérdida del estado de ejecución de los mismos. Adicionalmente, este modelo considera situaciones de pérdida o caída de los enlaces de comunicación, proporcionando desacoplamiento entre la ejecución de los servicios y el plano de comunicaciones entre los nodos participantes.

\subsection{Escenario de aplicación}

Como se puede observar en la Figura 4, las WSAN están ubicadas en sitios lejanos de infraestructuras de comunicación por cable o inalámbricas, por lo tanto sus comunicaciones con redes externas son muy limitadas. Las comunicaciones con redes externas son discontinuas, y están basadas en el paradigma de comunicaciones oportunistas.

Los datos recolectados por los dispositivos coordinadores (tipo cluster head) son entregados cuando aparezca en su rango de comunicación una entidad móvil que proporciones servicios de redes DTN, con la cual se pueda establecer un enlace para el intercambio de mensajes. Posteriormente, estas entidades utilizan la red de los operadores de telecomunicaciones para el transporte $y$ de control de las comunicaciones extremo a extremo entre ellos y el framework de servicios en la cloud. Los datos procedentes de las WSAN son almacenados, analizados y procesados por una capa que los gestiona, para ofrecerlos posteriormente como insumo de servicios en la cloud. 
En este contexto se han identificado principalmente dos tipos de servicios a saber: los servicios fijos y los servicios móviles. Los fijos tienen como principal característica, una conducta pasiva frente a los sensores y actuadores, es decir, los datos son los que se mueven, mientras los servicios siempre esperan a los datos para procesarlos, tomar decisiones y enviar mensajes a los actuadores (cundo sea el caso). Los servicios fijos están localizados de forma permanente en la cloud, es decir, son servicios cuya ejecución se lleva a cabo de forma completa en la cloud.

Por otro lado existen los servicios móviles cuya ubicación varía en función de su complejidad, recursos necesarios para su ejecución, disponibilidad de equipos con soporte en la zona de localización de las WSAN y las preferencias del usuario. Estos servicios pude estar la cloud o a bordo de las entidades móviles, en cuyo caso la ejecución se lleva a cabo de forma remota, esto permite una ejecución de servicios cerca al origen de los datos, proporcionando mayor eficiencia en la ejecución.

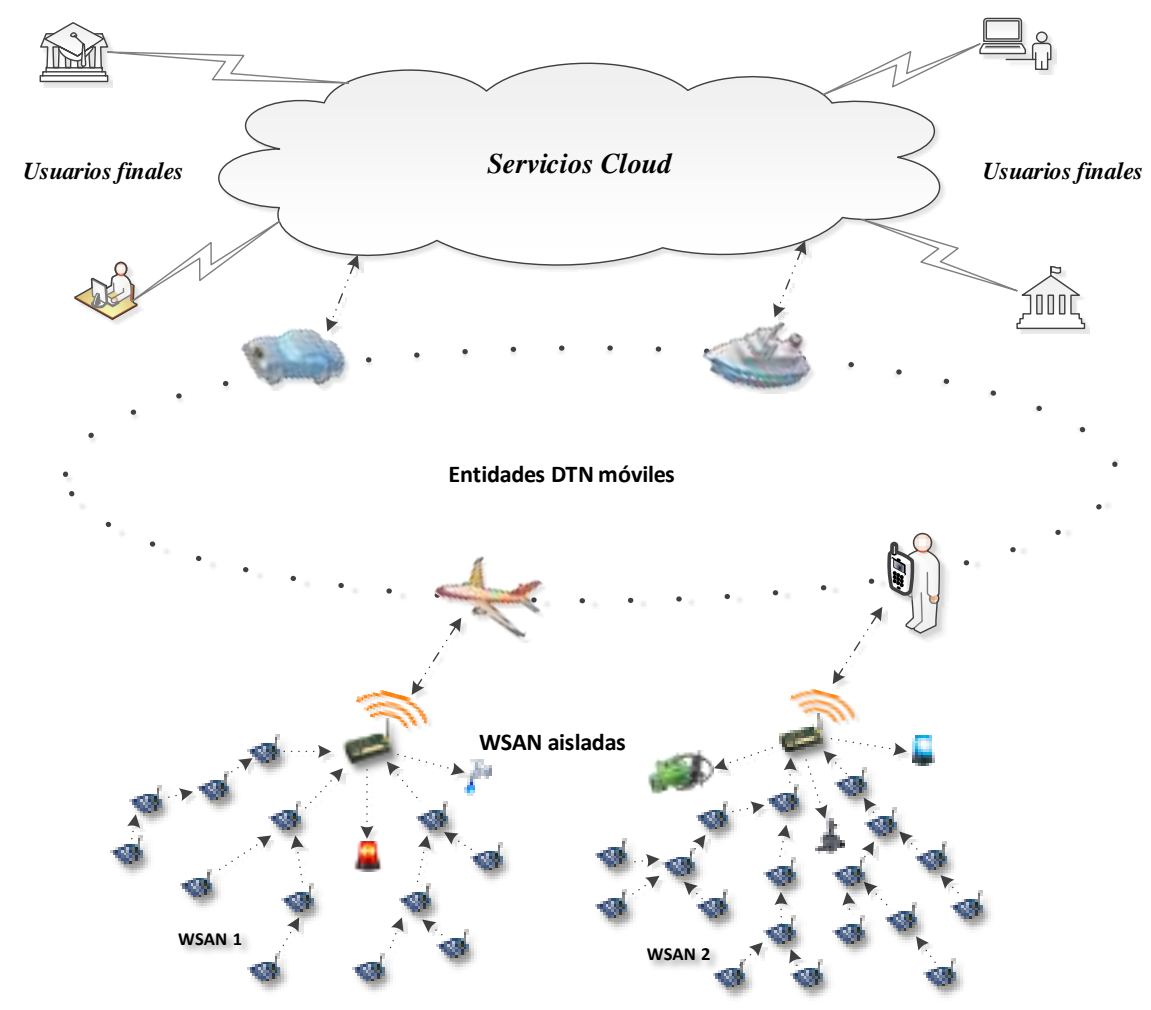

Figura 4. Escenario de motivación.

\subsection{Requisitos para una arquitectura de servicios sobre redes de sensores}

Como resultado del análisis previo he identificado los requerimientos funcionales y no funcionales que debe cubrir la arquitectura propuesta, los cuales se listan y definen a continuación. 


\subsubsection{No Funcionales}

El diseño de la arquitectura propuesta se debe caracterizar por los siguientes atributos de calidad:

- RnF1. Extensible, compatible, flexible y escalable: debe contemplar añadir nuevos componentes funcionales en cualquier nivel. Permitir la integración de múltiples tecnologías, y debe ser adaptativa en escenarios heterogéneos. Adicionalmente, debe soportar la adición de nuevos dispositivos y servicios, sin degradar el rendimiento de los existentes.

- RnF2. Enfoque horizontal: se refiere a un recubrimiento de facilidades abstractas de alto nivel, donde todas las aplicaciones comparten la infraestructura, el contexto y componentes de red, permitiendo el desacoplamiento vertical entre las aplicaciones y las WSAN.

- RnF3. Accesibilidad global: el repositorio de los servicios y aplicaciones desarrollados deben estar siempre accesibles desde cualquier dispositivo con acceso a Internet.

- RnF4. Soporte de interconexiones discontinuas con las WSAN: debe permitir interacciones intermitentes a intervalos regulares o irregulares, y con extensos aplazamientos.

\subsubsection{Funcionales}

Los componentes de la arquitectura deben cumplir con las siguientes funcionalidades:

- RF1. Los dispositivos coordinadores (cluster head) de las WSAN deben contar con capacidades de comunicación entre los sensores/actuadores y las redes externas, además de actuar como sumidero de datos de la red de sensores subyacente.

- RF2. Proporcionar un medio para el transporte bidireccional de información entre las WSAN con comunicaciones limitadas y la cloud (Internet).

- RF3. Provisión de mecanismos de comunicación entre los dispositivos coordinadores que controlan cada WSAN (mula entre cluster head).

- RF4. Soporte de comunicación de extremo-a-extremo entre los dispositivos coordinadores y los servidores de gestión de servicios y aplicaciones.

- RF5. Gestión del ciclo de vida (creación, personalización, publicación, búsqueda y ejecución) de servicios tolerantes a retardos y desconexiones.

- RF6. Capacidad de delegación de la ejecución de servicios a entidades en contacto directo con los sensores y actuadores.

- RF7. Interacción transparente entre la plataforma de gestión de servicios y el framework de desarrollo de servicios en cloud, para la publicación de servicios que han sido creados considerando los recursos disponibles en las WSAN. 


\subsection{Diseño de la arquitectura de alto nivel}

Este apartado muestra el modelo de la arquitectura propuesta. Primero se expone la arquitectura de alto nivel, que ofrece una visión general y abstracta del modelo. Constituye el marco de referencia de la arquitectura, y puede interpretarse desde el plano físico y desde el plano funcional, que se describían en las secciones 4.5.2 y 4.5.3 respectivamente. Esta interpretación dual de la arquitectura de alto nivel, permite asimilar mejor la forma en que satisfacen los requisitos definidos en la sección 4.4.

\subsubsection{Visión abstracta}

La vista general de la arquitectura, que puede observar en la Figura 5, se compone de las siguientes capas: aplicación, servicio, DTN y la capa de dispositivos.

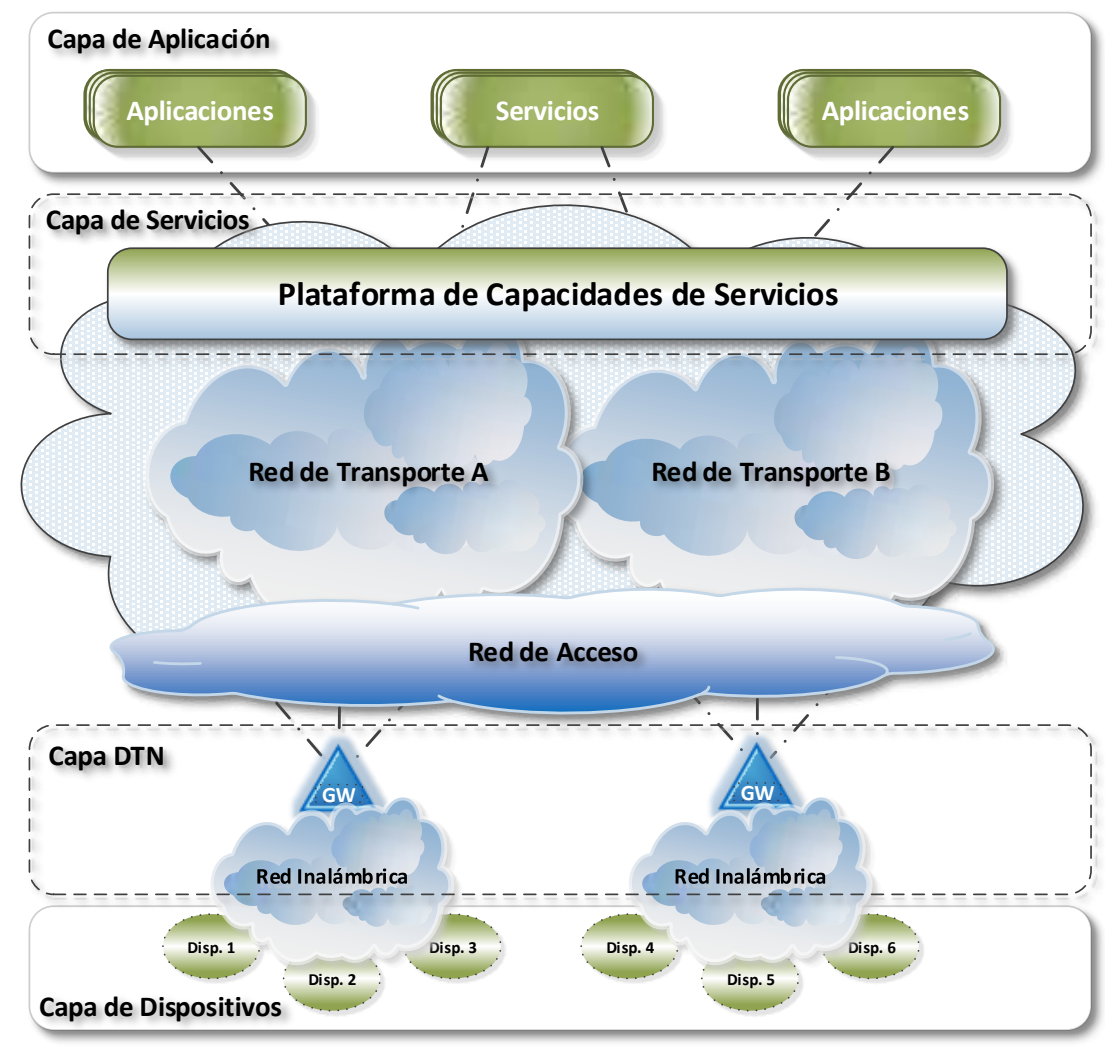

Figura 5. Arquitectura general.

La capa de aplicación está compuesta por el conjunto de aplicaciones y servicios, publicados y disponibles para los usuarios finales. Estas aplicaciones y servicios están soportados por la Plataforma de Capacidades de Servicio, situada en la capa de servicio. La Plataforma de Capacidades de Servicio está formada por un framework común de gestión de servicios, que permite el desarrollo de nuevos servicios basados en la disponibilidad de los recursos de las WSAN. También incluye capacidades de servicio para comunicaciones M2M y gestión de servicios móviles.

En el nivel inmediatamente inferior a la capa de servicio, se encuentra la red del proveedor de servicios (red de acceso y transporte), que contiene los servicios y las 
funciones de control de la red (control de sesión, administración, autenticación de suscriptores, autorización de servicio y localización). Este provee interconexión con los servicios de redes IP (3GPP/IMS, TISPAN y sistemas compatibles 3GPP2), adicionalmente en la red de acceso de los operadores existen varias tecnologías disponibles (GPRS, GSM, WIMAX, LTE y otras).

La capa DTN ofrece un conjunto de funcionalidades para soportar comunicaciones con largos retardos y desconexiones de red. Consiste de una o varias entidades móviles (denominadas "Gateway" -GW- en las Figuras 5 y 6) que permiten comunicaciones entre las WSAN y las redes externas, basadas en un enfoque oportunista.

En la parte inferior se encuentra la capa de dispositivos, compuesta por las redes inalámbricas de sensores y actuadores. En este esquema los dispositivos representan a los respectivos cluster head de cada WSAN.

\subsubsection{Descripción física}

La arquitectura de alto nivel puede verse como un conjunto de capas apiladas, y en cada una de ellas existen un conjunto de elementos que la integran y que están interconectados. En la Figura 6, se muestra el plano físico, considerando las capas de la arquitectura de alto nivel presentadas en la sección anterior.

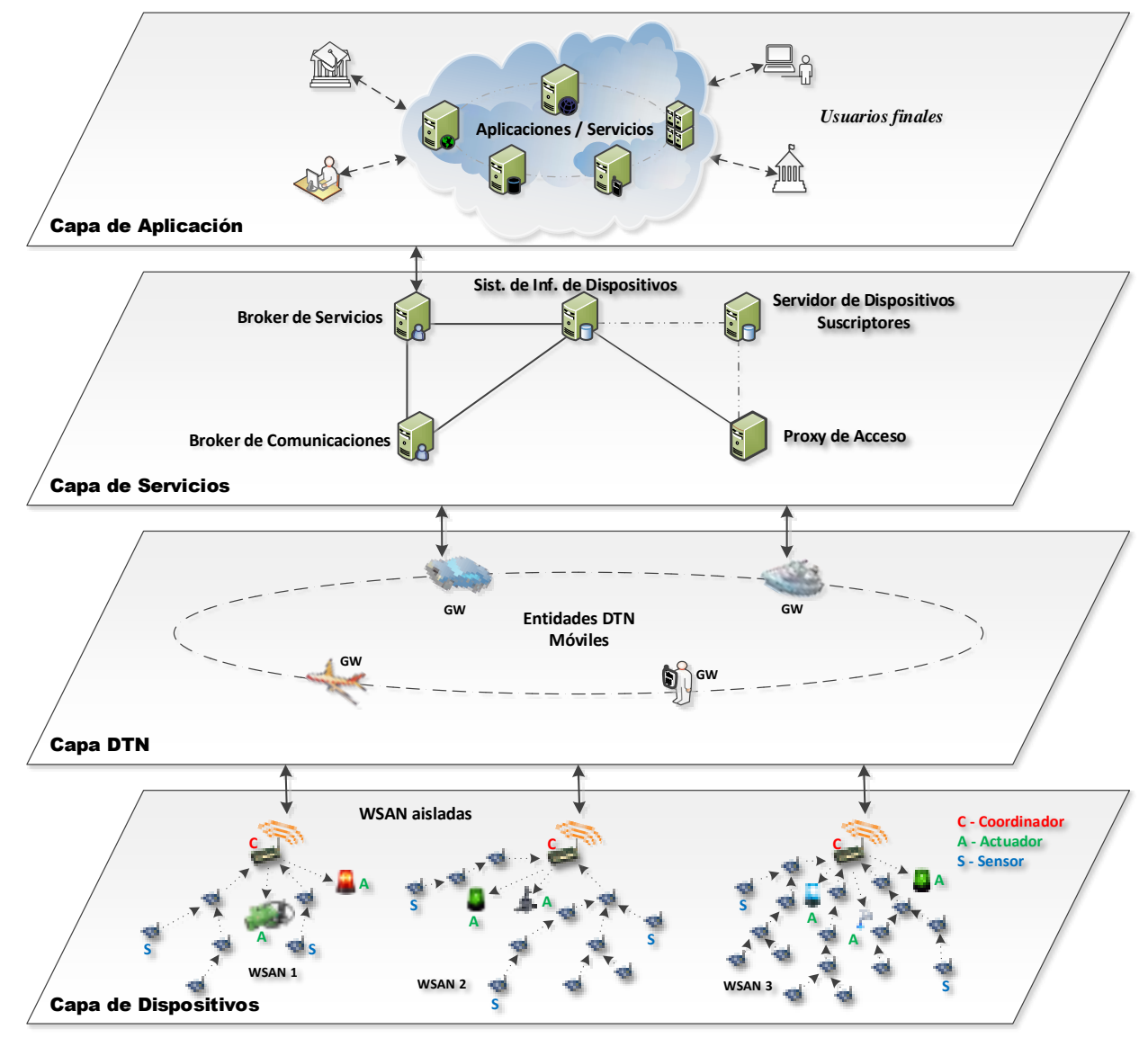

Figura 6. Plano físico de la arquitectura. 
Capa de dispositivos: está conformada por las WSAN. Los cluster head deben tener la capacidad de comunicación con los sensores y actuadores. Adicionalmente, cuentan con capacidades de comunicación a nivel de DTN, para las comunicaciones con las entidades DTN móviles. También proporcionan funcionalidades de gateway, entre las redes IP y las tecnologías de redes de sensores 802.15.4 (ZigBee/6LoWPAN), Bluetooth, Bluetooth Low Energy, 802.11 (Wi-Fi) y otras.

Capa DTN: está formada por una o varias entidades móviles (identificadas como “Gateway" en las Figuras 5 y 6, por su carácter de puerta de acceso a la Intenet global) con capacidades DTN (Entidades DTN Móviles). Las capacidades de comunicación DTN se utilizan para tener acceso a la capa de servicios, a través del Proxy de Acceso (PA). Las entidades DTN móviles ofrecen un mecanismo de transporte bidireccional entre las WSAN y la red de acceso del Proveedor de Servicios de Red (PSR). Pueden soportar capacidades de servicios para comunicación entre los cluster head de las WSAN (capacidades de servicios M2M), y comunicaciones entre WSAN y la Plataforma de Capacidades de Servicio. Proporcionan soporte para ejecución de servicios móviles y toma de decisiones.

Capa de servicios: está formada por servicios de acceso y transporte del Proveedor de Servicios de Red, y por componentes de gestión de servicios tolerantes a retardos extensos. El Proxy de Acceso es el responsable de controlar el establecimiento de comunicación entre las Entidades DTN Móviles y los Brokers de servicios, para esto cuenta capacidades DTN. Para el establecimiento de sesiones, el Proxy de Acceso utiliza la información de perfiles de usuarios almacenada en el Servidor de Dispositivos Suscriptores (SDS), y basado en esta información, controla el acceso al Sistema de Información de Dispositivos (SID), que gestiona la información de los dispositivos (características, estados, accesos, servicios, localización, etc.).

El Broker de Comunicaciones (BC) y Broker de Servicios (BS), tienen como funcionalidad principal, permitir compartir los recursos de las WSAN, además de proveer una interfaz transparente con la cloud. Los BS ofrecen las capacidades de habilitación y registro de aplicaciones, administración remota de dispositivo, seguridad (servicio de bootstrap, autenticación mutua, acuerdo de key y verificación de integridad). Los BC ofrecen capacidades de comunicaciones genéricas (establecimiento de sesión de transporte, aseguramiento de la entrega, reporte de errores), selección del PSR y otras.

Capa de aplicación: define una infraestructura en cloud, formada por un conjunto de servidores (web, movilidad, aplicaciones, base de datos, gestión de usuarios, seguridad y otros) que proporcionan el soporte para la publicación de aplicaciones y servicios, enriquecidos con las prestaciones de la computación en cloud.

\subsubsection{Descripción funcional}

La arquitectura definida en la sección 4.5.1, puede entenderse como un conjunto de componentes funcionales relacionados entre sí. En la Figura 7, se muestra la 
descomposición de los principales componentes funcionales de la arquitectura propuesta.

En la capa de dispositivos se muestra la estructura de un nodo cluster head. El gestor de comunicaciones es responsable de establecer comunicaciones internas (WSAN) y externas (entidades DTN móviles), para esto utiliza un doble stack de protocolos. Utiliza las comunicaciones internas para recopilar la información de la red de sensores subyacente, y almacenarlos mediante el Gestor de Datos (RF1). Implementa las funciones de proxy entre la WSAN y las redes externas, mediante el WSAN Gateway. Las redes externas son de tipo DTN, por lo que utiliza las funcionalidades del módulo de DTN, para establecer esta comunicación.

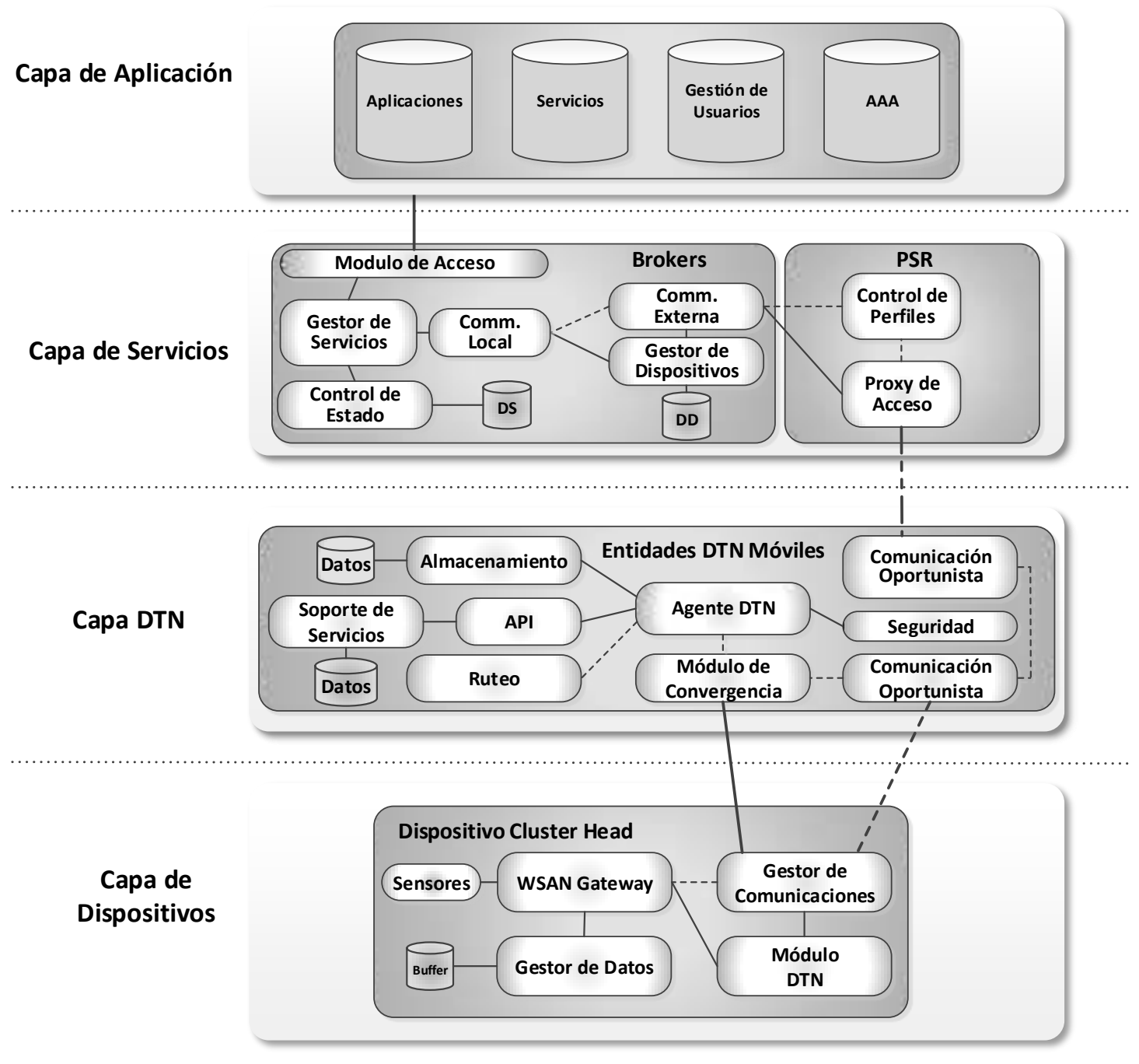

Figura 7. Arquitectura funcional.

Las Entidades DTN Móviles, están estructuradas como se puede observar en la capa DTN de la arquitectura funcional. Las entidades DTN tienen como objetivo actuar como medio de comunicación entre las WSAN y la capa de servicios. Utilizan un 
enfoque de comunicación oportunista. El Agente DTN es el núcleo de las entidades DTN móviles, su función es coordinar la ejecución de tareas, que se necesiten realizar, en función de los eventos internos y externos a la entidad. El módulo de Comunicación Oportunista determina cuando existe un vecino con el que se puede comunicar, identifica la capa de convergencia disponible en el vecino y determina si la tiene disponible en el Módulo de Convergencia, luego verifica si tiene datos para enviar (según las políticas del Módulo de Ruteo), o si es una solicitud de recepción. Las DTN Mobile Entities son utilizadas para comunicar las WSAN, con la red del PSR, que posteriormente permite alcanzar la capa de servicios. El mecanismo anteriormente descrito funciona de la misma forma en ambos sentidos de la comunicación (RF2).

El módulo de Soporte de Servicios, posibilita la ejecución de servicios delegados en la capa DTN (RF6). Este componente funcional puede ofrecer capacidades de servicios (registro de servicios, ruteo y otros) que permiten la comunicación a nivel de servicios, entre los cluster head de las WSAN (RF3), haciendo posible la ejecución de lógica de servicios en componentes que están en contacto directo con el origen de los datos.

La capa de servicios permite la gestión de servicios, ofrece funcionalidades principales como: creación, personalización, publicación, búsqueda y ejecución de servicios tolerantes a fallos y retardos en la red. Esta capa contiene toda la lógica de negocio. Ofrece capacidades de delegación de servicios y toma de decisiones en la capa DTN. El Gestor de Servicios controla el ciclo de vida de los servicios, mediante el módulo de Control de Estado almacena toda la información y el estado de los servicios (DS). Las comunicaciones internas las realiza el Comunicador Local, que es el responsable del flujo de datos y de control, entre el Gestor de Servicios y el Gestor de Dispositivos (información local). Adicionalmente, gestiona también las comunicaciones con el módulo de Comunicación Externa (información no local), situado en el SID. El módulo de Comunicación Externa es encargado de las comunicaciones con el módulo Proxy de Acceso, y con el módulo Control de Perfiles localizado en el SDS. El Proxy de Acceso proporciona capacidades DTN, por lo que puede recibir solicitudes de comunicación con dispositivos que actualmente pueden no estar disponibles, y que serán realizadas cuando exista la oportunidad. Este grupo de funcionalidades, permite dar soporte a servicios tolerantes a fallos y retardos en la red (RF5).

El Módulo de Acceso, proporciona un API de comunicación con la capa de servicios. Este API permite el acceso a la plataforma de gestión de servicios, que posibilita compartir los recursos de las WSAN. Adicionalmente ofrece una interfaz para la publicación de los servicios en la infraestructura cloud (RF7).

\subsection{Conclusiones}

El objetivo de este capítulo es el diseño de una de arquitectura de referencia y funcional para la ejecución distribuida de servicios sobre redes inalámbricas de sensores y actuadores con infraestructura de comunicación limitada, desplegadas en 
zonas aisladas o desatendidas. Para ello se definen los tipos de dispositivo existentes en el planeamiento de este trabajo, como los dispositivos coordinadores o cluster head, las entidades DTN móviles y las redes WSAN aisladas.

Seguidamente la arquitectura de la solución es propuesta, a través de una descripción física (arquitectura compuesta por elementos y otros dispositivos clasificados por capas), y una descripción funcional (entendiendo la arquitectura como un conjunto de componentes funcionales relacionados entre sí).

Como conclusiones particulares del capítulo cabe destacar la necesidad de abordar de manera más detallada la lógica de negocio relacionada con la delegación de servicios, la gestión de dispositivos y el control de perfiles. Contribuciones relacionadas con estos elementos funcionales son descritas en detalle en las Secciones 6 y 7. 


\section{Modelo de servicio y gestión del ciclo de vida}

\subsection{Introducción}

En este capítulo se define un modelo de servicios en el contexto de la arquitectura definida en el Capítulo 4, que considere los requerimientos de las comunicaciones limitadas entre las redes inalámbricas de sensores/actuadores y una herramienta de soporte para el desarrollo de servicios basada en cloud (Objetivo \#3). Además, defino la capa de gestión de servicios distribuidos tolerantes a retardo que permita la creación, distribución y ejecuación eficiente de los mismos empleando infraestructuras de WSAN aisladas (Objetivo \#4), según el modelo de referencia general de la Sección 4.3. En esta capa se concibe un el ciclo de vida de los servicios considerando su ejecución en entornos distribuidos, la movilidad del servicio, tolerancia a largos retardos y las fallas en la infraestructura de comunicación (Objetivo \#5).

La capa de gestión está formada por un conjunto de procesos que permiten la creación, publicación, modificación, personalización y ejecuación de servicios. Esta capa está basada en tecnologías DTN, de forma que los servicios no siempre cuentan con comunicación de extremo-extremo, y los retardos pueden ser muy extensos.

En esta sección se conceptualizan los diferentes procesos involucrados en la gestión de un servicio a lo largo de su ciclo de vida, particulrizado para el caso de su ejecuación sobre redesd e sensores y actuadores aisladas y capcidaes de comunicación limitadas. Además, para cada proceso, se analizan y describen los pasos que se deben cumplirse a fin de lograr una correcta gestión del servicio.

\subsection{Modelo de servicio}

En esta sección se define el modelo servicios tolerantes a fallos y retardos en la red. Se describen los elementos componentes del servicio, y las fases en las que un servicio puede encontrarse dentro del su ciclo de vida. 
Un servicio es definido, según OASIS ("Reference Model for Service Oriented Architecture 1.0," 2006), como "un mecanismo para permitir el acceso a una o más capacidades, donde el acceso se presta mediante una interfaz prescrita, y se trabaja de acuerdo con las políticas y limitaciones, como se especifican en la descripción del servicio". Basado en la referencia anterior definimos nuestro modelo de servicio como:

Un servicio tolerante es una pieza de software atómica, móvil y ejecutable, que realiza varias transiciones de fase durante su ciclo de vida, y su estado es persistente ante retardos, permite el acceso a capacidades de recursos heterogéneos mediante una interfaz predefinida, y es contenedor de lógica de negocio.

Según el entorno descrito en la Sección 4.3, es necesario que los servicios tolerantes cumplan con requisitos adicionales, que se definen a continuación:

Delegación: se define como la propiedad de un servicio que permite moverlo con destino a otro anfitrión, en el cual se llevará a cabo la gestión de su ejecución. En el contexto de la arquitectura definida en la Sección 4.5.2, los anfitriones sujetos de delegación de servicios son las Entidades DTN Móviles.

Asincronismo: los servicios deben estar desacoplados temporal y espacialmente, debido al comportamiento indeterminista de la red que permite acceder a los recursos y capacidades de las WSAN.

Estado: un servicio tiene siempre definido un estado actual dentro de su ciclo de vida. El estado de un servicio puede estar sujeto a transiciones, producto de uno o varios eventos durante su existencia. Los estados pueden ser: inicio, ejecución, parado, esperando, suspendido y finalizado.

Atomicidad: esta propiedad se define como la indivisibilidad del servicio, es decir, los servicios no pueden ser fragmentados. Esto conlleva a que los servicios deben seguir el paradigma de orquestación.

Los requisitos de asincronismo, estado y atomicidad, son importantes para la definición de servicios tolerantes, pero el requisito de delegación también es muy importante, además es esencial para nuestro modelo de servicios, porque ofrece la ventaja de poder ejecutar los servicios en zonas más cercanas al origen de los datos, permitiendo optimizar las oportunidades de comunicación con las WSAN, ya que éstas cuentan con comunicaciones muy limitadas. Desde el punto de vista de la red, los servicios se resuelven con enfoque de comunicación per-to-per, sin provocar sobrecargas en la infraestructura de comunicación, desde la capa de dispositivos hasta la capa de servicios, que se traduce en una eficaz y eficiente ejecución del servicio.

\subsubsection{Elementos de un servicio}

En esta sección se esquematiza mediante UML, el modelo de servicios tolerantes, y se identifican cada una de las piezas que lo conforman. También se definen los conceptos de plantilla, componente, restricción, capacidad, conectores, lógica, interfaz de usuario (IU), código fuente y metadatos del servicio. 
Los servicios tolerantes, están integrados por un conjunto de elementos y relaciones, cuyo modelo en UML se muestra en la Figura 8.

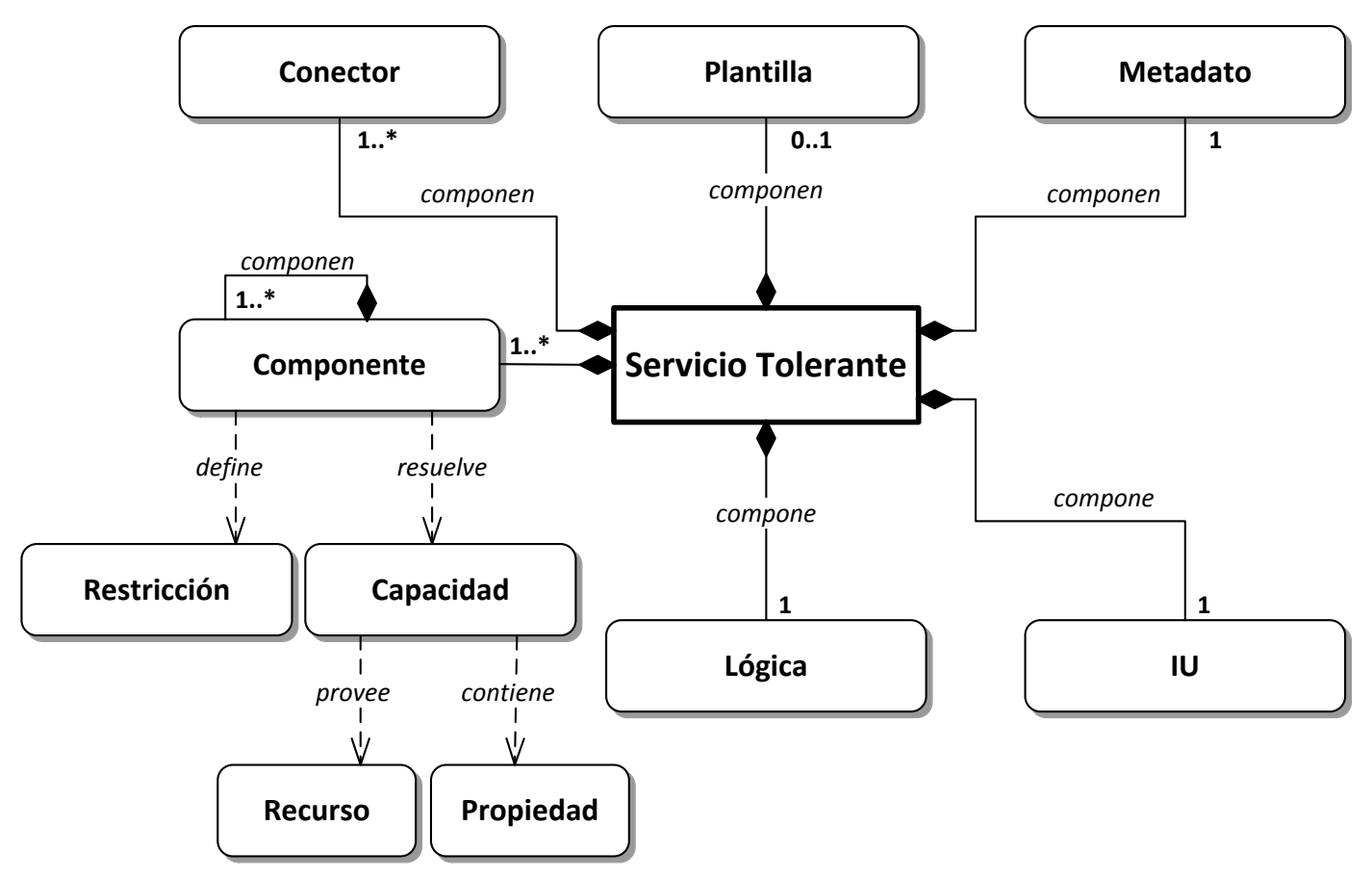

Figura 8. Modelo UML de servicios tolerantes.

Una plantilla se define como un pre-servicio, que luego de un proceso de adaptación pueden convertirse en un servicio. Las plantillas son mutables, no son componentes ejecutables hasta que se transforman en servicio. La utilización de plantillas permite separar la creación de servicios en dos partes, creación y adaptación. Otra ventaja es la reutilización de las plantillas, las cuales tienen implementado en el código un comportamiento genérico, que admite mecanismos de adaptación para convertirlos en servicios a medida.

Los servicios y plantillas, están integrados por diferente software de aplicaciones como: workflows, GUIs, librerías, servicios de base de datos, mensajería y otros, que interactúan con el middleware de comunicaciones, servidores, DBMS, motores de workflows y sistemas de mensajería.

Los Componentes de un servicio, son una visión abstracta de los recursos físicos reales, que pueden ser implementados usando recursos de software y hardware. Los componentes permiten desacoplar la representación lógica de la funcionalidad, de su implementación.

Las Capacidades de un servicio, son las implementaciones de los componentes, que ofrecen utilidad práctica. Un componente y una capacidad son compatibles si coinciden a nivel funcional. Las capacidades proporcionan acceso a la funcionalidad que representan, mientras ocultan las características del recurso físico subyacente, que 
es que realmente realiza las acciones (por ejemplo: actuadores y sensores). Las capacidades incluyen metadatos que definen sus propiedades.

Las Restricciones de componente, son propiedades no funcionales incluidas por el usuario en el momento de la creación del servicio. Estas permiten seleccionar las capacidades más adecuadas de acuerdo al creador del servicio.

Los Conectores, son las entidades responsables de coordinar el intercambio de mensajes (flujo de datos y control) entre los componentes. Estos conectores pueden visualizarse como enlaces de sincronización entre componentes, como en el enfoque Reo (Arbab, 2004), o puertas lógicas para el intercambio de eventos de control, como en los trabajos de Aalst et al. (2003).

La Interfaz de usuario, es un elemento que contiene la información relacionada con la interfaz, incluye información referente a las especificaciones del renderizado.

La Lógica del servicio, es el código ejecutable que implementa las relaciones entre los componentes del servicio, permite definir los patrones de comportamiento en función de los eventos.

Los Metadatos del servicio, son un conjunto de datos que contienen toda la información sobre las propiedades del servicio. Estos metadatos pueden ser añadidos en la creación, cuando se cargan en el repositorio o cuando una plantilla de servicio es instanciada. Cada servicio tolerante debe contener los siguientes metadatos:

- Creador del servicio: es el usuario dueño o creador del servicio.

- Fecha de creación: fecha en que fue construido el servicio.

- Fecha de publicación: fecha en que es puesto a disposición de los usuarios.

- UUID (universally unique identifier): identificador único universal.

- Tipo de servicio: determina si el servicio puede ser delegado o no.

- Descripción del servicio: describe qué hace el servicio.

- Descripción funcional: describe cómo se ejecuta el servicio.

- Configurable: determina si el servicio puede ser parametrizado.

- Métodos (entradas/salidas): define los métodos utilizados, sus entradas y salidas.

- Pre-condiciones: define los requisitos previos a la ejecución.

- Pos-condiciones: definen los requisitos posteriores a la ejecución.

- Fase: determina la etapa actual del servicio, dentro las fases del ciclo de vida.

- Estado: situación actual de un servicio dentro la fase del ciclo de vida en el que se encuentra actualmente.

- Lógica: código ejecutable que implementa las relaciones entre los componentes del servicio.

- Localización de ejecución: determina si la ejecución es local o remota.

- Objetos de delegación: en el caso de ser una ejecución remota, establece a quienes puede ser delegado el servicio. 


\subsection{Ciclo de vida de un servicio}

En esta sección se define el ciclo de vida de un servicio tolerante, como un conjunto de procesos interrelacionados, definidos utilizando SPEM 2.0, el cual es un lenguaje de modelado utilizado en ingeniería de software para definir metodologías y procesos.

SPEM (OMG, 2008). SPEM (Software Process Engineering Meta-Model) es una especificación de la OMG (Object Management Group) utilizada para definir software y sistemas de desarrollo de procesos, así como sus componentes. Su principal objetivo es cubrir un amplio rango de desarrollo de métodos y procesos en diferentes estilos, niveles de formalismos y modelos de ciclo de vida.

Los elementos SPEM utilizados en esta sección se describen en la Tabla 2:

Tabla 2: Descripción de los elementos de SPEM.

\begin{tabular}{|l} 
Proceso \\
An proceso es un componente divisible, ejecuta un trabajo concreto que \\
representa una relación entre instancias de actividades y los roles de las \\
instancias.
\end{tabular}

Los trabajos sobre gestión del conocimiento, de Nonaka y Takeuchi (1995), y los trabajos de Rus y Lindvall (2002), consideran que los bienes de conocimiento deben ser gestionados de acuerdo a su ciclo de vida. Para la gestión de ciclo de vida, un servicio tolerante puede enfocarse como un conjunto de elementos relacionados de acuerdo al conocimiento transferido, a través del lenguaje de lógica de servicio, utilizado por el creador del servicio. Por lo tanto, el conocimiento de los servicios tolerantes es distribuido, utilizado, adaptado y reutilizado en diferentes procesos del ciclo de vida. 


\subsubsection{Definición de actores y ámbitos}

En esta sección de definen los actores y ámbitos que están implicados dentro del ciclo de vida de los servicios tolerantes.

EL ciclo de vida de los servicios tolerantes se concibe en un entorno que incluye la participación de varios roles, los cuales están implicados en los diferentes procesos del ciclo de vida. En este sentido se definen los siguientes actores y ámbitos:

Usuario: es quien desarrolla servicios y hace que estén disponibles a otros usuarios o consumidores.

Consumidores: son los clientes finales de los servicios, su objetivo es consumir los servicios y aplicaciones provistas por el usuario del framework de desarrollo $\mathrm{u}$ otros interesados.

Dominio de aplicación: es el contexto en el cual los usuarios desarrollan sus servicios. Determina la naturaleza de los servicios creados y el alcance del framework de desarrollo.

Experto de dominio: es un usuario final que es elegido por sus conocimientos en el dominio de la aplicación, requerimientos y los objetivos de creación. La selección del experto de dominio, puede ser del grupo de usuarios avanzados, ya que tendrá también el conocimiento de cómo un método de creación encajaría en el dominio de la aplicación.

Proveedores de framework: son un grupo de usuarios o una entidad (administradores, analistas, desarrolladores), que son expertos es desarrollo de infraestructuras de software, con la asistencia del experto de dominio para un framework de provisión de servicios, que incluye un conjunto de herramientas adaptadas a las posibilidades de creación del usuario.

Especialista de framework: es un experto de framework, que conoce perfectamente las capacidades y limitaciones de un framework de creación de servicios. El especialista de framework puede desempeñar funciones de capacitación, asistencia al usuario en tiempo de creación, etc.

\subsubsection{Ciclo de vida de servicios tolerantes}

En esta sección se define el modelo del ciclo de vida de los servicios tolerantes. Se definen los procesos involucrados, y se describen las diferentes rutas que un servicio puede seguir, desde su fase inicial hasta lograr su objetivo.

Los servicios tolerantes pueden ser creados parcial o totalmente por los usuarios. Un servicio tolerante es creado, clasificado, publicado, buscado, adaptado, ejecutado y verificado. Puede ofrecer o no, una interfaz gráfica que permite acceder a una funcionalidad, e incluir de un conjunto de componentes que puede ser ejecutados.

En el diagrama de la Figura 9, se muestran cada una de los procesos por los que atraviesa un servicio durante su ciclo de vida. 


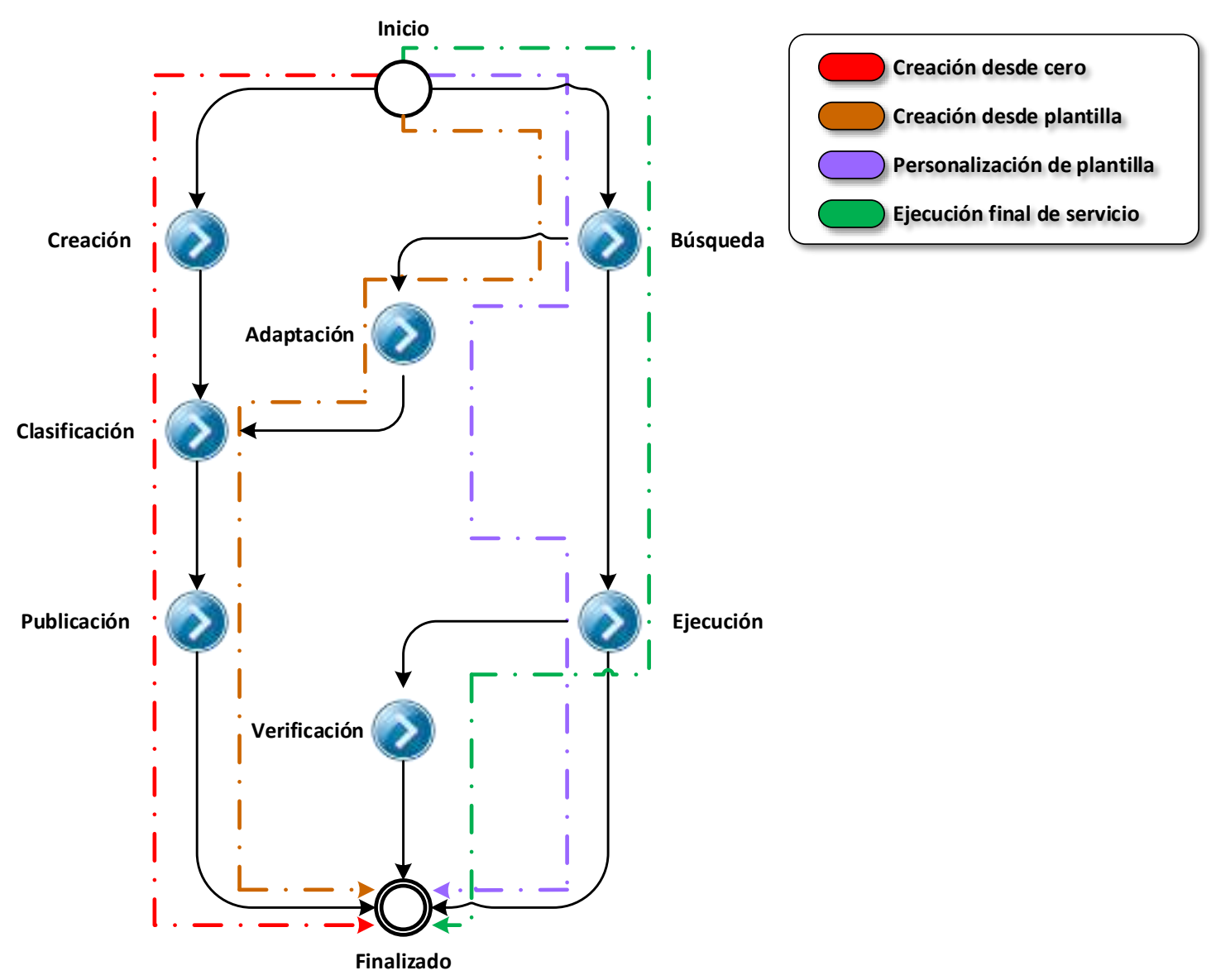

Figura 9. Procesos del ciclo de vida de un servicio tolerante.

Los servicios inician su vida en el proceso de creación, en el cual los usuarios construyen sus servicios utilizando alguna herramienta proporcionada por el framework de servicios. La descripción de servicio suministrada por el usuario, es utilizada en el proceso de clasificación para generar algunos metadatos del servicio. El proceso de publicación es responsable de que el servicio generado esté disponible, a través del framework, para que los potenciales usuarios finales puedan consumirlos.

Un repositorio distribuido es utilizado para almacenar referencias a los servicios, las cuales están indexadas por categorías. Las categorías se resuelven durante el proceso de clasificación del servicio. Una vez que se coloca el código de servicio en el repositorio externo, el siguiente proceso es la búsqueda, este permite que los usuario inicien la búsqueda de servicios, ya sea de forma automática, o mediante mecanismos de multicast como los definidos en WS-Discovery specification ("Web Services Dynamic Discovery (WS-Discovery) Version 1.1," 2009), o manualmente introduciendo algunos parámetros como palabras claves en un motor de búsqueda. Cuando un servicio es encontrado y recuperado, puede ser adaptado según las preferencias del usuario o información del entorno. El procedimiento de personalización en el proceso de adaptación del servicio, es diferente de la técnica de configuración en términos de flexibilidad y complejidad, debido a la personalización a menudo implica cambios en 
el código fuente para crear funcionalidades que están un paso más allá del límite de lo configurable. La configuración por lo general se apoya en cambios a través de la configuración de parámetros predefinidos de alcance restringido (Sun, Zhang, Guo, Sun, y Su, 2008).

El proceso de ejecución inicia cuando un usuario ha descubierto y opcionalmente adaptado un servicio. El último proceso del ciclo de vida es la verificación, mediante el cual los usuarios pueden confirmar si los servicios se han ejecutado completamente.

El modelo de ciclo de vida de servicios tolerantes mostrado en la Figura 9, contempla varias trayectorias, donde los procesos por los que atraviesa el servicio durante su ciclo de vida, varían en función de las necesidades del usuario. Las trayectorias posibles durante el ciclo de vida de los servicios tolerantes de describen a continuación:

Creación desde cero: está definida por el itinerario que pasa por los procesos de creación, clasificación y publicación. Considera que el usuario utiliza únicamente las herramientas disponibles en el framework de servicios para la creación del servicio. No contempla la reutilización de servicios predefinidos. Este caso culmina con la publicación del servicio.

Creación desde plantilla: definida por el itinerario que atraviesa por los procesos de búsqueda, adaptación, clasificación y publicación. Esta alternativa permite a un usuario crear un servicio, basado en el trabajo de otro usuario. El usuario inicia buscando las plantillas de servicios disponibles, y adapta la plantilla seleccionada a sus necesidades.

Personalización de plantilla: definida por el itinerario que pasa por los procesos de búsqueda, adaptación y ejecución. Esta alternativa considera a un usuario que requiere utilizar un servicio personalizable en forma de una plantilla, de modo que el usuario inicia el caso de uso en busca de una plantilla que pueda adaptar y posteriormente ejecutar.

Ejecución final del servicio: está definida por el itinerario que recorre los procesos de búsqueda, ejecución y opcionalmente verificación. Considera a un usuario con la intención de consumir un servicio final, sin pasar por el proceso de adaptación. Supone que el servicio requerido es encontrado en el proceso de búsqueda, y que se adapta al objetivo perseguido por el usuario, o es necesaria una pequeña personalización que no se considera como adaptación.

\subsubsection{Proceso de creación}

En este proceso se lleva a cabo la fabricación del servicio, y se descompone básicamente en tres actividades a saber: identificación del servicio, identificación de los elementos del sistema y construcción del servicio.

En el proceso de creación de servicio los insumos de entrada son la experiencia del dominio y la experiencia de creación de servicios, además de unas guías que consisten en material de capacitación. El material de entrenamiento es generado por el grupo de 
desarrolladores del framework, que son quienes previamente entrenan a los usuarios en el uso del framework (guías, manuales y tutoriales).

Las tres actividades del proceso de creación son divididas en tareas que son asignadas al usuario y al especialista de framework. El procedimiento de intercambio de productos de trabajo se muestra en la Figura 10.

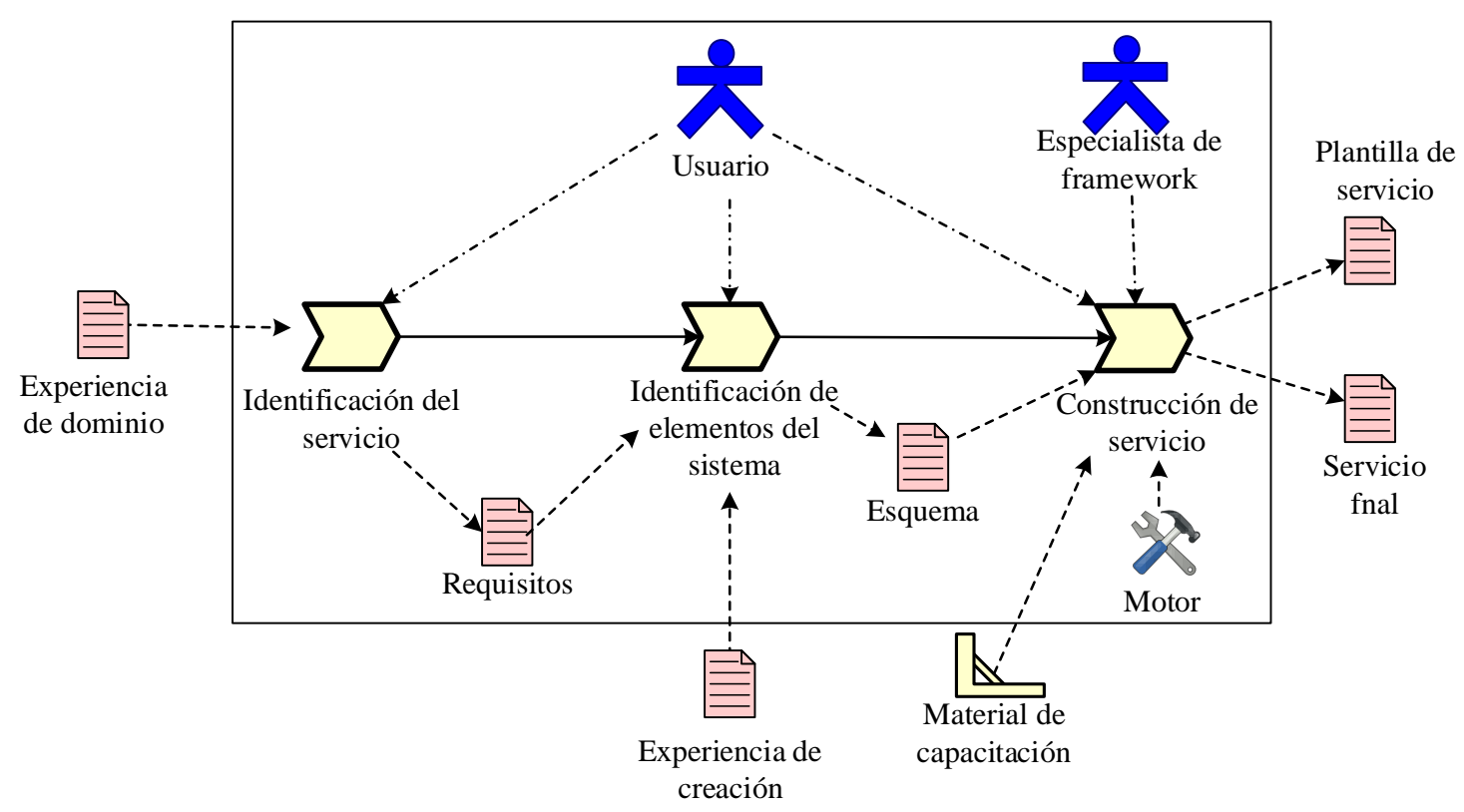

Figura 10. Proceso de creación.

\section{Identificación del servicio}

Esta tarea inicia cuando el usuario ha decidido crear el servicio que cumpla con sus objetivos. Para esto cuenta con su experiencia en la creación y el conocimiento del escenario de aplicación. En esta tarea, el usuario crea el servicio necesario para resolver un problema. El producto de esta tarea es el establecimiento de los requisitos, que es uno de los insumos necesarios en la próxima tarea.

\section{Identificación de elementos del sistema}

En esta tarea el usuario identifica los elementos que ofrece el framework para satisfacer sus necesidades de servicio. Las entradas de esta tarea son la experiencia del usuario utilizando el framework y un conjunto de requisitos de creación, que se transforman a un esquema de creación, es decir, la idea de cómo componer el servicio teniendo en cuenta los elementos soportados por el framework.

\section{Construcción del servicio}

Esta tarea inicia una vez que finaliza la tarea de identificación de elementos del sistema. En esta tarea el usuario sabe cómo construir el servicio por su cuenta. Como insumo para realizar esta tarea, el usuario tiene el esquema de creación, y también el 
material de capacitación modelado en SPEM, que se ha mejorado en las diferentes iteraciones de creación de servicios.

Un tema común en este paso es que los usuario se bloquean en alguna parte de la fase de creación, debido a la falta de conocimiento completo del entorno de creación, la falta de experiencia u orientación de por parte de los expertos. Por lo tanto, un especialista de framework asiste al usuario cuando se queda atascado.

El resultado de esta tarea, generada con la ayuda de una herramienta llamada sistema de creación, es una plantilla de servicio o un servicio final, dependiendo de la estrategia de creación de implementada en el framework.

\subsubsection{Proceso de clasificación}

El proceso de clasificación consiste en la categorización de los servicios de acuerdo sus tópicos, y su indexación en el repositorio de servicios. Una correcta clasificación permite una efectiva búsqueda de servicios. La Figura 11 describe la tarea de clasificación, los roles y los productos de trabajo relacionados.

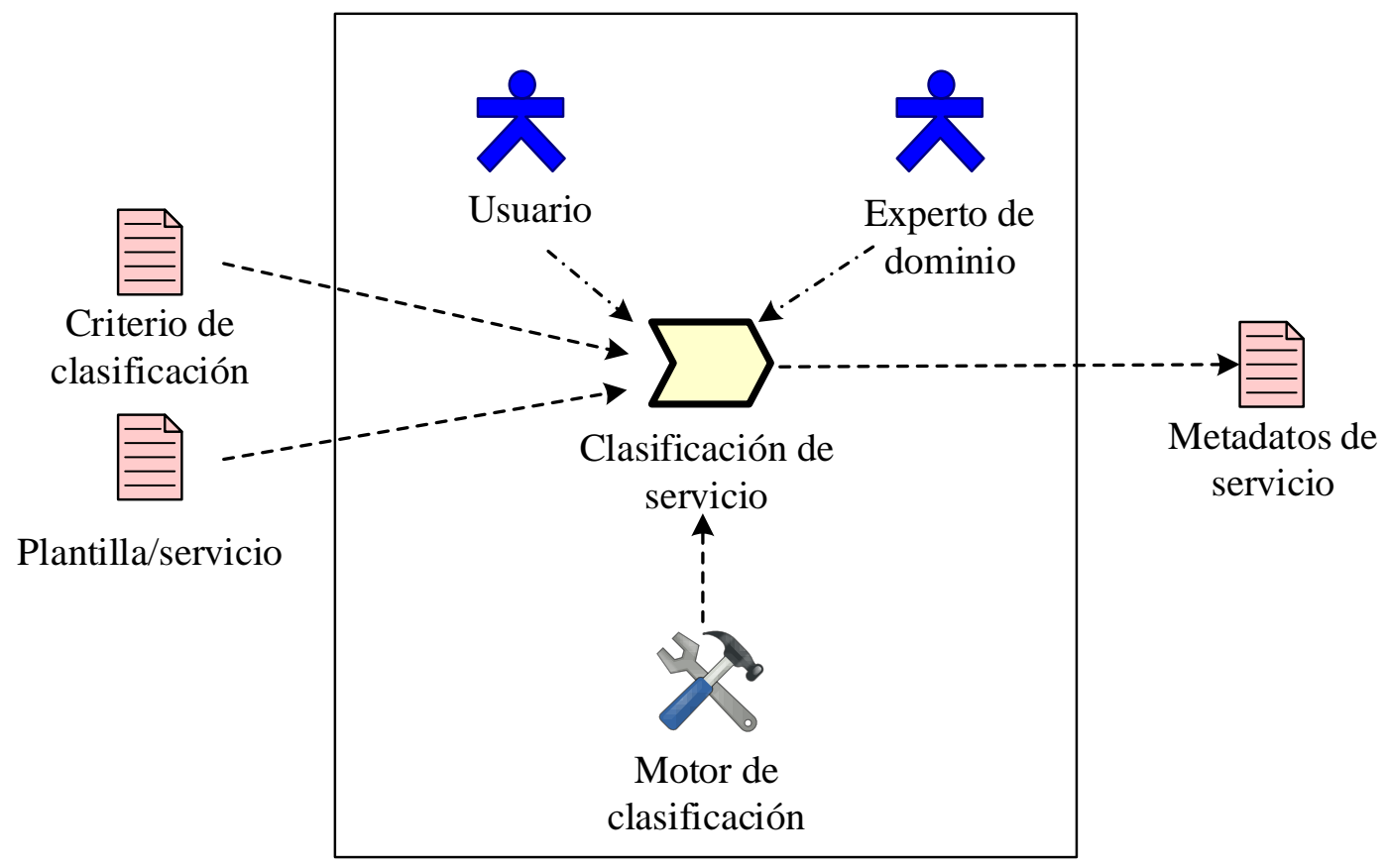

Figura 11. Proceso de clasificación.

El proceso de clasificación del servicio requiere como entrada la plantilla o servicio a clasificar, y un conjunto de criterios relacionados con el entorno de aplicación. El experto de dominio asiste al usuario para clasificar convenientemente el servicio generado, de acuerdo a su conocimiento del entorno. La salida de la fase de clasificación es un conjunto de metadatos de servicio, que se generan de forma automática o semi-automática a través de una herramienta denominada motor de 
clasificación. Los metadatos generados son el insumo de entrada para el siguiente proceso del ciclo de vida del servicio.

\subsubsection{Proceso de publicación}

El objetivo principal de este proceso de publicación es que el servicio generado esté disponible a través del framework para que los potenciales usuarios puedan consumirlo. Un repositorio distribuido se utiliza para almacenar las referencias a los servicios, indexadas por categorías. La Figura 12 describe la tarea de publicación, los roles y los productos de trabajo relacionados.

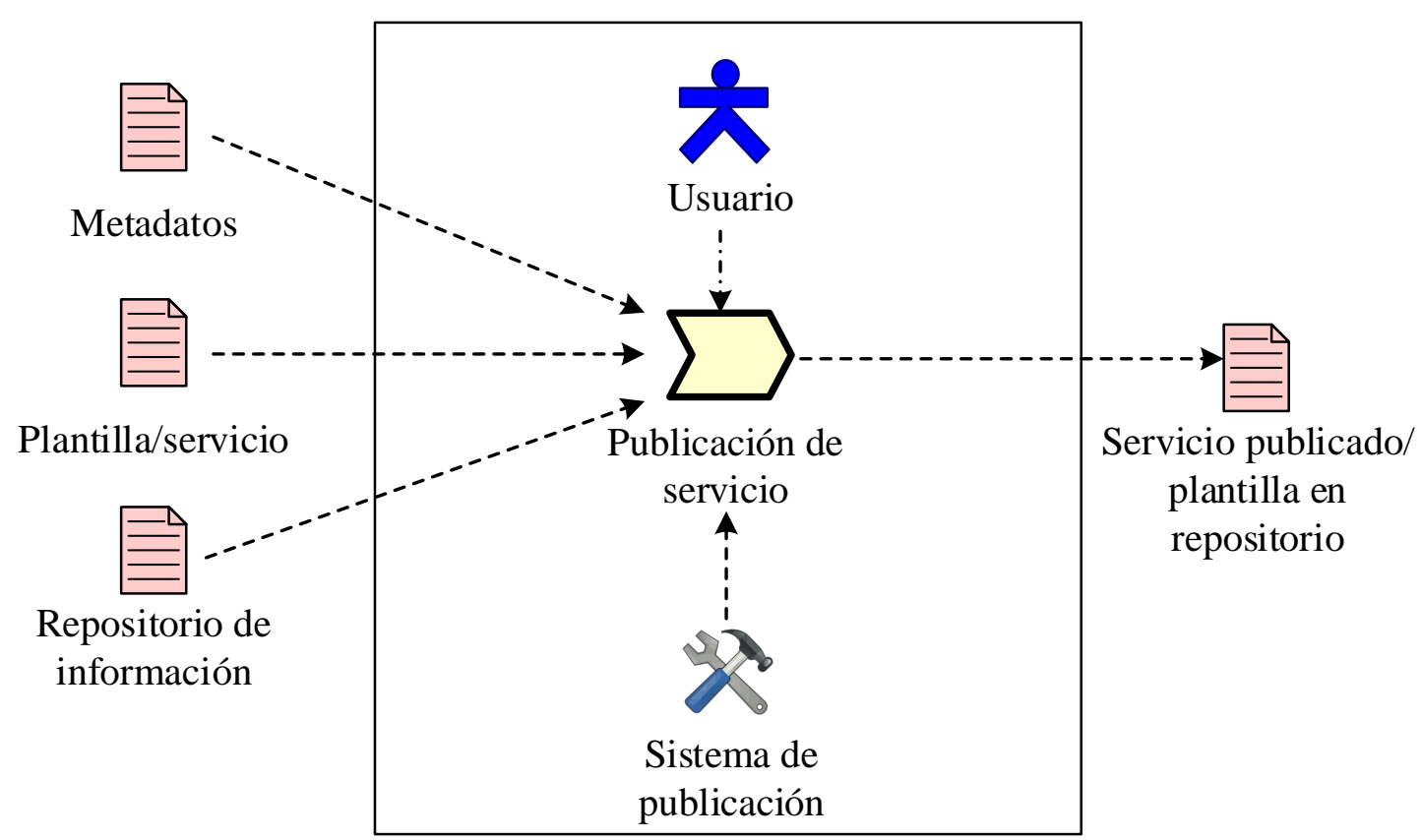

Figura 12. Proceso de publicación.

El proceso de publicación de servicio necesita como entrada el servicio o plantilla objeto de la publicación, el producto de los metadatos generados en proceso de clasificación y la información del repositorio, para realizar el procedimiento de publicación. La salida del proceso de publicación es el servicio o plantilla debidamente publicados en el repositorio, mediante una herramienta llamada sistema de publicación.

\subsubsection{Proceso de búsqueda}

Este proceso permite a los usuarios recuperar un servicio o plantilla de un repositorio externo. La Figura 13, describe la tarea de búsqueda, los roles y sus productos de trabajo. 


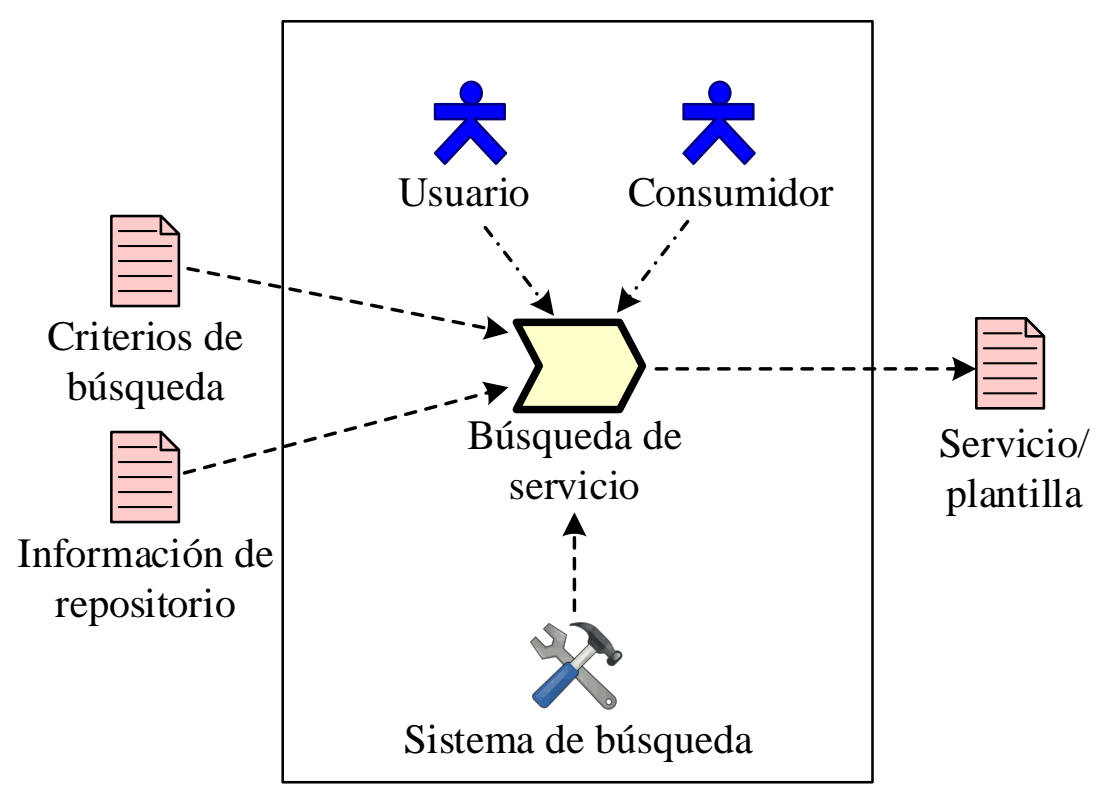

Figura 13. Proceso de búsqueda.

Este proceso está compuesto por una sola tarea, la de buscar. Para esta tarea es necesario como insumos, los siguientes productos: los criterios de búsqueda y la información del repositorio. Esta tarea puede ser realizada por el consumidor o por el usuario actuando en el rol del consumidor. La salida de este proceso es el servicio o plantilla, entregada por el sistema de búsqueda.

\subsubsection{Proceso de adaptación}

El proceso de adaptación está conformado por dos tareas, previsión de la adaptación y personalización del servicio, las cuales son asignadas a los roles de usuario y consumidor respectivamente. El intercambio de productos de trabajo se representa en la Figura 14.

Los productos de entrada son la experiencia en el dominio, la experiencia en la creación de servicios y la plantilla. Adicionalmente, se incorporan al proceso de adaptación una guía, que consiste en material de capacitación generado en el entrenamiento. 


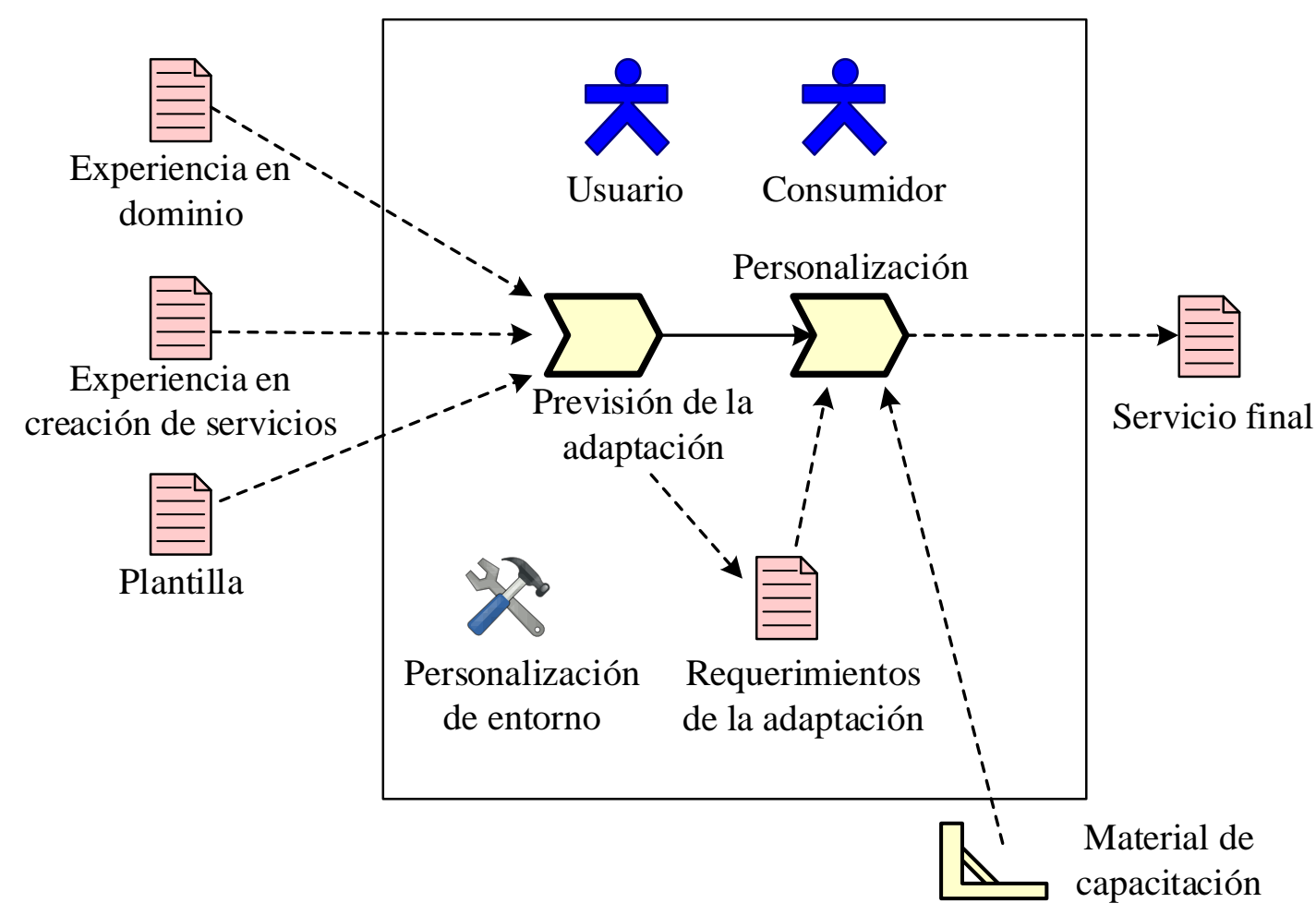

Figura 14. Proceso de adaptación.

\section{Previsión de la adaptación}

Esta tarea inicia una vez ha recibido la plantilla del servicio por el consumidor o el usuario. El objetivo de esta tarea es determinar qué nivel de adaptación requiere la plantilla del servicio, y cómo la personalización de servicios debe ser para satisfacer los objetivos del usuario. Para esto es necesario contar con los productos de trabajo: experiencia de dominio, la experiencia en creación de servicios y la plantilla de servicio.

El producto de trabajo resultante de esta tarea son un conjunto de requerimientos de adaptación que serán usados como insumo de entrada en la tarea de personalización.

\section{Personalización del servicio}

Esta tarea es ejecutada una vez que la tarea de previsión de la adaptación se ha completado. En esta tarea el usuario realiza la personalización sobre la plantilla del servicio. Como insumos de esta tarea son necesarios los productos de trabajo, requerimientos de adaptación y también el material de capacitación, modelado como una guía en SPEM. El material de capacitación ha sido mejorado en función de varias iteraciones en la creación de servicios. La salida de esta tarea y del el proceso de adaptación en sí, es el servicio final. 


\subsubsection{Proceso de ejecución}

El proceso de ejecución se descompone en tres tareas, ejecución del servicio, resolución del servicio y acceso a recursos, las cuales están asignadas al rol del usuario o usuarios que ejecutan el servicio, en este caso puede ser o bien un usuario o un consumidor normal. Como se muestra en la Figura 15, estas tareas reciben los productos de trabajo: participantes en la ejecución y el servicio, y generan como consecuencia los productos de trabajo, resultados de ejecución e informe de ejecución. El producto de trabajo informe de ejecución, es generado por el usuario de forma manual o semi-automática, y contiene información de carácter no técnico, sobre el rendimiento del servicio y su utilidad. El producto resultados de ejecución es un informe generado automáticamente que contiene la información del registro de ejecución o el monitoreo de las actividades ocurridas durante la ejecución del servicio.

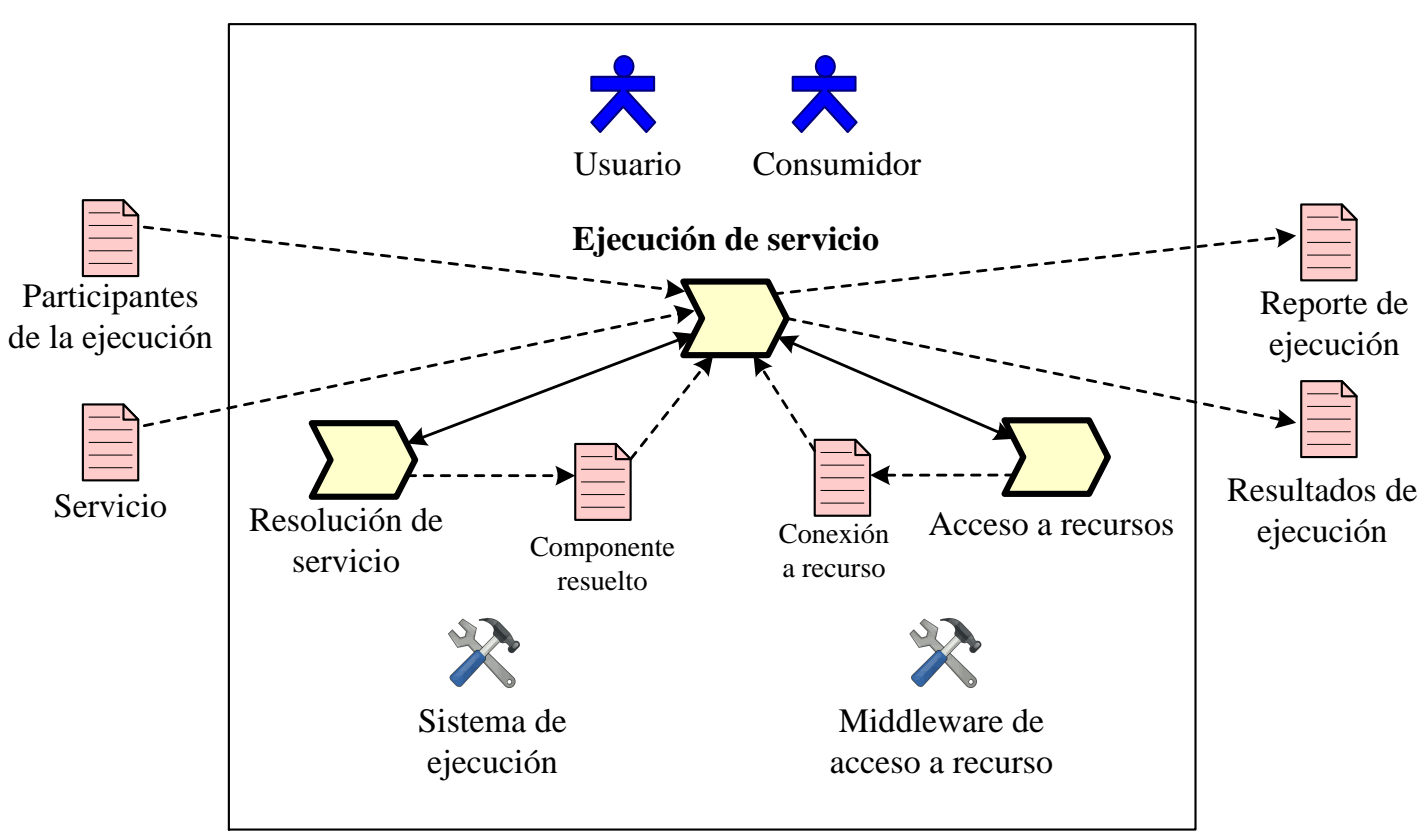

Figura 15. Proceso de ejecución.

\section{Ejecución de servicio}

El objetivo de esta tarea es ejecutar el servicio solicitado por el usuario. Esta tarea utiliza la herramienta sistema de ejecución, los participantes de la ejecución y el servicio de entrada. Los participantes de la ejecución es un producto de trabajo necesario para determinar el número de entidades que pueden participar en la ejecución. Las salidas de esta tarea son los productos de trabajo reporte de ejecución y resultados de la ejecución. 


\section{Resolución de servicio}

El objetivo de esta tarea es ejecutar el proceso de resolución. Este proceso asigna, en tiempo de ejecución, a cada componente la mejor capacidad disponible que puede implementar. En este proceso tiene lugar el MatchMaking, que es el procedimiento de buscar la capacidad óptima que satisface los requerimientos del componente. Encontrar la capacidad óptima depende de varios factores, por ejemplo, las opciones de configuración establecidas por el usuario durante la creación, o requisitos de los componentes. Con el fin de ofrecer una experiencia de ejecución continúa el MatchMaking busca y cambia las capacidades utilizadas para resolver un componente de forma dinámica, transparente y autónoma. Por lo tanto, un componente se traduce a una capacidad antes de su invocación.

La salida de esta tarea es componente resuelto, el cual sirve de entrada para la tarea de ejecución de servicio.

\section{Acceso a recurso}

El objetivo de la tarea acceso a recursos es facilitar el acceso homogéneo a los recursos proporcionados por capacidades heterogéneas. La herramienta utilizada en esta tarea se llama middleware de acceso a recursos, que consiste en un algoritmo de descubrimiento, que es ejecutado de forma permanente por las entidades móviles que están presentes en el escenario planteado. La salida de esta tarea es un producto de trabajo denominado conexión a recurso, que puede ser demandado por la tarea de ejecución de servicio para permitir el consumo de recursos.

\subsubsection{Proceso de verificación}

En el proceso de verificación se evalúa si el servicio creado cumple las expectativas del usuario o consumidor, y trata de corregir las desviaciones entre las intenciones al crear el servicio y la funcionalidad que el servicio finalmente ofrece. Para asegurar su integridad, esta fase debe verificar que todas las funcionalidades de la ejecución del servicio ejecutado se pusieron a prueba, y la guía de material de capacitación fue convenientemente seguidas. 
La Figura 16, describe las tareas de verificación y cotejo; los roles y los productos de trabajo.

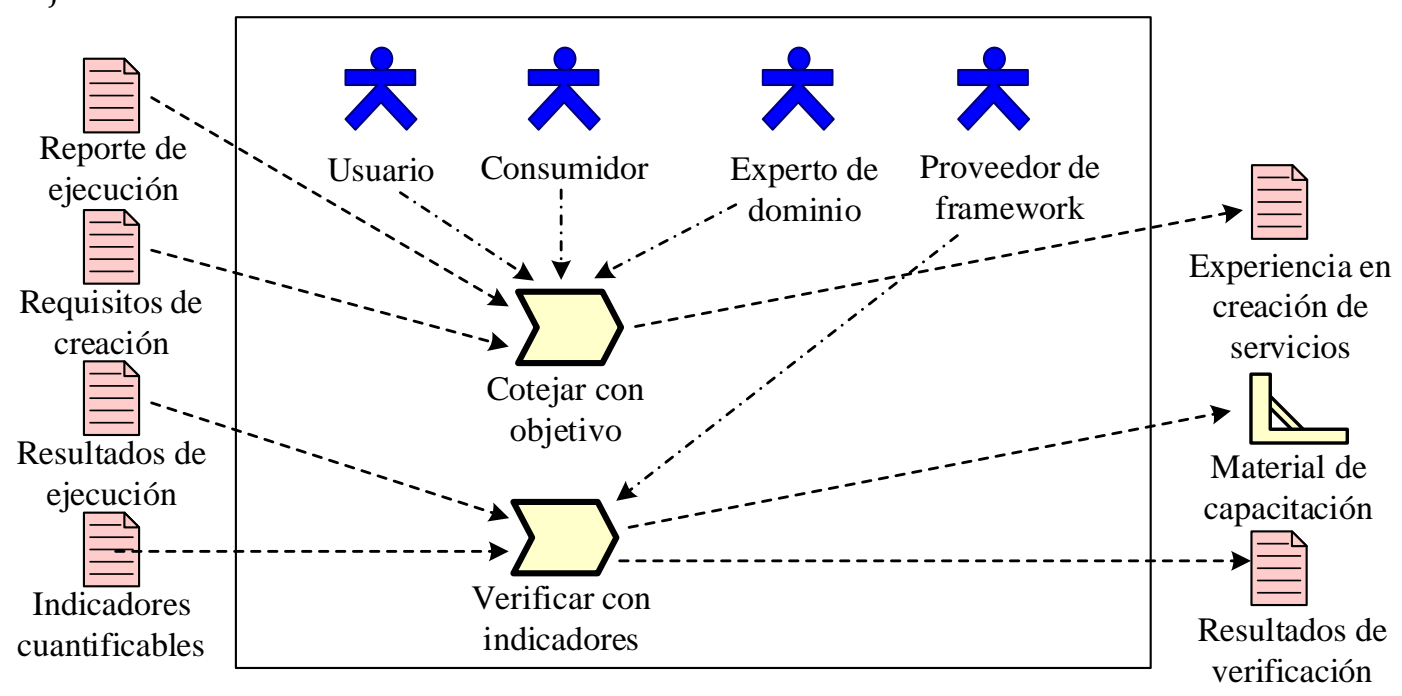

Figura 16. Proceso de verificación.

Este proceso contempla dos tareas. La primera tarea, cotejar contra objetivo, verifica que la ejecución del servicio es conforme o intenta cubrir los requerimientos del servicio. Esta tarea requiere como entrada los productos de trabajo, reporte de ejecución y requisitos de creación, con el fin de realizar la comparación. Ya sea un usuario, un consumidor o un experto de dominio pueden realizar esta tarea, ya que el informe de ejecución es un documento generado en el proceso de ejecución que contiene información no técnica sobre el rendimiento de los servicios y su utilidad. La salida de esta tarea es el producto de trabajo experiencia de creación de servicios, que representa un incremento en el conocimiento para el consumidor/usuario/experto de dominio.

La segunda tarea, verificar con indicadores, compara los resultados de la ejecución del servicio, a través de registros de ejecución o el monitoreo de las actividades de ejecución, con indicadores medibles, tales como el exceso de confianza del usuario en la tarea de creación, la falta de un amplio conocimiento del entorno o los problemas en la comprensión del material de capacitación. Esta tarea es realizada por el proveedor de framework, ya que para entender el paquete de trabajo resultado de la ejecución, se requiere un rol con experiencia en tecnología. La salida de esta tarea es un documento llamado resultados de verificación, que constituye una retroalimentación para el proveedor de framework, que se utiliza para mejorar el diseño de la aplicación y del framework de servicios, y una versión mejorada del material de capacitación, para disposición del usuario del framework. 


\subsection{Conclusiones}

En esta sección se define la capa de gestión de servicios y comunicaciones, para redes de sensores en entornos remotos, basados en tecnologías DTN. Se introducen los conceptos de servicio y servicio tolerante y se profundiza en los procesos de delegación, desacople y atomicidad, fundamentales para entender las contribuciones posteriores.

Utilizando el lenguaje UML y SPEM se definen los elementos fundamentales de un servicio y su ciclo de vida, considerando que atraviesa los procesos de creación, clasificación, publicación, adaptación, búsqueda, ejecución, y verificación.

Las contribuciones del trabajo de tesis se centran principalmente en el proceso de ejecución, en el que se producen dos subprocesos fundamentales para lograr una ejecución continua de servicios en entornos disruptivos, la resolución de servicios y el acceso continuo a recursos.

En el proceso de resolución de servicios, el matchmaker de capacidades busca y cambia las capacidades utilizadas para resolver un componente de forma dinámica, transparente y autónoma. Por lo tanto, un componente se traduce a una capacidad antes de su invocación. En el proceso de acceso a recursos, el middleware de acceso facilita el acceso homogéneo a los recursos proporcionados por capacidades heterogéneas. 


\section{Provisión dinámica para la ejecución de servicios}

\subsection{Introducción}

En este capítulo, propongo un framework de ejecución de servicios distribuidos tolerantes al retardo a alto nivel, que permita la identificar aquellos servicios elegibles para ser ejecutados sobre una determinada infraestructura (Objetivo \#6), en un entorno como se ha definido en la arquitectura general en la Sección 4.3.

La estrategia de ejecución, permite un reparto del servicio entre los dispositivos móviles, asegurando gran flexibilidad en ambientes con conectividad altamente itinerante. Para esto se hace necesario resolver desafíos de coordinación con el objetivo de lograr una ejecución eficiente en entornos específicos de redes de sensores y actuadores con conectividad limitada. En términos de la comunicación, propongo la utilización del paradigma de publicación y suscripción.

La fragmentación de los servicios la analizo en la Sección 6.2, en donde propongo un esquema de identificación de las instancias, posteriormente desarrollo varios esquemas de transformación de servicios en función del tipo de actividades que contiene. En la sección 6.3 se describe como pueden descubrirse los elementos básicos que componen un servicio y que dependen de la WSAN, y en la seción 6.4 se propone un esquema para hallar als correspondencias entre los fragemntos de un servicio y los elementos descrubiertos en la WSAN.

\subsection{Modelo de Ejecución de Servicios}

La organización de la arquitectura propuesta en el capítulo 4, presenta varios desafíos especiales en relación a la ejecución de servicios móviles en entornos de WSAN con comunicaciones limitadas. De acuerdo a las características de la capa DTN de la arquitectura física descrita en la sección 4.5.2, existe un conjunto de participantes 
móviles en la ejecución de los servicios, por lo tanto, desde el plano de los servicios el modelo debe proporcionar soporte para gestión de eventos frecuentes en entornos de movilidad, como son las interrupciones en la ejecución de los servicios, retardos, suspensión y reanudación. Adicionalmente, debe proveer mecanismos de persistencia del servicio, así como también de su estado.

Por otro lado, desde la perspectiva del hardware de los nodos asociados al servicio, hay que tomar en cuenta las características limitadas con que cuentan los participantes móviles y los dispositivos finales en las WSAN. Otros requerimientos importantes que deben ser soportados son los que se derivan de las limitadas y discontinuas comunicaciones que se pueden establecer con los dispositivos de tipo cluster head.

El modelo propuesto considera los desafíos de la arquitectura propuesta, ofreciendo soporte a las múltiples adaptaciones del servicio en tiempo de ejecución, producto de la heterogeneidad de los recursos tecnológicos en los entornos de WSAN y dispositivos móviles. Las principales estrategias de ejecución de servicios consisten en la resolución dinámica de los componentes, y la asociación temporal a los recursos conocidos mediante el proceso de descubrimiento. Este modelo permite obtener un desacople de todos los elementos que integran el sistema, es decir, entre los servicios, las comunicaciones y los dispositivos finales en las WSAN.

\subsubsection{Conceptos fundamentales}

En el modelo propuesto para la ejecución de servicios móviles en ambientes de WSAN aisladas, remotas o con comunicaciones limitadas, se definen algunos conceptos que son fundamentales para estructurar la visión general del mismo: servicio, componente, capacidad, dispositivo y recurso.

Servicio: el concepto de servicio ya ha sido definido en la sección 5.3 como servicio tolerante. Un servicio tolerante está compuesto por uno o más componentes, que se relacionan lógicamente, y se comunican entre sí para llevar a cabo una misión específica. El proceso de creación del servicio es realizado por los usuarios utilizando el framework de desarrollo de servicios, obteniendo como producto una plantilla o preservicio publicado. La plantilla del servicio incluye metadata acerca del servicio (creador, fecha, etc.), lógica y la descripción de la funcionalidad, además de una lista de componentes requeridos para su ejecución.

Componente: es una abstracción de una funcionalidad, es decir, es un fragmento del servicio que debe realizar una tarea concreta. Esta tarea está relacionada con una capacidad que le permite resolver la tarea. La capacidad debe proveer acceso a los recursos requeridos por el componente. Los componentes son piezas que se utilizan en el proceso de creación, y ayudan a los usuarios a seleccionar las funcionalidades del servicio con un mayor nivel de granularidad. Los componentes permiten el desacople de la funcionalidad que el usuario requiere en el servicio, de la implementación real de dicha funcionalidad. 
Capacidad: es un concepto mediante el cual se implementan las funcionalidades de los componentes. Las capacidades proveen acceso a las facilidades que ellos representan y al mismo tiempo ocultan los detalles de los recursos subyacentes. Una capacidad puede ser implementada utilizando recursos de software o hardware, mientras provee una vista abstracta de los recursos latentes. Las capacidades incluyen metadata que define sus propiedades, esta información es requerida durante el proceso de resolución de los componentes con el objetivo de seleccionar la capacidad más adecuada para cada componente. Las capacidades tienen dos interfaces, una con los componentes y otra con los dispositivos físicos, quienes ofrecen finalmente los recursos disponibles en las WSAN.

Dispositivo: son elementos físicos que gestionan un conjunto de recursos (sensores y actuadores), generalmente denominados cluster head en WSAN. Un dispositivo está provisto de mayores recursos de hardware que los sensores y actuadores que gestiona, además, dispone de mayores capacidades en software que le permiten ofrecer sus recursos a otros dispositivos o equipos circundantes que lo requieran. Un dispositivo ofrece sus servicios mediante publicaciones DTN periódicas.

Recurso: son las unidades más elementales de la arquitectura propuesta, sin embargo, no por ello menos importantes. Los recursos son los que realmente permiten materializar los servicios tolerantes, realizando las acciones requeridas por el servicio. Consisten en el conjunto de sensores y actuadores que conforman las WSAN. Los recursos son entidades atómicas e individuales, sin embargo, están sujetas a coordinación por parte de su cluster head, y se conceptualizan como participantes de los servicios, en la visión general de la arquitectura.

En la Figura 17, se puede observar un esquema general del modelo desde dos perspectivas, la vista lógica y la vista física. La visión lógica nos permite identificar las posibles interrelaciones entre los conceptos anteriormente definidos, y las interfaces de asociación que deben existir con sus vecinos. En esta perspectiva se denota el desacoplamiento entre componentes, capacidades y dispositivos. Por su parte el plano físico muestra el desacoplamiento temporal y espacial entre los componentes de la arquitectura.

Estos dos planos se vinculan mediante la relación existente entre los conceptos y dónde se implementan los mismos. En la Figura 17, podemos percibir que los servicios se crean mediante un framework para desarrollo de servicios alojado en la cloud. Por su parte, el repositorio de capacidades está a bordo de entidades móviles denominadas mulas, y que además, tienen como objetivo transportar los mensajes de comunicación que deben intercambiarse entre los servicios y los dispositivos. Los dispositivos se implementan físicamente en los cluster head, es decir, los nodos coordinadores de las WSAN que se encuentran ubicadas en sitios remotos. Un dispositivo puede estar asociado a uno o más recursos. Adicionalmente, los recursos están constituidos por los sensores, actuadores y otras facilidades proporcionadas por las WSAN. La ubicación 
física de los recursos está definida por la ubicación geográfica del despliegue de la WSAN a la que pertenece.

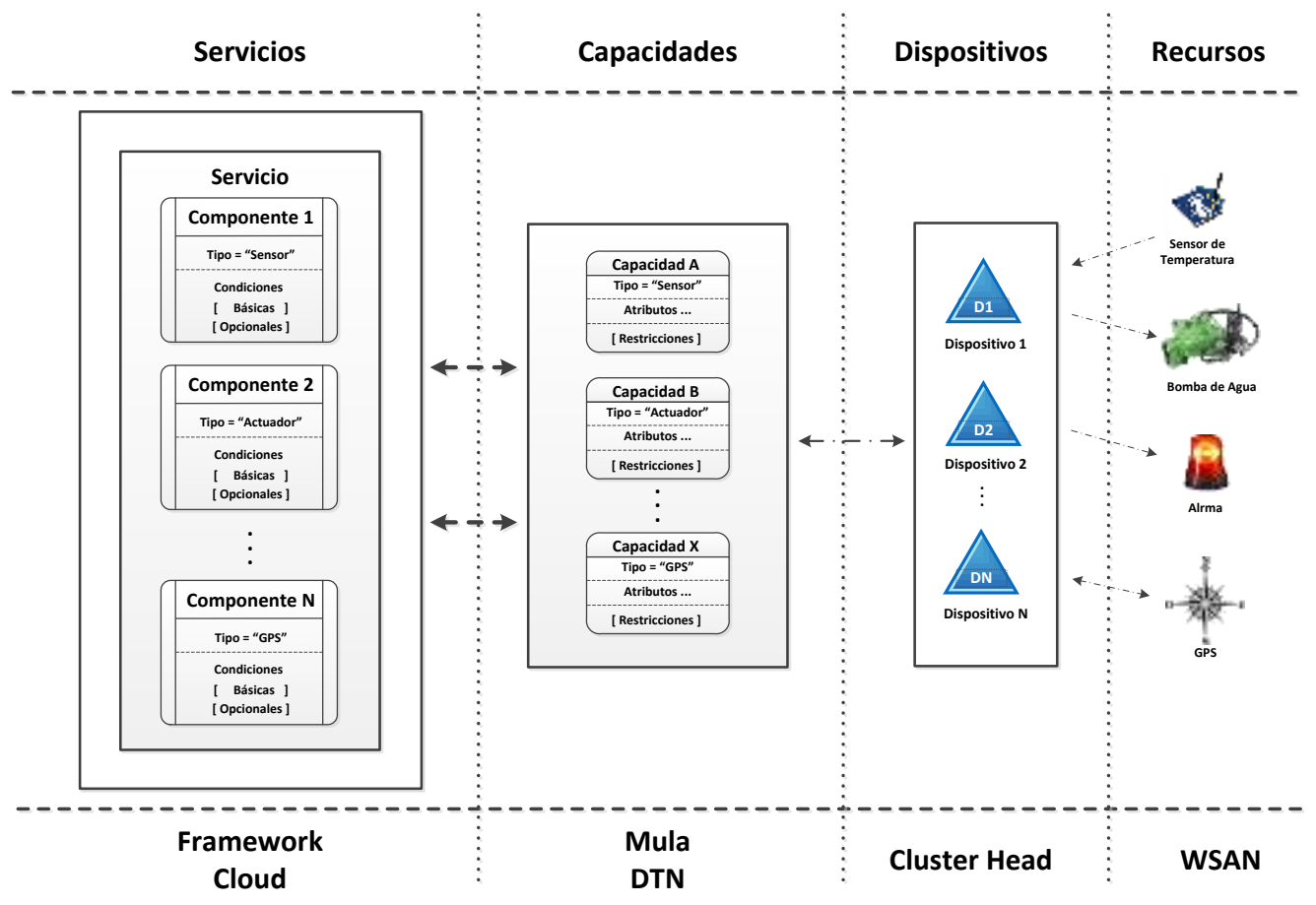

Figura 17. Visión global del modelo.

\subsubsection{Resolución dinámica de componentes}

La unidad elemental de composición de los servicios es el componente, sin embargo, para ser útiles deben encontrar en tiempo de ejecución una capacidad adecuada que les permita realizar su función de la forma más óptima posible. Para llevar a cabo esta asociación es necesario considerar las condiciones básicas y opcionales impuestas por el creador del servicio. De igual forma las restricciones impuestas por las capacidades condicionan el proceso de resolución.

El proceso de resolución dinámica de componentes se realiza en tiempo de ejecución del servicio, por lo tanto, las oportunidades de asociación con las capacidades tienen una temporalidad acotada, esto debido a la movilidad de las entidades portadoras de las capacidades (mulas DTN). El tiempo disponible para realizar en proceso de asociación está determinado por la visibilidad de la entidad móvil en la red con acceso a la cloud. Esta circunstancia se presenta de igual forma para la entrega de los resultados de la ejecución de la capacidad.

Un componente puede asociarse a capacidades más o menos compatibles, donde la compatibilidad está definida por el nivel funcional de la capacidad sujeto de la 
vinculación. Los componentes definen condiciones básicas y opcionales para su ejecución. Las condiciones básicas deben ser resueltas totalmente por la capacidad, para asegurar la correcta ejecución del componente, mientras las opcionales pueden enriquecer la funcionalidad del componente.

La asociación asíncrona entre los componentes y las capacidades se realiza de forma continua, es decir, un componente que posee un nexo con una capacidad concreta, puede encontrar otra capacidad que es más conveniente, en un repositorio de una entidad móvil distinta, y asociarse a ella. Esta característica permite el desacoplamiento espacial y temporal entre los componentes y las capacidades que implementan sus funcionalidades. El procedimiento de resolución o asociación de los componentes es llevado a cabo por el algoritmo de MatchMaking (MM), que se desarrolla posteriormente en la sección 6.4.

En el esquema de la Figura 18, se puede observar el desacoplamiento entre los componentes de servicios y las capacidades que implementan las funcionalidades. El software que realiza el proceso de resolución o asociación se ejecuta del lado del repositorio de servicios (servidor en cloud), ya que este anfitrión del repositorio de servicios, generalmente tendrá mayores recursos (cpu, ram, almacenamiento, etc.) de cómputo para resolver esta compleja tarea.

El esquema funcional, revela que las capacidades están constituidas por dos interfaces, una es la interface de asociación con los componentes, y la otra es la interfaz de reconocimiento de recursos, a través de los dispositivos. Este reconocimiento de recursos es realizado por el algoritmo de descubrimiento que se explica en la sección 6.2.3. La interfaz de comunicación con los servicios provee una abstracta vista pública, mientras oculta las características específicas de los recursos.

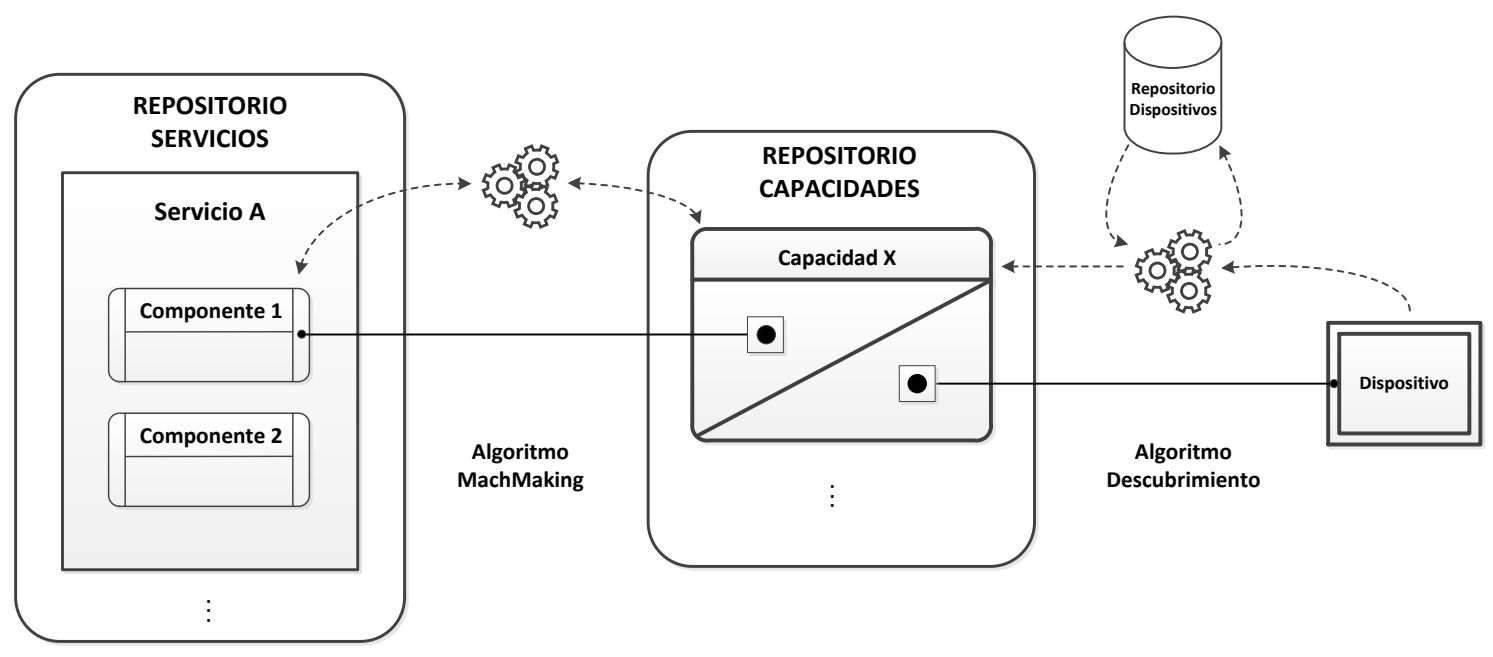

Figura 18: Esquema funcional del modelo

Las capacidades pueden ser clasificadas de dos formas principalmente, las primeras son las capacidades internas, estas pueden ser resueltas por los recursos locales del 
portador de la capacidad (en móvil: cámara, gps, mapas, acelerómetro, etc.). Las segundas son las externas, es decir, las que están relacionadas con recursos ubicados en el contexto circundante, o remotos, en cuyo caso se requiere de comunicaciones para poder acceder a ellos (sensores y actuadores en WSAN).

Esta arquitectura funcional permite una dinámica y continua resolución de componentes, creando una correlación entre las capacidades que proporcionen las mejores características según los requisitos definidos por el creador del servicio y las restricciones definidas por las capacidades.

\subsubsection{Reconocimiento oportunista de recursos}

En la Figura 18, se puede observar la relación de las capacidades con los dispositivos (cluster head), estos últimos son los que exponen los a las entidades del contexto circundante, los recursos disponibles en sus WSAN. Los dispositivos ofrecen sus recursos por medio de una publicación periódica (beacon DTN), de esta forma los vecinos dentro de la cobertura reconocen oportunamente los recursos disponibles. Las entidades móviles que tienen eventualmente contacto con los dispositivos, agregan los dispositivos contactados a un repositorio, que se aloja de forma local conjuntamente con el repositorio de capacidades. Este repositorio de dispositivos es procesado por el algoritmo de descubrimiento, explicado en la sección 6.3. El algoritmo permite crear las capacidades, a partir de la información de los recursos disponibles ofrecidos por cada dispositivo cluster head contactado.

Pueden existir uno o más dispositivos conocidos mediante descubrimiento, que ofrezcan los recursos necesarios para ejecutar una o varias capacidades de los componentes de servicio. La entidad móvil que es anfitriona de un repositorio de capacidades, también puede ser capaz de solicitar la ejecución una acción sobre un recurso, siempre que pueda comunicarse con el dispositivo que los coordina (cluster head), siguiendo una filosofía oportunista.

\subsection{Descubrimiento de dispositivos}

Como se ha comentado en la sección reconocimientos oportunistas de recursos, el proceso de descubrimiento permite conocer los dispositivos dentro del área de cobertura de la interfaz de comunicación de la entidad móvil que ejecuta el algoritmo de descubrimiento. Este algoritmo está continuamente activo en un equipo móvil con capacidades DTN, que puede tener contactos con los dispositivos que proveen los recursos, y por otro lado, con la infraestructura de comunicación que le permite obtener acceso a la cloud. En términos de la tecnología DTN a esta entidad se le denomina mula.

El algoritmo considera un entorno de comunicaciones limitado, en donde eventualmente la mula tendrá oportunidad de intercambiar mensajes con los cluster head, durante periodos cortos de tiempo. El tiempo de contacto entre la mula y un dispositivo está dado en función principalmente de dos factores, la velocidad con que 
pasa la mula y el poder de procesamiento de ambos equipos para tratamiento los mensajes de comunicación. El funcionamiento del algoritmo de describe en la siguiente sección.

\subsubsection{Algoritmo de descubrimiento}

El proceso de descubrimiento se ilustra en el Algoritmo 1, este software se ejecuta permanentemente en las mulas, y tiene como objetivo escuchar los beacom DTN de los dispositivos ( $\boldsymbol{D})$ que anuncian su presencia (2). Cuando la mula detecta la presencia de un dispositivo a través de su anuncio, solicita los datos de los recursos subyacentes a ese dispositivo concreto. Si el dispositivo detectado no está registrado en el repositorio de dispositivos $(\boldsymbol{R} \boldsymbol{d})$, este es agregado (5). Las capacidades se clasifican por tipos (sensor, actuador, gps, etc.), y se crea una capacidad para cada tipo, las nuevas capacidades son registradas (8) en el repositorio de capacidades $(\boldsymbol{R} \boldsymbol{c})$. Estas se agregan en función de la aparición de nuevos recursos disponibles. Una vez que un nuevo dispositivo es agregado, se registran cada uno de sus recursos disponibles $\left(\boldsymbol{R}_{k}\right)$, asociados a al tipo de capacidad correspondiente, así como toda la información inherente al recurso (tipo de capacidad, tipo de recurso, nombre de dispositivo, estado, restricciones, atributos, etc.). En el caso de registro previo del dispositivo, se verifica la disponibilidad de los recursos, para actualizar su estado en el repositorio de capacidades (12).

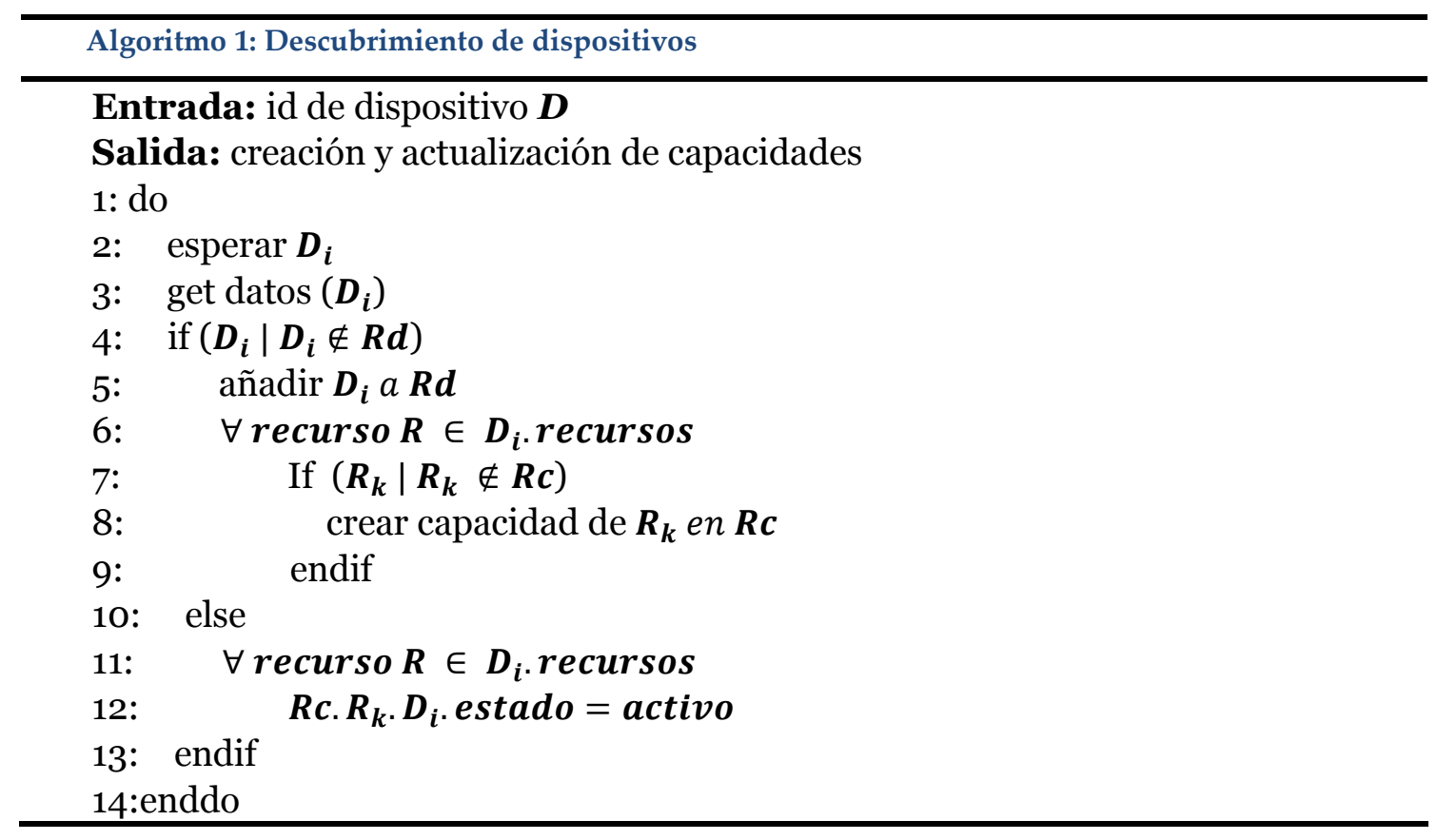

El proceso de descubrimiento permite conocer los dispositivos y los recursos, que permitirán realizar las tareas necesarias para resolver las capacidades, mientras oculta los detalles de la interfaz de comunicación con los recursos subyacentes. Esta estrategia proporciona un desacoplamiento temporal y espacial, entre las capacidades y los dispositivos. Esta característica es altamente requerida en plataformas de 
ejecución de servicios en entornos de movilidad y en contextos de WSAN con comunicaciones limitadas.

\subsection{MatchMaking de componentes}

La arquitectura propuesta considera entornos en los que el acceso a los recursos utilizados para materializar servicios, están accesibles temporalmente, provocando interrupciones en la ejecución de los mismos. La visión asíncrona y espacialmente desacoplada que brinda la arquitectura propuesta, permite una dinámica, y continua resolución de componentes en tiempo de ejecución. Los componentes son resueltos por las capacidades disponibles en las mulas, utilizando un proceso matching que toma en cuenta la información de los componentes y las capacidades dentro de un repositorio determinado. Debido a que las mulas pueden tener recursos computacionales limitados y heterogéneos, se utiliza un enfoque de matching sintáctico, simplificando el intercambio de mensajes necesarios para una continua y dinámica resolución entre los componentes y sus funcionalidades.

El proceso de matching busca y cambia las capacidades, utilizadas para resolver un componente, de forma dinámica, transparente y autónoma. Este proceso también es capaz de rehacer las pérdidas de asociaciones de componente-capacidad, siempre que sea compatible, reemplazándolas por otras capacidades disponibles. Adicionalmente, las capacidades también pueden ser sustituidas cuando se encuentre otra que ofrezca mayores niveles de funcionalidad.

\subsubsection{Pre matchmaking}

El framework de gestión alojado en la cloud, permite la creación de servicios mediante componentes. Los componentes se definen en función de las múltiples capacidades registradas en el tiempo, y proporcionadas por las entidades móviles que interactúan con la arquitectura. De esta forma el framework de desarrollo de servicios cuenta con una bitácora de capacidades que pueden utilizarse para la creación de componentes. La bitácora anteriormente citada conoce las capacidades, sin embargo, no posee ningún enlace a los dispositivos que la implementan, ya que pueden existir múltiples dispositivos que la realicen. Las capacidades permiten definir condiciones básicas y opcionales, sin embargo, estas deben ser definidas mediante un vocabulario común, que también es utilizado para describir los atributos de los recursos ofrecidos pos los dispositivos en las WSAN, por ejemplo el descrito en ("Semantic Sensor Network Ontology," n.d.).

\subsubsection{Gestión de prioridades}

La selección de la mejor capacidad para la resolución de un componente, está definida por la relación entre las condiciones del componente y las restricciones de las capacidades. El algoritmo confirma la elegibilidad de la capacidad, verificando que por lo menos debe cumplir con todas las condiciones básicas del componente, y las que cumplan con el criterio de elegibilidad se registran en una lista de elegibles. 
Las condiciones opcionales de los componentes son analizadas en función de las restricciones de las capacidades en la lista de elegibles, para determinar de forma estadística cuál de ellas cumple con la mayor cantidad de condiciones opcionales, y por tanto tendrá mayor prioridad para ser elegida. Si todas las capacidades elegibles tienen la misma cantidad de condiciones satisfechas para un componente, entonces la prioridad la tendrá la primera capacidad en la lista de elegibles.

\subsubsection{Algoritmo de MatchMaking}

Dadas las características complejas del proceso de matching y la carga computacional inherente, este algoritmo es ejecutado del lado del servidor, donde está instalado el motor de ejecución de servicios. El MM definido mediante el Algoritmo 2, tiene como objetivo principal determinar la mejor compatibilidad de las condiciones de los componentes del servicio, previamente definidas por el usuario durante la creación, y las capacidades ofrecidas por las mulas. La llamada a este algoritmo se realiza desde el algoritmo de delegación de servicios que se explica posteriormente en la sección 7.3, del capítulo 7.

El algoritmo recibe de su invocación la información de id de servicio, el componente y el id de la mula que porta el repositorio de capacidades a evaluar, es decir, la que realiza la llamada. Los componentes y capacidades deben coincidir inicialmente en el atributo "tipo", por lo tanto, para cada capacidad en el repositorio se examina dicho atributo (3). En el caso de no tener el mismo tipo, el procedimiento retorna falso, no crea ningún nexo entre componente y capacidad. Adicionalmente a la verificación de tipo, se debe asegurar que el servicio $(S)$ tienes los permisos de necesarios para utilizar capacidad $\left(C p_{i}\right)$, para ello invoca a la función acceso (4), esta función verifica los permisos al estilo Unix. Los componentes tienen definidas condiciones básicas para un correcto funcionamiento del servicio, por lo tanto, son estricto cumplimiento. El algoritmo compara sintácticamente las condiciones básicas con respecto a los atributos y las restricciones impuestas por la capacidad (7). Las capacidades $\left(\boldsymbol{C} \boldsymbol{p}_{i}\right)$ que cumplan las condiciones son agregadas a la lista de elegibles básicas (Le.básicas) (11).

Posteriormente, se comparan las condiciones opcionales del componente con respecto a los atributos y restricciones de la capacidad $\left(\boldsymbol{C} \boldsymbol{p}_{i}\right)$. Cada condición opcional que puede ser cumplida por la capacidad evaluada, incrementa una cantidad que se registra en una lista paralela a la lista de capacidades elegibles (17). Cuando se hayan evaluado todas las capacidades $\left(C \boldsymbol{p}_{i}\right)$ en el repositorio de la mula (M.Rc), se elige de la lista de elegibles básicas, aquella que cumpla con las mayor cantidad de las condiciones opcionales. Para esto se busca en la lista de elegibles opcionales (Le.opcionales) el valor más alto y se recupera el índice (26). Con este índice se referencia la capacidad, en la lista de elegibles básicas, que es la que tiene mayor compatibilidad con el componente del servicio que se quiere resolver. Cuando se ha determinado la capacidad $\left(C p_{i}\right)$ más compatible para el componente $\left(C_{i}\right)$, se crea un registro con el vínculo $\left(\boldsymbol{C}_{\boldsymbol{i}}, \boldsymbol{C} \boldsymbol{p}_{\boldsymbol{i}}\right)$ que resuelve el matching, y queda almacenado en el 
repositorio de MM (Rm). Si el matching se resuelve con éxito el algoritmo de $\mathbf{M M}$ retorna un valor booleano verdadero.

Algoritmo 2: MatchMaking

Entrada: componente $\boldsymbol{C}$ del servicio $\boldsymbol{S}$, id de servicio, id de mula

Salida: compatibilidad, resolución de componente

1:función MatchMaking $\left(\boldsymbol{C}_{\boldsymbol{i}}, \boldsymbol{S}, \boldsymbol{M}\right)$

2: $\forall$ capacidad $\boldsymbol{C p} \in \boldsymbol{M}$. $\boldsymbol{R c}$

3: $\quad$ if $\left(\boldsymbol{C}_{\boldsymbol{i}}\right.$.tipo $=\boldsymbol{C} \boldsymbol{p}_{\boldsymbol{i}}$.tipo $)$

4: $\quad$ if $\left(\operatorname{acceso}\left(\boldsymbol{S}, \boldsymbol{C} \boldsymbol{p}_{\boldsymbol{i}}\right)\right)$

5: $\quad \forall$ condición $\boldsymbol{c b} \in \boldsymbol{C}_{\boldsymbol{i}}$. básicas

6: $\quad \forall$ restricción $\boldsymbol{r t} \in \boldsymbol{C p}_{\boldsymbol{i}}$

7: $\quad$ if $\left(\boldsymbol{c} \boldsymbol{b}_{\boldsymbol{i}} \equiv \boldsymbol{r} \boldsymbol{t}_{\boldsymbol{k}}\right)$

8: $\quad$ incrementar imperativas

9: $\quad$ endif

10: $\quad$ if (imperativas $=$ cant $\left(\boldsymbol{C}_{i}\right.$. básicas $\left.)\right)$

11: $\quad$ agregar $C p_{i}$ a Le.básicas

12: $\quad \forall$ condición cop $\in C_{i}$. opcionales

13: $\quad \forall$ restricción $\boldsymbol{r t} \in \boldsymbol{C p}_{\boldsymbol{i}}$

14: $\quad$ if $\left(\operatorname{cop}_{i} \equiv r t_{k}\right)$

15: $\quad$ incrementar no_imperativas

16: $\quad$ endif

17: $\quad$ agregar no_imperativas a Le.opcionales

18: endif

19: endif

21: if ( $\neg$ empty (Le.básicas))

22: $\quad$ Mayor $=$ Le.opcionales $[1]$

23: $\quad \forall \boldsymbol{C p} \in$ Le.básicas

24: $\quad$ if (Le.opcoinales $[i]>$ Mayor $)$

25: $\quad$ Mayor $=$ Le. opcionales $[i]$

26: $\quad I d x=i$

27: $\quad$ endif

28: registrar binding $\left(\boldsymbol{C}_{\boldsymbol{i}}\right.$, Le. básicas $\left.[\boldsymbol{I d x}]\right)$ en $\boldsymbol{R m}$

29: else

30: return false

31: endif

32:return true

1: función acceso $\left(\boldsymbol{S}, \boldsymbol{C} \boldsymbol{p}_{\boldsymbol{i}}\right)$

2: if $\left(C p_{i}\right.$. Dispositivo. permisos.otros $=$ público $)$

3: return true

4: else

5: if (S.creador $=C p_{i}$. Dispositivo. permisos. dueño)

6: return true

7: else

8: $\quad$ if (S.creador $=C p_{i}$. Dispositivo. permisos. grupo)

9: $\quad$ return true

10: endif 
11: endif

12:return false

\subsection{Escenario de aplicación}

La noción de red tolerante a retardos es útil en muchos escenarios. El objetivo principal es proporcionar conectividad y servicios de red donde la infraestructura de red es mínima o inexistente, tales como desastres, ambientes rurales o remotos, comunicación espacial, redes militares, etc.

En este apartado se describe un ejemplo de servicio distribuido que evidencia los desafíos analizados anteriormente en este capítulo. El primero consiste en el descubrimiento continuo de recursos, en función de los patrones de movilidad de la mula, y de los equipos que se encuentren en el radio de comunicación a su paso. También se considera el problema de la resolución dinámica de los componentes del servicio, para cumplir con las funcionalidades requeridas por el usuario. A continuación se define un escenario, que involucra ambos desafíos para la ejecución del servicio, y algunas abstracciones para una mejor comprensión de las interacciones entre los participantes.

Existe una necesidad de adquisición de datos sobre la humedad en un sembradío de arroz. El objetivo del servicio de adquisición de datos es monitorear las condiciones de humedad del suelo en el sembradío. Si este factor está por debajo de un umbral determinado, el granjero debe tomar las medidas para iniciar el proceso de regadío, por tanto, lo obliga a trasladarse al sitio que se encuentra geográficamente alejado. Este campo está situado en una zona donde no existe infraestructura de comunicación.

La zona cuenta con una red de sensores que reportan periódicamente sus lecturas a un nodo recolector (cluster head), y este los almacena temporalmente. Este nodo recolector cuenta con interfaces de comunicación con los sensores basadas en el estándar 802.15.4 (base del protocolo ZigBee) y/o Bluetooth (entre otras tecnologías de área personal) y con otras redes basadas en IP que ofrecen acceso a Internet (Gsm, Wifi, Lte, etc.).

En la Figura 19, se puede observar el diagrama de intercambio de mensajes entre las entidades que participan del servicio. El nodo recolector escucha de forma permanente a aparición de un vecino con el que pueda comunicarse, siguiendo un paradigma de comunicación oportunista. En el momento que aparezca una mula DTN, el nodo recolector intercambia mensajes DTN's con la mula (1), y establecen un canal de comunicación de datos. La mula le solicitará los datos de sus recursos disponibles, el nodo cluster head (ch) contestará con una réplica de los datos solicitados (2). Una vez que la mula recibe esos datos, los procesa mediante el algoritmo de descubrimiento, generando unas capacidades (3) que pueden ser ejecutadas por el nodo $\boldsymbol{c h}$. Este proceso es persistente en el tiempo, de esta forma la mula descubre nuevos vecinos, que ofrecen otros recursos para generar nuevas capacidades que se registran en el repositorio de capacidades de la mula $\boldsymbol{M}$. $\boldsymbol{R} \boldsymbol{c}$. 
El servicio de monitoreo de la humedad, está compuesto por varios componentes que debe ser resueltos por las capacidades a bordo de las mulas. Cuando dicho servicio esté en ejecución, y se produzca el evento del arribo de una notificación de mula al framework de servicios vía Internet (4), se invocará el procedimiento de Match. Este procedimiento realiza invocaciones sucesivas al algoritmo de MatchMaking, quien realizará un matching sintáctico para evaluar si la mula tiene todas o algunas de las capacidades necesarias para resolver los componentes del servicio (5). Si las capacidades resuelven algún componente, se crea un enlace desacoplado entre el componente y la capacidad.

Finalmente, cuando la mula vuelve a tener contacto con ese cluster head, invocará las peticiones de información de humedad sobre el dispositivo concreto, el cual devolverá los datos solicitados (7). Estos datos serán devueltos al framework de servicios cuando la mula tenga acceso a Internet (8), quedando ejecutada la capacidad que resuelve la funcionalidad de un componente del servicio (9). Cuando todos los componentes sean resueltos (10), el servicio se ejecuta en su totalidad, y su estado se conmuta hacia finalizado.

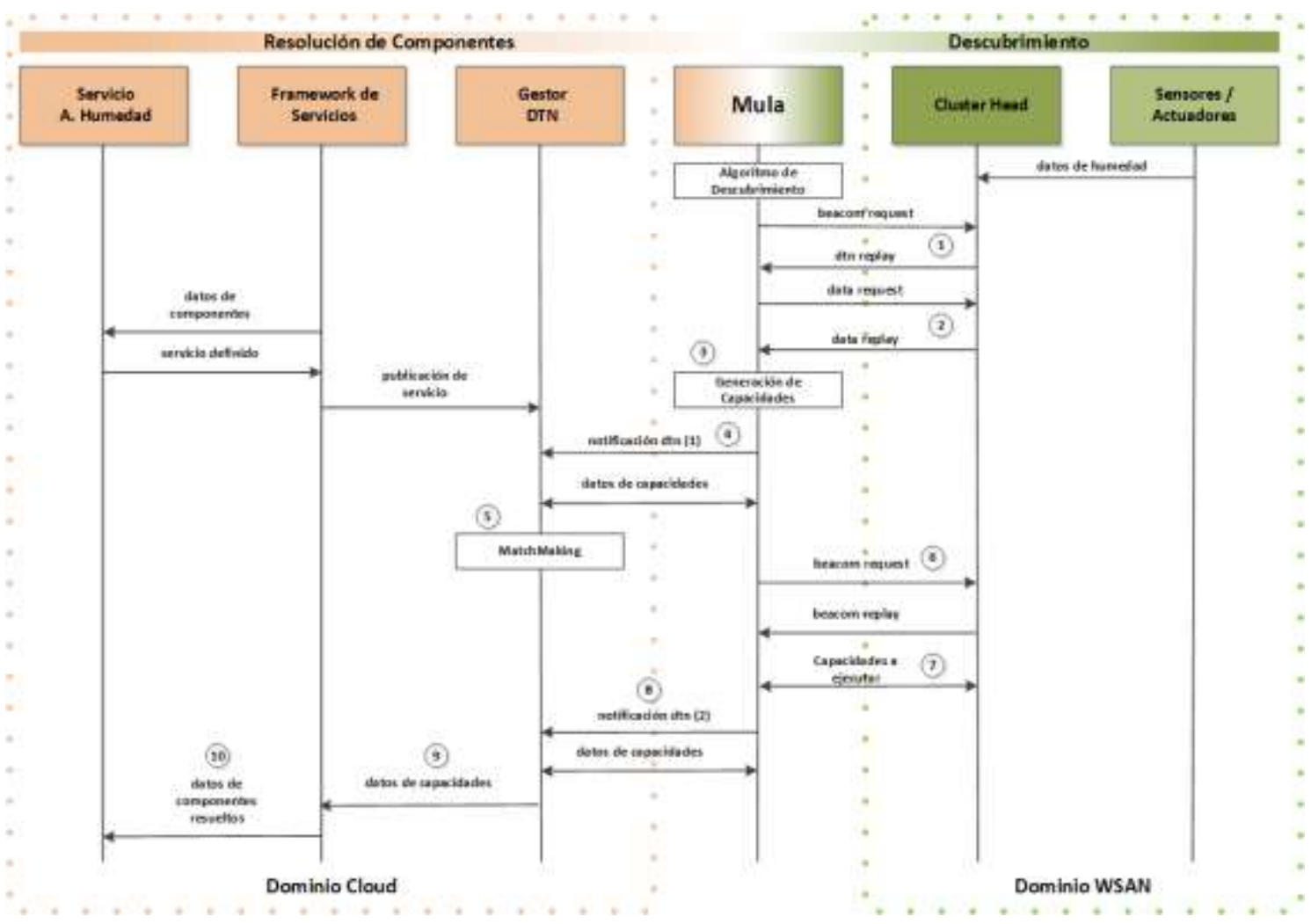

Figura 19: Resolución de componentes y descubrimiento de dispositivos 


\subsection{Conclusiones}

En este capítulo se ha propuesto una estrategia para la distribución de servicios, con el objetivo de conseguir una eficiente ejecución de los mismos, en redes inalámbricas de sensores y actuadores que disponen de infraestructura de comunicación limitada.

Se propone un modelo de ejecución de servicios que considera los desafíos de la arquitectura propuesta, ofreciendo soporte a las múltiples adaptaciones del servicio en tiempo de ejecución, producto de la heterogeneidad de los recursos tecnológicos en los entornos de WSAN y dispositivos móviles. Las principales estrategias de ejecución de servicios consisten en la resolución dinámica de los componentes, y la asociación temporal a los recursos conocidos mediante el proceso de descubrimiento.

Sobre el descubrimiento de dispositivos podemos concluir que el proceso de descubrimiento nos permite conocer los dispositivos y los recursos, que permitirán realizar las tareas necesarias para resolver las capacidades, mientras oculta los detalles de la interfaz de comunicación con los recursos subyacentes.

Sobre el proceso de matchmaking, el algoritmo propuesto determina cual es la capacidad más compatible para un componente dado y crea registros temporales con ese vínculo, almacenando estos vínculos en un repositorio de matchmaking. 


\section{Estrategia de delegación de servicios distribuidos}

\subsection{Introducción}

En esta seción se propone, describe e implementa una estrategia flexible y eficiente para la delegación y distribución servicios basados en el framework de ejecuación descrito ene la Capítulo 6, que considere los requerimientos de las redes inalámbricas de sensores/actuadores desplegadas en ambientes hostiles o remotos (Objetivo \#7).

Atendiendo a las preferencias del usuario sobre el contexto de ejecución, los servicios podrán ser ejecutados en la cloud, especialmente los que se consideren con un nivel de complejidad superior al que puedan soportar las entidades móviles en el modelo de la arquitectura propuesta. Sin embargo, en el escenario de la WSAN aisladas es de vital importancia que la ejecución de servicios pueda ser delegada a una entidad móvil con las características necesarias para ejecutarlos.

El objetivo de la delegación es que los servicios se ejecuten lo más cercano a los sujetos participantes y obtener mejor eficiencia en la ejecución, dadas las características específicas de los escenarios de WSAN con comunicaciones limitadas. Para llevar a cabo la delegación, es necesario que la arquitectura permita el desacople de los servicios, del plano de la comunicación y las tecnologías subyacentes. Adicionalmente, las mulas deben contar con un motor de ejecución de servicios integrado, que incluya un conjunto de funcionalidades básicas para la ejecución, este motor les permitirá ejecutar servicios con un nivel de complejidad básico.

\subsection{Estructura general del proceso de delegación}

En el escenario de las WSAN con comunicaciones limitadas, y según el plano físico de la arquitectura propuesta en la sección 4.5.2, los servicios pueden ser delegados a las entidades DTN móviles (mulas). De acuerdo a los patrones de movilidad e intervalos de contacto de las mulas, se consideran dos casos básicos principales. El primero toman en cuenta los patrones de movilidad con comportamiento más o menos fijo, e 
intervalos de contacto aproximadamente constantes, al cual denominamos mulas deterministas. El segundo caso, considera la situación en que las mulas no tienen la conducta anteriormente descrita, muy por el contrario, presentan características de movilidad inestable, y patrones de temporalidad de contacto variables. En este sentido, nos referiremos a este caso como mulas indeterministas. La clasificación de las mulas en términos de lo anteriormente planteado, no tiene carácter permanente, más bien está dado en función de sus patrones de conducta. Esta identificación no es estática en el tiempo, ambas pueden pasar de una clasificación a otra en cualquier momento.

En la Figura 20, se expone la organización general del proceso de delegación. En la sección izquierda se puede observar el flujograma del algoritmo de delegación, que se describe posteriormente (Algoritmo 3). En el lado derecho se encuentran los distintos algoritmos o módulos que son invocados desde el algoritmo principal de delegación, y las interacciones entre ellos. Las relaciones entre los algoritmos se reflejan en el diagrama mediante las invocaciones lineales dirigidas. El proceso de delegación es un algoritmo cíclico permanente, este se mantiene escuchando las mulas constantemente. Ante un reporte de una mula, la atiende y luego continúa a la escucha nuevamente. El procedimiento básico consiste de determinar su clasificación y luego invocar los algoritmos de delegación indeterminista y determinista, que son explicados en las secciones 7.3.1 y 7.3.2, respectivamente.

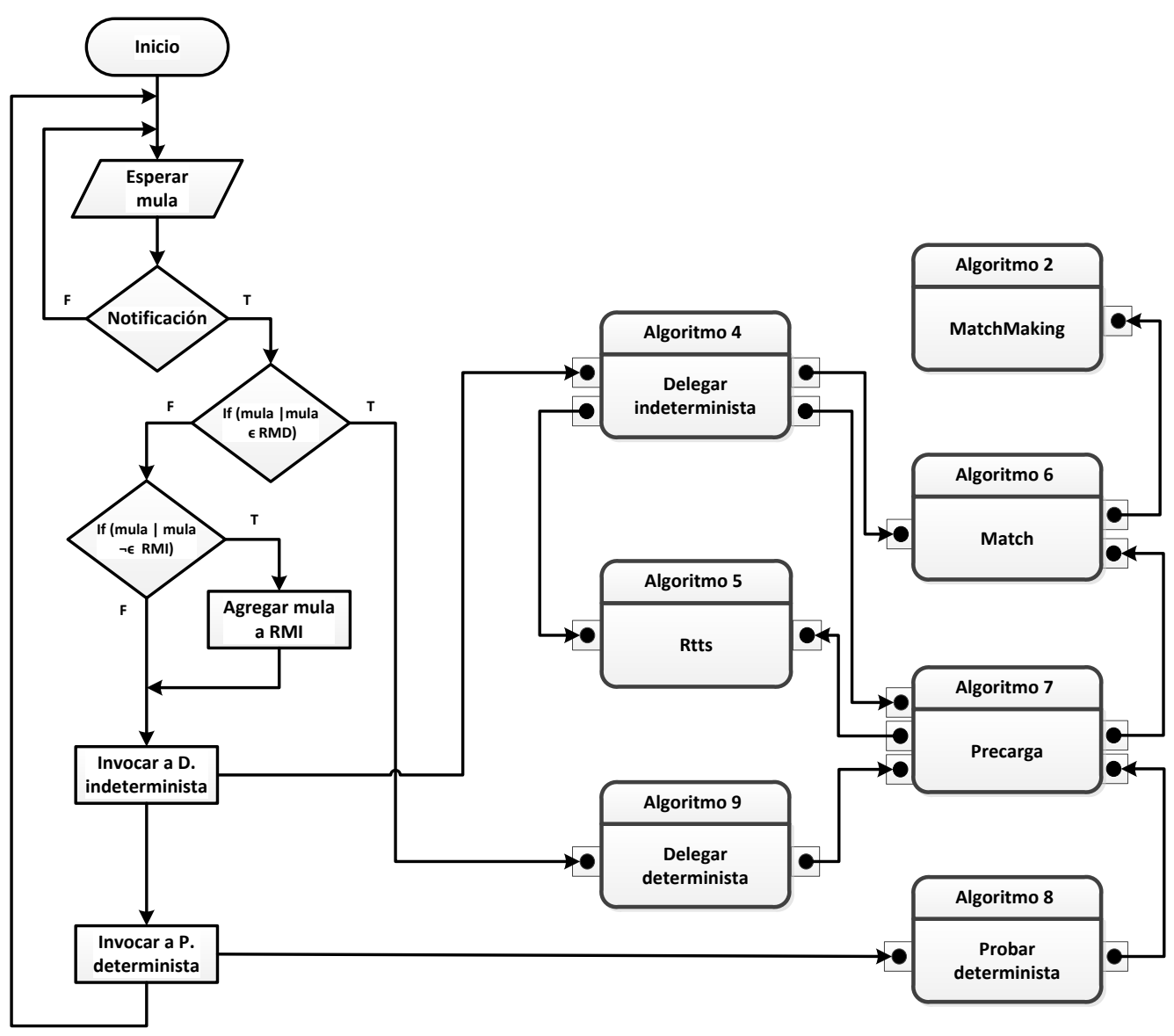

Figura 20: Esquema general del algoritmo de delegación 
El algoritmo de delegación a priori considera a todas las mulas como indeterministas, y en tiempo de ejecución ellas pueden llegar a convertirse en determinista, si satisfacen las condiciones para ser mula determinista. Cuando se resuelve la clasificación de una mula, esta se registra en un repositorio específico de cada tipo, es decir, existe un repositorio de mulas deterministas (RMD) e indeterministas (RMI). Cada vez que una mula indeterminista se notifica con el framework de servicios se computa, y se realiza la comprobación de mula determinista mediante el algoritmo "Probar determinista", que se explica en la sección 7.3.1.

La delegación de servicios se lleva a cabo cuando existe una mula DTN conectada a la infraestructura de red con acceso a la cloud, y registrada en el framework de desarrollo de servicios. El registro de las entidades DTN móviles participantes es un proceso previo a la delegación, y se asume que ha sido realizado por parte de los usuarios. Para cada equipo DTN móvil registrado y clasificado como determinista, se crea una lista de servicios de precarga ordenados por prioridad, este proceso se describe en la sección 7.3.1 con el Algoritmo 7.

Las mulas indeterministas se consideran menos confiables que las deterministas, por lo tanto, no tienen lista de precarga. Sin embargo, el algoritmo que clasifica las mulas, utiliza una variable que representa la cantidad de notificaciones al framework de servicios, para que una mula sea considerada como determinista. Este parámetro es establecido por el usuario en tiempo de configuración del servicio. Adicionalmente, el algoritmo también toma en cuenta la desviación típica de los tiempos de contacto en función de un intervalo de confianza definido por el usuario en la configuración del servicio.

El proceso de delegación indeterminista, que se define según el Algoritmo 4, intentará delegar la mayor cantidad de servicios en las listas de precarga de las mulas en el RMD. Para asegurar la correcta ejecución del servicio delegado, el algoritmo de delegación indeterminista considera otros aspectos importantes a saber; Round Trip Time Service (Rtts), descrito en la sección 7.3.1 (Algoritmo 5). Otro factor importante, es el proceso de matching (Match), ilustrado en la sección 7.3.1 (Algoritmo 6). El otro criterio contemplado es el de confianza sobre la mula, y se define en términos del factor de confianza. El cálculo del factor de confianza de una mula (M.Fc), está definido en la función "Probar determinista" (Algoritmo 8). Adicionalmente, se contempla la disponibilidad de recursos en la mula, en términos de la memoria de almacenamiento.

La delegación determinista de servicios permite confiar la ejecución de servicios a las mulas que hayan sido clasificadas y registrada en el $R M D$, este tipo de delegación se detalla en la sección 7.3.2 (Algoritmo 9). El algoritmo de delegación determinista verifica si los recursos de almacenamiento de la mula están disponibles en su totalidad, si esta condición se cumple, intentará pasar la lista completa de servicios de su lista de precarga. En caso contrario, examina los servicios individuales de su lista de precarga, con respecto a los están a bordo de la mula. Si encuentra un servicio en su lista de precarga con mayor prioridad, y tienen espacio para alojarlo, intercambia dichos 
servicios. En la siguiente sección se describen los algoritmos específicos con mayor detalle.

\subsection{Algoritmo de delegación}

El desarrollo de la delegación de servicios, se lleva a cabo como se ilustra en el Algoritmo 3. Este código es ejecutado de forma permanente por el framework de servicios, del lado de la cloud. La delegación de servicios se realiza teniendo en cuenta la clasificación de las mulas, es decir, existe un procedimiento diferente en cada caso.

Este algoritmo se activa en cuanto exista una notificación de una mula, es decir, cuando aparezca una mula participante, esta se procesa y continúa a la espera de la siguiente. En primera instancia el algoritmo realiza una petición de información a la mula (3). Es necesario determinar si la mula que se ha reportado, está registrada en el $R M D$, en caso afirmativo, se invoca a la función de "Delegar determinista" (5). En caso contrario, se examina el repositorio de mulas indeterministas (RMI) en busca del registro (7), si no se encuentra la mula se registra (8). Luego se realiza la invocación a la función "Delegar indeterminista", que ejecuta el proceso de delegación a mulas indeterministas (10). Finalmente el algoritmo, invoca la función probar determinista (11), que determinará si la mula cambia de clasificación o se mantiene. La función "Probar determinista", está definida en detalle posteriormente en el Algoritmo 8.

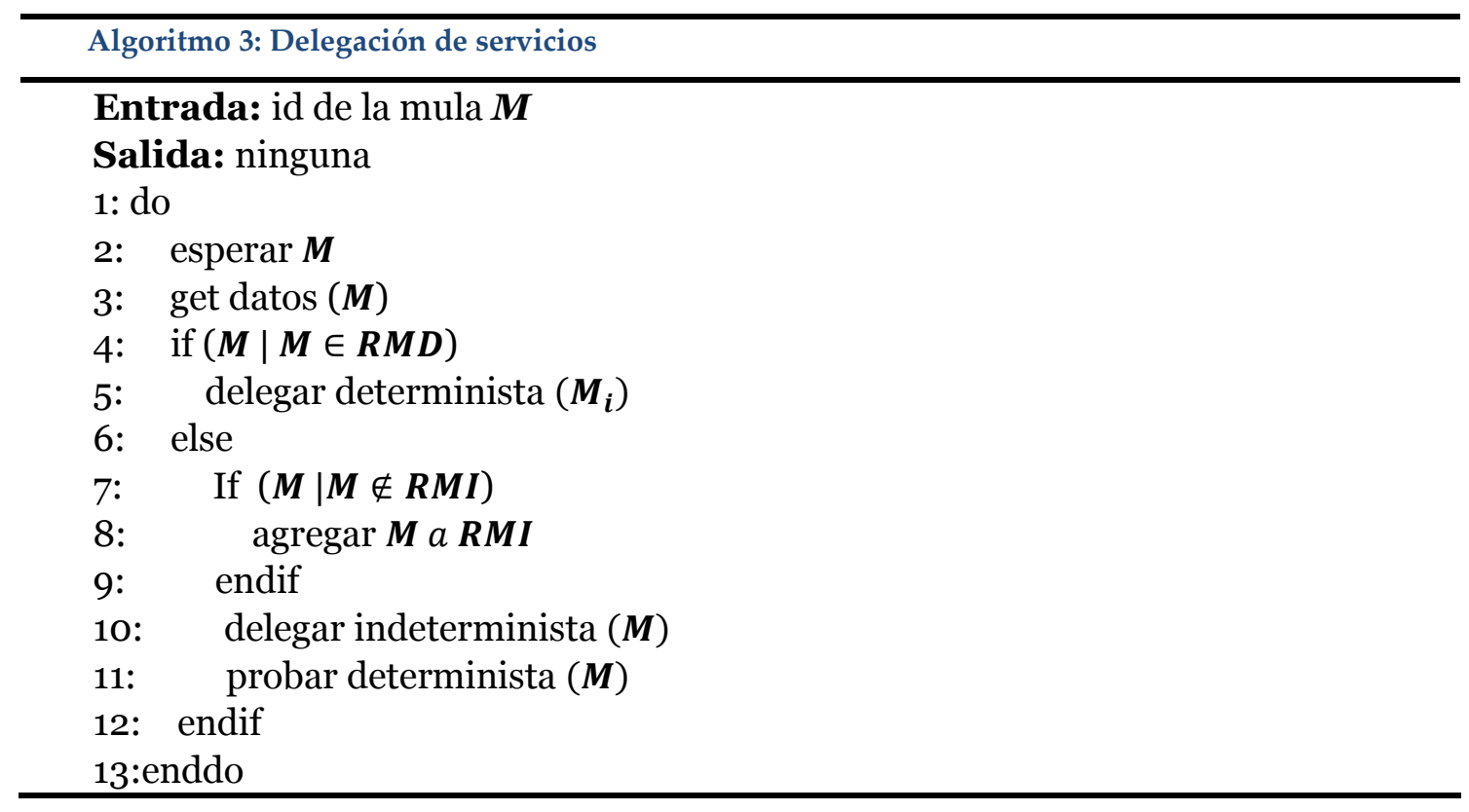

\subsubsection{Delegación indeterminista}

En el Algoritmo 4 se muestra la función de delegación indeterminista. El objetivo de esta función es delegar la mayor cantidad de servicios que se encuentran en las listas de precarga $(\boldsymbol{L p})$ de las mulas en el repositorio de mulas deterministas. El algoritmo verifica que el $\boldsymbol{R} \boldsymbol{M} \boldsymbol{D}$ no esté vació, esto significa que ya existen mulas 
deterministas $(\boldsymbol{M D})$. La lista de precarga de cada una de ellas $\left(\boldsymbol{M}_{\boldsymbol{i}} \cdot \boldsymbol{L} \boldsymbol{p}\right)$ se examina servicio por servicio $\left(\boldsymbol{S}_{\boldsymbol{k}}\right)$, para determinar si alguno de ellos puede ser delegado a la mula disponible (3-5).

Para que la delegación de servicios sea correcta, se requieren algunas comprobaciones muy importantes. La primera consiste en la verificación del tiempo de ejecución del servicio, es decir, la invocación a la función Rtts (6) que realiza el cálculo de Round Trip Time Service (Algoritmo 5). La segunda comprobación se realiza con la invocación a la función Match (6) (Algoritmo 6), que a su vez realiza invocaciones al proceso de $M M$ (Algoritmo 2), para resolver el proceso de matching. Ambas comprobaciones deben ser satisfechas positivamente.

Luego se evalúa el factor de confianza del servicio $\left(\boldsymbol{S}_{\boldsymbol{k}} . \boldsymbol{F c}\right)$ y de la mula (mula.Fc) (7). El factor de confianza está definido como $\sum_{\boldsymbol{i}} \boldsymbol{M}$. $\boldsymbol{c o n t a c t o s}_{\boldsymbol{i}} / \boldsymbol{N}$, donde $\boldsymbol{N}$ es una variable definida por el usuario. Este parámetro indica la cantidad de contactos que tiene que acumular la mula, para que sea de la confianza del usuario ( $\boldsymbol{N}=\mathbf{0}$ : no confiable; $\boldsymbol{N} \geq \mathbf{1}$ : confiable). En principio todas las mulas tienen un factor de confianza igual a cero. Cada vez que contactan con la infraestructura de servicios de calcula nuevamente este valor, mediante la función "Probar determinista". Finalmente, el algoritmo examina los recursos de almacenamiento de la mula, con respecto a los necesarios para alojar el servicio sujeto de delegación (7). En caso de que todas las condiciones han sido satisfechas, el servicio es delegado a la mula actual (8), se actualiza el estado del servicio $\left(\boldsymbol{S}_{\boldsymbol{k}}\right)$ y se recalcula la lista de precarga para la mula $\boldsymbol{M}_{\boldsymbol{i}}$ en el repositorio $\boldsymbol{R} \boldsymbol{M D}(14)$.

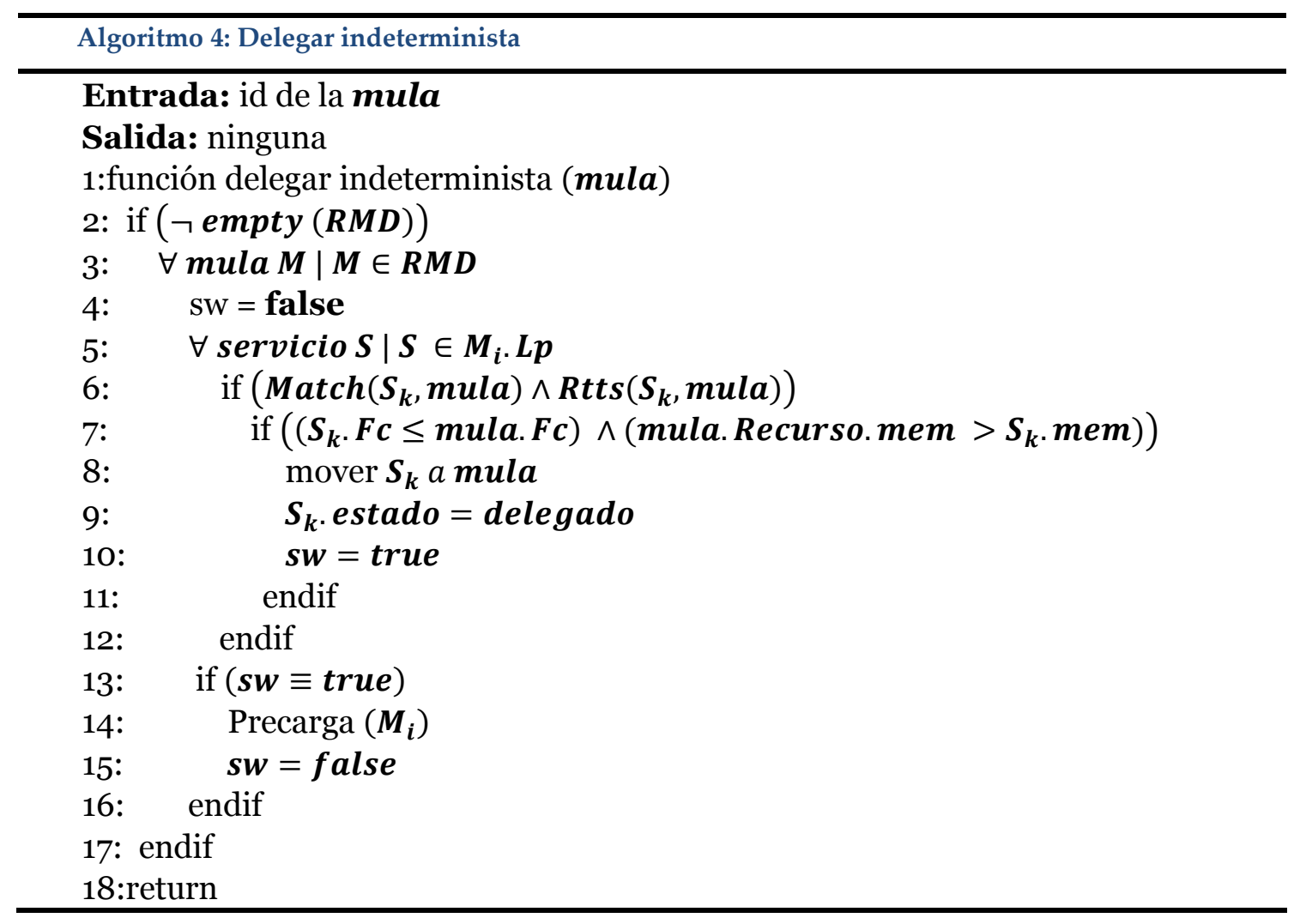


El procedimiento Rtts (tiempo de ida y vuelta del servicio), calcula cuanto tiempo aproximado pueden tomar las comunicaciones necesarias para la ejecución de un servicio específico (Algoritmo 5) en redes DTN. Se consideran los intercambios de mensajes entre tres entidades principales, que incluyen a la cloud, la mula y los dispositivos. Tomando en consideración que por cada bundle DTN enviado se esperará una confirmación de reconocimiento (ACK) por parte del receptor, el modelo estima el tiempo de ida y vuelta de cada mensaje (Yu et al., 2016). El margen para contabilizar el procesamiento, los retrasos en las colas y el tiempo de transmisión en ambos extremos es $\mathbf{2}^{\boldsymbol{N}}$ (2). Donde $\boldsymbol{N}$ es un parámetro establecido por la gestión de red, para el cual 2 segundos parecen ser un valor predeterminado razonable (Farrell, Ramadas, \& Burleigh, 2008).

El tiempo $\boldsymbol{t}_{\boldsymbol{f}}$, describe el tiempo de comunicación de del framework de servicios con una mula, y $\boldsymbol{t}_{\boldsymbol{d}}$ define el tiempo que puede tardar la mula en contactar con cluster head que coordina los sensores/actuadores necesarios para realizar tareas requeridas por el servicio. Estos valores se calculan como el promedio estadístico, a partir de los registros históricos de contactos de las mulas con los dispositivos, estos valores se actualizan en función de los eventos de contactos. Estos metadatos se calculan para cada componente $\boldsymbol{C o}_{\boldsymbol{i}}$ del servicio $\boldsymbol{s}$ (3), y por cada par componente-capacidad $\left(\boldsymbol{C o} \boldsymbol{o}_{\boldsymbol{i}}, \boldsymbol{C} \boldsymbol{p}_{\boldsymbol{k}}\right)$, que se encuentre registrada en $\boldsymbol{R m}$ (5).

El tiempo de comunicación con el framework (tcff), define el tiempo de comunicación que tardaría en servicio en ejecutarse desde la cloud (8). El otro criterio de temporalidad es el tiempo de comunicación con la mula (tcm), el cual considera el tiempo de ejecución del servicio si este es delegado a la mula (6). Este valor se compara con el valor máximo de ejecución del servicio s.tmax, que es definido por el usuario en tiempo de creación (9), para determinar si ese servicio es elegible en tiempo, para ser delegado a la mula.

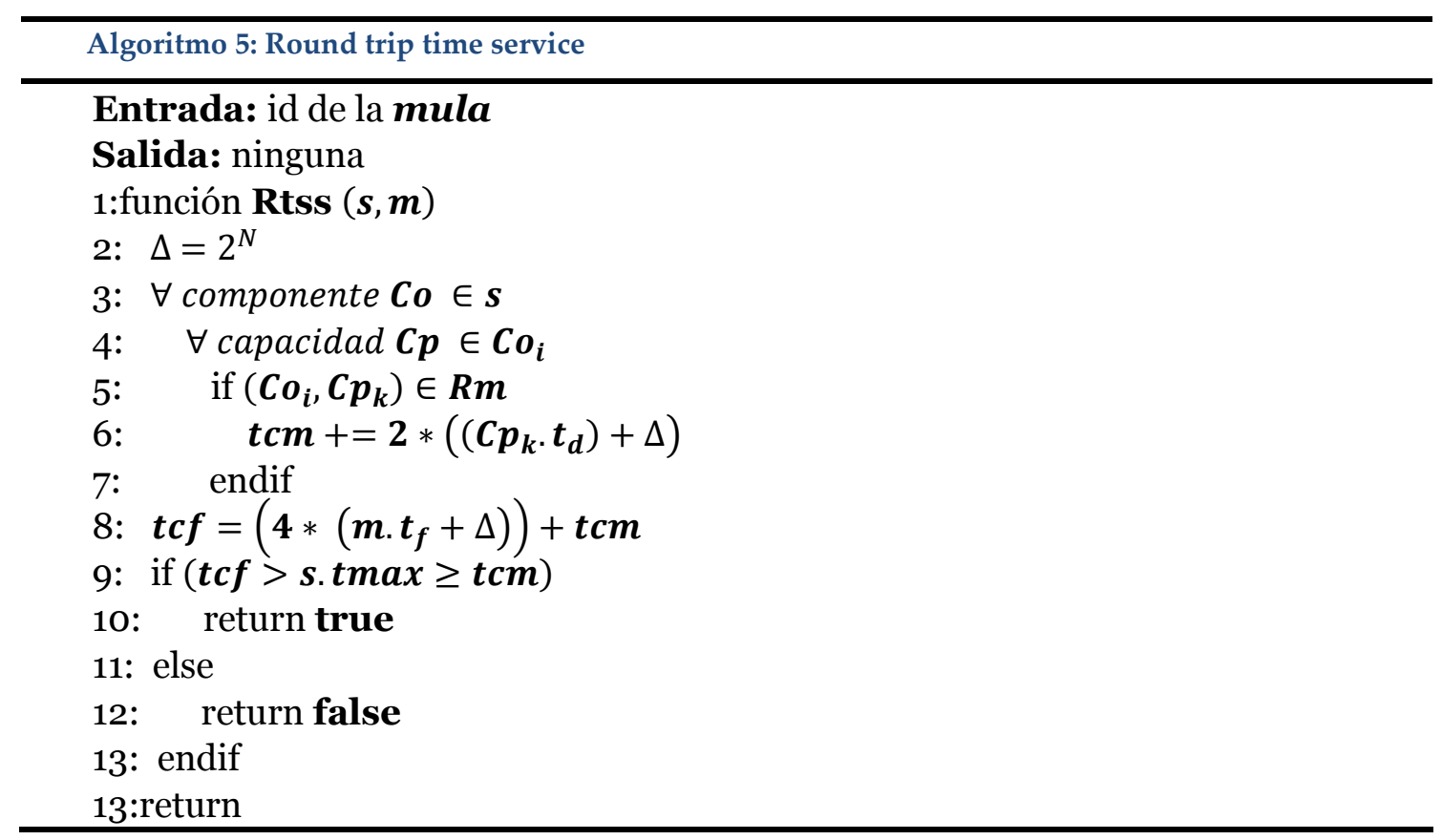


El Algoritmo 6 ilustra la función Match, esta utiliza un método que examina cada componente $(\boldsymbol{C})$ del servicio ( $\boldsymbol{s}$ ) (2) con respecto a las capacidades de la mula ( $\boldsymbol{m}$ ), realizando invocaciones sucesivas al algoritmo de $M M(3)$, descrito anteriormente en el capítulo 6. Esta función devuelve valores booleanos con respecto al proceso de matching, para cada componente del servicio que se está evaluado.

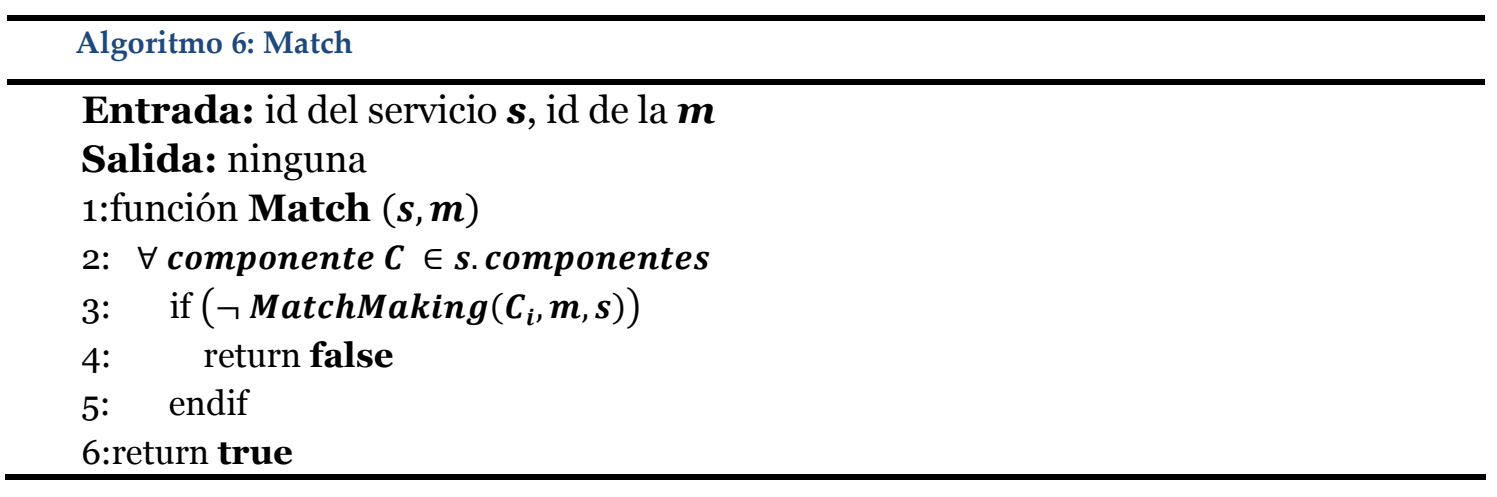

La función de precarga se muestra en con el Algoritmo 7. La importancia de esta función es que permite traspasar lo más rápido posible los servicios compatibles con una mula específica, cuando esta se notifique. La precarga tiene como objetivo buscar en el repositorio de servicios $\boldsymbol{R} \boldsymbol{s}$, los servicios $\boldsymbol{S}_{\boldsymbol{i}}(2)$ que pueden ser delegados a una mula determinada, creando con ello una lista de precarga. Para la creación de la listas de precarga el algoritmo toma en cuenta dos factores importantes (3), el primero es el proceso de matching, y el segundo es el cálculo de Rtts, ambos descritos con anterioridad.

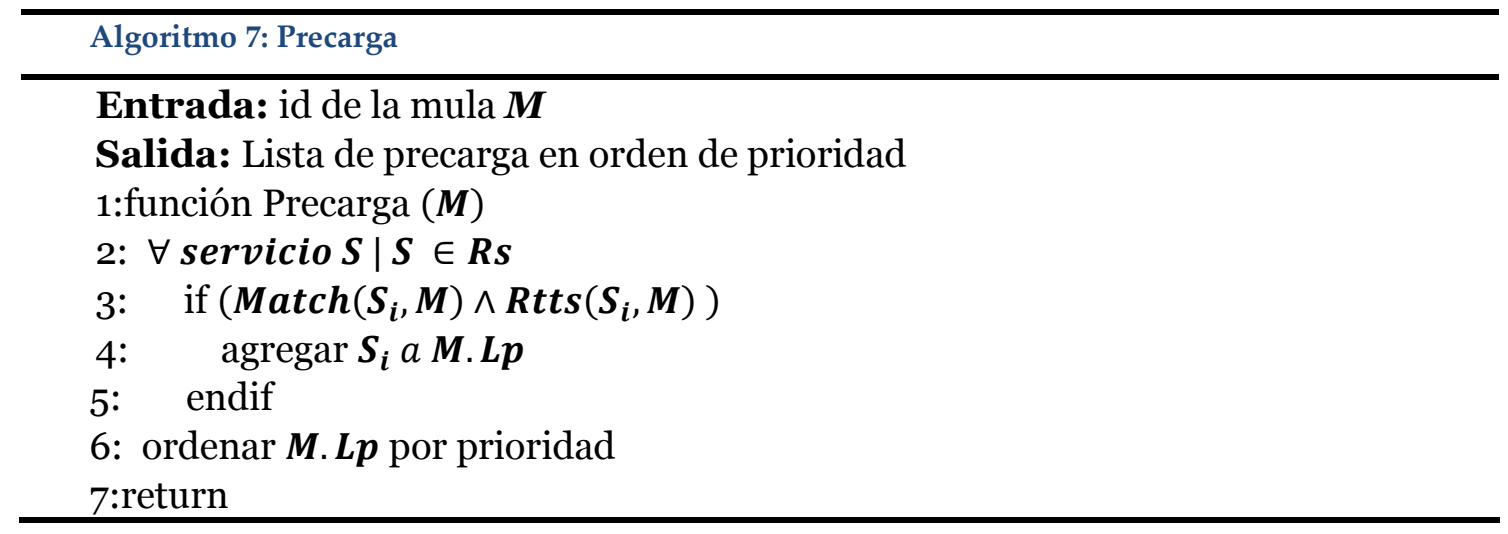

El Algoritmo 8, implementa la función "Probar determinista", que resuelve dinámicamente la clasificación de las mulas. Esta función se invoca desde el algoritmo de delegación de servicio, cada vez que hay una delegación indeterminista. Este algoritmo computa la cantidad de contactos con el framework de servicios. También, registra el tiempo entre contactos (retardos), y con ellos se crea una lista de retardos (M.Lr). Se computa el promedio de retardos de la lista $(\overline{\boldsymbol{R}})(6)$, para calcular la 
desviación típica de los retardos (S) (7). El factor $\boldsymbol{X}$ representa un rango de precisión aceptable para los valores de las desviaciones de los retardos, este parámetro es definido por el usuario en la creación del servicio. Luego se evalúa que si la desviación $\boldsymbol{S}$, está dentro del rango de precisión $\boldsymbol{X}(8)$, se calcula el factor de confianza de la mula (M.F C) (9), que está dado por la cantidad de contactos de la mula dividido entre $\boldsymbol{N}$, que representan el número de contactos que usuario ha definido, para confiar en la mula. Si las condiciones son satisfechas por la mula, esta se elimina de $\boldsymbol{R} \boldsymbol{M I}$ y se agrega en el $\boldsymbol{R M D}$. Finalmente, se invoca la función Precarga para generar la lista de precarga de $M$.

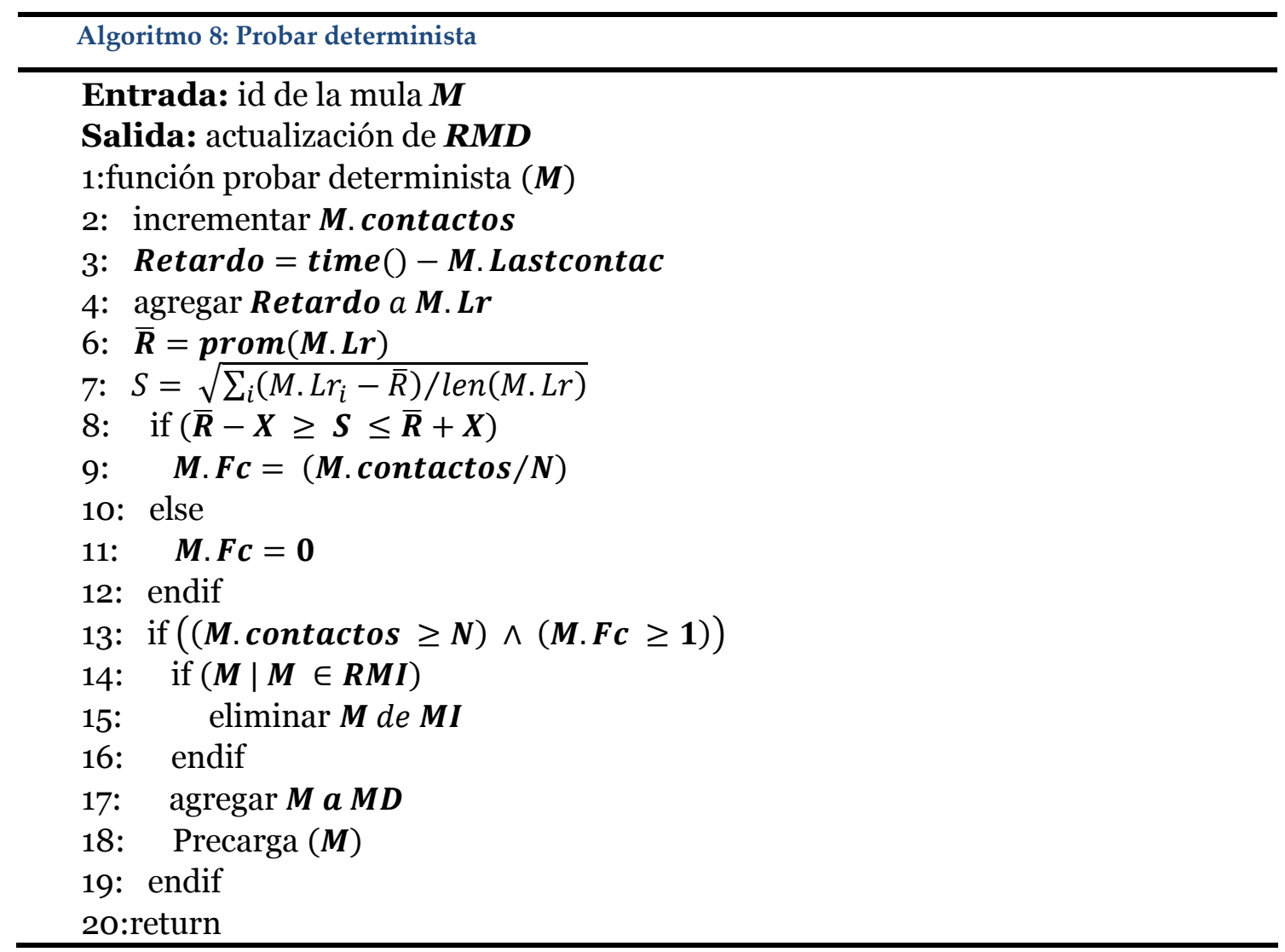

\subsubsection{Delegación determinista}

El método utilizado para para procesar la delegación hacia mulas deterministas, se puede observar en el Algoritmo 9. La idea fundamental es aprovechar el procesamiento a priori de las listas de precarga, para mejorar la eficiencia en el despacho de los servicios a la mula.

Este algoritmo contempla dos situaciones, una es cuando se reporta una mula cuya lista de carga (mula. Lc) está vacía. En esta situación, el framework intentará pasar la lista de precarga (M. $\boldsymbol{L} \boldsymbol{p}$ ) completa hacia la mula. Sin embargo, el espacio de memoria disponible en la mula debe ser suficiente para alojar todos los servicios en la lista de precarga de la mula $\left(\sum_{i}^{n} \boldsymbol{M} . \mathbf{L} \boldsymbol{p} . \boldsymbol{S}_{\boldsymbol{i}} . \mathbf{m e m}\right)$. Si estas condiciones se satisfacen, se procede 
a mover los servicios a la mula (4) y luego se actualiza el estado de los servicios delegados (5). En este caso concreto se debe realizar una precarga de la mula $\boldsymbol{M}$, con el objetivo de regenerar una nueva M. $\boldsymbol{L} \boldsymbol{p}(6)$.

El segundo caso se presenta cuando la mula que se reporta, lleva a bordo una carga de trabajo, es decir una lista de carga (mula. $\mathbf{L c}$ ). En esta situación el framework utiliza una alternativa distinta. El algoritmo intenta delegar los servicios de la lista de precarga de la mula ( $\boldsymbol{S} \boldsymbol{p} \in \boldsymbol{M} . \boldsymbol{L p})$ (9). El criterio utilizado en primera instancia está dado en función de la prioridad, es decir, si un servicio en la lista de precarga tiene mayor prioridad que alguno de los servicios a bordo de la mula, y que además, su estado es diferente de ejecución (11), se convierte en candidato a delegación. La delegación se autoriza cuando la memoria necesaria para el servicio a delegar, debe ser menor o igual a la memoria necesaria para almacenar el servicio de menor prioridad en la lista de carga de la mula (12). En este caso se lleva a cabo la delegación del servicio, se mueve el servicio de la lista de carga $\left(\boldsymbol{S} \boldsymbol{c}_{\boldsymbol{k}}\right)$ hacia el repositorio de servicios $(\boldsymbol{R} \boldsymbol{s})$. Luego se mueve el servicio a ser delegado $\left(\boldsymbol{S} \boldsymbol{p}_{\boldsymbol{i}}\right)$ hacia la mula y se actualiza su estado. Finalmente se actualiza la lista de precarga de la mula invocando a la función "Precarga" (18).

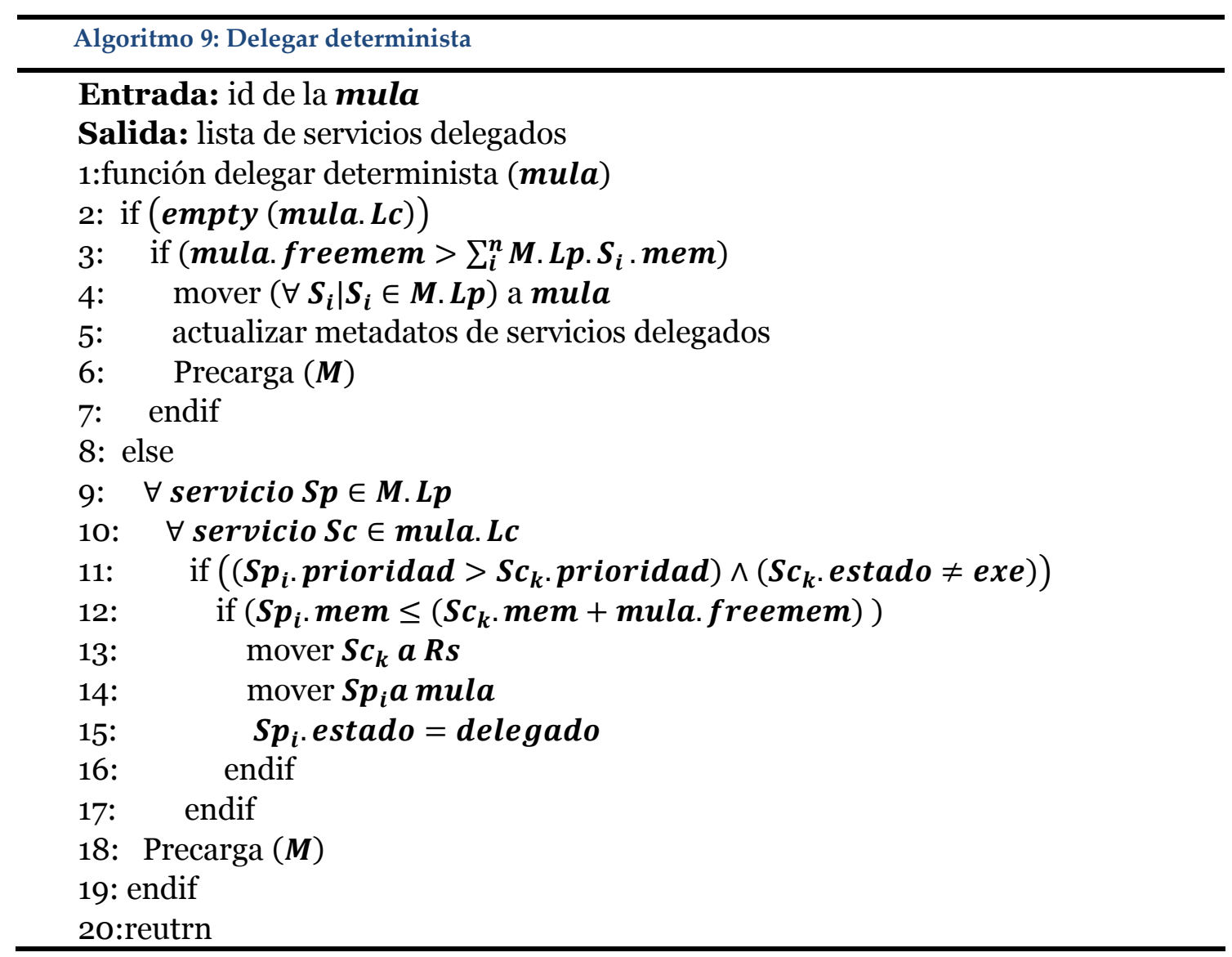




\subsection{Escenario de aplicación}

En esta sección se describe un escenario basado en una granja inteligente de cultivos. Esta parcela utiliza una arquitectura de IoT de bajo costo, para la monitorización y automatización de tareas propias de los cultivos en zonas con sistemas de regadío artificial. La granja está en una posición geográfica que no cuenta con infraestructura de telecomunicaciones que ofrezca servicio de acceso con redes externas (Internet).

En la Figura 21, se pueden observar los actores principales en este contexto de aplicación. El campo de cultivos cuenta con una red de sensores que permiten medir algunas propiedades del ambiente como temperatura, humedad del suelo, alcalinidad, saturación de pesticidas, etc. (1). Esta información fluye de los sensores individuales hasta el cluster head (2) de la de la WSAN. Con esta información se toman las decisiones sobre las siguientes tareas a realizar en torno a optimizar la producción.

Una de las acciones a seguir puede ser la irrigación, como consecuencia de sobrepasar el umbral hídrico mínimo en el suelo. Esta tarea es realizada por actuadores, como por ejemplo una bomba de agua instalada en un río, a unos cuantos kilómetros de distancia (4). Esta bomba cuenta con un cluster head (3) que le permite recibir instrucciones de operación, sin embargo, este no tiene comunicación directa con la red de sensores de la parcela. En ambos casos los nodos coordinadores de las WSAN cuentan con dual stack de comunicaciones, que les permite comunicarse con los sensores/actuadores (Bluetooth, ZigBee, Xbee, etc.) y además, también puede comunicarse con otros equipos (buses, carros u otros), a través de otras interfaces de comunicación inalámbrica de banda ancha (Wi-fi, Gprs, WiMax, Lte, etc.).

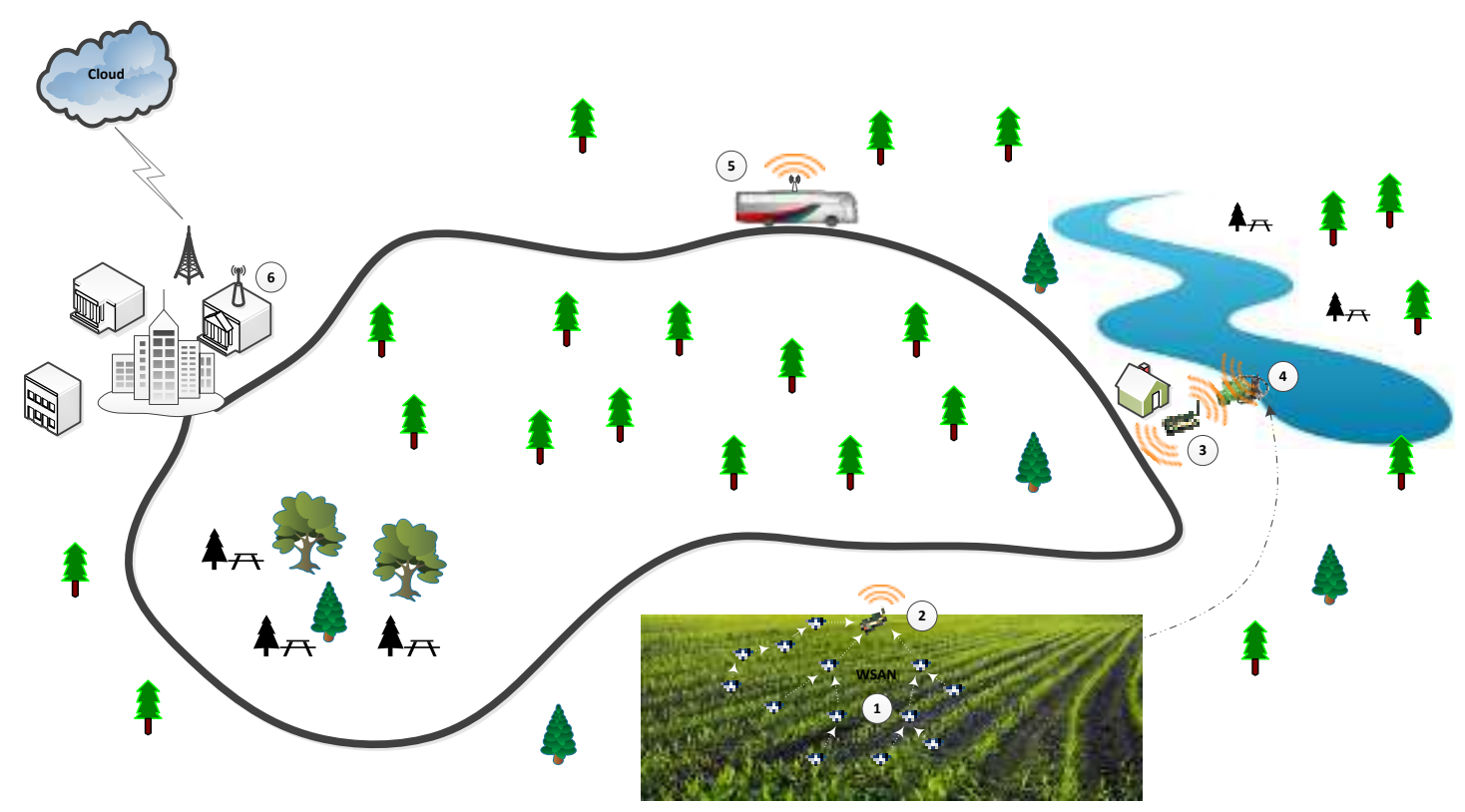

Figura 21: Escenario de granja inteligente 
El campo de cultivos y la estación de regadío, se encuentran cercanos a carreteras circundantes, donde existen rutas de transporte de buses y automóviles. Los buses circulan por la ruta a intervalos de tiempo regulares, estos cuentan con una estación de autobuses en la ciudad, la cual proporciona acceso a Internet (6). La línea de buses que circula por esta ruta, llevan equipos a bordo que cuentan con interfaces de comunicación inalámbricas, que le permiten intercambiar información con los nodos cluster head en el área de cobertura de la señal de radio (5). Esta información es almacenada, hasta que encuentra una alternativa de envío, es decir, utilizan un paradigma de comunicación oportunista, con una estrategia de almacenamiento, transporte y envío.

Las oportunidades de comunicación de los cluster head con las mulas (buses o automóviles) son muy volátiles, y dependen de la velocidad de la mula, rangos de comunicación, velocidad de procesamiento, entre otros. Lo anterior indica que es muy probable que la información no sea transmita completamente en esa ventana de tiempo, como consecuencia de las comunicaciones intermitentes o disruptivas. Los datos que deben ser transmitidos pueden ser objeto de fragmentaciones, producto de las características de movilidad de la mula. Esto trae como consecuencia la retransmisión de todos los datos nuevamente, además, de la pérdida de la oportunidad de comunicación. En este entorno las comunicaciones se inician antes de que establezca una ruta de comunicación de extremo-a-extremo, y los retardos pueden alcanzar umbrales que los protocolos como TCP son incapaces de tolerar. Las funcionalidades de las DTN, ofrecen una alternativa ante tales requerimientos.

En este escenario el granjero de forma remota, puede crear un servicio para activar el regadío de la parcela. Atendiendo al contexto de ejecución del servicio, este puede ejecutarse de dos formas. La primera alternativa es que se ejecute directamente en la cloud, en cuyo caso los eventos tendrían la siguiente secuencia: adquisición de los datos de los sensores, transporte hasta la cloud, procesamiento, posteriormente se envía el la orden de regadío, que tiene que ser transportada hasta la bomba de agua para poder completar el servicio. La segunda opción, consiste en delegar el servicio hacia la mula. Es ente caso el servicio tendría la siguiente secuencia de eventos: delegación del servicio a la mula, obtención de los datos de la parcela, procesamiento a bordo de la mula, y luego el envío de la información de los detalles del regadío hacia la bomba de agua.

En este escenario, y conociendo las circunstancias expuestas anteriormente, la delegación del servicio puede ser una buena alternativa, dado que permite que los servicios se ejecuten más cerca de los participantes, optimizando de la ejecución de los servicios en estos entornos. El algoritmo de delegación está basado en el criterio de determinismo de la mula, que está descrito en el Algoritmo 8. Este concepto intenta optimizar la transferencia de los servicios a la mula, durante el tiempo de delegación de los servicios. En la delegación indeterminista (Algoritmo 4), se prevé mayor consumo de tiempo producto de una mayor complejidad en el proceso de delegación (cálculos intensivos “( $\left.\left.\boldsymbol{R t t s}\left(\boldsymbol{S}_{\boldsymbol{k}}, \boldsymbol{m u l a}\right) \wedge \boldsymbol{M a t c h}\left(\boldsymbol{S}_{\boldsymbol{k}}, \boldsymbol{m u l a}\right)\right) "\right)$. Por su parte, la 
delegación determinista (Algoritmo 9), planifica mediante un procesamiento previo (listas de precarga $\forall$ servicio $\boldsymbol{S p} \in \boldsymbol{M} . \boldsymbol{L p}$ ) al contacto de una mula determinista, permitiendo la optimización de los tiempos de delegación. En ambos casos los algoritmos consideran el criterio de prioridad del servicio (los de mayor prioridad se atienden primero " $\left(\left(\boldsymbol{S} \boldsymbol{p}_{\boldsymbol{i}}\right.\right.$. prioridad $>\boldsymbol{S} \boldsymbol{c}_{\boldsymbol{k}}$. prioridad $\left.) \wedge\left(\boldsymbol{S} \boldsymbol{c}_{\boldsymbol{k}} \cdot \boldsymbol{e s t a d o} \neq \boldsymbol{e x e}\right)\right)$ "), el cual permite brindar un nivel de granularidad en la calidad de servicio. Adicionalmente, los algoritmos toman en cuenta la disponibilidad de recursos de la mula ("(mula.freemem $\left.>\sum_{i}^{n} \boldsymbol{M} . \mathbf{L p} . \boldsymbol{S}_{\boldsymbol{i}} . \mathbf{m e m}\right)$ "), ya que los mismos cambian dinámicamente durante en el tiempo de ejecución de los servicios.

\subsection{Conclusiones}

Esta sección tiene el objetivo de proporcionar mecanismos para que la ejecución de servicios pueda ser delegada a una entidad móvil con las características necesarias para ejecutarlos.

El proceso de delegación se describe con detenimiento sobre un esquema general. Se distingue entre delegación indeterminista y determinista. La delegación indeterminista ocurre cuando las mulas presentan características de movilidad inestable, y patrones de temporalidad de contacto variables. La delegación determinista cuando las mulas presentan patrones de movilidad con comportamiento más o menos fijo, e intervalos de contacto aproximadamente constantes. Para cada tipo de delegación se presenta el algoritmo que regula su comportamiento.

Finalmente se presenta un escenario de aplicación basado en una granja inteligente de cultivos donde se demuestra como el algoritmo de delegación permite que los servicios se ejecuten más cerca de los participantes, optimizando de la ejecución de los servicios en estos entornos. 


\section{Ejecución de servicios en redes DTN}

\subsection{Introducción}

En esta sección propongo una estrategia de ejecución de servicios a bajo nivel sobre nodos en redes de sensores y actuadores (Objetivo \#8), de forma que se contribuya con una solución de gestión de servicios a bajo nivel, para la asociación física de una ejecución de servicios con un dispositivo hardware. Se asume que dichas redes de sensores y actuadores están en sitios aislados, y sus comunicaciones son limitadas, como se han definido en la capa más baja del escenario de la Sección 4.3.

En las anteriores contribuciones de este trabajo de tesis no se describe la fase final de ejecución, donde un dispositivo concreto es seleccionado para realizar una acción. De esta forma, en esta sección se describe una solución relacionado con la gestión de servicios para la coordinación de la ejecución en nodos de la red de sensores y actuadores. Esta solución emplea una técnica de selección probabilística basada en el concepto de Coste y Calidad de Servicio (QoS), incluyendo dos procedimientos de orquestación y coreografía y un análisis e su rendimiento que se detallará en la Sección 9.

\subsection{Ejecución de servicios en redes DTN}

En escenarios reales, los dispositivos hardware presentan una gran heterogeneidad e interfaces propietarias. Esto da lugar a dispositivos que realizan las mismas funciones pero que responden a distintos lenguajes, protocolos, o modelos de datos. Además, la necesidad de ubicuidad del entorno presentado en este trabajo de Tesis requiere soluciones para la gestión de la ejecución de servicios a bajo nivel, de forma que se recolecte información sobre los dispositivos hardware, y, utilizándola, se decida el nodo concreto que va a ejecutar cada acción (enviando la orden correspondiente para su utilización, enviando el mensaje acorde con su sintaxis o modelo de datos). 
En esta sección se presenta de manera general la tecnología propuesta para gestionar la ejecución a bajo nivel de servicios provistos desde el entorno cloud o el dispositivo que denominamos mula.

Esta solución considera un proceso de recolección de datos que se emplea para determinar el coste de ejecución y la QoS ofrecida para cada dispositivo. Además, utilizando un procedimiento de selección probabilística propuesto en este trabajo, el dispositivo que va a estar encargado de realizar la ejecución es seleccionado de forma dinámica. Dependiendo de la implementación, los dispositivos seleccionados pueden tener la autoridad para delegar la ejecución (paradigma comúnmente descrito como coreografía) o necesitan de un controlador hardware para que coordine el proceso completo (orquestación).

El escenario propuesto para el modelo de ejecución se describe en la Figura 22. Cada red de sensores actúa como una red independiente, aunque se encuentra conectada con otrAs a través de la cloud (que representamos como de manera funcional como un gestor de servicios) y los dispositivos móviles DTN. Estos componentes mantienen un listado de los servicios disponibles, que se ofrecen a los entornos WSAN para su ejecución delegada. El gestor de servicios debe (además de disponer de otras funcionalidades) determinar si servicios con distinto identificador realmente corresponden a un mismo servicio, y gestionar su ciclo de vida.

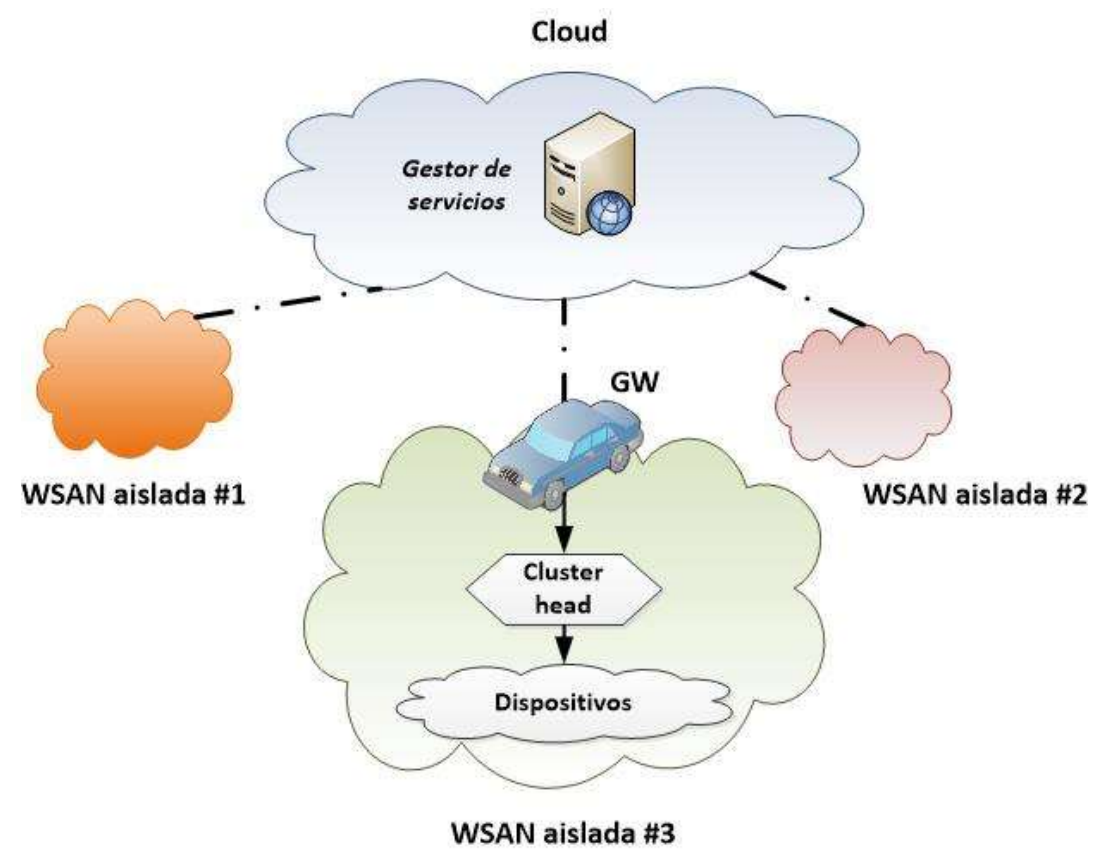

Figura 22: Arquitectura WSAN de ejecución

En este contexto, una solución para la gestión de servicios debe considerar tres niveles (Figura 23):

1. El nivel de conexión representa todos los procedimientos requeridos para integrar los sensores y actuadores periféricos con la unidad de micro-control central de cada dispositivo (conexión física) (Bordel, Rivera, \& Alcarria, 2016). 
Este nivel también presenta la solución para la conexión de cada cluster head con otros cluster head de la WSAN y los dispsoitivos móviles DTN (interoperabilidad). Los mecanismos de interoperabilidad deben ser implementados en los microcontroladores en los cluster head.

2. El nivel lógico incluye las tecnologías para la coordinación que necesita el dispositivo móvil DTN (mula) para ejecutar servicios de bajo nivel utilizando la WSAN subyacente. Algunas soluciones de este nivel lógico ya han sido descritas en este trabajo de tesis, como el proceso de matchmaking o la selección de dispositivos.

3. El nivel de servicios integra todas las tecnologías que convierten los servicios de bajo nivel en entidades más abstractas y más cercanas a la comprensión humana. Se consideran los subniveles de servicios DTN (que integra servicios con una correspondencia directa con el hardware sobre el que se va a ejecutar), servicios de acceso (representa servicios expresados en un lenguaje de ejecución) y aplicaciones prosumer (que integra servicios descritos en un lenguaje de descripción de servicios o SDL). Los diferentes niveles descritos, se corresponden con los niveles funcionales generales identificados en la Figura 7.

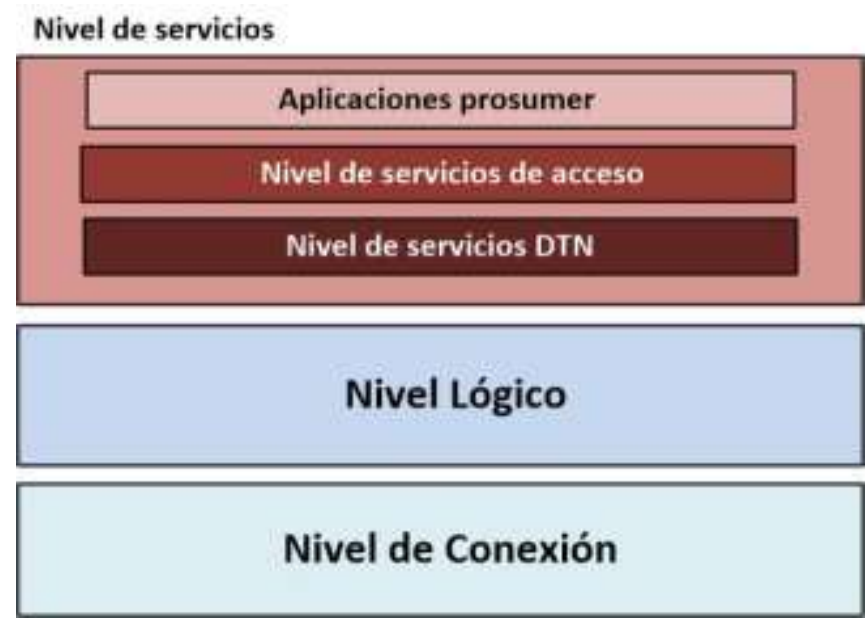

Figura 23: Gestión de servicios en redes DTN

En esta sección nos centramos en el nivel lógico, ya que contribuye a resolver las carencias de trabajos de investigación que se centren en tecnología de ejecución de servicios a nivel lógico.

En los siguientes aparatados se proponen:

1. Un mecanismo para la monitorización de la WSAN

2. Una técnica para la ejecución de servicios través de la gestión los cluster head de forma individual

3. Una mejora de la técnica anteriormente descrita para la gestión de cluster head a nivel grupal. 


\subsection{Monitorización de la WSAN}

La tecnología propuesta considera que los servicios no incluyen indicaciones sobre la calidad de servicio que debe soportar el nivel de comunicaciones. De todas formas, para optimizar el consumo de recursos escasos, la selección del dispositivo óptimo que puede soportar la ejecución de un servicio considera el estado actual de la WSAN. Un proceso de monitorización de dipositivos es necesario en este caso, incluyendo los siguientes tipos de información: el coste de ejecución $\mathrm{Q}$ y una colección de indicadores de calidad a bajo nivel, que llamaremos $\Sigma$, como, por ejemplo, la disponibilidad.

La Figura 24 presenta una arquitectura funcional del escenario de aplicación. La información acerca de la WSAN se proporciona a través de los cluster head (componentes que implementan funciones de interoperabilidad), por lo que los costes son distintos para cada servicio y localización (es decir, para cada cluster head).

Desde el punto de vista matemático, los costes forman un conjunto $Q$ de la siguiente forma:

$$
Q=\left\{q^{1}, q^{2}, \ldots, q^{N}\right\}=\left\{\left\{q_{1}^{1}, q_{2}^{1}, \ldots, q_{M_{1}}^{1}\right\}, \ldots,\left\{q_{1}^{N}, q_{2}^{N}, \ldots, q_{M_{N}}^{1}\right\}\right\}
$$

Donde $\mathrm{N}$ representa el número de cluster head y $M_{i}$ el número de servicios utilizando el cluster head $i$. Por tanto, $q_{j}^{i}$ es el coste de ejecución del servicio $j$ en la localización $i$.

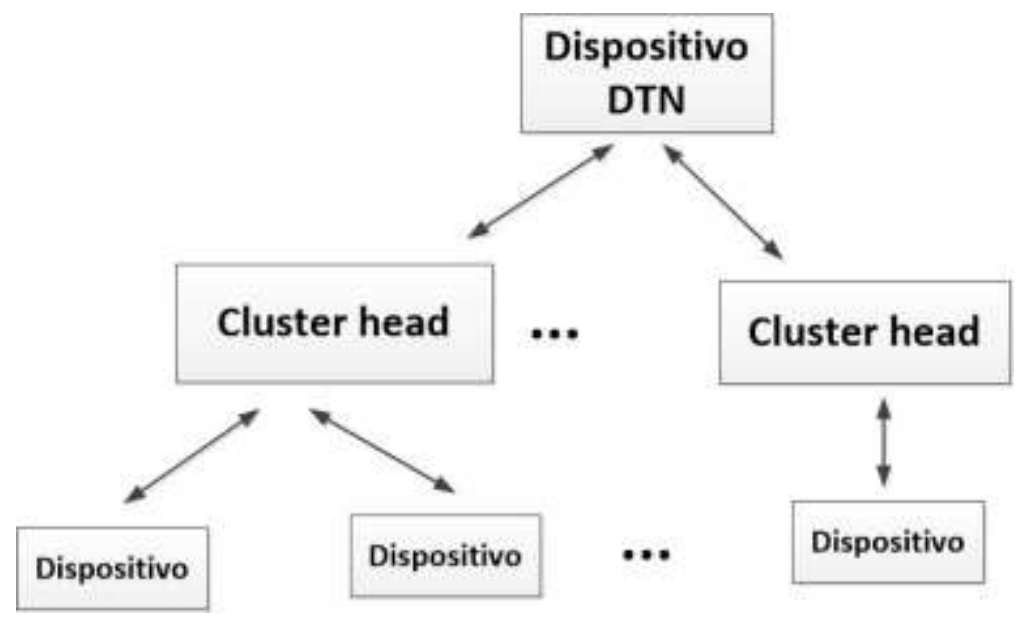

Figura 24: Arquitectura funcional del escenario de aplicación

Por otra parte, para cada localización y servicio, un conjunto de valores es utilizado como indicadores de calidad a bajo nivel (2). La siguiente lista representa los indicadores de calidad (3):

$$
\begin{gathered}
\Sigma_{j}^{i}=\left\{\sigma_{1}^{i, j}, \sigma_{2}^{i, j}, \ldots, \sigma_{R}^{i, j}\right\} \\
\Sigma=\left\{\Sigma^{1}, \Sigma^{2}, \ldots, \Sigma^{N}\right\}=\left\{\left\{\Sigma_{1}^{1}, \Sigma_{2}^{1}, \ldots, \Sigma_{M_{1}}^{1}\right\}, \ldots,\left\{\Sigma_{1}^{N}, \Sigma_{2}^{N}, \ldots, \Sigma_{M_{N}}^{1}\right\}\right\}
\end{gathered}
$$


Siendo $\mathrm{R}$ el número de indicadores considerados. Estos indicadores de calidad (como el tiempo de respuesta o la disponibilidad) pueden ser medidos directamente, y presentan expresiones conocidas (Shenker, Partridge, \& Guerin, 1997). Sin embargo, el coste de ejecución tiene que ser obtenido a través de un procedimiento no estándar (4)

$$
q_{j}^{i}=f_{\text {coste }}\left(q_{j_{\text {admin }}}^{i}, q_{j_{\text {hardware }}}^{i}\right)
$$

El coste del servicio j en la localización i se obtiene a través de una función de coste que considera dos contribuciones: un coste especificado por el administrador $q_{j}^{i}$ user (empleado para penalizar cierto equipamiento hardware, relación de balanceo de carga, etc.) y un coste hardware representando el consumo de recursos $q_{j}^{i}$ hardware Dependiendo de si ambas contribuciones son independientes o de si pueden compensarse entre ellas, la función de coste de ser una media aritmética ponderada (5) o una media geométrica ponderada (6) de estas dos variables:

$$
\begin{aligned}
& f_{\text {coste }}(\cdot ;)=\lambda_{1} q_{j_{\text {admin }}}^{i}+\lambda_{2} q_{j_{\text {hardware }}^{i}} \\
& f_{\text {coste }}(\cdot ;)=\sqrt{q_{j_{\text {admin }}}^{i} \cdot q_{j_{\text {hardware }}^{i}}^{i}}
\end{aligned}
$$

Finalmente, el coste hardware se obtiene como una media ponderada de una colección de indicadores de consumo de recursos $\psi_{k}$, como pueden ser el consumo de batería o el tiempo de procesameinto requerido por el dispositivo. Estos indicadores tiene que ser definidos para tomar valores en el intervalo [0,1], siendo 1 el valor que indica un coste mayor.

$$
q_{j_{\text {hardware }}}^{i}=\sum_{k} \beta_{k} \cdot \psi_{k}
$$

En la sección 8.4 consideramos que el dispositvo DTN (mula) pregunta a un solo controlador hardware si es capaz de ejecutar un servicio a bajo nivel (ejecución de servicios a través de gestión individual de cluster head). En la sección 8.5 el dispositivo móvil DTN realizar la pregunta a varios cluster head (ejecución de servicios a través de gestión grupal).

\subsection{Ejecución de servicios a través de gestión individual de cluster head}

Los servicios de bajo nivel pueden clasificarse en varios tipos dependiendo de los requisitos geográficos (ver Figura 25): 


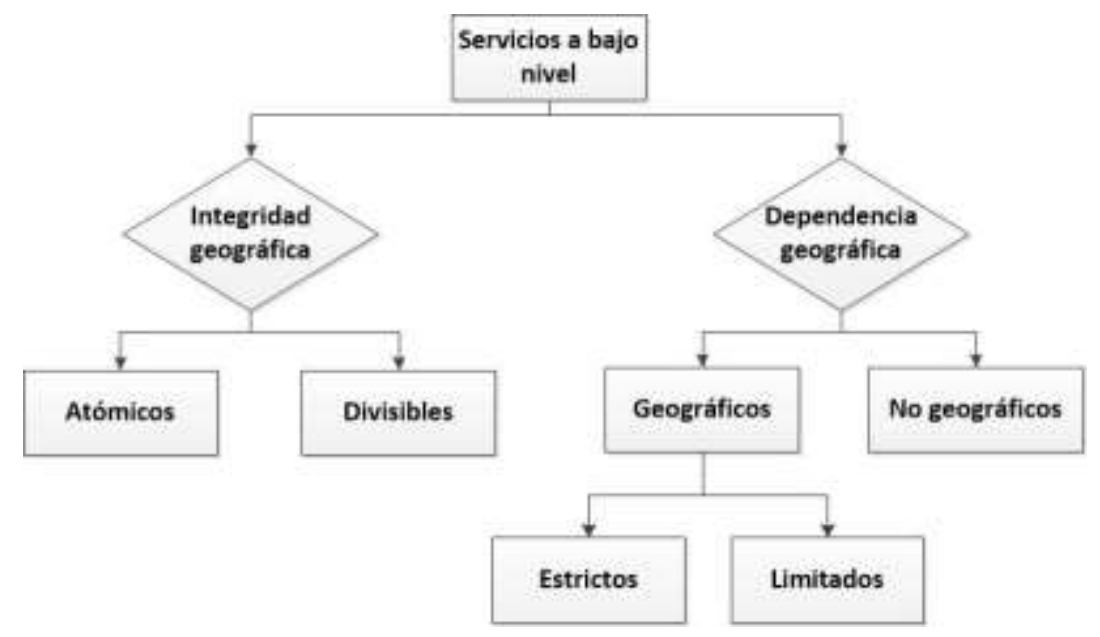

Figura 25: Clasificación de servicios dependiendo de los requisitos geográficos

Los servicios atómicos son los que tienen que ejecutarse enteramente en la misma localización y a través del mismo cluster head. Los servicios que puedan descomponerse en fragmentos y que pueden ejecutarse en varios cluster head reciben el nombre de servicios divisibles. Por otra parte, los servicios geográficos deben ejecutarse en una localización particular (geográficamente estrictos) o en un conjunto de localizaciones pertenecientes a un grupo (geográficamente limitados). Los servicios geográficos tienen que ser complementados con metadatos que describan las limitaciones geográficas. Los servicios con independencia geográfica pueden ser ejecutados en cualquier localización y controlador hardware.

Cuando una invocación de servicios es recibida por el dispositivo móvil DTN, este determina el conjunto de localizaciones $\mathcal{L}$ en las que puede ser ejecutado el servicio (dependiendo de restricciones geográficas, de la capacidad de cada cluster head, y los parámetros obtenidos mediante la monitorización de la WSAN). Con la información disponible se construye una función de probabilidad, tras lo cual pueden seguirse diferentes estrategias para la ejecución del servicio. En esta sección consideramos que el gestor de hardware interactúa de forma individual con los controladores hardware.

Las Figura 26 a Figura 30, representan varios diagramas de secuencia describiendo el procedimiento completo. 


\section{Caso A: Ejecución exitosa en un solo}

\section{paso}

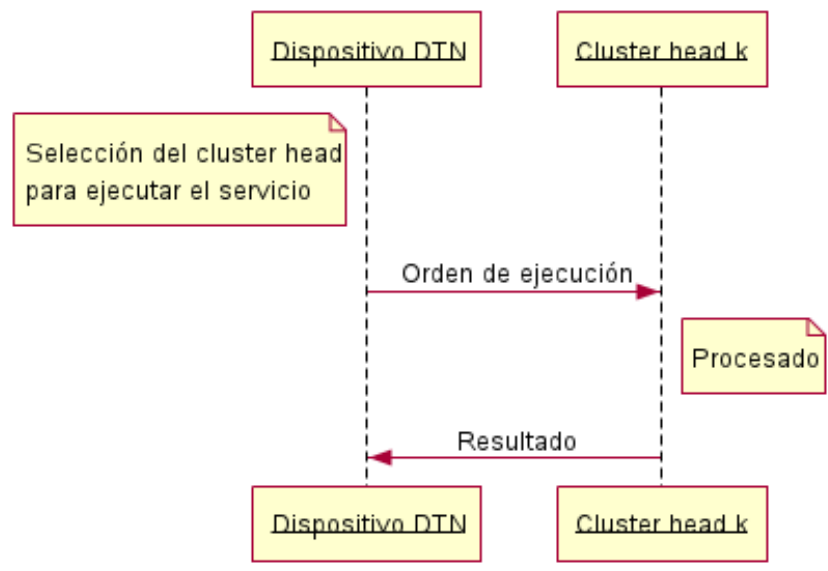

Figura 26: Diagramas de secuencia de ejecución para el caso A

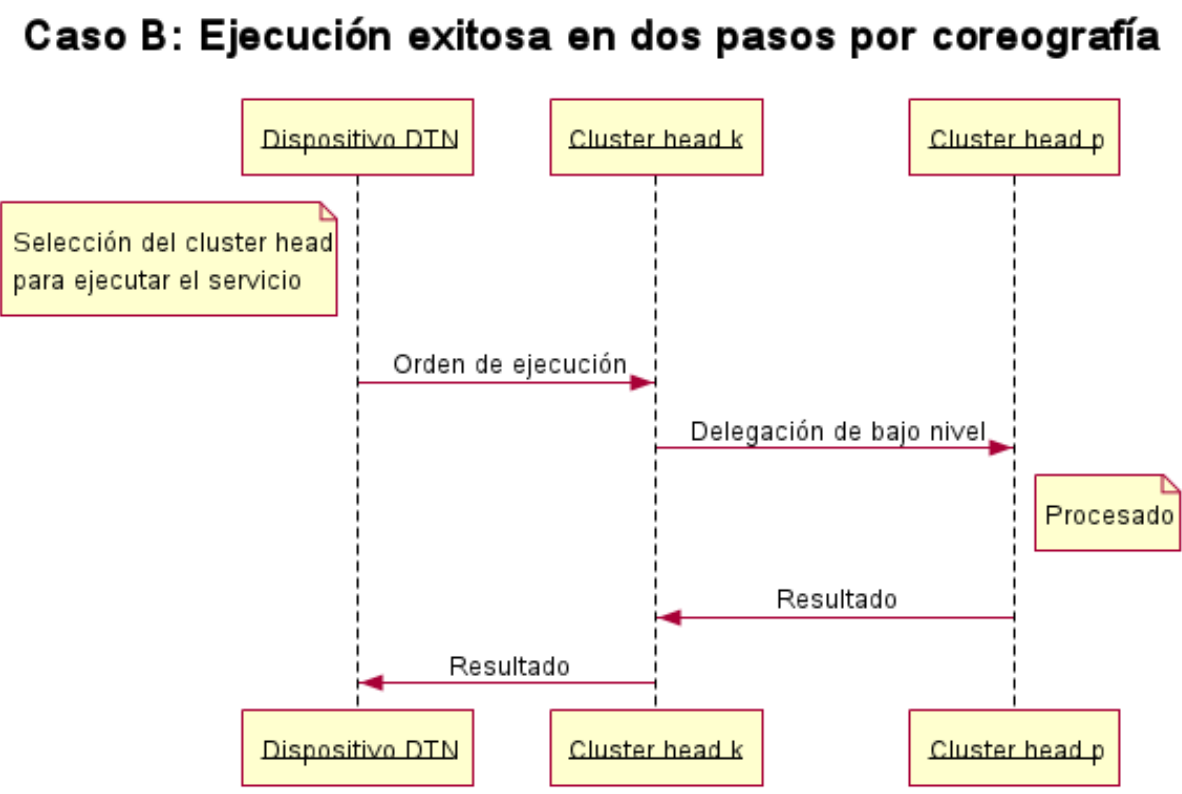

Figura 27: Diagramas de secuencia de ejecución para el caso B 


\section{Caso C: Ejecución exitosa en dos pasos por orquestación}

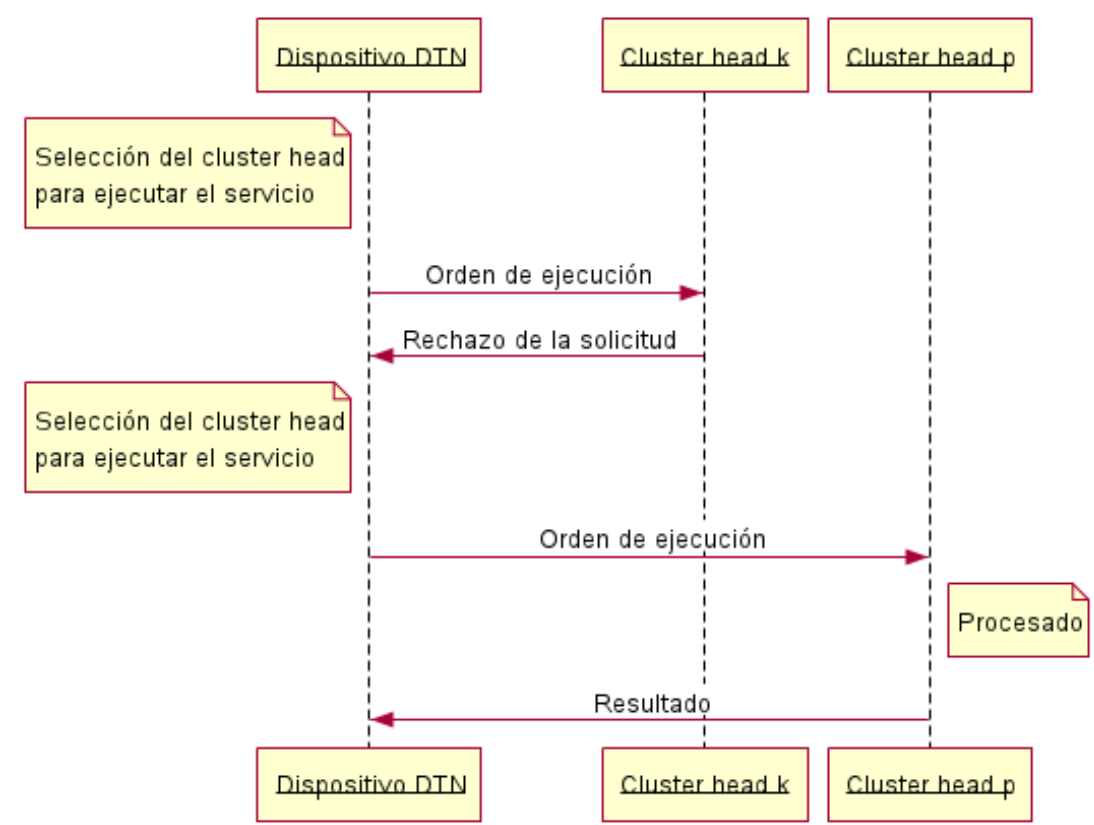

Figura 28: Diagramas de secuencia de ejecución para el caso C

\section{Caso D: Ejecución fallida por falta de respuesta}

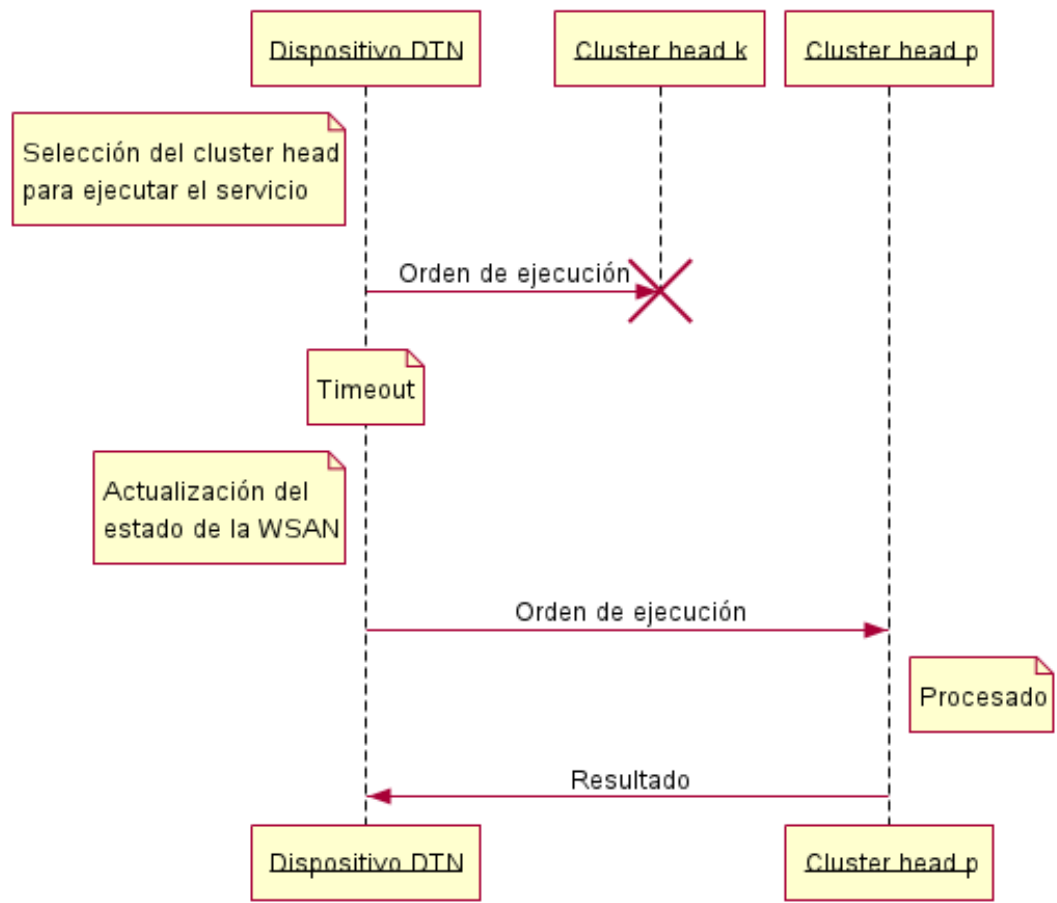

Figura 29: Diagramas de secuencia de ejecución para el caso D 


\section{Caso E: Ejecución fallida por retrasos en la red}

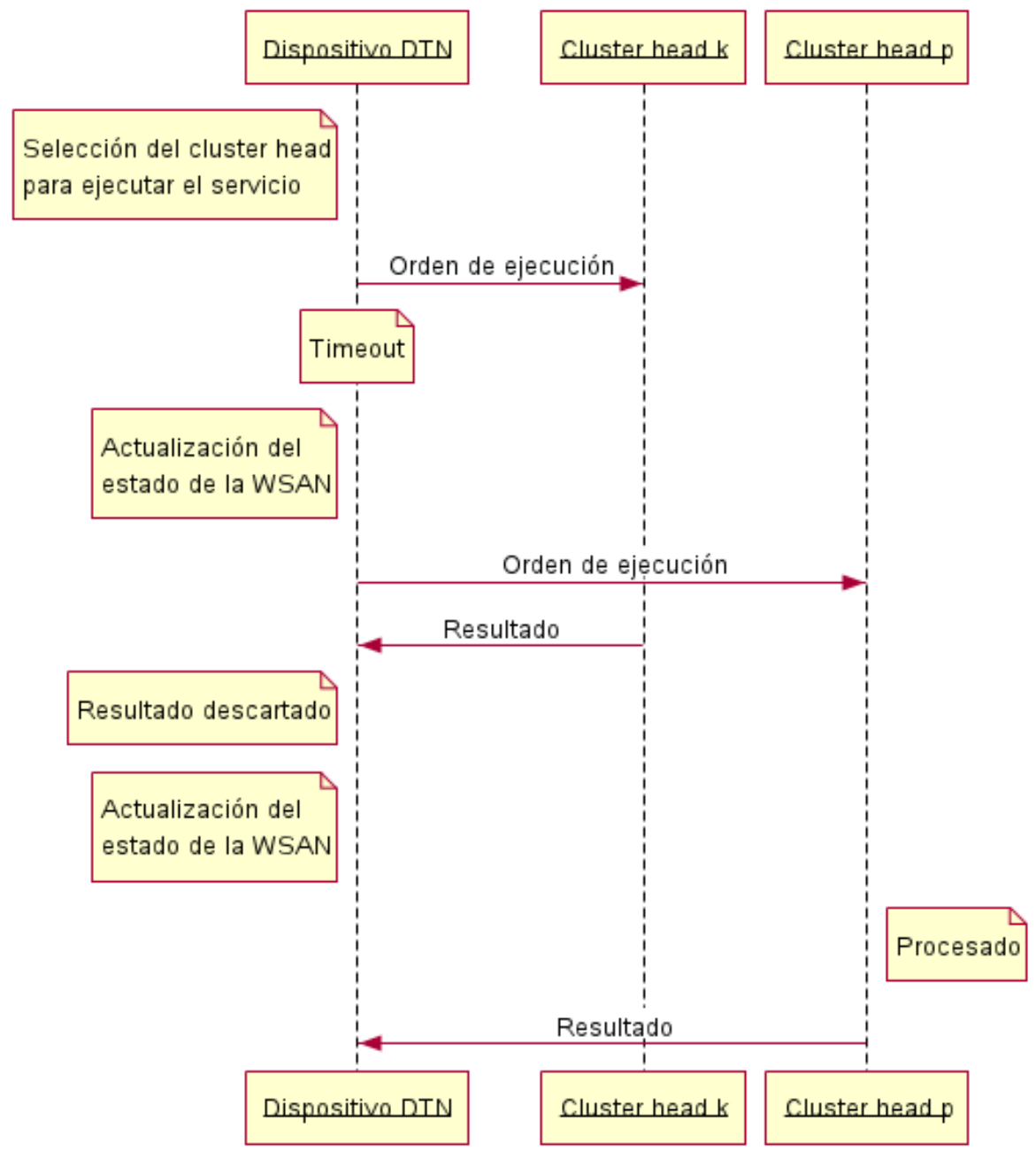

Figura 30: Diagramas de secuencia de ejecución para el caso $\mathrm{E}$

Se documenta brevemente cada paso:

1. El dispositivo DTN selecciona la localización (es decir, el cluster head) para la ejecución del servicio, siguiente la función de probabilidad expresada anteriormente.

2. El dispositivo DTN envía la orden de ejecución al cluster head seleccionado utilizando el formato de datos y la tecnología de comunicación adecuada. Típicamente se emplearán protocolos propietarios orientados a bytes (Bordel Sánchez, Alcarria, Martín, \& Robles, 2015) y comunicaciones de bajo consumo (como las que siguen el estándar IEEE 802.15.4 (Callaway et al., 2002)).

3. Si el cluster head seleccionado puede ejecutar el servicio, la ejecución queda realizada en un solo paso (Caso A, ver Figura 26). El cluster head realiza las acciones adecuadas y devuelve el resultado al dispositivo DTN. 
4. Si la orden de ejecución no puede ser satisfecha, pero el cluster head tiene recursos suficientes para gestionar la petición, hemos definido dos posibilidades.

a) Si el modelo de interoperabilidad seleccionado es de coreografía, el cluster head podría delegar la ejecución del servicio a otra localización (si está disponible). Este segundo cluster head ejecutará el servicio y enviará el resultado directamente al dispositivo DTN (Caso B).

b) Si el modelo de interoperabilidad seleccionado es de orquestación el cluster head (cluster head \#k en la Figura 28) debe enviar un mensaje de denegación al dispositivo DTN (caso C). En este momento, además, el dispositivo DTN activa un temporizador. Si cuando finaliza el temporizador no recibe respuesta (caso D), el estado de la WSAN es actualizado (los servicios ofrecidos por ese cluster head se declaran como no disponibles, el temporizador se amplia, etc.); y se realiza una nueva selección (eliminando la localización no disponible).

5. Finalmente, en ciertas ocasiones, el cluster head puede enviar un resultado al dispositivo DTN después de un tiempo de espera (caso E). En el momento de su recepción, el resultado se descarta ya que una nueva orden de ejecución fue enviada. Sin embargo, en este caso el estado de la WSAN cambia a disponible (especificando que los servicios de este clutser head están disponibles pero que el tiempo de respuesta es mayor de lo previsto).

Como principal ventaja de esta solución, el esfuerzo de ejecución se distribuye entre todos los cluster head disponibles, y el tiempo de ejecución de un servicio de bajo nivel puede verse reducido al mínimo (para una ejecución exitosa en un único paso). Sin embargo, este tiempo de ejecución es muy variable y puede aumentar considerablemente si la misma ejecución se produce múltiples veces hasta que tiene éxito. La Figura 31, representa de forma gráfica el tiempo de ejecuación descrito, para diferentes valores de la probabilidad de rechazo en un cluster head y diferente número de pasos necesarios para ejecutar un servicio. Como puede observarse, el tiempo de ejecución puede incrementarse hasta en dos órdenes de magnitud. Estos tiempos de variación en la ejecución de servicios pueden no ser tolerables para algunas aplicaciones, que necesiten una solución más estable. Este problema se aborda en el siguiente apartado. 


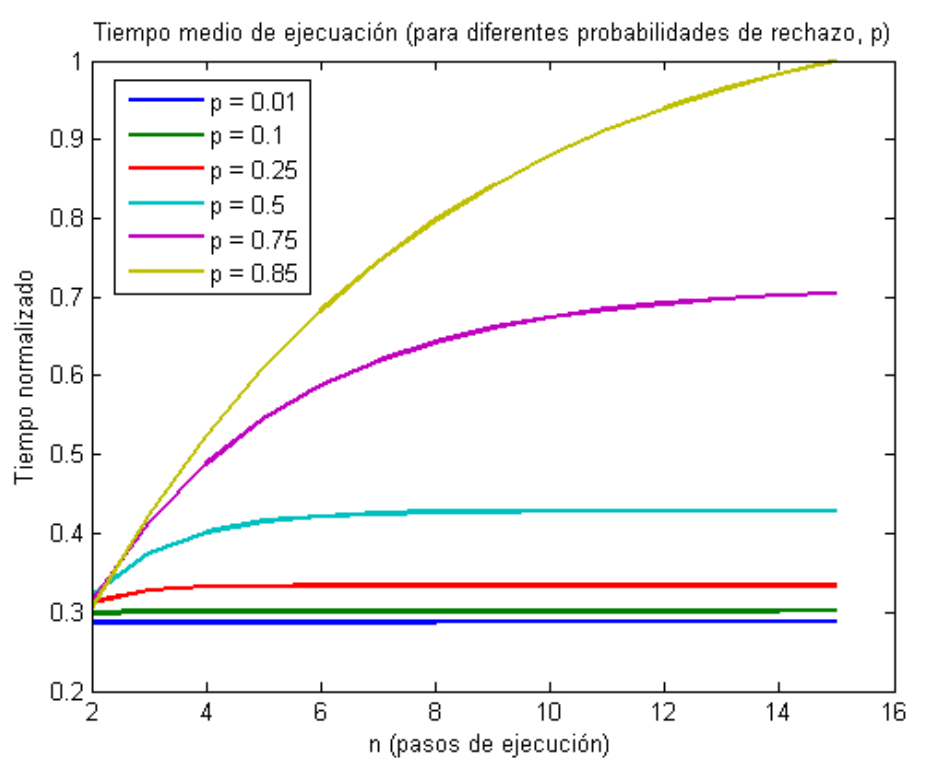

Figura 31. Representación gráfica del tiempo de ejecución en gestión de cluster head individual

\subsection{Ejecución de servicios a través de gestión de grupos}

Como se ha descrito anteriormente, en este apartado se incorpora una nueva fase en la que el dispositivo DTN pregunta a varios cluster head si pueden ejecutar un servicio específico.

Cuando una invocación de servicio llega al dispositivo DTN, éste selecciona un grupo de cluster head $U$, que puede ejecutar este servicio (siguiendo la función de probabilidad descrita en la sección anterior). Si el conjunto total de cluster head que son capaces de ejecutar el servicio es $V$, entonces tenemos un total de $\left(\begin{array}{l}U \\ V\end{array}\right)$ grupos. Entonces (ver Figura 32), una petición de ejecución es enviada a estos cluster head. El dispositivo DTN espera a las respuestas (se define un temporizador para evitar bloqueos en las comunicaciones) que pueden aceptar la petición (ofreciendo información adicional como el tiempo de procesamiento) o rechazarla. Dependiendo de las respuestas, se actualiza el estado de la WSAN.

Finalmente, considerando los cluster head que aceptan la petición de ejecución, se desarrolla en el dispositivo DTN una nueva función de densidad de probabilidad para determinar el dispositivo que debe ejecutar el servicio. En este punto, el procedimiento continúa de la forma descrita en el apartado anterior.

Esta fase inicial adicional provoca que el tiempo de ejecución sea mayor, pero presenta mayor estabilidad, como puede verse en la Figura 33, que representa de forma gráfica la evolución del tiempo de ejecución para distintos valores la probabilidad de rechazo en cada cluster head y para diferentes números de pasos de ejecución. Como puede verse, en este caso, aunque los tiempos son relativamente mayores, existe sólo 
una variación del 50\% entre el caso mejor y el peor (en el anterior procedimiento la diferencia podía estar en un $9900 \%$ ).

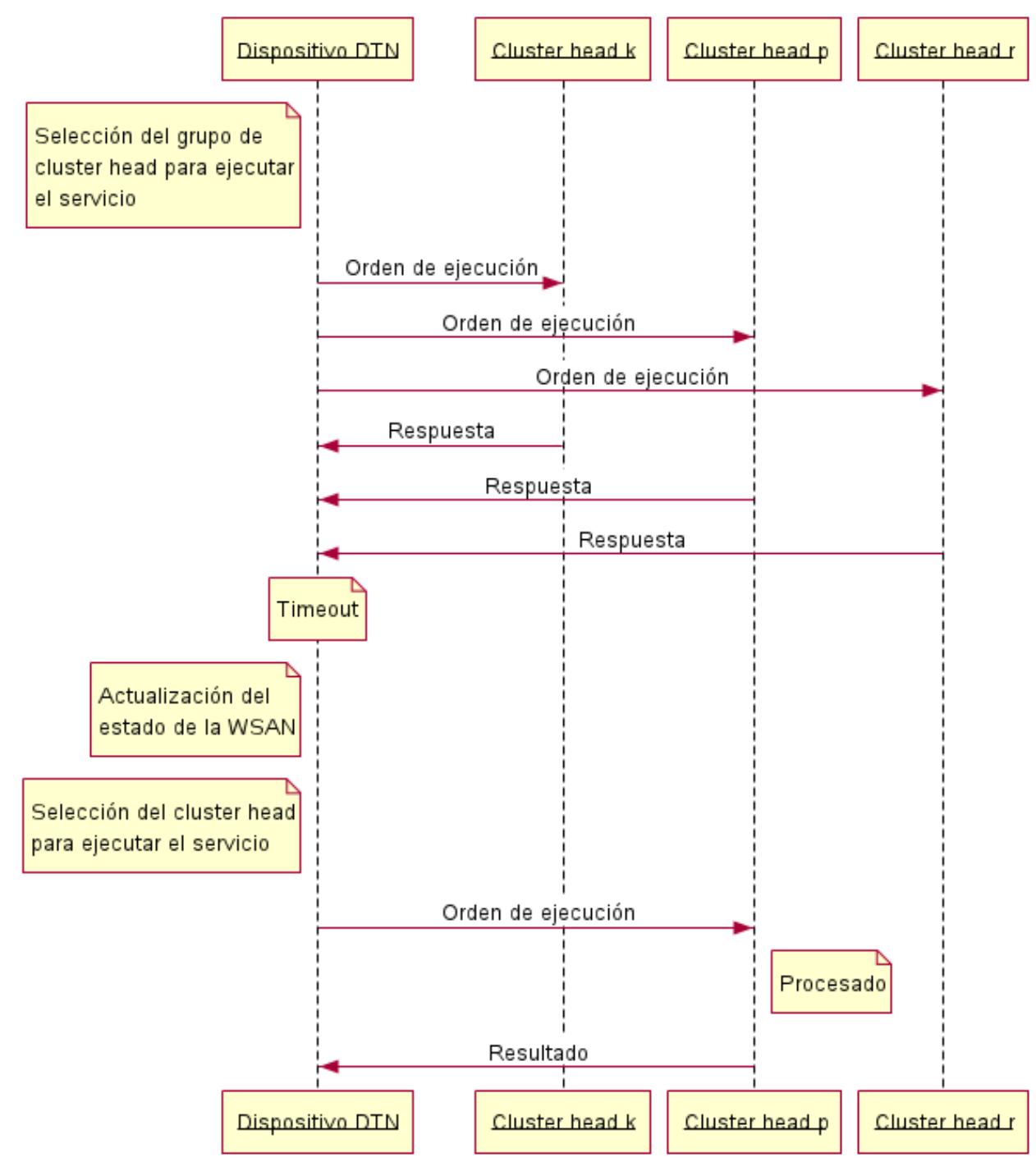

Figura 32: Diagrama de secuencia de una ejecución de servicios a través de gestión de grupos 


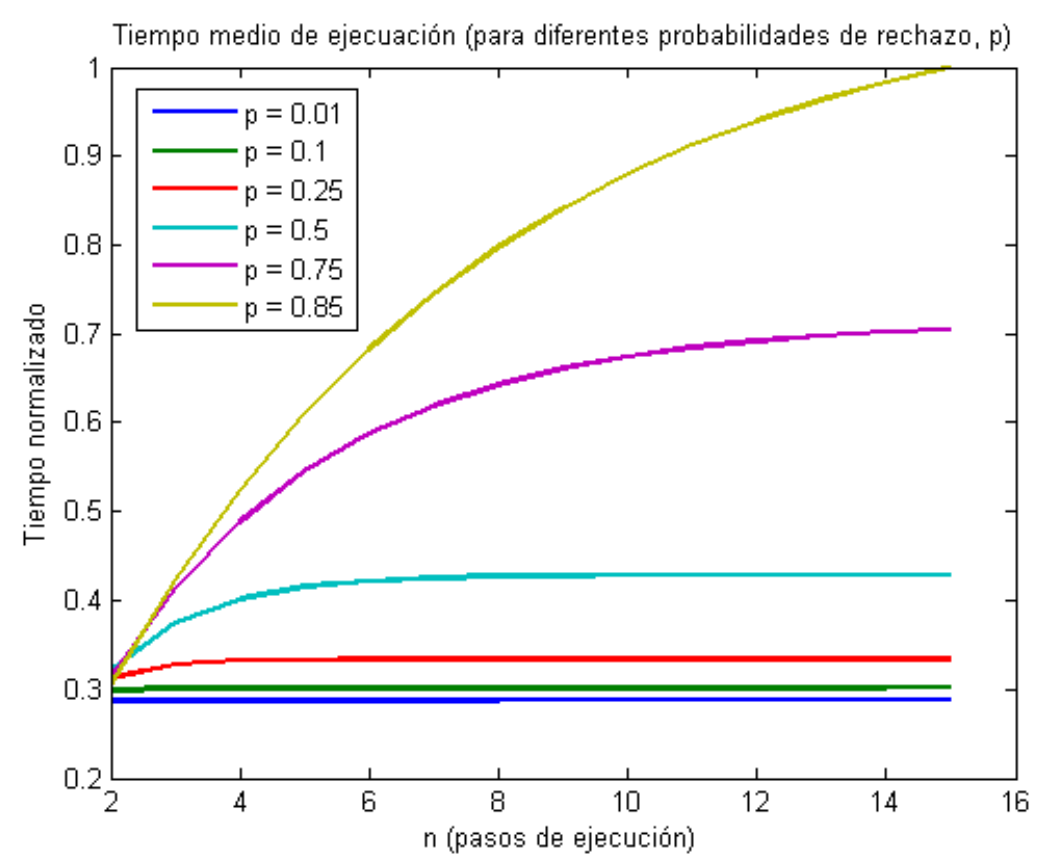

Figura 33. Representación gráfica del tiempo de ejecución en gestión de cluster head grupal

Considerando cualquiera de los procedimientos de gestión descritos en este trabajo de tesis, si no hay un cluster head que desee realizar la ejecución del servicio existen dos alternativas:

1. Si el servicio es atómico, la invocación es cancelada y se devuelve un mensaje de error al nivel superior.

2. Si el servicio es divisible, el dispositivo DTN puede descomponer el servicio y repetir los procedimientos con cada una de las partes. De la misma forma, si alguna de las partes no puede ser ejecutada, la invocación es cancelada en su conjunto y se devuelve un erro a los niveles superiores.

\subsection{Conclusiones}

Los entornos WSAN con características disruptivas requieren una solución para la gestión de servicios a bajo nivel. Esta solución debe abordar los procedimientos requeridos para asociar la ejecución de un servicio determinado con un dispositivo hardware.

En esta sección he propuesto una tecnología para la gestión de servicios a bajo nivel basado en tres procedimientos. Esta solución pertenece al nivel lógico de la arquitectura general del sistema.

El primer procedimiento considera un proceso de monitorización de WSAN para la evaluación del estado actual de la plataforma. El segundo describe un método para la ejecución y gestión de servicios basados en gestión individual de cluster head. El 
último procedimiento descrito añade un nivel más de complejidad, donde se propone una solución para la ejecución de servicios basados en gestión de grupos.

La validación experimental de estas soluciones se muestra en la próxima sección, donde se comprobará como las previsiones de retardo y rendimiento de cada solución a nivel teórico se cumplen a nivel de simulación. 


\section{Validación de escenarios, simulación y resultados}

\subsection{Introducción}

En este Capítulo se describe el desarrollo de una plataforma de simulación monolítica, liviana, configurable, flexible, altamente escalable y confiable, que proporcione soporte para simulaciones de ambientes distribuidos con comunicaciones limitadas, inalámbricas, disruptivas, y que permita la experimentación de las aplicaciones, prototipos y pruebas de conceptos, que validan las contribuciones en los distintos escenarios (Objetivo \#9).

En esta tesis se propone una solución para la ejecución de distribuida de servicios sobre las WSAN remotas. Esta alternativa está basada en la integración entre la capa de servicios, la capa de overlay network DTN, y los dispositivos en las WSAN. La solución que se presenta considera el modelo de servicio propuesto, la delegación, la ejecución remota de servicios móviles distribuidos, y los flujos de mensajes de gestión y de datos, requeridos por el sistema de administración de servicios en la cloud. Este sistema convergente permite, optimizar el rendimiento de la ejecución del servicio, mediante las estrategias de delegación y ejecución remota del mismo.

Los efectos de las características del perfil de los servicios distribuidos móviles requeridos para despliegue (ejemplo: centrado en el usuario o en la máquina), su naturaleza de ejecución (ejemplo: local o remoto), o las necesidades de red (Ejemplo: resistente a fallos y retardos), disponibilidad de los recursos (ejemplo: activos o inactivos - falta de energía), y geolocalización, se verán reflejados en el rendimiento de la arquitectura propuesta. Estos escenarios suponen un conjunto de desafíos para la ejecución de servicios distribuidos en entornos de WSAN remotas, como un nuevo modelo de servicio, mecanismos de distribución de contenidos que deben ser adaptados, mientras superan las limitaciones de los equipos que soportan roles activos en la ejecución de servicios en ambientes de movilidad. Algunas de estas limitaciones incluyen: heterogeneidad de los equipos, la necesidad de dinamismo y movilidad, 
recursos de hardware escasos, comunicaciones limitadas (tiempo, capacidad, errores), tolerancia a interrupciones y retardos prolongados.

En la siguiente sección se definen algunos escenarios en donde es aplicable la solución propuesta. De igual forma se describen los distintos casos de uso, que se derivan de las interacciones de los distintos participantes (roles), en la ejecución de servicios móviles distribuidos.

\subsection{Definición de escenarios de aplicación}

Los entornos que son considerados están enmarcados dentro del consenso del grupo de investigación Information-Centric Networking Research Group - ICNRG (Davies et al., 2015). Las ICN guardan una estrecha relación con las DTN, ya que utilizan una gran cantidad de las funcionalidades de esta última. En el RFC 7476, se aborda la problemática de la selección de los escenarios de validación en función de los diferentes enfoques de investigación, e intentan establecer un orden para poder comparar los diversos enfoques, mostrando sus ventajas y desventajas. Dicho consenso contempla situaciones, que incluyen el intercambio de contenido oportunista, el manejo de problemas de infraestructura durante situaciones de emergencia (Arumaithurai, Blefari-Melazzi, Tagami, Seedorf, \& Ramakrishnan, 2016) y la conectividad en áreas rurales remotas, sin provisión de Internet y poca o ninguna infraestructura de comunicaciones.

Los casos planteados en las secciones 6.5 y 7.4, están incluidos en el ámbito de la provisión de servicios de red, para el intercambio de contenidos oportunistas en áreas rurales remotas, donde no hay disponible infraestructura de comunicación, y tampoco acceso a Internet. En los siguientes apartados se presentan las abstracciones de los casos de uso de dichos escenarios, y luego se describen los requerimientos de los desafíos fundamentales en este contexto.

\subsubsection{Escenarios y Casos de Uso}

Los diferentes escenarios de aplicación considerados durante la investigación se describen en esta sección. El objetivo de estos escenarios y casos, es evaluar las distintas contribuciones propuestas en las secciones anteriores. La exposición y análisis se lleva a cabo de forma gradual en función de la complejidad de las temáticas consideradas en la investigación.

\section{Escenario monitoreo de granja agrícola remota}

El monitoreo de granjas agrícolas remotas, donde no existen comunicaciones de extremo a extremo, es una de las principales motivaciones de este trabajo de investigación. La provisión de servicios en este contexto, es un tema de investigación abierto, por las múltiples y complejas implicaciones (recursos limitados, movilidad, largos retardos y disrupciones en las comunicaciones) de las entidades participantes en estos escenarios. 
En el escenario de monitoreo de granja agrícola remota, existe la necesidad de adquisición de datos sobre la humedad en un sembradío de arroz. El objetivo del servicio de adquisición de datos es verificar continuamente las condiciones de humedad del suelo en el sembradío. La descripción de este escenario ha sido detallada en la sección 6.5.

La Figura 34, muestra la descripción gráfica de nuestro escenario elemental. Para este entorno se define el caso de uso general, en el cual se pueden observar los actores principales y las asociaciones entre ellos.

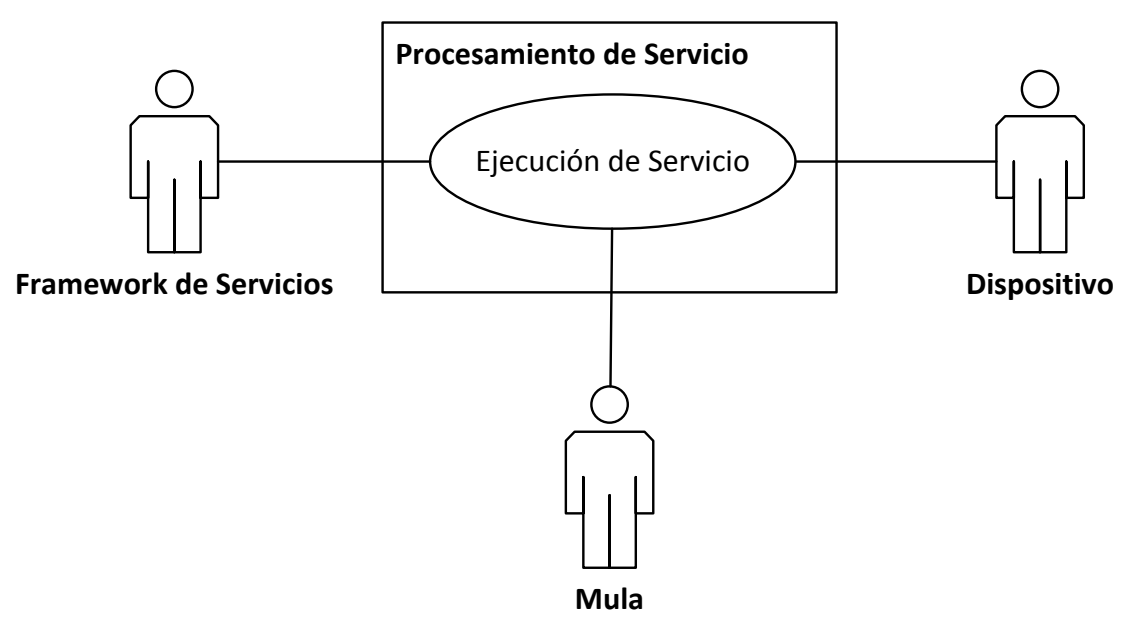

Figura 34: Caso de uso genérico

Este escenario cuenta con varios actores que participan en la ejecución del servicio, los cuales se describen con mayor detalle a continuación:

En primera instancia están los dispositivos, los cuales consisten en nodos coordinadores (cluster head) de las redes inalámbricas de sensores y actuadores, que se encuentran desplegadas en el sembradío. Los sensores se encargan de recolectar los datos y enviarlos a su nodo coordinador, el cual los almacena temporalmente, según sus características de almacenamiento. Este nodo cuenta con una interfaz de datos, a través de la cual puede aceptar peticiones de información, realizadas por otros nodos en su área de cobertura que cuenten con una interfaz de comunicación compatible.

La mula es un componente fundamental en la arquitectura. Esta entidad tiene características de movilidad, que le permite intercambiar mensajes DTN con los dispositivos (cluster head) dentro de su cobertura de radio, adicionalmente, puede comunicarse con el framework de desarrollo de servicios mediante una conexión a Internet.

Otro participante es el framework de desarrollo de servicios, que está accesible en la cloud. Esta herramienta permite la creación de servicios, mediante componentes. 
Los componentes se resuelven con las capacidades que la mula genera en función de los dispositivos que descubre.

El escenario de monitoreo de granja agrícola remota presenta dos casos de uso principales, los cuales se llevan a cabo en de forma asíncrona y espacialmente desacoplados. A continuación se analizan en detalle ambos casos, para mejor comprensión de las funcionalidades y las interacciones entre los participantes.

Primer caso de uso: Generación de capacidades

El primer caso es el resultado del contacto inicial entre una mula y un dispositivo, al cual denominamos generación de capacidades. La Figura 35, muestra el esquema gráfico de este caso de uso.

Los dispositivos pueden proporcionar dos tipos de recursos, los propios o locales (ejemplo: sistemas de posicionamiento global, sensor de contaminación ambiental por pesticidas, etc.), y los externos (remotos), que están accesibles mediante enlaces de comunicación (ejemplo: sensores de humedad en WSAN del sembradío). Cada dispositivo puede ofrecer sus recursos mediante una interfaz de datos ad-hoc. La heterogeneidad de los recursos que se pueden encontrar, se resuelve mediante las capacidades de las DTN, es decir, los cluster head cuentan con pequeños módulos de funcionalidades compatibles con las versiones estándares de DTN2 (ejemplo: $\mu$ DTNContiki e IBR-DTN), a través de la cuales se comunican con la mula de datos.

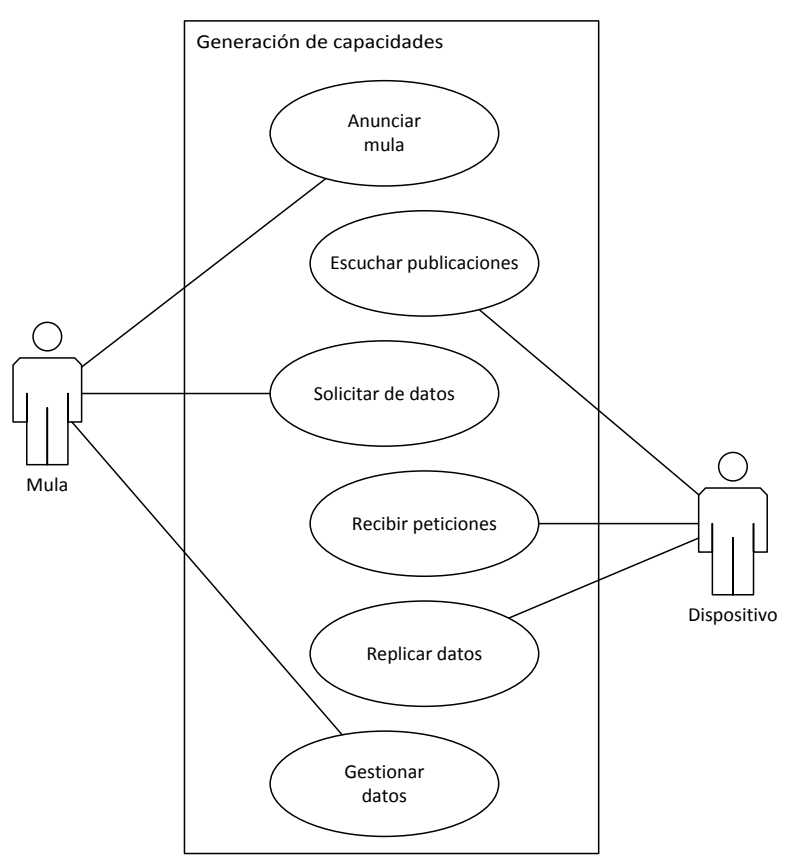

Figura 35: Caso de uso - Generación de capacidades

En el caso de uso generación de capacidades, la mula es una entidad que eventualmente puede tener la posibilidad de descubrir recursos, ofrecidos mediante los dispositivos. Para el proceso de descubrimiento la mula cuenta con varias interfaces de 
comunicación como Wi-Fi, Bluetooth, BLE, entre otras. Estas interfaces le permiten comunicarse con los cluster head de las WSAN. La mula es un participante móvil que puede tener unos patrones de movilidad que en principio son indefinidos. En el tiempo estos patrones pueden pasar a un estado con menor grado de incertidumbre, a lo cual llamamos conducta determinista. Las mulas móviles tienen la posibilidad de comunicarse con los dispositivos, con un paradigma oportunista. Estas entidades móviles descubren los dispositivos mediante anuncios periódicos de beacom. Los dispositivos están en un estado de escucha pasiva, hasta que se perciben un anuncio de mula o beacom. En este caso, los dispositivos responden al anuncio con otro beacom, mediante el cual se identifican y negocian el establecimiento de un canal de datos para el intercambio de bundles a nivel DTN. Cuando el canal de datos queda establecido, la mula puede generar peticiones de información sobre los recursos disponibles en el dispositivo. El cluster head, cuenta con un demonio a la escucha de peticiones de datos sobre la overlay network DTN. En el caso de que el dispositivo reciba una petición, este responderá con los datos solicitados, enviado un bundle de datos hacia las capas inferiores del stack para su transmisión. Este proceso de intercambio de mensajes se produce mientras la mula sigue en movimiento, con lo cual es de vital importancia optimizar al máximo la ventana de tiempo disponible para el intercambio de datos entre la mula y el dispositivo.

En la medida en que la mula recibe la información de los recursos que tiene disponible un dispositivo concreto, los procesa y almacena en forma de capacidades. La mula cuenta con un repositorio de capacidades que se actualiza cuando conoce un nuevo dispositivo o cuando hace contacto con dispositivos de forma recurrente, en cuyo caso renueva el estado de dichos recursos en repositorio de dispositivos a bordo de la mula. Las capacidades son generadas por la ejecución del algoritmo de descubrimiento, descrito anteriormente en la sección 6.3. El objetivo principal del algoritmo es el descubrimiento continuo de recursos, en función de los patrones de movilidad de la mula, y de los equipos que se encuentren en el radio de comunicación a su paso. El proceso de generación de capacidades es persistente en el tiempo, y permite un desacoplamiento entre las capacidades y los recursos.

\section{Segundo caso de uso: Resolución de componentes}

El segundo caso, es el que describe el evento de comunica entre la mula y el framework de servicios en la cloud. En la Figura 36, se puede apreciar la especificación gráfica del caso de uso resolución de componentes. 


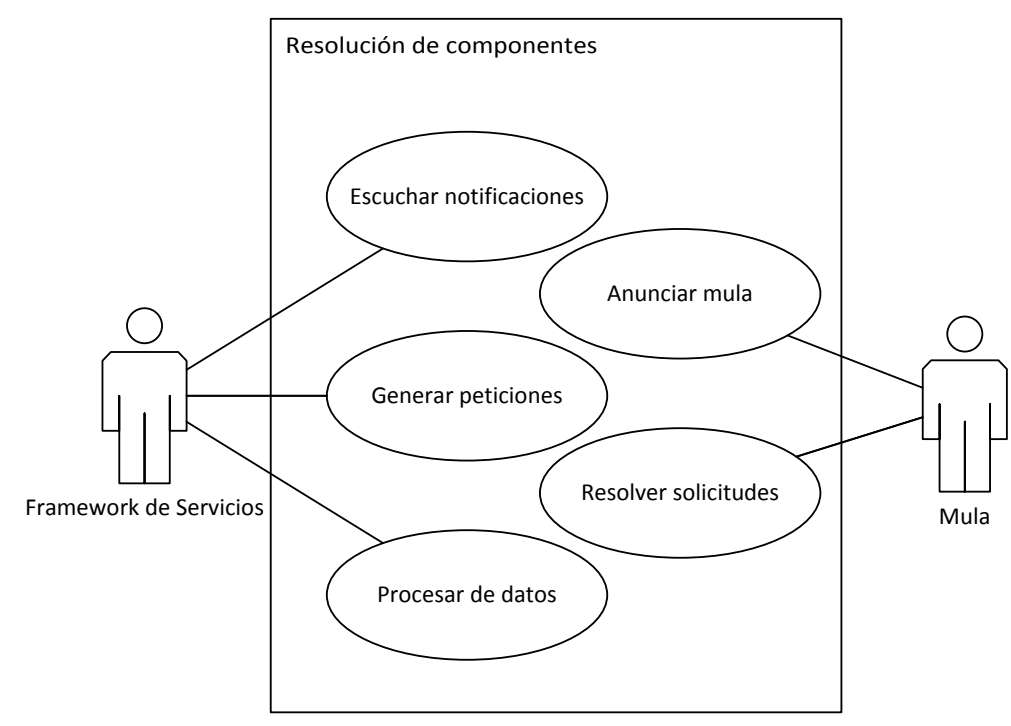

Figura 36: Caso de uso - Resolución de componentes

Los componentes de los servicios requieren de funcionalidades que pueden ser resueltas por las capacidades que están en los repositorios de las mulas. Una aplicación del framework de servicios escucha en un puerto específico las notificaciones, mediante el API DTN. Cuando arriba una notificación de mula, la capa DTN la recibe de las capas inferiores, la procesa, y luego la puede entregar hacia arriba, a la aplicación del framework que escucha estos eventos. Las mulas pueden notificarse ante el framework de servicios, cuando tengan disponible una conexión de banda ancha con acceso a Internet (GSM, WiMax, Wi-Fi u otros). Una notificación de mula provoca que la capa DTN procese la información de conexión, para establecer una canal de intercambio de datos mediante bundles DTN. Una vez establecido un canal de flujo de datos, la plataforma de servicios solicita información de capacidades a la mula. La mula recibe las peticiones e intentará resolverlas, y luego enviará una réplica con la información de capacidades que tiene en su repositorio a bordo, mediante el envío de bundles DTN. El framework de servicios utiliza la información de capacidades transmitida desde la mula, para procesarla mediante el algoritmo de MatchMaking $(M M)$, explicado en la sección 6.4.3. Este algoritmo gestiona la información de capacidades, con el objetivo de resolver los componentes con las capacidades disponibles después de la actualización de la última mula visible. El proceso de resolución dinámica de componentes se realiza de forma continua mediante el algoritmo de $M M$.

Este proceso se realiza bajo condiciones de movilidad de la mula, con lo cual la velocidad del proceso y las comunicaciones son vitales, para optimizar el tiempo de contacto. Es probable que este tiempo de visibilidad de la mula sea más prolongado, en comparación con los dispositivos, sin embargo, por los recursos con los que puede contar la mula es muy importante considerar que este tiempo de comunicación debe ser bien rentabilizado. 
El framework de servicios verifica en cada notificación de mula, si tienes servicios delegables. En tal caso, examina el RMD para comprobar su registro en este repositorio, de esta forma determina si está clasificada como determinista o indeterminista, y en función de esto realiza un tratamiento específico en cada caso mediante el algoritmo de delegación, el cual ha sido descrito en la sección 7.3. En la siguiente sección de aborda un caso de uso en donde se detalla la aplicación del mismo en una escenario concreto.

\section{Escenario de automatización de granja agrícola remota}

En el ámbito del control automatizado de la granja agrícola remota, existe la necesidad de comprobar continuamente las condiciones de humedad del suelo en un sembradío, para su posterior análisis y toma de decisión sobre la irrigación (volumen, tiempo, calidad, entre otros), con la finalidad de recuperar las condiciones adecuadas del suelo de la parcela, que permitan optimizar los recursos e incrementar la producción.

Considerando que la WSAN de la parcela no tiene comunicación directa con el sistema de regadío, existe la necesidad de procesar la información de humedad y comunicar al sistema de irrigación la información necesaria para el bobeo de agua hacia la parcela de arroz. Asumimos que la infraestructura de los ductos de agua hacia la parcela es funcional. La bomba que se encuentra a unos kilómetros tiene capacidades de comunicación con su cluster head. Este nodo puede comunicarse con los vehículos que tengan capacidades DTN, y que se muevan dentro de su rango de comunicación. El diagrama físico de este escenario se puede observar en la sección 7.4.

En este escenario interactúan varias entidades, como se puede apreciar en la Figura 37. Esta perspectiva muestra las interacciones entre los participantes y los roles dentro del escenario de estudio. Tomando en cuenta las características de movilidad de la mula, y el paradigma de comunicación oportunista, podemos decir que las interacciones generan múltiples casos de uso. La circunstancia que origina el primero caso, es el intercambio de mensajes en el que intervienen la mula, y la red de sensores de humedad de la parcela. El evento del contacto entre la mula y el sistema de irrigación, producen el segundo caso de uso. El tercer caso, es el producto de la comunicación entre el framework de servicios y la mula de datos. Estas situaciones, han sido consideradas en el primer escenario, sin embargo, hay tres casos presentes en este escenario, que no han sido analizados. A continuación se describen cada uno de los nuevos casos de uso en este escenario. 


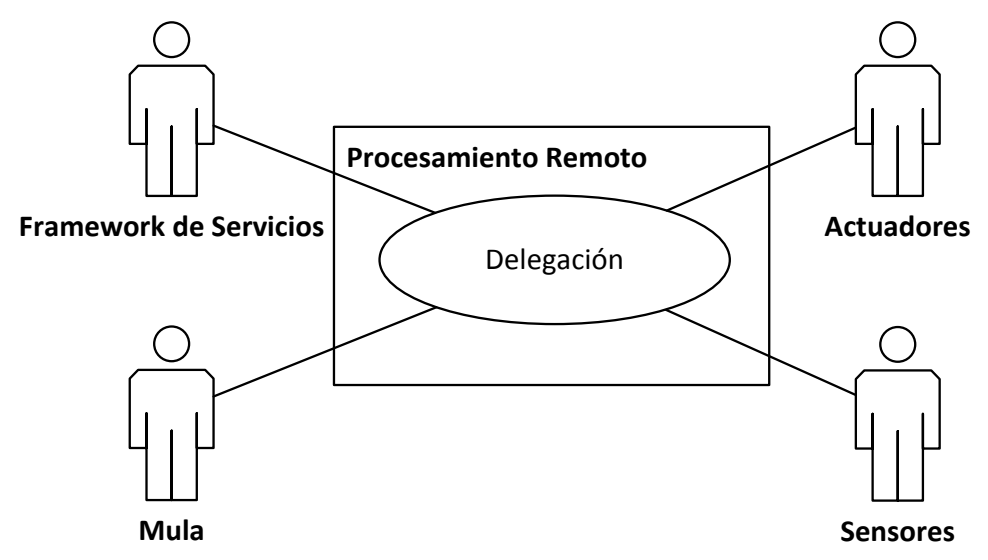

Figura 37: Delegación caso básico

Para el mejor análisis de este escenario de automatización de granja agrícola remota, hay que considerar algunas precondiciones establecidas por el propio escenario, las cuales han sido descritas detalladamente en el apartado 7.4. En este escenario asumimos una fase del caso de uso básico, en cual se originan los nuevos casos de uso que no han sido abordados en los escenarios anteriormente presentados. En este sentido, describimos las siguientes suposiciones:

- En algún momento previo, la mula de datos ha pasado cerca de la WSAN de la parcela e intercambió datos con el cluster head, generando las capacidades relacionadas con los recursos asociados a la red de sensores de humedad desplegados en la parcela. Esto ha provocado la realización del caso de uso "generación de capacidades", el cual fue explicado anteriormente.

- Asumimos el evento de intercambio de mensajes entre el cluster head asociado al actuador (bomba de agua) y la mula de datos. El contacto entre estas dos entidades, activa la realización del caso de uso "generación de capacidades", con la distinción de que las capacidades creadas están asociadas a recursos de tipo actuadores.

- Otra suposición importante, es que la mula se ha notificado con el framework, $\mathrm{y}$ ha intercambiado los bundles de datos necesarios para hacer de conocimiento del framework, todas las capacidades generadas mediante el descubrimiento de dispositivos. Este intercambio de mensajes es orientado por el caso de uso: "resolución de componentes", que ha sido explicado anteriormente. 


\section{Primer caso de uso: Desplazamiento de servicio}

La eventual notificación de las mulas, activa el procedimiento de delegación de servicios. Este procedimiento se realiza aplicando en algoritmo de "delegación de servicio" que se ha detallado en la sección 7.3.

El framework permite que los usuarios puedan crear servicios distribuidos, estos servicios pueden ser remitidos a una mula, para su posterior ejecución en forma remota. Un servicio puede ser delegado, solo bajo la especificación de las preferencias del usuario, considerando los requisitos del servicio y las capacidades de la mula. En la Figura 38, se muestra el caso de uso delegación de servicio, el cual ilustra gráficamente el contexto.

La delegación de servicio ocurre cuando se notifica una mula, y se valida sus recursos mediante peticiones de información sobre su disponibilidad de recursos, estos incluyen la información de su repositorio de capacidades $(\boldsymbol{M} . \boldsymbol{R} \boldsymbol{c})$. El procedimiento de delegación clasifica las mulas en deterministas e indeterministas, basado en esa clasificación realiza el procesamiento de delegación.

En la mula se ejecuta permanentemente un demonio del motor de ejecución de servicios a bordo. Este agente utiliza el API de DTN2 para escuchar peticiones de ejecución de servicios enviadas a la mula mediante los bundles de datos. A partir de esto, el motor de ejecución se encarga de gestionar la ejecución del servicio.

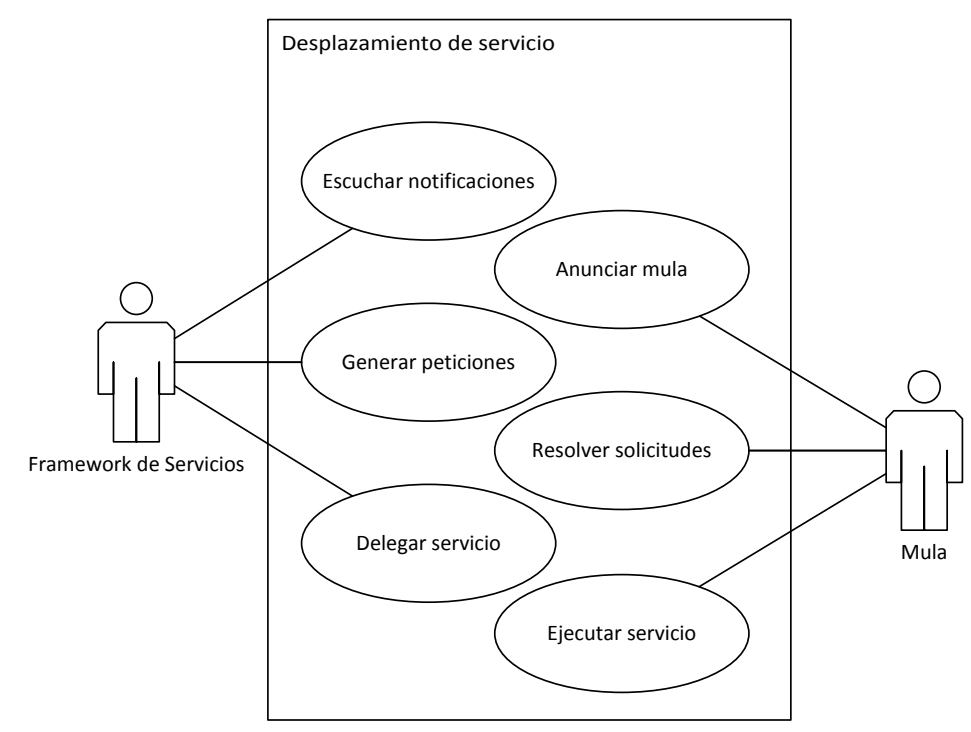

Figura 38: Caso de uso - Delegación de servicio

Una vez que el servicio de activación remota del regadío de la parcela se encuentra a bordo de la mula, este servicio intentará ejecutar las capacidades asociadas a los sensores y actuadores, relacionados con el servicio. El motor de ejecución de servicios solicita la ejecución de las capacidades enviando invocaciones de envío de bundles mediante el API de DTN2. En el caso de que la mula se acerque a la zona, y detecta 
que la posibilidad de comunicación con la parcela que geográficamente coincide con el servicio, procederá a enviar las peticiones de información (bundles de datos) al nodo cluster head. El nodo coordinador resuelve las solitudes de información mediante su interfaz de datos, y las envía mediante sus reducidas capacidades DTN. El motor de ejecución acepta los datos (desde las capas inferiores) los analiza mediante la lógica del servicio, y procede a invocar el siguiente componente del servicio a resolver. El servicio necesita invocar la capacidad asociada al actuador (la bomba) que se encuentra geográficamente distante de su ubicación actual. El siguiente caso de uso ilustra la resolución de dicha capacidad.

Segundo caso de uso: Ejecución de servicios de control

El estatus del caso anterior, y los atributos de movilidad de la mula, nos guía hacia el siguiente caso de uso. Este tiene lugar, en el momento en que el servicio delegado en la mula, requiere de la ejecución de capacidades asociadas otras WSAN que están distantes de la parcela del sembradío. Las capacidades necesarias para resolver el siguiente componente del servicio, está asociado al sistema de irrigación del escenario en estudio, que ha sido descrito anteriormente en la sección 7.4. La Figura 39, se puede observar el caso de uso, y los roles de las entidades necesarias.

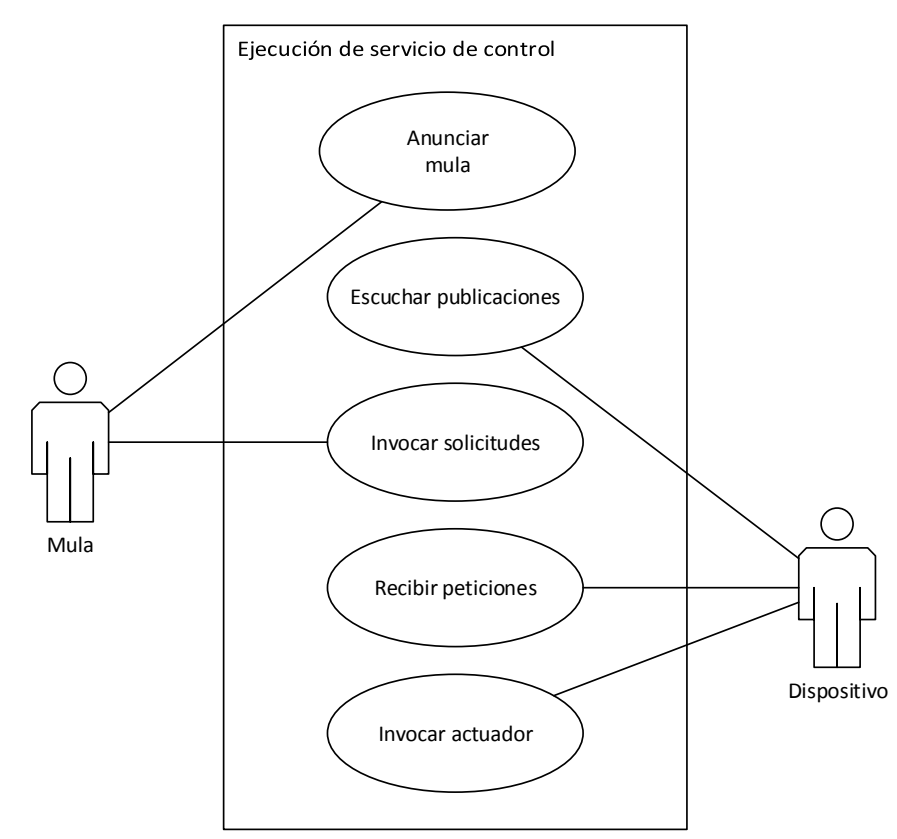

Figura 39: Caso de uso: Ejecución de servicios de control

En el ocasional encuentro con el dispositivo que gobierne a una WSAN, que permita ejecutar la capacidad necesaria, se darían lugar los siguientes eventos. La mula móvil se anuncia persistentemente en el tiempo. El dispositivo cluster head escuchas los anuncios de la mula, y cuenta con las capacidades (DTN) para establecer la comunicación con esta. El motor de ejecución de servicios a bordo de la mula, a través de su agente de escucha en la red DTN, determina que existe una posibilidad de 
invocación a un dispositivo que cumple con los requerimientos de la capacidad (geográficos, técnicos, restricciones, permiso, etc.), y está disponible para realizar la tarea de irrigación necesaria. En este caso, el motor de ejecución invoca la llamada a la ejecución de la capacidad, con todos los parámetros necesarios para su realización. Esta llamada es pasada al API DTN2, como una solicitud de envío de un bundle de datos hacia el cluster head que se ha identificado mediante un beacom.

Por otro lado en dispositivo, escucha la petición mediante sus capacidades DTN, y recoge los datos del bundle, mediante su interfaz de datos específica. Posteriormente, puede realizar la invocación al recurso del cual conoce todos sus datos a priori, ya que es parte de la WSAN que coordina. El actuador, escucha mediante su interfaz inalámbrica, la petición de operación enviada desde su cluster head, y la ejecuta según los parámetros de la invocación. Si la tarea del actuador se realizó correctamente, este envía un mensaje a su nodo coordinador, que intentará enviar los datos del resultado de la ejecución de la capacidad, hacia la mula que lo solicitó. La mula que solicitó la ejecución de la capacidad asociada a la irrigación de la parcela, puede conocer el resultado de la ejecución mediante el intercambio de mensajes con el nodo al que se lo solicitó, o a través de otros nodos que porten el mensaje de respuesta a esa petición. Otros nodos que se hayan acercado lo suficiente al nodo coordinador que necesita comunicar la respuesta a dicha petición, y que adicionalmente posean capacidades DTN, podrán ser portadores de mensajes de la red DTN (peticiones, respuestas, etc.).

En el framework, el servicio cambiará del estado "delegado" a "terminado", cuando los mensajes de la mula objeto de la delegación, arriben al framework de servicios informando, mediante un bundle de datos, que el servicio ha sido correctamente ejecutado. Estos mensajes pueden ser enviados desde la mula que ejecutó el servicio, o a través de otras entidades DTN que han estado en contacto con dicha mula.

Este caso de uso es análogo en el intercambio de mensajes, al caso de generación de capacidades que ha sido descrito en el anterior escenario de "monitoreo de granja agrícola remota". Sin embargo, un evento de interés que debemos analizar con detalle en este caso es la ejecución de los servicios en la mula o en el fremawork de servicios en la cloud. Anteriormente se ha mencionado, que el mecanismo de delegación permite alcanzar niveles altos de optimización en el tiempo de ejecución de los servicios, así como también una utilización eficiente del limitado tiempo de contacto con los cluster head en la WSAN.

\section{Tercer caso de uso: Ejecución de servicios a bajo nivel}

Una vez los servicios han sido delegados en los dispositivos móviles DTN más apropiados, es preciso que las mulas ordenen la ejecucion de los servicios a los dispositivos de las WSAN. En este contexto, se define este último caso uso.

Los dispositivos DTN deben buscar entre los cluster head con los que se pueden comunicar, aquellos capaces de acceder a las capacidades necesarias para ejecutar el 
servicio. En WSAN, múltiples dispositivos pueden ofrecer una misma capacidad, por lo que, en general, diversos cluster head pueden ofrecer las capacidades requiridas.

Dependiendo del servicio y sus requisitos diferentes soluciones han sido descritas en el Capítulo 8, cuyo escenario de aplicación es coincidente con este caso de uso.

\subsubsection{Identificación de requisitos}

En esta sección identificamos los requerimientos necesarios para una herramienta de simulación, para la ejecución de servicios distribuidos sobre WSAN. Como resultado del análisis previo, y las definiciones detalladas de los escenarios y casos de uso, identificamos un conjunto de requerimientos necesarios para la simulación de ejecución de servicios distribuido sobre WSAN remotas.

\section{Requisitos relacionados con la red:}

- Facilidad de configuración de interfaces de red inalámbricas compatibles con los estándares IEEE 802.11n.

- Desarrollo de distintas topologías inalámbricas, modo infraestructura o adhoc.

- Establecimiento de la cobertura de la señal inalámbrica, soporte de modelos de retardos de propagación y modelos de pérdida de propagación.

- Planificación de eventos de pérdida de los enlaces y restablecimiento de los mismos controlados en el tiempo.

- Configuración de TCP/IP v4.0 de forma manual y mediante DHCP.

- Debe brindar una alternativa para el análisis de los flujos de datos y de control, posterior a la simulación.

- Establecimiento de patrones de movilidad aleatorios o predefinidos mediante la lectura de datos de un archivo externo. Debe poderse controlar la velocidad de movimiento de los nodos.

\section{Requisitos relacionados con DTN:}

- Nodos que soporten la instalación de DTNv2.0 a nivel de aplicación, incluyendo los requisitos de esta.

- Archivo de configuración de parámetros DTN por medio de script de texto plano.

\section{Relacionados con los nodos:}

- Capacidad de almacenamiento de bundles de distintos tamaños.

- Soporte para instalación de módulo de software, a nivel de capa de aplicación.

- Configuración del stack TCP/IP. 
- Cada nodo debe ser independiente y realizar el procesamiento de todas las capas del stack.

- Capacidad de cambio en el protocolo de comunicación.

- Soporte de para dual-stack de protocolos.

\section{Relacionados con la herramienta:}

- Versatilidad de en la cantidad de nodos a desplegar.

- Facilidad en el arreglo o disposición de los nodos.

\subsection{Diseño y desarrollo de la plataforma de simulación}

Las características específicas de los escenarios propuestos, y los requisitos anteriormente puntualizados, requieren del desarrollo de una herramienta que permita recrear estos contextos lo más cercanos a los escenarios reales. Hasta donde nosotros conocemos no existe actualmente una herramienta que cubra estos requerimientos, por lo tanto, hemos desarrollado una herramienta de simulación basada en Linux y Ns-3.

Esta herramienta utiliza el concepto de paravirtualización, que permite que cada máquina virtual (nodo) se comporte como un computador independiente, mientras proporciona todas las facilidades de configuración de un ordenador real. El Ns-3, proporciona la capa de red de la herramienta. Este simulador de redes, posibilita recrear condiciones muy cercanas a las de las redes reales. Adicionalmente, ofrece facilidades para comunicarse con nodos reales, esto permite que los nodos en Ns-3 puedan intercambiar bidireccionalmente mensajes con el mundo real o físico.

\subsubsection{Arquitectura}

El esquema conceptual básico de la herramienta se puede observar en Figura 40. Este diseño arquitectónico nos permite desacoplar los planos de aplicación y el de red. 


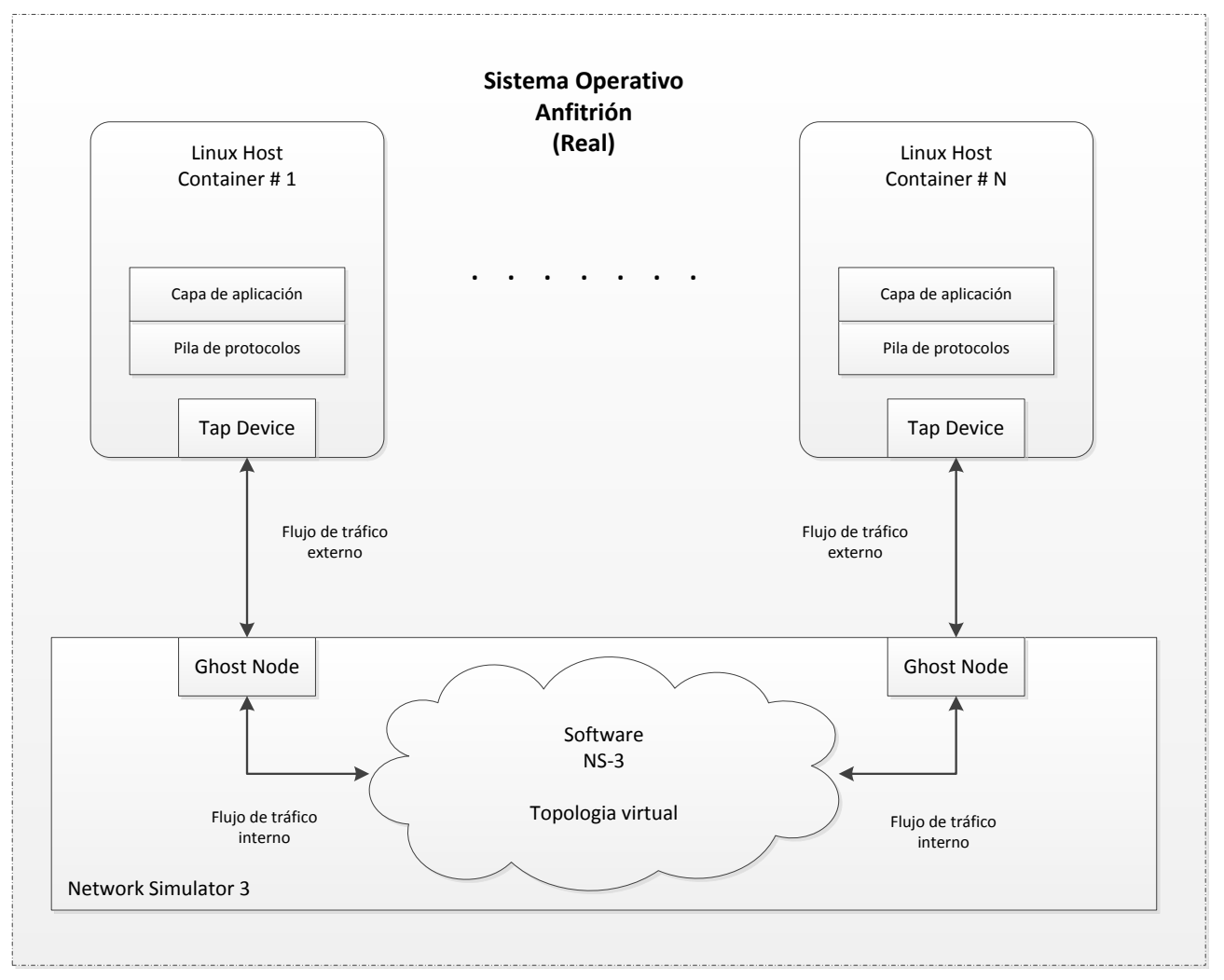

Figura 40: Arquitectura de herramienta de simulación

Una ventaja importante de la herramienta de simulación, es que soporta diversas topologías con un gran número de nodos, por lo tanto, los escenarios de alta densidad y escalabilidad están contemplados.

\subsubsection{Configuración de nodos}

Los nodos son creados sutilizando librerías LXC estándares de Linux. En la fase de configuración de los nodos algunos parámetros son definidos, como por ejemplo de dirección Ip, el modo de operación (ejemplo: físico o virtual), y puente. En general, para los escenarios planteados las interfaces de comunicación deben estar definidas como físicas ("phys"), ya que esta permite tener los nodos virtuales con la misma configuración que en un escenario real, pero, al mismo tiempo aislado a nivel del núcleo del sistema operativo.

Contamos la configuración del entorno de simulación para esos escenarios. Parámetros del ns-3, lo del Tap-Bridge, topología de red utilizada, lógica programada, etc.

\subsubsection{Abstracción de red}

Todos los parámetros de las topologías de red son provistos por un script que define los nodos, interfaces de red, conexiones y aplicaciones. Incluso cuando este enfoque supone mayor complejidad, este es más flexible que codificar toda la topología de la red. Es necesario definir dispositivos de tipo "TapBridge", para asegurar que Ns-3 y 
los contenedores se comuniquen de un lado a otro. En este caso, también establecemos las interfaces físicas correspondientes para los respectivos contenedores. El modo de operación del "TapBridge" es "ConfigureLocal". Este modo de operación permite crear automáticamente interfaces para que el "TapBridges" herede la misma configuración. Estos dispositivos se instalan en nodos Ns-3, como nodos fantasmas, para que ellos puedan comunicarse, a través de las mismas interfaces de red con Ns-3 o aplicaciones que puedan ejecutarse dentro del contenedor. Finalmente, en tiempo de ejecución, el Ns-3 crea el “TapBridge” y los contenedores se conectan a ellos.

\subsubsection{Despliegue de la herramienta de simulación}

La interface gráfica desarrollada para la herramienta de simulación, facilita la tarea de configuración y despliegue de los escenarios.

En la Figura 41, podemos observar en aspecto sencillo e intuitivo que permite un fácil entendimiento del funcionamiento de la misma.

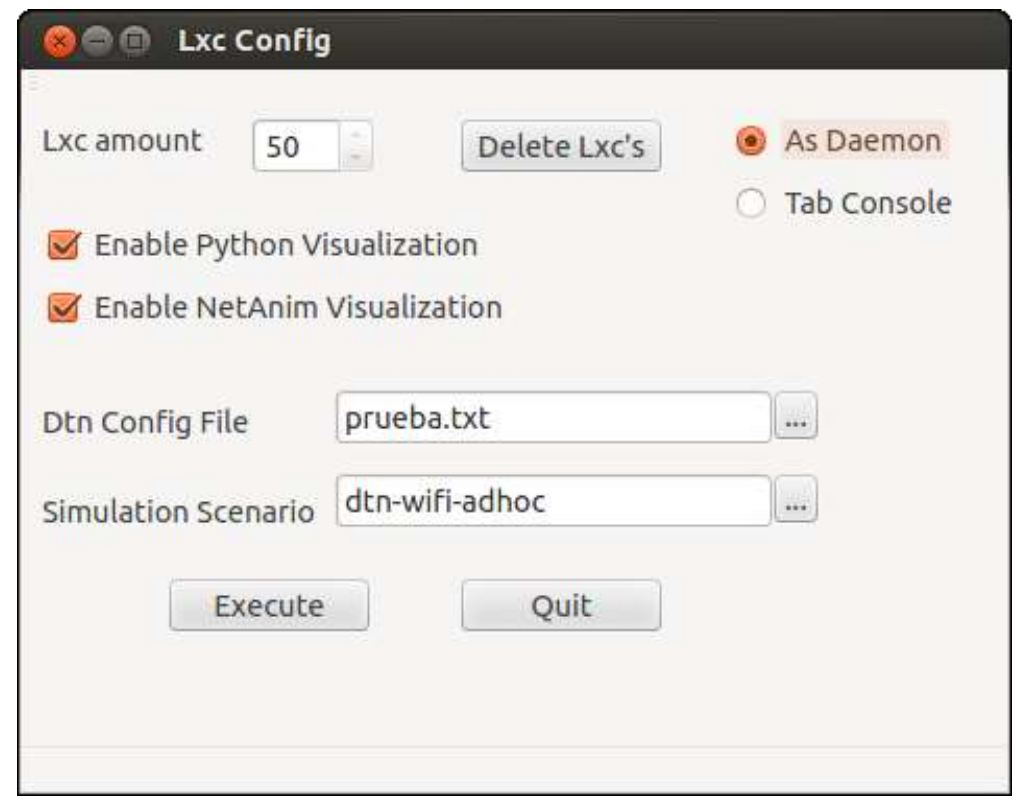

Figura 41: Interfaz gráfica de herramienta de simulación

Las principales funcionalidades que ofrece esta herramienta son las siguientes:

Especificación de la cantidad de nodos a utilizar. Este parámetros está limitado por el espacio de almacenamiento del equipo en se despliegue.

Existe un botón para borrar los nodos creados. Esto obedece a que el demonio DTN, tiene una máquina de estados persistentes, con lo cual se recomienda que en cada simulación, sean creados desde cero todos los nodos, garantizando un estado inicial en limpio.

Esta interfaz permite seleccionar el modo en que los nodos se muestran en el monitor, es decir, los nodos pueden ser creados en modos demonio, en cuyo estos funcionan de forma habitual pero no los vemos en pantalla. La otra alternativa es que 
para cada nodo se abre una consola, este modo tiene como objetivo permitir a usuario interactuar dentro de los nodos de forma manual.

En términos de visualización de los nodos, la herramienta permite habilitar dos tipos de animaciones, una basada en Python (Pyviz) que se muestra on line a la simulación. La otra alternativa está basada en NetAmin y es offline. Esta última genera un archivo XML que es interpretado por el NetAnim.

Para la configuración de los parámetros de DTN, la herramienta soporta la especificación por medio de una script de configuración en un archivo de texto.

Las especificaciones de la red están definidas por un archivo de programación en C, donde están descritas todas las instrucciones para la simulación con Ns-3. 


\subsection{Experimentos y evaluación}

\section{Escenario monitoreo de granja agrícola remota}

\section{Primer caso de uso: Generación de capacidades}

Este escenario permite valorar el aprovechamiento de la característica de movilidad de la mula, como medio de diseminación y descubrimiento de datos en un contexto de redes oportunistas. Durante el evento de movilidad, la mula puede tener contacto con dispositivos cluster head. Estos dispositivos tienen mayores recursos de hardware (en comparación con sensores y actuadores), que le permiten desempeñar tareas de coordinador y sumidero de datos. Estas características le facultan para ofrecer recursos propios y subyacentes en las WSAN (sensores, actuadores, datos, etc.).

Los roles involucrados en este caso incluyen a la mula de datos, y los dispositivos cluster head. Las funcionalidades utilizadas por los dispositivos son: la escucha de beacom, recepción de peticiones a nivel de servicio, respuesta a peticiones de información de recursos. Por su parte, la mula se anuncia mediante beacom, invoca peticiones de datos, y luego gestiona la información para generar capacidades.

El algoritmo sujeto de la validación es el de descubrimiento, el cual además de reconocer dispositivos y recursos, también posibilita la creación de capacidades que puede utilizarse para resolver asíncronamente componentes de los servicios.

Para la evaluación de este caso de uso en el escenario descrito, utilizaremos dos nodos. El nodo A, representa la mula de datos. Esta utilizará una trayectoria de movimiento fijo. En nuestro escenario dichos patrones son más predecibles, dadas las restricciones impuestas por las carreteras, límites de velocidad, rutas predefinidas, etc. Oportunamente, la mula tendrá la oportunidad de contactar con un dispositivo de tipo cluster head (nodo B), el cual asumimos que tiene almacenada la información de los sensores de la parcela, que ha almacenado durante un periodo de tiempo determinado. Adicionalmente tiene posibilidad de comunicación con los sensores de la WSAN. El nodo B, es un dispositivo de naturaleza estática.

Asumiremos una velocidad constante de $20 \mathrm{Km} / \mathrm{h}$, la cual es una velocidad razonable para una zona suburbana. Sin embargo, la velocidad puede ser variable puesto que el rol de la mula puede ser provisto por equipos que pueden controlar la velocidad, como es el caso de los drones. La interfaz de comunicación de ambos nodos es compatible con el estándar IEEE 802.11n, y su radio de cobertura es de 30 metros.

Este caso de uso está orientado por las siguientes preguntas:

1. ¿El algoritmo de descubrimiento procesa correctamente las capacidades?

2. ¿El algoritmo de generación de capacidades es capaz de procesar las capacidades, en un tiempo aceptable?

3. ¿Tamaño de la implementación del algoritmo permite escalar en escenarios de mayor densidad de dispositivos cluster head? 
Para responder a la primera pregunta, planteamos un escenario en donde los nodos A y B, tienen contactos que le permiten el intercambio de mensajes correspondientes a los datos de los recursos ofrecidos por el nodo B. Hemos repetido la misma simulación 120 veces, y registramos los resultados obtenidos por el algoritmo. Los resultados son comparados con respecto a la cantidad de recursos que de debieron haberse generado. La Figura 42, muestra los resultados de este experimento.

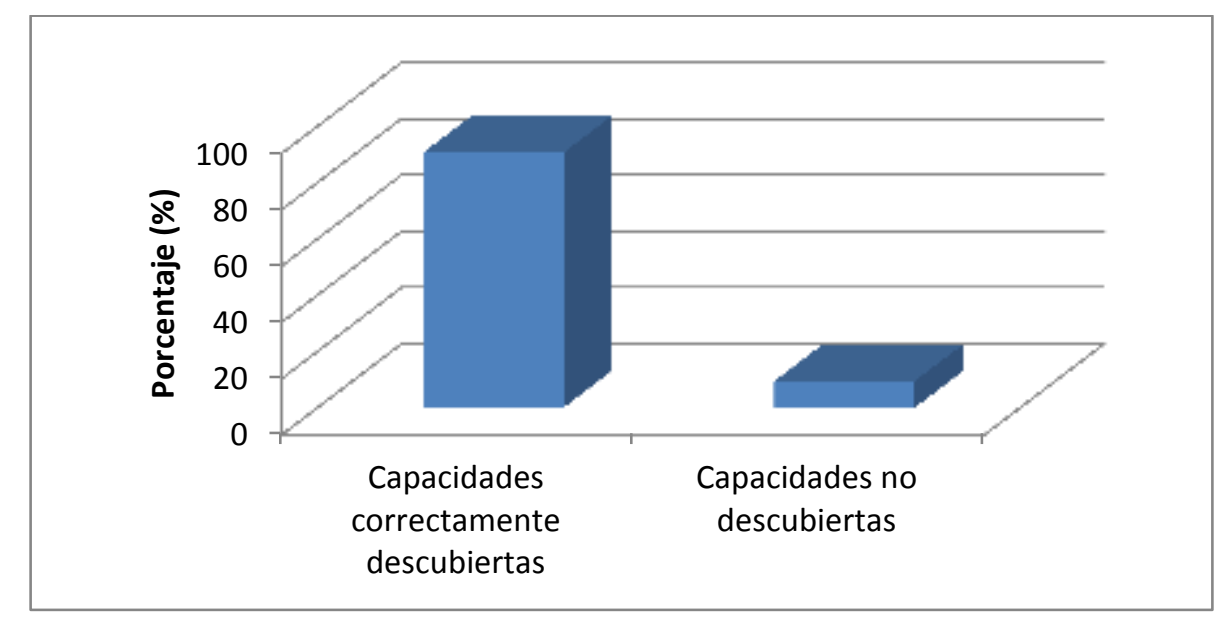

Figura 42: Descubrimiento de capacidades

Como se puede ver en el $90.58 \%$ de los casos, todos los datos de los recursos trasmitidos por el cluster head se registran apropiadamente como capacidades. Existen aproximadamente un $9.42 \%$ que no se llegan a registrar. Esto se debe a dos causas principales, la primera es que el nodo A, ya conozca el dispositivo, es decir, que tiene el nodo registrado en el repositorio de dispositivos. En el caso de que un dispositivo registrado previamente, sea enriquecido con nuevos recursos (sensores o actuadores), el algoritmo no será capaz de realizar un descubrimiento de todos los recursos disponibles. La segunda causa es que algunos recursos no hayan estado habilitados en el evento de descubrimiento, y posteriormente cambien su estado a disponible (ejemplo: por falta de energía).

En el caso de que la comunicación se interrumpa en el momento en que se está transfiriendo los bundles de datos con la información de los recursos disponibles, en el siguiente contacto de la mula, el algoritmo hace uso de las características DTN para recuperar el resto del bundle que hace falta, y con esto termina de crear las capacidades pendientes, esto permite garantizar la transferencia de datos y optimizar la ventana de tiempo de contacto.

Nunca habrá un recurso inexistente que sea registrado como una capacidad, porque el algoritmo trabaja únicamente con la información de los recursos existentes en los nodos coordinadores. 
Para responder a la segunda pregunta, simulamos un escenario de una WSAN con 50 sensores, asumimos que cada sensor está representado por una capacidad distinta. La mula pasa a unos 15 metros del cluster head. Este escenario se ejecutó 25 veces, y se calcularon los tiempos que tarda la mula en generar todas las capacidades de esos sensores. En la Figura 43, se puede observar dos curvas que representan la distribución probabilística para las dos alternativas que considera el algoritmo de descubrimiento, es decir, cuando descubre un nuevo dispositivo, y cuando ya conoce con antelación el mismo.

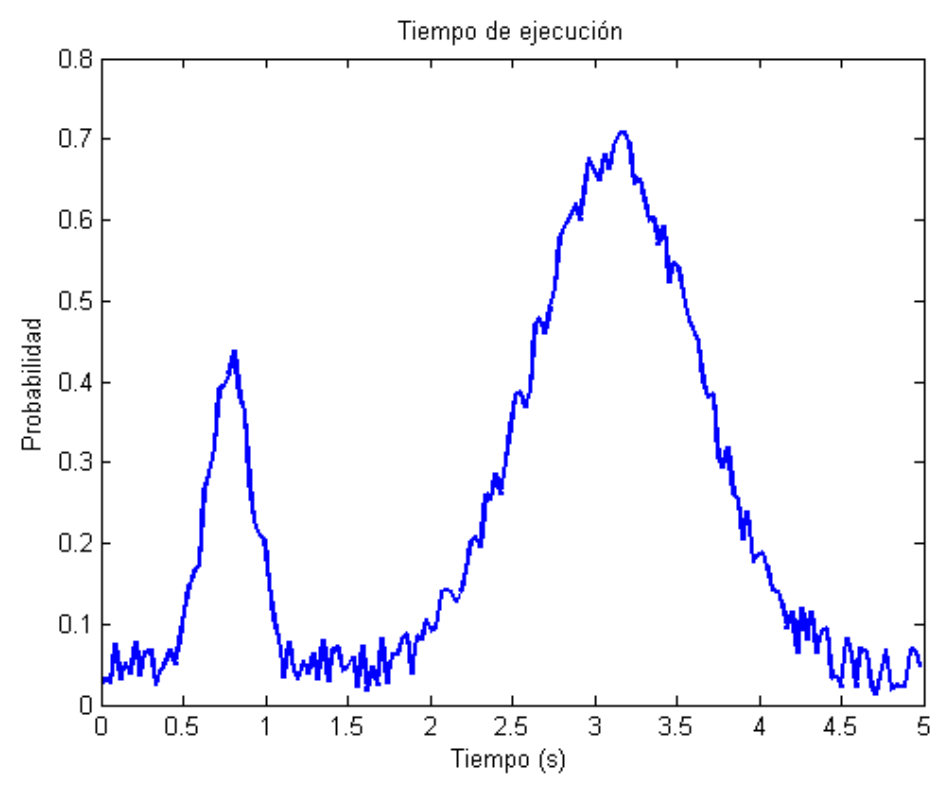

Figura 43: Tiempo de procesamiento de capacidades

La primera curva de la gráfica representa, la sección del algoritmo que considera operaciones de actualización únicamente. En este caso, el algoritmo tarda aproximadamente 0.70 segundos, con un margen de error máximo de \pm 0.5 segundos. Este tiempo es acotado producto de las pocas instrucciones de acceso a los datos que tiene que ejecutar para realizar las tareas de actualización.

El tiempo medio de procesamiento de nuevas capacidades es de 3 segundos, el cual está representado en la gráfica como la curva de distribución más elevada. Esta medida es producto de la mayor complejidad y cantidad de instrucciones en este flujo del algoritmo. El tiempo de acceso a los datos almacenados introduce un retardo importante que contribuye a aumentar el tiempo de procesamiento en esta sección del algoritmo. Esta distribución de probabilidad está acotada por un error aproximado de \pm 1.8 segundos.

Considerando que el tiempo de procesamiento máximo es de 4.8 segundos, podemos concluir que el algoritmo es capaz de procesar la información en unos tiempos aceptables, ya que estos tiempos están dentro del rango de lo que se espera sea la ventana de tiempo de transferencia de datos durante el contacto entre la mula y una WSAN. 
Para responder a la tercera pregunta, hemos configurado un experimento, para calcular el tiempo medio que demora la mula en registrar las capacidades en WSAN con densidad en el rango de 0 y 200 cluster head.

En este escenario de múltiples dispositivos, para cada uno se repetido la simulación 12 veces, hemos calculado el tiempo medio de generación de capacidades en 6.55 segundos. Para cada dispositivo se el tiempo medio de registro de la mula, y el tiempo medio de la ventana de tiempo de transferencia de datos, se pueden observar en la Figura 44 .

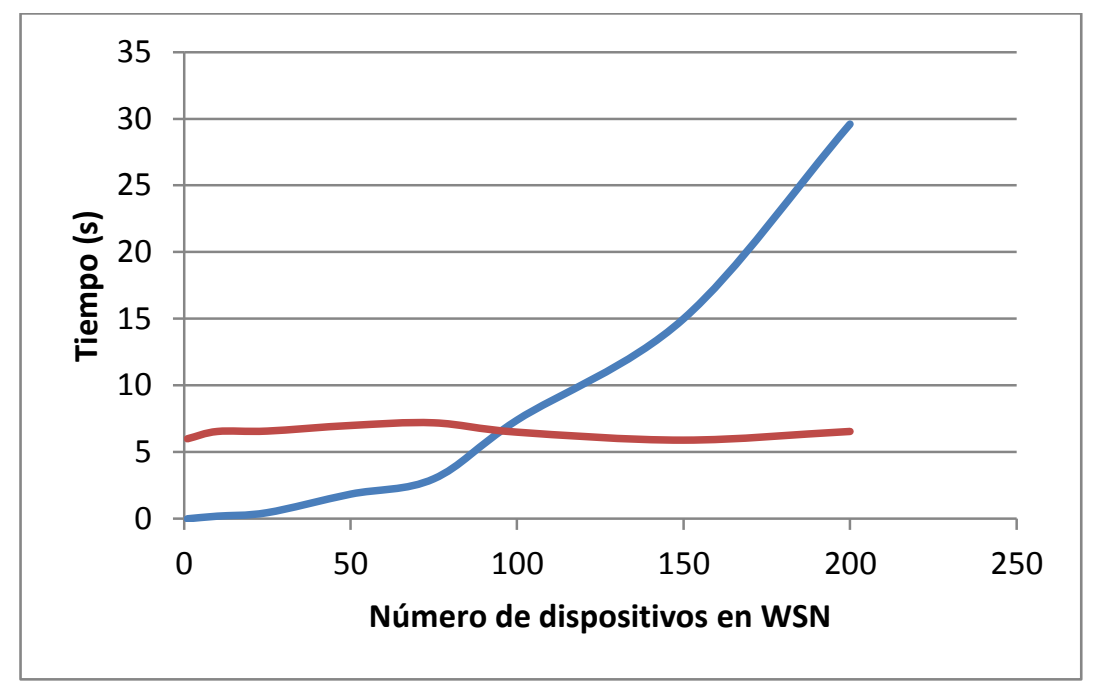

Figura 44: Escalabilidad del algoritmo de descubrimiento

La línea roja horizontal representa el tiempo medio de contacto entre el cluster head y la mula, es decir, la ventana de tiempo de transferencia de datos. Este valor se mantiene bastante estable con respecto a la densidad de dispositivos.

El tiempo medio de necesario para la generación de capacidades, por parte de la mula, está representado por la línea azul. Esta tiene una tendencia cuadrática, debido a que hay condiciones en el algoritmo que dependen la cantidad de capacidades, con lo que se refleja más o menos como un $\mathrm{N}^{2}$.

La intersección indica que la mayor cantidad de dispositivos que pueden ser atendidos por el algoritmo en un solo contacto es aproximadamente de 90 dispositivos o coordinadores de WSAN. Esta cobertura es suficiente para los escenarios típicos de aplicación del algoritmo, por lo tanto, podemos decir que el algoritmo es suficientemente escalable para el contexto objetivo. 
Segundo caso de uso: Resolución de componentes

Este caso permite evaluar las características del algoritmo de MatchMaking. Este algoritmo tiene como función principal la resolución de los componentes de los servicios, mediante una estrategia de matching, que considera las condiciones básicas y opcionales especificadas en el perfil del servicio, y por otro lado, toma en cuenta las restricciones definidas por las características de los recursos. Este proceso utiliza un matching sintáctico, puesto que el semántico requiere de la disponibilidad de equipos con mejores características de hardware y software, que permitan las complejas operaciones sobre metadatos. Estas características no son habituales en entornos de movilidad, y específicamente en el escenario de estudio. Un matching semántico genera un retardo considerablemente largo en el procesamiento, para ejecutarse de forma aceptable en un escenario de movilidad que incluye la resolución continua y dinámica de componentes.

Para realizar la valoración de este caso de uso, hemos simulado un escenario en el que se realizan dos tipos de procesamiento de matching, estos incluyen el sintáctico y el semántico. En ambos casos se evaluaron con diferentes cantidades de servicios, en un rango de 0 a 150. En la Figura 45, se puede observar el rendimiento del algoritmo en sus dos versiones de matching.

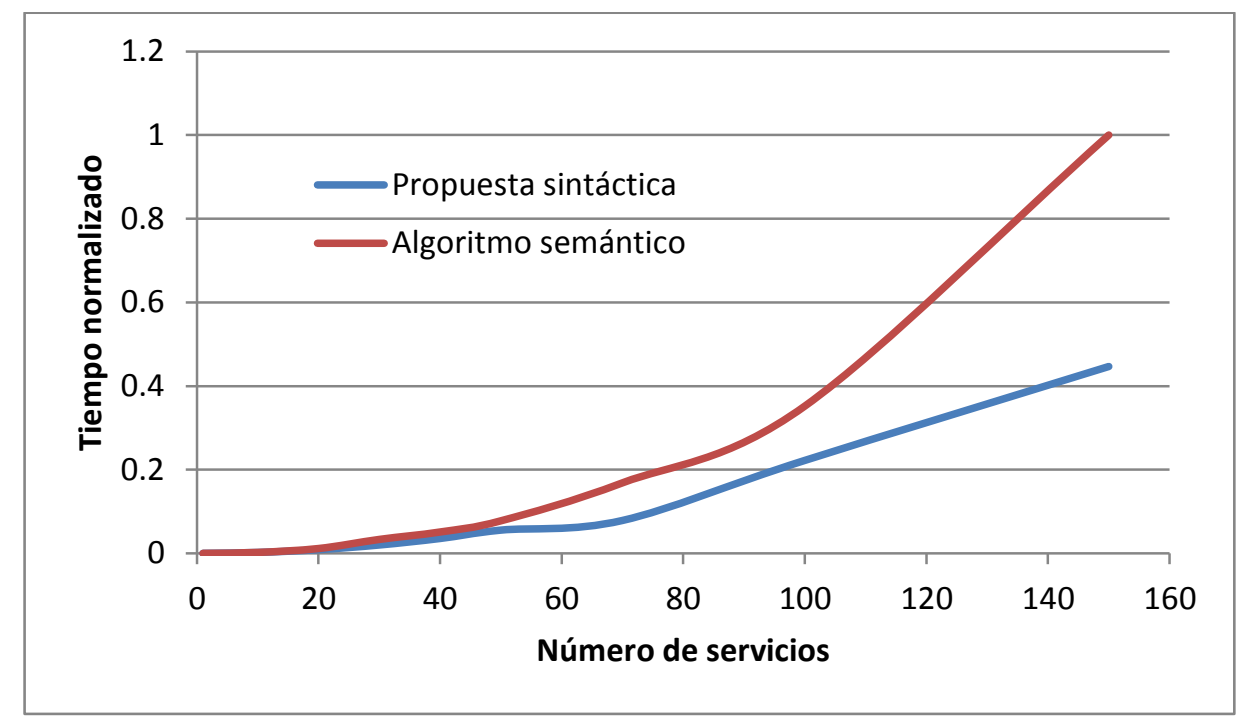

Figura 45: Evaluación del MatchMaking

Aun cuando el matching semántico evidentemente introduce mayores retardos, hay una primera parte donde la diferencia no es acusada, es decir, ambas estrategias resultarían con igual rendimiento. Para escenarios que involucran más de 60 servicios, se podría asumir un retardo mayor si se precisa de las funciones de procesamiento semántico. A partir de 67 servicios aproximadamente, se incrementa significativamente el tiempo necesario para el procesado semántico, por lo tanto no es recomendable utilizarlo en el escenario de aplicación en estudio. 


\section{Escenario de automatización de granja agrícola remota}

Primer caso de uso: Desplazamiento de servicio

La función principal de este caso de uso es comisionar la ejecución del servicio en la mula, como se ha descrito en la sección 9.2.1. Este caso ilustra la versatilidad de la arquitectura, permitiendo el soporte para la delegación de servicios y su ejecución remota. Esta característica permite la ejecución del servicio en el sitio en donde se encuantren los recursos más adecuados para su correcta realización. Esto posibilita alcanzar mayores niveles de optimización en los tiempos de ejecución, y rentabilizar el tiempo de contacto con los recursos remotos.

El algoritmo que interviene en este caso de uso es el de delegación de servicio, que se ha descrito en la sección 7.3. Este algoritmo está orientado básicamente por los patrones de conducta de la movilidad de la mula, es decir, se consideran dos tipos de mulas, que incluyen las deterministas y las indeterministas. Esta característica se determina dinámicamente por la función probar determinista (Algoritmo 8). Este algoritmo define el determinismo en función de dos parámetros principalmente, el primero es la cantidad de contactos de la mula con el framework de servicios, representado por $\mathrm{N}$ en el algoritmo. Este valor se calcula y acumula con cada notificación de la mula.

El valor asignado a $\mathrm{N}$ es fundamental en la efectividad de la ejecución de los servicios sobre mulas. Este parámetro influye significativamente sobre el cálculo del factor de confianza de la mula y la clasificación de la mula como determinista o indeterminista. Para determinar los valores óptimos para este factor, hemos realizado un experimento para determinar la probabilidad de ejecución de los servicios en función de la cantidad de notificaciones de la mula ante el framework de servicios. Los resultados se pueden observar en la Figura 46.

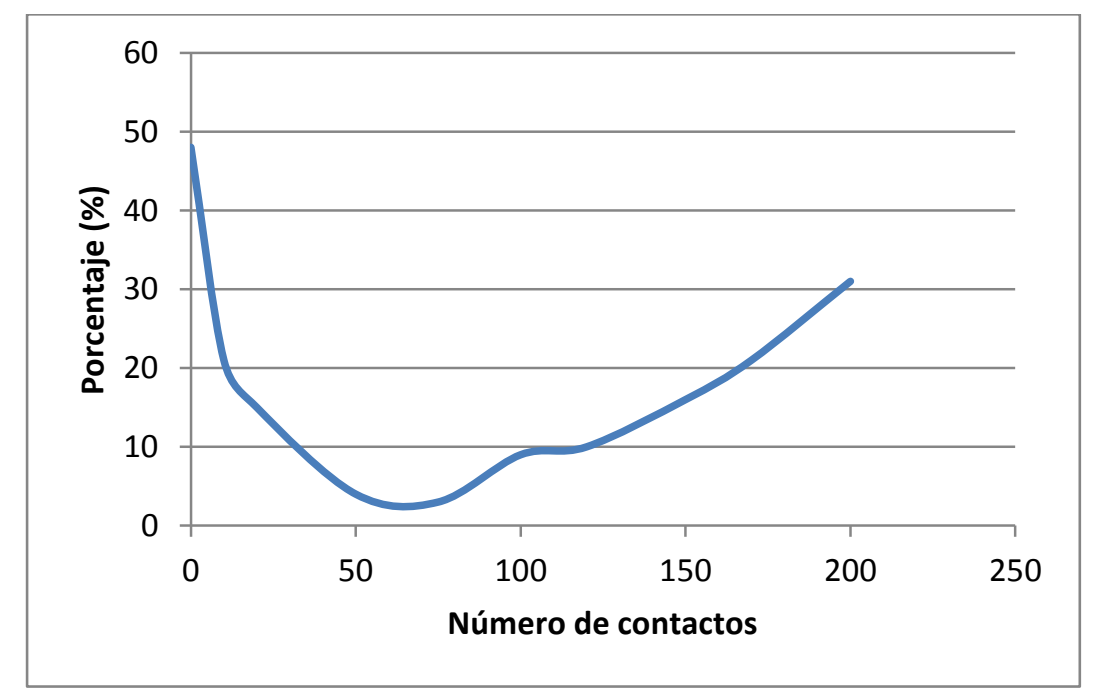

Figura 46: Probabilidad de ejecución según cantidad de contactos 
El trazo inicial dela gráfica (parte izquierda), en el rango de 0 a 20 contactos, la probabilidad de que un servicio no se ejecute es alta, puesto que las mulas se convierten en deterministas rápidamente. Es decir, aunque sean mulas deterministas no son confiables en sí, ya que el número de contactos que se establece es muy pequeño, y no permite tener mayor grado de certeza en sus patrones de conducta.

En la parte baja de la gráfica se puede observar que cuando el número de contactos esta aproximadamente entre 30 y 100, es cuando hay mayor probabilidad de que el servicio se ejecute, siendo esta la zona óptima de ejecución de servicios.

La zona de la derecha, después de los 110 contactos aproximadamente, existe una tendencia creciente en la probabilidad de no ejecución de los servicios. Esto se da porque se exige tantos contactos que puede ocurrir que transcurra mucho tiempo para satisfacer tales condiciones.

El otro factor, simbolizado por la variable $X$ en el algoritmo, representa un rango de precisión aceptable para los valores de las desviaciones de los retardos, y es definido por el usuario. El siguiente experimento, evidencia cual es el comportamiento de las probabilidades de ejecución, en relación a los tiempos entre contactos, o notificaciones de la mula ante el framework de servicios. En la Figura 47, se pueden observar los resultados de este experimento.

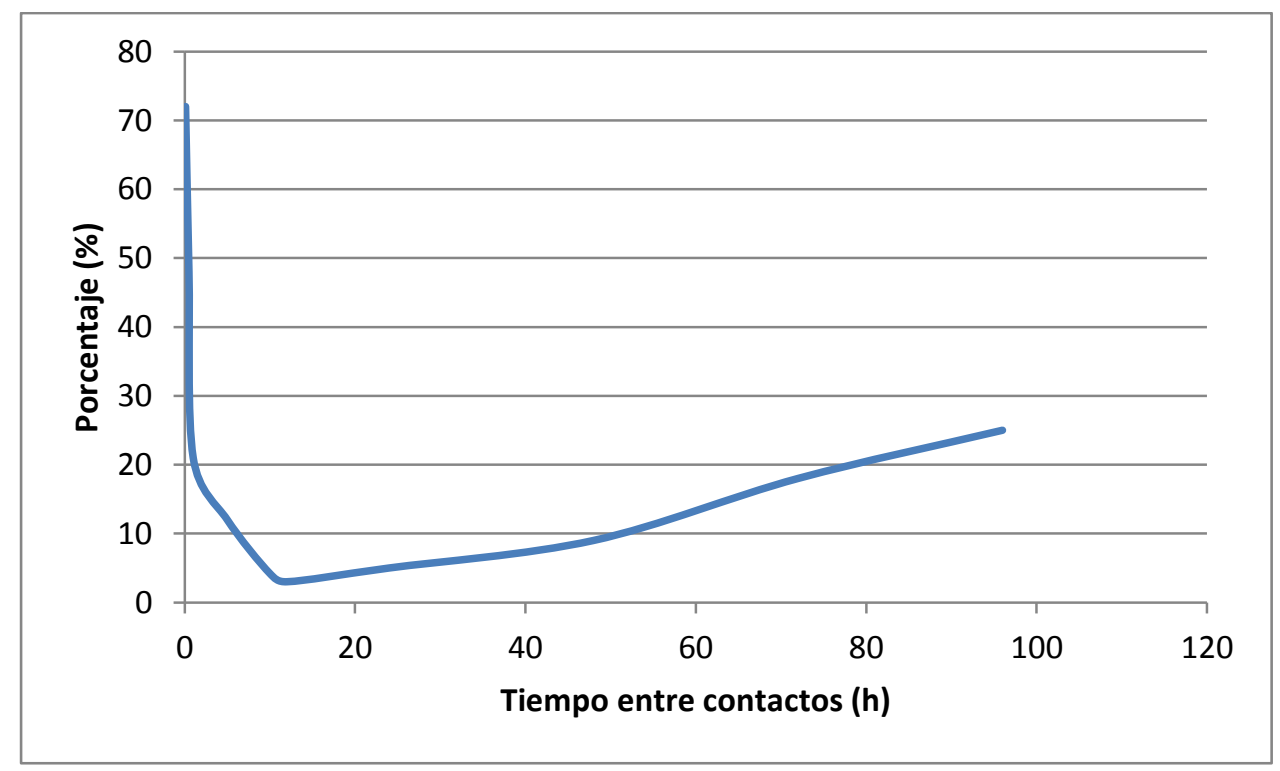

Figura 47: Probabilidad de fallo en la ejecución según tiempo entre contactos

La zona inicial de la gráfica (izquierda), se da cuando hay poco tiempo entre contactos. En escenarios de la vida real es difícil encontrar mulas con estos tiempos entre contactos, que resulten útiles para ejecutar algún servicio, por lo tanto, existe poca probabilidad de que se ejecuten los servicios. En parte más baja de la curva, entre 5 y 48h, es un tiempo entre contactos aceptablemente bueno para la ejecución de servicios, es decir, existe menor probabilidad de no ejecución. Sin embargo, hacia la 
derecha cuando el tiempo entre contactos se incrementa considerablemente, la probabilidad de no ejecución aumenta, debido al incremento gradual en la latencia de ejecución de los servicios.

Las garantías de que la delegación termine con una ejecución exitosa de los servicios, depende de varios factores incluyendo los anteriormente analizados. Asumiendo que los valores de $\mathrm{N}$ y X, están en el mejor de los casos, dentro los parámetros adecuados anteriormente estudiados, realizamos una simulación de ejecución de servicios, donde el énfasis está enfocado en la delegación y ejecución remota de los servicios. Para este experimento, consideramos mulas deterministas e indeterministas. Los resultados se pueden observar en la Figura 48, esta gráfica muestra las probabilidades de ejecución exitosa bajo condiciones ideales según el algoritmo de delegación.

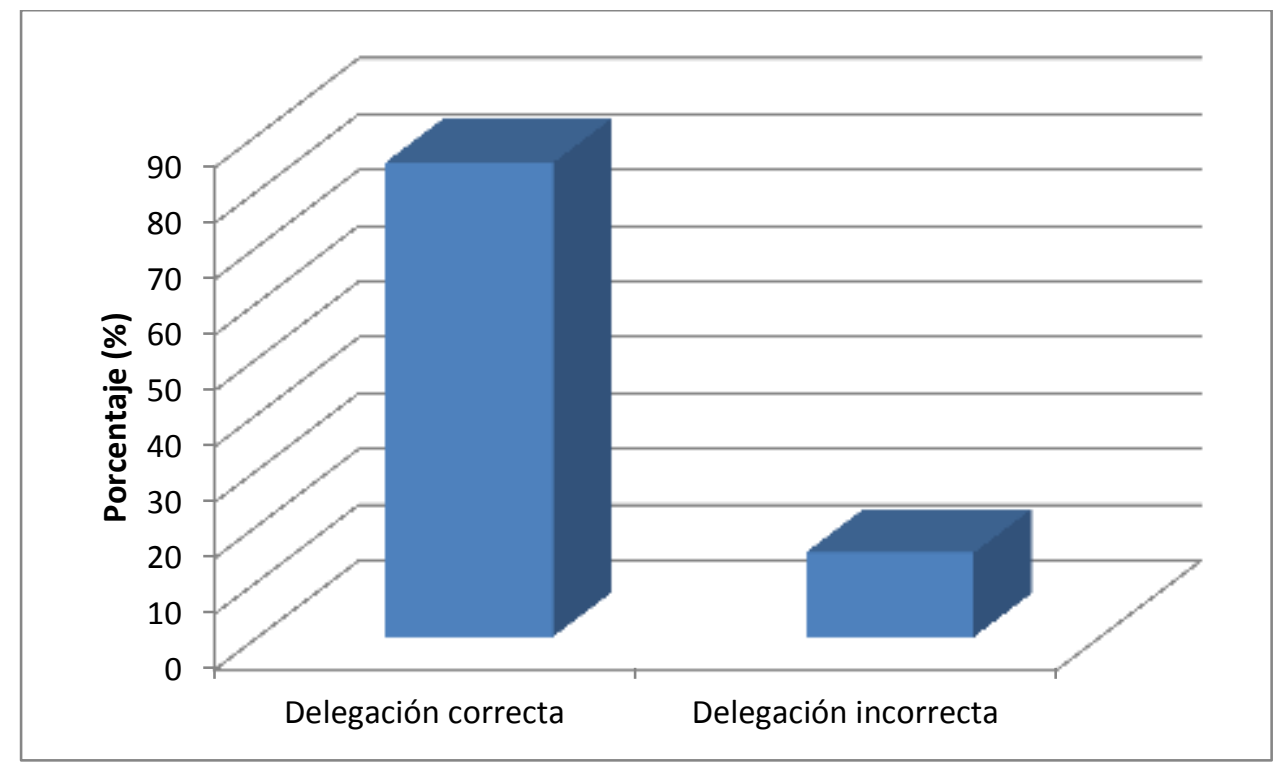

Figura 48: Probabilidad de ejecución exitosa

Como es evidente, según la gráfica anterior, la mayoría de los servicios se ejecutan de forma satisfactoria, con una efectividad de $85 \%$. Sin embargo, este algoritmo no es infalible a eventos comunes que pueden darse en el escenario de estudio. Las causas de que el 15\% de las delegaciones sean fallidas se deben principalmente a estas causas:

Se delega en mula indeterminista, pero sus patrones de movilidad son aleatorios y no es capaz de ejecutar las capacidades para resolver el servicios, por no vuelve a tener contacto con los dispositivos asociados a las capacidades que debe ejecutar.

Fallo de un recurso asociado a una capacidad, con la cual se creó un matching, y luego el servicio no puede ser nuevamente delegado a otra mula, hasta que se detecte el fallo en la ejecución. 
Segundo caso de uso: Ejecución de servicios de control

Este caso de uso considera todos los roles dentro de la arquitectura, incluyendo los actuadores. En la ejecución de servicios de control intervienen todos los elementos componentes de la arquitectura propuesta.

Los experimentos se han repetido 100 veces, habilitando y deshabilitando la delegación de servicios. El servicio de control en la nube implica una primera comunicación nube-mula-sensor, luego en sentido contrario, y finalmente una tercera indicando que realice una tarea. Este esquema de comunicación introduce un retardo prolongado en la ejecución del servicio.

Cuando el servicio se ejecuta en la mula, se requiere mucho menos tiempo para ejecutarlo. Esta facilidad permite que los servicios se ejecuten más cerca de los participantes, reduciendo considerablemente el retardo en la ejecución.

El tiempo necesario para ejecutar un servicio de control, cuando está delagado en la mula, es mucho menor que el necesario para ejecutar el servicio de control cuando se ejecuta desde la nube.

La Figura 49, muestra una descripción grafica de la distribución de los resultados de los experimentos. Es evidente la contribución del proceso de delegación en los tiempos de ejecución de los servicios. Los servicios no delegados tienen un tiempo medio de ejecución aproximado de 6 horas. Sin embargo, esos mismos servicios delegados tienen un tiempo de ejecucón medio de 1 hora.

Ejecución en la nube

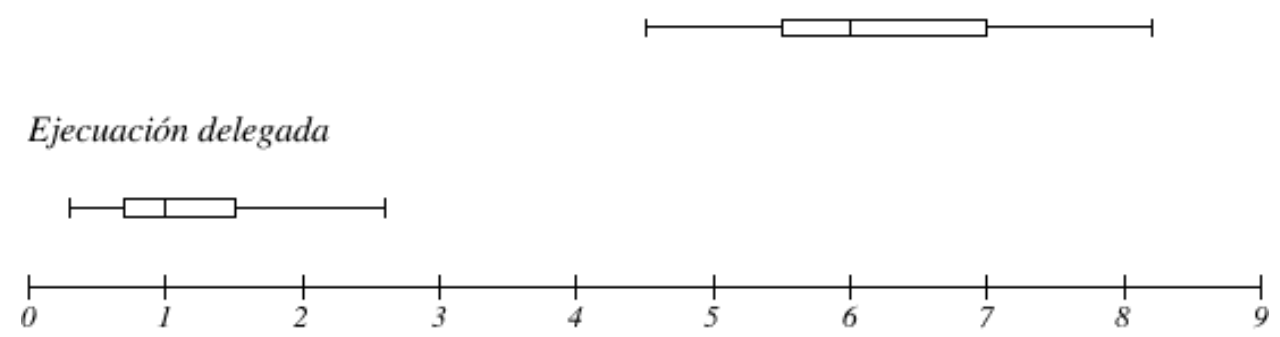

Figura 49: La distribución estadística de los resultados de la ejecucíon

Tercer caso de uso: ejecución de servicios a bajo nivel en las redes DTN

Este caso de uso que analizamos considera la ejecución de servicios a bajo nivel en las redes DTN.

La arquitectura desarrollada ha consistido en un dispositivo DTN, 15 cluster head y 110 sensores y actuadores (distribuidos de forma homogénea entre los cluster head). Los cluster head se conectaron al dispositivo DTN a través de un enlace Bluetooth. Una solución basada en XML se empleó como modelo de datos para esta comunicación 
Dipositivo DTN - cluster head. La conexión entre los sensores y el cluster head se resolvió utilizando el protocolo IEEE 1451. Todos los costes definidos por los administradores se fijaron a un valor unitario $q_{a d m i n}=1$. Las contribuciones para el cálculo de coste se consideran independientes, por lo que la función de coste elegida $f_{\text {cost }}$ se ha definido como la media aritmética.

El coste debido a las consideraciones hardware se entiende como la media aritmética de dos indicadores: el consumo de batería $\psi_{\text {bat }}$ y la memoria ocupada $\psi_{\text {mem }}$ (8-9). Todos los servicios del sistema se han definido como atómicos y no-geográficos.

$$
\begin{gathered}
\psi_{\text {bat }}=\frac{\text { reduction in the battery charge }}{\text { total battery capacity }} \\
\psi_{\text {mem }}=\frac{\text { occupied memory }}{\text { total installed memory }}
\end{gathered}
$$

Se han realizado dos experimentos. Durante el primer experimento, las conexiones entre componentes se consideraron permanentes, y se forzaron pérdidas de paquetes en el enlace Bluetooth de forma progresiva. El tiempo de ejecución fue monitorizado para la estrategia propuesta de gestión individual.

En el segundo experimento se consideraron conexiones no permanentes. Se monitorizó el número de comunicaciones exitosas y el tiempo de ejecución empleando ambas estrategias (gestión individual y por grupos). La Figura 50, representa los resultados del primer experimento.

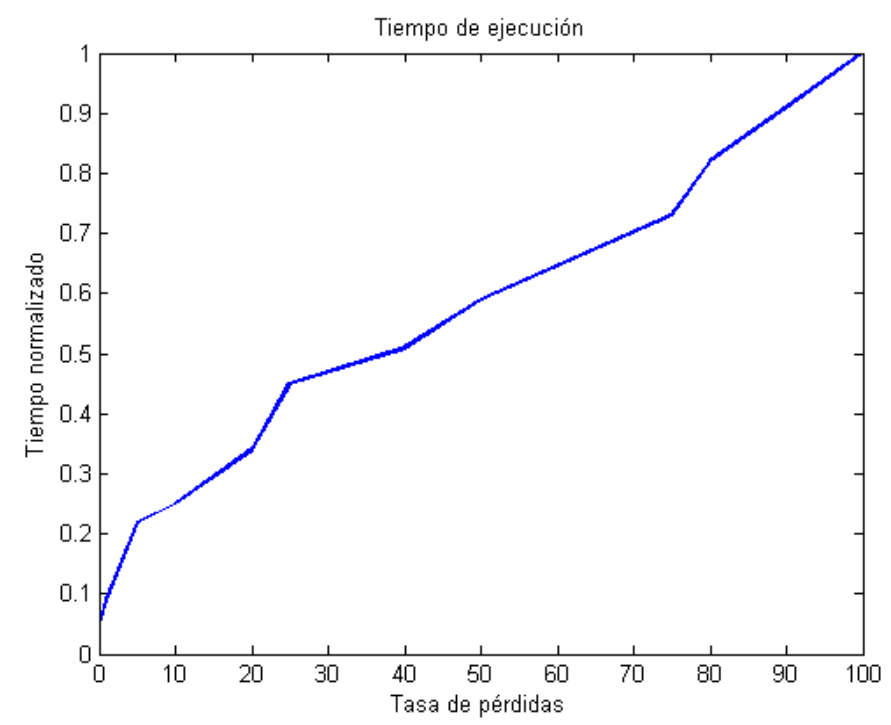

Figura 50: Tiempo de ejecución resultante del primer experimento

Como puede verse, el tiempo de ejecución crece de forma lineal cuando aumenta la probabilidad de pérdida de paquete. Esto indica que el número de interacciones necesarias para realizar una ejecución exitosa también crece. La variación es, como se indica en el modelo teórico, cercana a los dos órdenes de magnitud. En situaciones 
normales (en redes con congestión mínima) el tiempo de ejecución es bajo (cercano a 0.1 en las unidades temporales normalizadas utilizadas en el experimento).

La Figura 51, muestra los resultados del segundo experimento comparando la evolución del tiempo de ejecución obtenido utilizando la estrategia de gestión individual y la estrategia de gestión de grupos. Los grupos de cluster head consisten en 5 unidades. Como se ha visto en el modelo teórico, el tiempo de ejecución es mayor que en la estrategia de gestión de grupos (hasta 7 veces mayor en el caso peor). Sin embargo, como indica la figura, la evolución es constante y lineal. Esto es importante para escenarios donde las conexiones son no-permanentes (como en el caso de estudio de este trabajo de tesis). Este tipo de conexiones puede tolerar un tiempo de ejecución mayor, pero las altas variaciones no se soportan de forma tan adecuada.

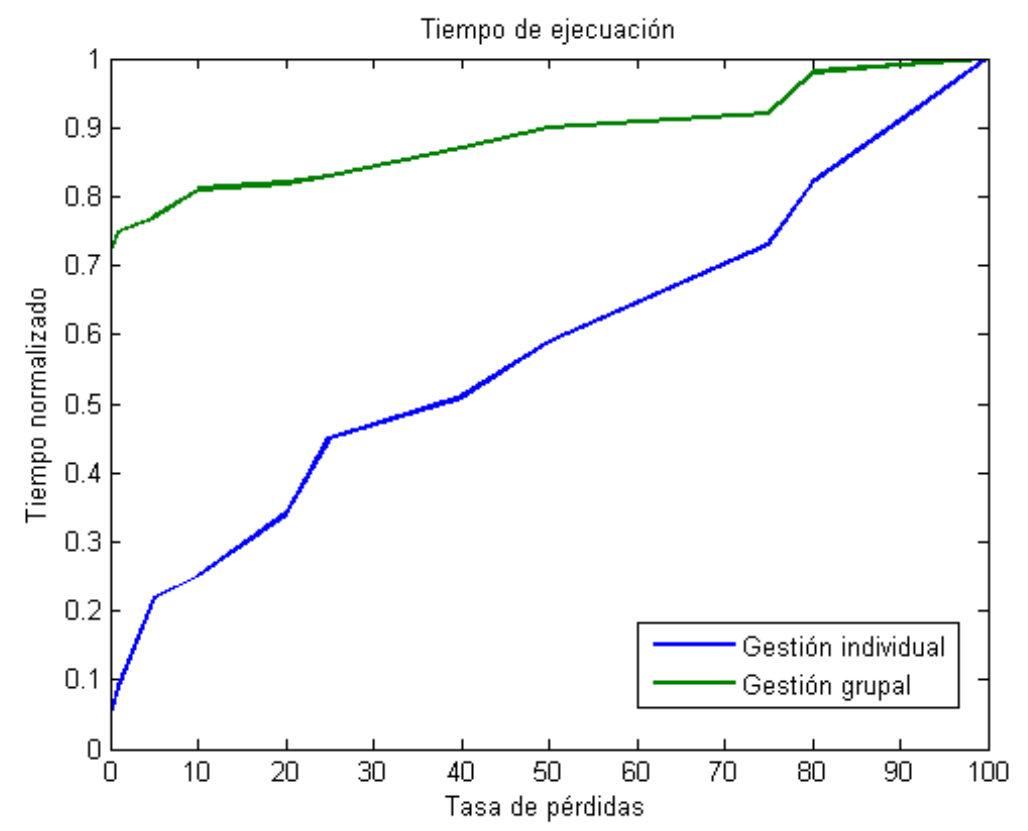

Figura 51: Tiempo de ejecución resultante del segundo experimento

Por último, la Figura 52 muestra la mejora en el número de ejecuciones satisfactorias en el caso de la gestión individual y por grupos. Como puede verse existe una mejora en este último caso de casi un $50 \%$. 


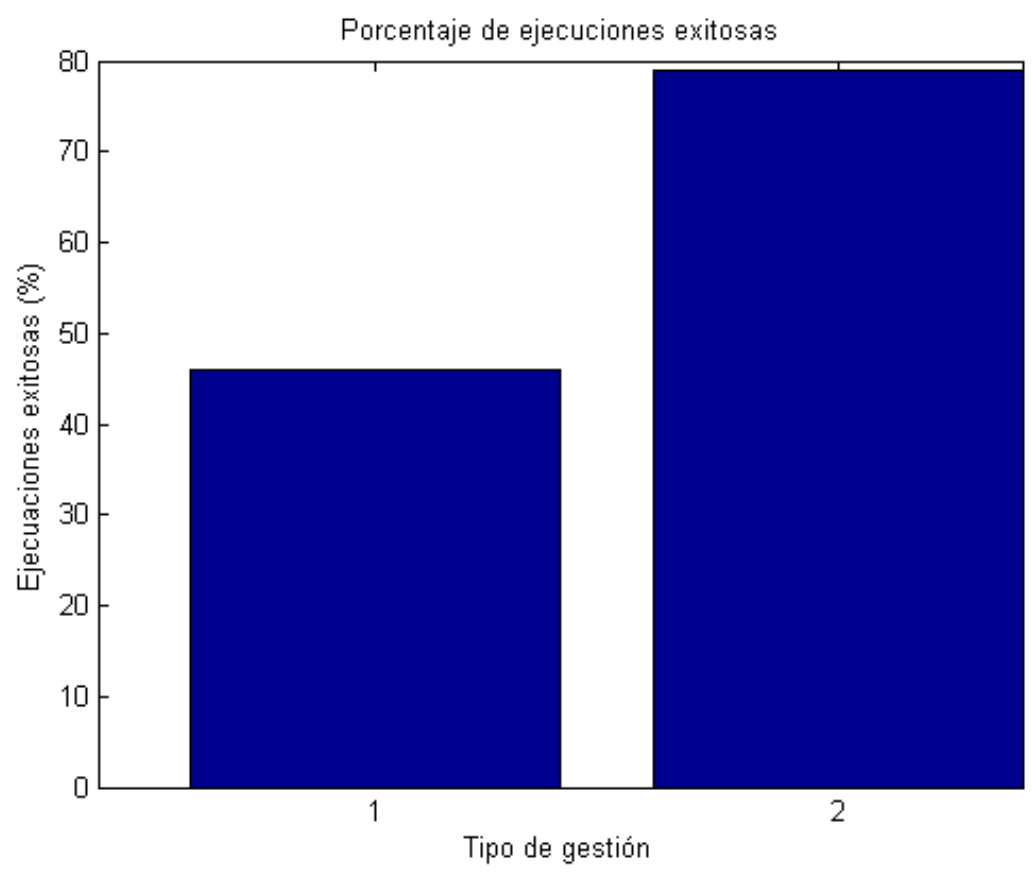

Figura 52: Comparativa de porcentaje de ejecuciones satisfactorias

\subsection{Conclusiones}

El primer caso de uso de generación de capacidades modela el contacto incial entre una mula y un dispositivo. Como podemos ver en la experimentación, cuando el canal de datos queda establecido, la mula puede generar peticiones de información sobre los recursos disponibles en el dispositivo. Este proceso de intercambio de mensajes se produce mientras la mula sigue en movimiento, con lo cual es de vital importancia optimizar al máximo la ventana de tiempo disponible para el intercambio de datos entre la mula y el dispositivo.

En el segundo caso de uso de resolución de componentes, describe el evento de comunicación entre la mula y el framework de servicios en la cloud. Se realiza bajo condiciones de movilidad de la mula, con lo cual la velocidad del proceso y las comunicaciones son vitales, para optimizar el tiempo de contacto. Se concluye que aún cuando el matching semántico evidentemente introduce mayores retardos, hay una primera parte donde la diferencia no es acusada, es decir, ambas estrategias resultarían con igual rendimiento. Sin embargo, a partir de 50 servicios aproximadamente, se incrementa exponencialmente el procesado semántico, por lo tanto no es recomendable utilizarlo en el escenario de aplicación en estudio.

El tercer caso de uso, de desplazamiento o delegación de servicio permite que los usuarios puedan crear servicios distribuidos, estos servicios pueden ser remitidos a una mula, para su posterior ejecución en forma remota. De la experimentación se puede extraer que la mayoría de los servicios se ejecutan de forma satisfactoria, con una efectividad de $85 \%$. Sin embargo, el algoritmo de delegación no es infalible a 
eventos comunes que pueden darse en el escenario de estudio. Las causas de que el $15 \%$ de las delegaciones sean fallidas

La validación experimental del cuarto caso de estudio (ejecución de servicios de bajo nivel) nos muestra que la estrategia de gestión individual proporciona un tiempo de ejecución muy breve en el mejor de los casos, pero este tiempo crece de manera rápida cuando la plataforma hardware presenta pérdidas en el nivel de comunicaciones. En este caso, la gestión por grupos genera unos tiempos de ejecución más estables, a costa de que el tiempo de ejecución se incremente respecto a la gestión individual. 


\section{Conclusiones y trabajos futuros}

El trabajo de tesis está motivado por la necesidad de disponer de modelos específicos de servicios que contemplen los requerimientos identificados en el dominio de las WSAN desplegadas en zonas aisladas. El desarrollo una plataforma integrada, que permita ofrecer un framework de gestión de servicios basados en las capacidades proporcionadas por las WSAN, mediante una interfaz basada en tecnologías cloud, representa un aporte significativo para la investigación, ciencia, industria, agricultura y la comunidad de usuarios de WSAN en general.

Esta sección resume las contribuciones del trabajo de tesis y justifica la validación de las contribuciones comprobando que cumplen los objetivos definidos y asociados a cada una de ellas. Se discute además la novedad de utilizar la contribución propuesta comparando su aplicación frente a otras soluciones del estado del arte.

Finalmente se describe un conjunto de trabajos futuros relacionados con los objetivos de investigación de la tesis, abordando las limitaciones de las soluciones para una posible generalización, aplicación a otros entornos, o adaptación a cambios tecnológicos. 


\subsection{Conclusiones}

En el ámbito de las redes inalámbricas de sensores con comunicaciones limitadas, la recuperación, procesamiento y posterior transformación en servicios disponibles para la comunidad científica, sigue sin tener solución, por lo que ésta se considera una necesidad que no ha sido resuelta de forma adecuada. En este trabajo de tesis de justifica la necesidad de disponer de una alternativa eficiente para la creación, despliegue y ejecución de servicios en ambientes de redes de sensores distribuidas.

Para resolver esta limitación, en el ámbito del acceso a la comunicación de información, se propone como objetivo principal el diseño de una solución para la ejecución de servicios sobre redes de sensores en entornos remotos, utilizando las características de los protocolos de las DTN.

Debido a este propósito se determina que es necesario el estudio de tecnologías de redes de sensores y actuadores, desde el punto de vista del conformado de redes heterogéneas, comunicadas a través de protocolos No-IP como ZigBee, Z-Wave, Insteon y Wavenis, protocolos basados en IP como 6LoWPAN, middlewares de comunicaciones para WSNs como RUNES, REMOWARE, LooCI, etc, y otras tecnologías de alto nivel para mejora la flexibilidad, interoperabilidad y brindar soporte a los distintos tipos de aplicaciones emergentes. En este último caso se estudian los protocolos MQTT, MQTT-SN y CoAP, como principales exponentes de las tecnologías de capas superiores.

La recolección de datos en entornos de WSAN remotas se aborda desde la problemática de la ausencia de enlaces de comunicación permanentes de extremo a extremo. Este problema se aborda en el estudio del estado del arte desde la perspectiva de las soluciones ad-hoc, que generalmente no consideran las restricciones propias de los dispositivos con recursos limitados, ni la necesidad de arquitecturas y tecnologías estándar, y de las soluciones basada en nodos móviles con capacidades DTN, que permitan ofrecer soporte a la comunicación entre las WSAN aisladas y una infraestructura de red con servicio continuo de comunicación, objetivo abordado en las contribuciones del trabajo de tesis.

Finalmente se requieren contribuciones a nivel de aplicación, que han sido estudiadas en el campo de las IoT y las comunicaciones M2M, analizando de forma particular las contribuciones en estos campos, a los procesos de resolución, delegación y ejecución distribuida de servicios.

- Este trabajo de tesis contribuye a la definición de una arquitectura de referencia para la ejecución distribuida de servicios en redes inalámbricas con infraestructura limitada. Esta arquitectura soporta el desarrollo de servicios móviles en el contexto de las WSAN distribuidas y la ejecución de servicios persistentes ante largos periodos de tiempo en espera, sin pérdida del estado de ejecución de los mismos. Las descripciones física y funcional 
de la arquitectura definen las capas de aplicación, servicios, DTN y dispositivos, de mayor a menor nivel de abstracción.

La complejidad de la interacción propuesta debido a las limitaciones de la ejecución distribuida en entornos disruptivos hace necesario prestar una especial atención al modelo de servicio utilizado en el trabajo de tesis.

- Se propone, por tanto, un modelo de servicio tolerante, como elemento software que realiza varias transiciones durante su ciclo de vida, y cuyo estado es persistente ante retardos. Este servicio viene soportado por su ciclo de vida, definido como un conjunto de procesos y de relaciones entre procesos: Creación, Búsqueda, Adaptación, Clasificación, Publicación, Ejecución y Verificación. El modelo de servicios se define en lenguaje UML y el ciclo de vida y los procesos que intervienen se definen en SPEM.

El conjunto de procesos que constituyen el ciclo de vida de servicio permiten su ejecución en entornos distribuidos y disruptivos. Para conseguir una distribución y ejecución eficiente es necesario dotar a los elementos que constituyen la red de comunicaciones, de inteligencia suficiente para ejecutar estrategias para cada uno de los procesos definidos anteriormente.

- Como contribución principal en este ámbito, se propone un modelo de ejecución de servicios y se realizan aportaciones a los procesos de resolución dinámica de componentes, reconocimiento oportunista de recursos, descubrimiento de dispositivos y matchmaking. La puesta en marcha de los algoritmos definidos en la Sección 6 nos permite validar el comportamiento eficiente de la red.

- Adicionalmente, se propone un mecanismo de delegación de servicios para aproximar la ejecución de servicios a los elementos participantes y así obtener mayor eficiencia en la ejecución. Se consideran los casos en los que la movilidad de las mulas responde a patrones fijos (delegación determinista), y en los que las mulas no tienen una conducta de movilidad estable o predecible (delegación indeterminista). Este mecanismo se aplica a un escenario de validación relacionado con la provisión de riego en una granja inteligente de cultivos.

Ya que las redes de sensores pueden encontrarse en lugares aislados y las comunicaciones son a través de elementos móviles o mulas, con comportamientos muchas veces indeterministas, se necesita una mayor versatilidad en el comportamiento a nivel de comunicaciones para dichos sensores. Para conseguir una mayor versatilidad en la configuración y programación de dispositivos en tiempo de ejecución, se realiza un análisis de los mecanismos de autoconfiguración y autoorganización de las redes de sensores.

- Se propone en este ámbito una estrategia de ejecución de servicios sobre nodos en redes de sensores y actuadores, que consiste en un proceso de 
monitorización de hardware y dos estrategias de gestión de las órdenes de invocación de servicios de forma que la combinación de dichas estrategias nos permita minimizar el tiempo de ejecución, y establecer valores de calidad de servicio estables con el fin de optimizar la calidad de los servicios ejecutados.

Por último se proponen un conjunto de escenarios de aplicación, entre los que desatacan el de monitorización de granja agrícola remota, y el de automatización de granja agrícola remota. Estos escenarios se analizan para la obtención de un conjunto de requisitos funcionales y no funcionales, que se utilizan para el diseño del entorno de simulación. La generación de experimentos y la evaluación de los mismos, permiten comparar la solidez de la investigación presentada y las mejoras que suponen las contribuciones planteadas.

\subsection{Trabajos futuros}

Esta sección describe los trabajos futuros planificados a corto y medio plazo, relacionados con las contribuciones de la tesis.

La principal extensión de este trabajo estará relacionada con la puesta en práctica de las soluciones aportadas en esta tesis sobre entornos reales de ejecución de servicios distribuidos. De esta forma podrán compararse los resultados generados a través de las simulaciones realizadas utilizando el software Ns-3 y la extensión de ejecución de aplicaciones y servicios basada en contenedores, con la realización de mediciones insitu utilizando las métricas de retardo extremo a extremo, probabilidad de pérdida de paquete y tiempo de procesamiento.

Desde el punto de vista de los modelos de movilidad de la mula y la predictibilidad asociada a estos modelos, los trabajos futuros irán en la línea de estudiar con mayor detenimiento los factores que influyen en la predictibilidad de la mula por parte de los sensores desplegados en tierra. Los factores actualmente estudiados en la tesis permiten definir un modelo determinista y uno indeterminista, en el que no se permite conocer el comportamiento de la mula. Esa simplificación no existe habitualmente en la realidad, donde situaciones como los planes de vuelo, líneas aéreas, y vuelos periódicos se aplican con mucha frecuencia. Otras limitaciones como las estaciones de carga o las baterías imponen unas limitaciones a los objetos móviles que actúan de mula, esto les obliga a reducir el margen de impredictibilidad.

Una mejor predictibilidad de la mula permite anticiparse al recorrido que efectúa y, en última estancia, aumentar el tiempo de visibilidad de la mula con los nodos interlocutores o cluster head. Este aumento de tiempo de cobertura irá asociado a una mayor duración de las conexiones y a la transmisión de mayor información. Esto también nos obliga a estudiar las características de creación de conexiones a nivel de DTN, y como el tamaño del mensaje o bundle es determinante para una transmisión 
exitosa de la información. Este factor, estudiado en la tesis de (Bedón Monzón, 2016), y artículos relacionados (Bedon, Miguel, Alcarria, Fernández, \& Ruiz, 2016), supondrá un refuerzo para el desarrollo de esta línea de trabajos futuros.

El incremento del tiempo de visibilidad de la mula tiene que venir asociado a contribuciones en el ámbito del re-encaminamiento de paquetes y la cesión/delegación de conexiones, de forma que un conjunto de nodos de una red heterogénea sea capaz de cambiar de nodo interlocutor de manera dinámica, tras el cálculo de la posición futura de la mula. La propuesta del modelo matemático para el cálculo de los tiempos de cobertura, y las estrategias para la elección del nodo interlocutor son aspectos a tratar con detenimiento en el futuro.

El modelo de comunicaciones propuesto en este trabajo de tesis requiere de la coordinación de los diferentes actores que encontramos en el escenario propuesto: sensores y actuadores, nodos interlocutores o cluster-head, elementos móviles recogedores de información o mulas y otras redes conectadas a Internet y a entornos cloud de ejecución de servicios. Aunque la colaboración entre estas entidades se ha presentado en el trabajo de tesis desde el punto de vista de la gestión de servicios (identificando los procesos de delegación, matchmaking, descubrimiento y autoconfiguración), es necesario dotar a los modelos propuestos de otro punto de vista, el de la calidad de servicio percibida por el usuario final que ejecuta este servicio. Por tanto, como trabajo futuro se propone investigar en la elección de parámetros para la cuantificación de la calidad de servicio en este tipo de entornos colaborativos y disruptivos. Los resultados de esta investigación contribuirán a la extensión del modelo de servicio propuesto en la sección 5.2.

En el ámbito de la función de matchmaking o armonización, el algoritmo propuesto compara sintácticamente las condiciones básicas con respecto a los atributos y las restricciones impuestas por la capacidad. Como mejora a esta función, se propone tener en cuenta otras factores, como realizar una comparación de términos definidos en taxonomías $\mathrm{u}$ ontologías en el proceso de matchmaking, mejorando la interoperabilidad, y en el caso de ontologías, permitiendo un acople semántico. Con posibilidades de realizar inferencias para aportar una mayor complejidad el proceso y mayor versatilidad para sensores, actuadores y redes heterogéneas.

Por último, se propone el despliegue de mejoras en el middleware de comunicaciones implementado en la mula, de forma que la interoperabilidad del sistema se amplíe, haciendo el modelo de comunicaciones compatible con tecnologías hasta ahora no soportadas, pero que podrían serlo si se consideran mecanismos de despliegue automático de drivers o controladores utilizando arquitecturas modulares como por ejemplo OSGi (Ramon Alcarria, Robles, Dominguez, \& González-Miranda, 2012). 


\section{Publicaciones}

En el transcurso del desarrollo de este trabajo de investigación, el autor ha realizado un conjunto de publicaciones directamente relacionadas con las contribuciones, y otras indirectamente vinculadas, pero apoyan las ideas y soluciones propuestas.

Publicaciones directamente relacionadas con los objetivos de la tesis:

- Alcarria, R., Robles, T., Dominguez, A. M. \& Cedeno, E (2014). Resolving coordination challenges in distributed mobile service executions. In International Journal of Web and Grid Services (IJWGS) JCR (2012): 1.615 Q1.

- Alcarria, R., Robles, T., Dominguez, A. M. \& Cedeno, E (2012). Resolving Coordination Challenges in Cooperative Mobile Services. In Innovative Mobile and Internet Services in Ubiquitous Computing (IMIS), 2012 Sixth International Conference on, pages 823-828.

- Morales, A., Valladares, T. R., Alcarria, R. \& Cedeño, E. (2013). On the Support of Scientific Workflows over Pub/Sub Brokers. Sensors, 13(8), 10954-10980. JCR (2012): 1.953 Q1

- Morales, A., Alcarria, R., Valladares, T. R. \& Herrera, E. C. (2012). Improving Cooperativity in a Workflow Coordination Model over a Pub/Sub Network. Lecture Notes in Computer Science. Ubiquitous Computing and Ambient Intelligence, , 216-223

- Morales, A., Alcarria, R., Valladares, T. R. \& Herrera, E. C. (2013). An Extended Topic-Based Pub/Sub Broker for Cooperative Mobile Services. The 27th IEEE International Conference on. Advanced Information Networking and Applications.

- Cedeño, E. H., Valladares, T. R., Alcarria, R. \& Morales, A. (2013). On the Characterization of Collaborative Mobile Services for the Internet of Things. The Seventh International Conference on Innovative Mobile and Internet Services in Ubiquitous Computing (IMIS-2013)

- Alcarria, R., Valladares, T. R., Morales, A. \& Herrera, E. C. (2013). A Variability Model for Template Personalization in Mobile Prosumer Environments. The 
27th IEEE International Conference on. Advanced Information Networking and Applications.

- Morales, A., Alcarria, R., Valladares, T. R. \& Herrera, E. C. (2013). E-Flow: A communication system for user notification in dynamic evacuation scenarios. International Conference on Ubiquitous Computing \& Ambient Intelligence

\section{International peer reviewed Journals with ISBN/ISSN}

- Morales, A., Valladares, T. R., Alcarria, R. \& Herrera, E. C. (2013). A Hot topic based Distribution and Notification of Events in PubSub Mobile Brokers. Network Protocols and Algorithms

Otras publicaciones del autor, indirectamente relacionadas con las tesis:

International peer reviewed conferences with proceedings:

- Morales, A., Valladares, T. R., Alcarria, R. \& Herrera, E. C (2014). A Reference Mobile System for Extending Content in Dynamic Domestic Environments. In IEEE International Conference on Consumer Electronics (accepted for publication)

- Dominguez, A. M., Robles, T., Alcarria, R. \& Cedeño, E (2013). A Rendezvous Mobile Broker for Pub/Sub Networks. Lecture Notes of the Institute for Computer Sciences, Social Informatics and Telecommunications Engineering. Green Communication and Networking. Springer

- Dominguez, A. M., Robles, T., Alcarria, R. \& Cedeño, E (2012). Mixed RealityAware Service Architecture for Mobile Environments. In Innovative Mobile and Internet Services in Ubiquitous Computing (IMIS), 2012 Sixth International Conference on, pages 817-822. 


\section{Referencias}

3GPP TR 23.888. (2012, September). Technical Specification Group Services and System Aspects. Retrieved from http://www.qtc.jp/3GPP/Specs/23888-b00.pdf

Abdelhaq, E. J., \& Houria, H. (2008). On actuators number in distributed systems. Sensors and Actuators A: Physical, 147(1), 273- 278.

Aguilera, U., Almeida, A., Orduña, P., López-de-Ipiña, D., \& de las Heras, R. (2010). Continuous service execution in mobile prosumer environments (pp. 229-238). Valencia, Spain.

Akyildiz, I. F., \& Kasimoglu, I. H. (2004). Wireless sensor and actor networks: research challenges. Ad Hoc Networks, 2(4), 351-367. https://doi.org/10.1016/j.adhoc.2004.04.003

Alcarria Garrido, R. P. (2013, November). Contribution to flexible service coordination in mobile prosumer environments (phd). E.T.S.I. Telecomunicación (UPM). Retrieved from http://oa.upm.es/21983/

Alcarria, R., Martín, D., Robles, T., \& Manso, M. Á. (2017). A SPQR-Tree Decomposition Technique for Efficient Cloud Inter-Domain Execution. Journal of Internet Technology, 18(1), 113-124.

Alcarria, R., Martín, D., Robles, T., \& Sánchez-Picot, Á. (2016). Enabling Efficient Service Distribution Using Process Model Transformations. Int. J. Data Warehous. Min., 12(1), 119. https://doi.org/10.4018/IJDWM.2016010101

Alcarria, R., Robles, T., Dominguez, A. M., \& González-Miranda, S. (2012). Flexible Service Composition Based on Bundle Communication in OSGi. TIIS, 6(1), 116-130. https://doi.org/10.3837/tiis.2012.01.007 
Alcarria, R., Robles, T., Morales, A., López-de-Ipiña, D., \& Aguilera, U. (2012). Enabling Flexible and Continuous Capability Invocation in Mobile Prosumer Environments. Sensors, 12(7), 8930-8954. https://doi.org/10.3390/s120708930

Al-Yasiri, A., \& Sunley, A. (2007). Data aggregation in wireless sensor networks using the SOAP protocol. Journal of Physics: Conference Series, 76(1), 012039. https://doi.org/10.1088/1742$6596 / 76 / 1 / 012039$

Arumaithurai, M., Blefari-Melazzi, N., Tagami, A., Seedorf, J., \& Ramakrishnan, K. (2016). Using ICN in disaster scenarios. Retrieved February 16, 2017, from https://tools.ietf.org/html/draft-irtf-icnrg-disaster-00\#section-2

Asim, M., Mokhtar, H., \& Merabti, M. (2008). A Fault Management Architecture For Wireless Sensor Network (pp. 779-785). Presented at the International Conference on Wireless Communications and Mobile Computing (IWCMC). https://doi.org/10.1109/IWCMC.2008.135

Avilés-López, E., \& García-Macías, J. A. (2009). TinySOA: a service-oriented architecture for wireless sensor networks. Service Oriented Computing and Applications, 3(2), 99-108. https://doi.org/10.1007/s11761-009-0043-x

Bedon, H., Miguel, C., Alcarria, R., Fernández, Á., \& Ruiz, F. J. (2016). Message Fragmentation Assessment in DTN Nanosatellite-based Sensor Networks. Ad Hoc Netw., 44(C), 76-89. https://doi.org/10.1016/j.adhoc.2016.02.015

Bedón Monzón, H. M. (2016). Contribution to the improvement of communication services in nanosatellite constellations using delay and disruption tolerant networking based architectures (phd). E.T.S.I. Telecomunicación (UPM). Retrieved from http://oa.upm.es/39650/

Blum, N., Müller, J., Schreiner, F., \& Magedanz, T. (2013). Telecom Applications, APIs and Service Platforms. In E. Bertin, N. Crespi, \& T. Magedanz (Eds.), Evolution of Telecommunication Services (pp. 25-46). Springer Berlin Heidelberg. Retrieved from http://link.springer.com/chapter/10.1007/978-3-642-41569-2_2 
Bordel, B., Rivera, D. S. d, \& Alcarria, R. (2016). Plug-and-Play Transducers in Cyber-Physical Systems for Device-Driven Applications. In 2016 10th International Conference on Innovative Mobile and Internet Services in Ubiquitous Computing (IMIS) (pp. 316-321). https://doi.org/10.1109/IMIS.2016.68

Bordel Sanchez, B., Alcarria Garrido, R., Sánchez-de-Rivera, D., \& Sánchez-Picot, Á. (2016, December). Enhancing Process Control in Industry 4.0 Scenarios using Cyber-Physical Systems. Journal of Wireless Mobile Networks, Ubiquitous Computing, and Dependable Applications, 7(4), 41-64.

Bordel Sánchez, B., Alcarria, R., Martín, D., \& Robles, T. (2015). TF4SM: A Framework for Developing Traceability Solutions in Small Manufacturing Companies. Sensors, 15(11), 29478-29510. https://doi.org/10.3390/s151129478

Burrell, J., Brooke, T., \& Beckwith, R. (2004). Vineyard computing: sensor networks in agricultural production. IEEE Pervasive Computing, 3(1), 38-45. https://doi.org/10.1109/MPRV.2004.1269130

Callaway, E., Gorday, P., Hester, L., Gutierrez, J. A., Naeve, M., Heile, B., \& Bahl, V. (2002). Home networking with IEEE 802.15.4: a developing standard for low-rate wireless personal area networks. IEEE Communications Magazine, 40(8), 70-77. https://doi.org/10.1109/MCOM.2002.1024418

Cheng, J. C. P., Law, K. H., Bjornsson, H., Jones, A., \& Sriram, R. (2010). A service oriented framework for construction supply chain integration. Automation in Construction, 19(2), 245-260. https://doi.org/10.1016/j.autcon.2009.10.003

Chin, A., \& Kontogiannis, K. (2004). m-Roam: a service invocation and roaming framework for pervasive computing. In 18th International Conference on Advanced Information Networking and Applications, 2004. AINA 2004. (Vol. 1, pp. $385-392$ Vol.1). https://doi.org/10.1109/AINA.2004.1283941 
Colitti, W., Steenhaut, K., De Caro, N., Buta, B., \& Dobrota, V. (2011). Evaluation of constrained application protocol for wireless sensor networks. In 2011 18th IEEE Workshop on Local Metropolitan Area Networks $\quad$ (LANMAN) $\quad$ (pp. $1-6)$. https://doi.org/10.1109/LANMAN.2011.6076934

Connected Devices - Ericsson. (2012, December 10). [OpenArticle]. Retrieved September 17, 2015, from http://www.ericsson.com/openarticle/mwc-connected-devices_1686565587_c

Costa, P., Coulson, G., Gold, R., Lad, M., Mascolo, C., Mottola, L., ... Zachariadis, S. (2007). The RUNES middleware for networked embedded systems and its application in a disaster management scenario. Presented at the In Proc. of the 5 th Int. Conf. on Pervasive $\begin{array}{lll}\text { Communications } & \text { (PERCOM. } & \text { Retrieved }\end{array}$ http://citeseerx.ist.psu.edu/viewdoc/versions?doi=10.1.1.102.6828

Costa, P., Mottola, L., Murphy, A. L., \& Picco, G. P. (2007). Programming Wireless Sensor Networks with the TeenyLime Middleware. In Proceedings of the ACM/IFIP/USENIX 2007 International Conference on Middleware (pp. 429-449). New York, NY, USA: Springer-Verlag New York, Inc. Retrieved http://dl.acm.org/citation.cfm?id=1516124.1516153

Coulson, G., Blair, G., Grace, P., Taiani, F., Joolia, A., Lee, K., ... Sivaharan, T. (2008). A Generic Component Model for Building Systems Software. ACM Trans. Comput. Syst., 26(1), 1:11:42. https://doi.org/10.1145/1328671.1328672

Curino, C., Giani, M., Giorgetta, M., Giusti, A., Murphy, A. L., \& Picco, G. P. (2005). Mobile Data Collection in Sensor Networks: The TinyLime Middleware. Pervasive Mob. Comput., 1(4), 446-469. https://doi.org/10.1016/j.pmcj.2005.08.003

Darbee, P. (2013). INSTEON: The Details. Retrieved from http://cache.insteon.com/documentation/insteon_details.pdf 
Davies, E., Tyson, G., Ohlman, B., Pentikousis, K., Eum, S., Corujo, D., ... Boggia, G. (2015). Information-Centric Networking: Baseline Scenarios. Retrieved February 16, 2017, from https://tools.ietf.org/html/rfc7476\#section-2.7

Demirkan, H., Kauffman, R. J., Vayghan, J. A., Fill, H.-G., Karagiannis, D., \& Maglio, P. P. (2008). Service-oriented Technology and Management: Perspectives on Research and Practice for the Coming Decade. Electron. Commer. Rec. Appl., 7(4), 356-376. https://doi.org/10.1016/j.elerap.2008.07.002

Dun-fan, Y., Liang-liang, M., \& Wang, W. (2009). Design and Implementation of Wireless Sensor Network Gateway Based on Environmental Monitoring. In International Conference on Environmental Science and Information Application Technology, 2009. ESIAT 2009 (Vol. 2, pp. 289-292). https://doi.org/10.1109/ESIAT.2009.194

Elvesæter, B., Berre, A.-J., \& Sadovykh, A. (2011). Specifying Services using the Service Oriented Architecture Modeling Language (SoaML) - A Baseline for Specification of Cloud-based Services - Semantic Scholar. Presented at the CLOSER. Retrieved from /paper/Specifying-Services-using-the-Service-Oriented-Elvesæter-

Berre/2e10418ba858cb1f4827fb51e6e582d3cdf5072f

European Telecommunications Standards Institute. (2013). TS 102690 - V1.2.1 - Machine-toMachine communications (M2M); Functional architecture. Retrieved from http://archive.org/details/etsi_ts_102_690_v01.02.01

Farrell, S., Ramadas, M., \& Burleigh, S. (2008, September). Licklider Transmission Protocol $\begin{array}{llll}\text { Motivation. } & \text { Retrieved } & \text { February } & \text { 2017, from }\end{array}$ https://tools.ietf.org/html/rfc5325\#section-3.1

Fok, C.-L., Roman, G.-C., \& Lu, C. (2009). Agilla: A Mobile Agent Middleware for Self-adaptive Wireless Sensor Networks. ACM Trans. Auton. Adapt. Syst., 4(3), 16:1-16:26. https://doi.org/10.1145/1552297.1552299 
Fok, C.-L., Roman, G.-C., \& Lu, C. (2012). Servilla: A Flexible Service Provisioning Middleware for Heterogeneous Sensor Networks. Sci. Comput. Program., 77(6), 663-684. https://doi.org/10.1016/j.scico.2010.11.006

Franco, R. D., Bas, Á. O., \& Esteban, F. L. (2009). Modeling extended manufacturing processes with service-oriented entities. Service Business, 3(1), 31-50. https://doi.org/10.1007/s11628-008-0056-0

Gámez, N., \& Fuentes, L. (2011). FamiWare: A Family of Event-based Middleware for Ambient Intelligence. Personal Ubiquitous Comput., 15(4), 329-339. https://doi.org/10.1007/s00779010-0354-0

Gartmann, R., Holtkamp, B., Weissenberg, N., \& Li, G. (2005). Service roaming in mobile applications. In 2005 IEEE International Conference on Services Computing (SCC'05) Vol-1 (Vol. 1, pp. 112-118 vol.1). https://doi.org/10.1109/SCC.2005.83

Gartner Symposium/ITxpo. (2014). Retrieved September 17, 2015, from http://www.gartner.com/newsroom/id/2905717

Gelernter, D. (1985). Generative Communication in Linda. ACM Trans. Program. Lang. Syst., 7(1), 80-112. https://doi.org/10.1145/2363.2433

Horváth, K. A., \& Telek, M. (2015). Task Delegation in a Peer-to-Peer Volunteer Computing Platform. In Analytical and Stochastic Modelling Techniques and Applications (pp. 115-129). Springer, Cham. https://doi.org/10.1007/978-3-319-18579-8_9

Hughes, D., Thoelen, K., Maerien, J., Matthys, N., Garcia, del C., Javier, P., .. Joosen, W. (2012). LooCI: the Loosely-coupled Component Infrastructure (pp. 236-243). Presented at the 2012 11TH IEEE INTERNATIONAL SYMPOSIUM ON NETWORK COMPUTING $\begin{array}{llll}\text { AND } & \text { APPLICATIONS } & \text { (NCA). } & \text { Retrieved }\end{array}$ https://lirias.kuleuven.be/handle/123456789/351623

Ibrahim, N. (2009). Orthogonal Classification of Middleware Technologies (pp. 46-51). Presented at the Third International Conference on Mobile Ubiquitous Computing, 
Systems, Services and Technologies, 2009. UBICOMM '09. https://doi.org/10.1109/UBICOMM.2009.24

Jara, A. J., Zamora, M. A., \& Skarmeta, A. F. G. (2009). HWSN6: Hospital Wireless Sensor Networks Based on 6LoWPAN Technology: Mobility and Fault Tolerance Management. In International Conference on Computational Science and Engineering, 2009. CSE '09 (Vol. 2, pp. 879-884). https://doi.org/10.1109/CSE.2009.126

Jaradat, W., Dearle, A., \& Barker, A. (2014). Workflow Partitioning and Deployment on the Cloud Using Orchestra. In 2014 IEEE/ACM 7th International Conference on Utility and Cloud Computing (pp. 251-260). https://doi.org/10.1109/UCC.2014.34

Jiang, X., Dawson-Haggerty, S., Dutta, P., \& Culler, D. (2009). Design and Implementation of a High-fidelity AC Metering Network. In Proceedings of the 2009 International Conference on Information Processing in Sensor Networks (pp. 253-264). Washington, DC, USA: IEEE Computer Society. Retrieved from http://dl.acm.org/citation.cfm?id=1602165.1602189

Juang, P., Oki, H., Wang, Y., Martonosi, M., Peh, L. S., \& Rubenstein, D. (2002). Energy-efficient Computing for Wildlife Tracking: Design Tradeoffs and Early Experiences with ZebraNet. In Proceedings of the 10th International Conference on Architectural Support for Programming Languages and Operating Systems (pp. 96-107). New York, NY, USA: ACM. https://doi.org/10.1145/605397.605408

Kansal, A., Nath, S., Liu, J., \& Zhao, F. (2007). SenseWeb: An Infrastructure for Shared Sensing. IEEE MultiMedia, 14(4), 8-13. https://doi.org/10.1109/MMUL.2007.82

Kephart, J. O., \& Chess, D. M. (2003). The vision of autonomic computing. Computer, 36(1), 4150. https://doi.org/10.1109/MC.2003.1160055

Kosanović, M. R., \& Stojčev, M. K. (2011, February 9). CONNECTING WIRELESS SENSOR NETWORKS TO INTERNET. FACTA UNIVERSITATIS, 9(2), 169 - 182. 
Kouche, A. E. (2012). Towards a wireless sensor network platform for the Internet of Things: Sprouts WSN platform. In 2012 IEEE International Conference on Communications (ICC) (pp. 632-636). https://doi.org/10.1109/ICC.2012.6364196

Kovatsch, M., Duquennoy, S., \& Dunkels, A. (2011). A Low-Power CoAP for Contiki. In 2011 IEEE 8th International Conference on Mobile Adhoc and Sensor Systems (MASS) (pp. 855860). https://doi.org/10.1109/MASS.2011.100

Kritikos, K., \& Plexousakis, D. (2014). Novel Optimal and Scalable Nonfunctional Service Matchmaking Techniques. IEEE Transactions on Services Computing, 7(4), 614-627. https://doi.org/10.1109/TSC.2013.11

Kushwaha, M., Amundson, I., Koutsoukos, X., Neema, S., \& Sztipanovits, J. (2007). OASiS: A Programming Framework for Service-Oriented Sensor Networks. In 2nd International Conference on Communication Systems Software and Middleware, 2007. COMSWARE 2007 (pp. 1-8). https://doi.org/10.1109/COMSWA.2007.382431

Li, B., \& Yu, J. (2011). Research and Application on the Smart Home Based on Component Technologies and Internet of Things. Procedia Engineering, 15, 2087-2092. https://doi.org/10.1016/j.proeng.2011.08.390

Lindgren, A., Mascolo, C., Lonergan, M., \& McConnell, B. (2008). Seal-2-Seal: A delay-tolerant protocol for contact logging in wildlife monitoring sensor networks. In 5th IEEE International Conference on Mobile Ad Hoc and Sensor Systems, 2008. MASS 2008 (pp. 321327). https://doi.org/10.1109/MAHSS.2008.4660064

Mainetti, L., Patrono, L., \& Vilei, A. (2011). Evolution of wireless sensor networks towards the Internet of Things: A survey. In 2011 19th International Conference on Software, Telecommunications and Computer Networks (SoftCOM) (pp. 1-6).

Marinho, M. A. M., de Freitas, E. P., Lustosa da Costa, J. P. C., de Almeida, A. L. F., \& de Sousa, J. R.T. (2013). Using cooperative MIMO techniques and UAV relay networks to support connectivity in sparse Wireless Sensor Networks. In 2013 International Conference on 
Computing, Management and Telecommunications (ComManTel) (pp. 49-54). https://doi.org/10.1109/ComManTel.2013.6482364

Mayer, K., \& Fritsche, W. (2006). IP-enabled Wireless Sensor Networks and Their Integration into the Internet. In Proceedings of the First International Conference on Integrated Internet Ad Hoc and Sensor Networks. New York, NY, USA: ACM. https://doi.org/10.1145/1142680.1142687

McDonald, P., Geraghty, D., Humphreys, I., Farrell, S., \& Cahill, V. (2007). Sensor Network with Delay Tolerance (SeNDT). In Proceedings of 16th International Conference on Computer Communications and Networks, 2007. ICCCN 2007 (pp. 1333-1338). https://doi.org/10.1109/ICCCN.2007.4318006

Mikhaylov, K., \& Tervonen, J. (2013). Data Collection from Isolated Clusters in Wireless Sensor Networks Using Mobile Ferries. In 2013 27th International Conference on Advanced Information Networking and Applications Workshops (WAINA) (pp. 903-909). https://doi.org/10.1109/WAINA.2013.87

Mitton, N., Papavassiliou, S., Puliafito, A., \& Trivedi, K. S. (2012). Combining Cloud and sensors in a smart city environment. EURASIP Journal on Wireless Communications and Networking, 2012(1), 247. https://doi.org/10.1186/1687-1499-2012-247

Moeller, R., \& Sleman, A. (2008). Wireless networking services for implementation of ambient intelligence at home. In 2008 7th International Caribbean Conference on Devices, Circuits and Systems (pp. 1-5). https://doi.org/10.1109/ICCDCS.2008.4542655

Morais, Y., \& Elias, G. (2010). Integrating Communication Paradigms in a Mobile Middleware Product Line (pp. 255-261). Washington, DC, USA: IEEE Computer Society. https://doi.org/10.1109/ICN.2010.48

MQTT. (2014). Retrieved from http://mqtt.org/

MQTT Version 3.1.1 (OASIS Standard). (2014, October 29). Retrieved from http://docs.oasisopen.org/mqtt/mqtt/v3.1.1/os/mqtt-v3.1.1-os.pdf 
Nanda, M. G., Chandra, S., \& Sarkar, V. (2004). Decentralizing execution of composite web services (pp. 170-187). ACM Press.

OASIS. (2004, October 19). UDDI Version 3.0.2. Retrieved April 18, 2017, from http://www.uddi.org/pubs/uddi_v3.htm

Paczesny, T., Tajmajer, T., Domaszewicz, J., \& Pruszkowski, A. (2012). ProxyMotes: Linux-based TinyOS Platform for Non-TinyOS Sensors and Actuators. In 2012 IEEE 10th International Symposium on Parallel and Distributed Processing with Applications (ISPA) (pp. 255-261). https://doi.org/10.1109/ISPA.2012.41

Perumal, B., Rajasekaran M., P., \& Ramalingam, H. M. (2012). WSN Integrated Cloud for Automated Telemedicine (atm) based e-healthcare applications (pp. 166- 170). Presented at the International Conference on Bioinformatics and Biomedical Technology, Singapore.

Pignaton de Freitas, E., Heimfarth, T., Netto, I. F., Lino, C. E., Pereira, C. E., Ferreira, A. M., ... Larsson, T. (2010). UAV relay network to support WSN connectivity. In 2010 International Congress on Ultra Modern Telecommunications and Control Systems and Workshops (ICUMT) (pp. 309-314). https://doi.org/10.1109/ICUMT.2010.5676621

Piyare, R., Park, S., Maeng, S. Y., Park, S. H., Oh, S. C., Choi, S. G., .. Lee, S. R. (2013). Integrating Wireless Sensor Network into Cloud services for real-time data collection. In 2013 International Conference on ICT Convergence (ICTC) (pp. 752-756). https://doi.org/10.1109/ICTC.2013.6675470

Pohl, K., Böckle, G., \& Linden, F. J. van der. (2005). Software Product Line Engineering: Foundations, Principles and Techniques. Secaucus, NJ, USA: Springer-Verlag New York, Inc.

Polyvyanyy, A., Vanhatalo, J., \& Völzer, H. (2011). Simplified Computation and Generalization of the Refined Process Structure Tree. In M. Bravetti \& T. Bultan (Eds.), Web Services and 
Formal Methods (pp. 25-41). Springer Berlin Heidelberg. Retrieved from http://link.springer.com/chapter/10.1007/978-3-642-19589-1_2

Priyantha, B., Kansal, A., Goraczko, M., \& Zhao, F. (2008). Tiny Web Services for Sensor Device Interoperability. In 2008 International Conference on Information Processing in Sensor Networks (ipsn 2008) (pp. 567-568). https://doi.org/10.1109/IPSN.2008.33

Qiu, P., Heo, U., \& Choi, J. (2009). The web-sensor gateway architecture for Zigbee. In IEEE 13th International Symposium on Consumer Electronics, 2009. ISCE '09 (pp. 661-664). https://doi.org/10.1109/ISCE.2009.5156996

Ramanuja, V., Zhenyun, Z., \& Raghupathy, S. (2006). Hazard avoidance in wireless sensor and actor networks. Computer Communications, 29(13-14), 2578-2598. https://doi.org/10.1016/j.comcom.2006.02.012

Rebollo, O., Mellado, D., \& Fernandez-Medina, E. (2015). ISGcloud: a Security Governance Framework for Cloud Computing. The Computer Journal, 58(10), 2233-2254. https://doi.org/10.1093/comjnl/bxu141

Ruiz-Ibarra, E., \& Villasenor-Gonzalez, L. (2008). Cooperation mechanism taxonomy for wireless sensor and actor networks (pp. 62-73). Presented at the International Federation for Information Processing.

Selavo, L., Wood, A., Cao, Q., Sookoor, T., Liu, H., Srinivasan, A., ... Porter, J. (2007). LUSTER: Wireless Sensor Network for Environmental Research. In Proceedings of the 5th International Conference on Embedded Networked Sensor Systems (pp. 103-116). New York, NY, USA: ACM. https://doi.org/10.1145/1322263.1322274

$\begin{array}{lllll}\text { Semantic Sensor } \quad \text { Network } & \text { Ontology. } & \text { (n.d.). } & \text { Retrieved }\end{array}$ https://www.w3.org/2005/Incubator/ssn/ssnx/ssn

Shelby, Z., \& Bormann, C. (2009). Wiley: 6LoWPAN: The Wireless Embedded Internet. $\begin{array}{llll}\text { Retrieved September } & \text { 17, 2015, from }\end{array}$ http://eu.wiley.com/WileyCDA/WileyTitle/productCd-0470747994.html 
Shelby, Z., Hartke, K., \& Bormann, C. (2012). The Constrained Application Protocol (CoAP). Retrieved September 18, 2015, from https://tools.ietf.org/html/draft-ietf-core-coap-13

Sheltami, T., Al-Roubaiey, A., Mahmoud, A., \& Shakshuki, E. (2015). A publish/subscribe middleware cost in wireless sensor networks: A review and case study. In 2015 IEEE 28th Canadian Conference on Electrical and Computer Engineering (CCECE) (pp. 1356-1363). https://doi.org/10.1109/CCECE.2015.7129476

Shenker, S., Partridge, C., \& Guerin, R. (1997). Specification of Guaranteed Quality of Service. ACM.

Steenkamp, L., Kaplan, S., \& Wilkinson, R. H. (2009). Wireless sensor network gateway. In AFRICON, 2009. AFRICON '09. https://doi.org/10.1109/AFRCON.2009.5308112

Strassner, J., Meer, S. van der, Foghlu, M. O., Leon, M. P. de, \& Donnelly, W. (2009). Autonomic Orchestration of Future Networks to Realize Prosumer Services. In 2009 International Conference on Future Networks (pp. 152-156). https://doi.org/10.1109/ICFN.2009.61

Taherkordi, A., Loiret, F., Abdolrazaghi, A., Rouvoy, R., Le-Trung, Q., \& Eliassen, F. (2010). Programming Sensor Networks Using REMORA Component Model. In Proceedings of the 6th IEEE International Conference on Distributed Computing in Sensor Systems (pp. 4562). Berlin, Heidelberg: Springer-Verlag. https://doi.org/10.1007/978-3-642-13651-1_4

Taherkordi, A., Loiret, F., Rouvoy, R., \& Eliassen, F. (2013). Optimizing Sensor Network Reprogramming via in Situ Reconfigurable Components. ACM Trans. Sen. Netw., 9(2), 14:1-14:33. https://doi.org/10.1145/2422966.2422971

Tarjan, R. E., \& Valdes, J. (1980). Prime subprogram parsing of a program (pp. 95-105). New York, NY, USA: ACM. https://doi.org/10.1145/567446.567456

Ting-hui, H., Sang, T., \& Yi-min, Y. (2010). An architecture for integrating wireless sensor networks into IP network. In 2010 International Conference on Intelligent Computing and Integrated Systems (ICISS) (pp. 910-912). https://doi.org/10.1109/ICISS.2010.5657038 
Tohidi, H. (2011). Modelling of business services in service oriented enterprises. Procedia Computer Science, 3, 1147-1156. https://doi.org/10.1016/j.procs.2010.12.186

Vanhatalo, J., Völzer, H., \& Koehler, J. (2008). The Refined Process Structure Tree. In M. Dumas, M. Reichert, \& M.-C. Shan (Eds.), Business Process Management (pp. 100-115). Springer Berlin Heidelberg. Retrieved from http://link.springer.com/chapter/10.1007/978-3-54085758-7_10

Vanhatalo, J., Völzer, H., \& Leymann, F. (2007). Faster and More Focused Control-Flow Analysis for Business Process Models Through SESE Decomposition. In B. J. Krämer, K.-J. Lin, \& P. Narasimhan (Eds.), Service-Oriented Computing - ICSOC 2007 (pp. 43-55). $\begin{array}{llll}\text { Springer } & \text { Berlin } & \text { Heidelberg. } & \text { Retrieved }\end{array}$ http://link.springer.com/chapter/10.1007/978-3-540-74974-5_4

Vasseur, J.-P., \& Dunkels, A. (2010). Interconnecting Smart Objects with IP. The Next Internet. Elsevier Inc. Retrieved from http://www.elsevier.com/books/interconnecting-smartobjects-with-ip/vasseur/978-0-12-375165-2\#

Villareal Padilla, J. E., \& Jung Ho Kim, J.-O. L. (2013). A 3GPP IP Multimedia Subsystem-Based System Architecture for a M2M Horizontal Service Platform (Vol. Vol. 4 Issue 8, p. p299). Presented at the Journal of Next Generation Information Technology. Retrieved from http://connection.ebscohost.com/c/articles/98924660/3gpp-ip-multimediasubsystem-based-system-architecture-m2m-horizontal-service-platform

Wang, F., \& Liu, J. (2011). Networked Wireless Sensor Data Collection: Issues, Challenges, and Approaches. IEEE Communications Surveys Tutorials, 13(4), 673-687. https://doi.org/10.1109/SURV.2011.060710.00066

Weißenberg, N., Gartmann, R., \& Voisard, A. (2006). An Ontology-Based Approach to Personalized Situation-Aware Mobile Service Supply. GeoInformatica, 10(1), 55-90. https:/doi.org/10.1007/s10707-005-4886-9 
Wolff, A., Michaelis, S., Schmutzler, J., \& Wietfeld, C. (2007). Network-centric Middleware for Service Oriented Architectures across Heterogeneous Embedded Systems. In 2007 Eleventh International IEEE EDOC Conference Workshop (pp. 105-108). https://doi.org/10.1109/EDOCW.2007.20

Wood, D., \& Robson, D. (2012). Message broker technology for flexible signalling control. Presented at the ASPECT 2012.

Yu, Q., Wang, R., Zhao, K., Li, W., Sun, X., Hu, J., \& Ji, X. (2016). Modeling RTT for DTN Protocol Over Asymmetric Cislunar Space Channels. IEEE Systems Journal, 10(2), 556567. https://doi.org/10.1109/JSYST.2014.2330422

Zensys A/S. (2007, May). Z-Wave: “Z-Wave Protocol Overview”, Versión 4. Retrieved from http://wiki.ase.tut.fi/courseWiki/images/9/94/SDS10243_2_Z_Wave_Protocol_Overview. pdf

Zhang, T., Ying, S., Cao, S., \& Jia, X. (2006). A Modeling Framework for Service-Oriented Architecture. In 2006 Sixth International Conference on Quality Software (QSIC'06) (pp. 219-226). https://doi.org/10.1109/QSIC.2006.3

ZigBee Alliance, I. (2008, December). ZigBee Smart Energy Profile Specification, Revision 15.

ZigBee Alliance, I. (2010a, January). ZigBee RF4CE Specification, Version 1.01.

ZigBee Alliance, I. (2010b, February 8). ZigBee Home Automation Public Application Profile, Revision 26, Version 1.1. 